\title{
Ionenstrahlinduzierte selbst-organisierte Musterbildung auf einfachen Oberflächen Theorie und Experiment
}

\author{
Dissertation \\ zur Erlangung des Doktorgrades \\ der Mathematisch-Naturwissenschaftlichen Fakultäten \\ Dr.rer.nat. \\ der Georg-August-Universität zu Göttingen \\ im Promotionsprogramm ProPhys \\ der Georg-August University School of Science (GAUSS)
}

vorgelegt von

Omar Bobes

aus Damaskus, Syrien

Göttingen 2018 


\section{Betreuungsausschuss:}

Prof. Dr. Hans Hofsäss, II. Physikalisches Institut

Dr. Kun Zhang, II. Physikalisches Institut

\section{Mitglieder der Prüfungskommission:}

Referent: Prof. Dr. Hans Hofsäss, II. Physikalisches Institut

Korreferentin: Prof. Dr. Astrid Pundt, Institut für Materialphysik

\section{Weitere Mitglieder der Prüfungskommission:}

Prof. Dr. Michael Seibt, IV. Physikalisches Institut

Prof. Dr. Wolfram Kollatschny, Institut für Astrophysik

Prof. Dr. Vasily Moshnyaga, I. Physikalisches Institut

Prof. Dr. Ariane Frey, II. Physikalisches Institut

Tag der mündlichen Prüfung: 15.05.2018 
Im Namen Gottes, des Allerbarmers, des Barmherzigen 
Mein Lehrer und Erzieher, der mich lehrte, erst zu denken, um zu wählen was ich will, und nicht zu wollen und dann meinen Verstand zu zwingen, wie er denken soll

\author{
Der Märtyrer \\ Prof. Dr. M. S. Al-Buti
}

(Scheich der Levante)

Gott möge sich seiner erbarmen und sich seine Werke annehmen 


\section{Abstract}

In the thesis at hand, ripple pattern formation on amorphous carbon and Si surfaces has been investigated at room temperature during low energy $\mathrm{Ne}^{+}$and $\mathrm{Ar}^{+}$ion irradiation as a function of the ion incidence angle. Monte Carlo simulations of the curvature coefficients applied to the Bradley-Harper and Cater-Vishnyakov models, including the recent extensions predict that pattern formation on amorphous carbon thin films should be possible for low energy $\mathrm{Ne}^{+}$ions from $250 \mathrm{eV}$ up to $1500 \mathrm{eV}$. Moreover, simulations are able to explain the absence of pattern formation for low energy $\mathrm{Ne}^{+}$ions on Si. Our experimental results are compared with prediction using current linear theoretical models and applying the crater function formalism, as well as Monte Carlo simulations to calculate curvature coefficients using the SDTrimSP program. Calculations indicate that no patterns should be generated up to $45^{\circ}$ incidence angle if the dynamic behavior of the thickness of the ion irradiated layer is taken into account, while pattern formation most pronounced from $50^{\circ}$ for ion energy between $250 \mathrm{eV}$ and $1500 \mathrm{eV}$, which are in good agreement with our experimental data.

Furthermore ripple pattern formation on amorphous carbon films has been investigated during normal incidence ion beam erosion under simultaneous deposition of different metalic co-deposited surfactant atoms. ta-C films were irradiated using $1 \mathrm{keV} \mathrm{Ar}{ }^{+}$ions under continuous deposition of Ti, W, Mo and Pt surfactants. The co-depotion of small amounts $\mathrm{Ti}, \mathrm{W}$ and $\mathrm{Mo}$ leads to the steady state formation of $\mathrm{TiC}, \mathrm{WC}$ or $\mathrm{MoC}$ nanocomposite surface of few nm thickness. This has a tremendous impact on the evolution of nanoscale surface patterns on ta-C. While the surface keeps always flat under co-deposition of Ptatoms, where there is no possibility for phase separation, ripple patterns are observed under co-deposition of Ti-, W- and Mo-atoms. The results confirm that the phase separation is the major driving force for the pattern formation in the case of irradiation with normal incident beam.

Keywords: ion irradiation, surfactant sputtering, pattern formation. 


\section{Zusammenfassung}

In der vorliegenden Arbeit wurde die Musterbildung auf amorphen Kohlenstoff- und Si-Oberflächen bei Raumtemperatur während der Bestrahlung von niederenergetischen $\mathrm{Ne}^{+}$-und $\mathrm{Ar}^{+}$-Ionen als eine Funktion des Ioneneinfallswinkels untersucht. Monte-CarloSimulationen der Krümmungskoeffizienten, die auf die theoretische Modelle angewendet wurden, einschließlich der neulich eingeführten Erweiterungen, konnten die Musterbildung auf amorphen Kohlenstoff für niederenergetische $\mathrm{Ne}^{+}$-Ionen von $250 \mathrm{eV}$ bis 1500 $\mathrm{eV}$ vorhersagen. Darüber hinaus sind die Simulationen in der Lage, die Abwesenheit von Musterbildung für niederenergetische $\mathrm{Ne}^{+}$-Ionen auf Si zu erklären. Berechnungen zeigen, dass keine Muster bis zu einem Einfallswinkel von $45^{\circ}$ erzeugt werden sollten, wenn das dynamische Dickenabhängigkeit der bestrahlten Schicht berücksichtigt wird. Auf die andere Seite taucht die Musterbildung ab 50 für Ionenenergien zwischen $250 \mathrm{eV}$ und 1500 $\mathrm{eV}$.

Weiterhin wurde in dieser Arbeit die Bildung von Rippelmustern auf amorphen Kohlenstoffschichten bei normalem Ioneneinfall unter gleichzeitiger Kodeposition verschiedener metallischer Fremdatome untersucht. Wir haben ta-C-Schichten mit $1 \mathrm{keV} \mathrm{Ar}^{+}$-Ionen unter kontinuierlicher Deposition von Ti-, W-, Mo- und Pt-Surfactant bestrahlt. Die Kodeposition von kleinen Mengen Ti, W und Mo führt zur Bildung von TiC-, WC- oder MoC-Nanokompositen mit einer Dicke von wenigen nm. Dies hat einen enormen Einfluss auf die Entwicklung von Oberflächenmustern auf ta-C. Während die Oberfläche unter Kodeposition von Pt-Atomen immer flach bleibt, wo es keine Möglichkeit für Phasenseparation gibt, werden Rippel-Muster unter Kodeposition von Ti-, W- und Mo-Atomen beobachtet. Die Ergebnisse bestätigen, dass die Phasenseparation die Hauptantriebskraft für die Musterbildung im Fall einer senkrechten Ionenbestrahlung.

Stichwörter: Ionenbestrahlung, Surfactant Sputtering, Musterbildung 


\section{Inhaltsverzeichnis}

\begin{tabular}{ll}
\hline 1. Einleitung & 1
\end{tabular}

2. Theorie der Musterbildung 5

2.1. Die Sputter-Theorie von Sigmund . . . . . . . . . . . . . . . . . . . . 5

2.2. Die lineare Kontinuumstheorie von Bradley-Harper . . . . . . . . . . . . . 7

2.3. Carter-Vishnyakov Modell . . . . . . . . . . . . . . . . . . . . 10

2.4. Ionenstrahlinduziertes viskoses Fließen . . . . . . . . . . . . . . . . . . . . 13

2.5. Der Kraterfunktionsformalismus . . . . . . . . . . . . . . . . . . . . . . 16

2.6. Erweiterungen der Modelle . . . . . . . . . . . . . . . . . . . . . . . 19

2.6.1. Die erosive Krümmungsabhängigkeit der Kraterfunktion . . . . . . 19

2.6.2. Das erweiterte Carter-Vishnyakov Modell . . . . . . . . . . . . . . . 22

2.6.3. Die Rolle der Ionenimplantation . . . . . . . . . . . . . . . . . . . . 31

2.7. Gemeinsame Betrachtung der Theorien . . . . . . . . . . . . . . . . . 34

2.8. Bestimmung der Krümmungskoeffzienten der Bewegungsgleichung . . . . . 36

2.8.1. Berechnung der Erosionskraterfunktion . . . . . . . . . . . . . . . 39

2.8.2. Berechnung der Redistributionskraterfunktion . . . . . . . . . . . . 40

2.8.3. Berechnung der Dickenabhängigkeit $D_{11} \ldots \ldots$. . . . . . . . . . 41

2.8.4. Berechnung der Implantationskraterfunktion . . . . . . . . . . . . . 41

2.8.5. Bestimmung des Koeffizients für ioneninduziertes viskoses Fließen . 42

2.9. Surfactant Sputtering . . . . . . . . . . . . . . . . . . . . . . . . . 44

\begin{tabular}{lr}
\hline 3. Methoden & 47
\end{tabular}

3.1. Probenpräparation . . . . . . . . . . . . . . . . . . . 47

3.2. Ionenbestrahlung . . . . . . . . . . . . . . . . . . . 47

3.2.1. Die Plasmaquelle . . . . . . . . . . . . . . . . . . . 47

3.2 .2 . Ionenbescheuniger IOSCHKA . . . . . . . . . . . . . . . . 49

3.2.3. Ionenbeschleuniger ADONIS . . . . . . . . . . . . . . . . . . . 50

3.3. Mess- und Analyseverfahren . . . . . . . . . . . . . . . . . . . . . 51

3.3.1. Rasterkraftmikroskop . . . . . . . . . . . . . . . . . . . 51

3.3.2. Rutherford-Rückstreu-Spektrometrie . . . . . . . . . . . . 53 
4. Ionenstrahlinduzierte Musterbildung auf einfachen Oberflächen 57

4.1. Einführung . . . . . . . . . . . . . . . . . . . 57

4.2. Ionenstrahlinduzierte Musterbildung auf a-C Schichten . . . . . . . . . . 57

4.2.1. Bestrahlung mit $\mathrm{Ne}^{+}$-Ionen . . . . . . . . . . . . . . . 57

4.2.2. Simulationsergebnisse . . . . . . . . . . . . . . . . . . . 59

4.2.3. Bestrahlung mit $\mathrm{Ar}^{+}$-Ionen . . . . . . . . . . . . . . 67

4.2.4. Simulationsergebnisse . . . . . . . . . . . . . . . . . 69

4.2.5. Diskussion . . . . . . . . . . . . . . . . . . . 73

4.2.6. Fazit . . . . . . . . . . . . . . . . . . . 74

4.3. Ionenstrahlinduzierte Musterbildung auf Si Schichten . . . . . . . . . . . . 75

4.3.1. Bestrahlung mit Ne-Ionen . . . . . . . . . . . . . . . 75

4.3.2. Simulationsergebnisse . . . . . . . . . . . . . . . . . 75

4.3.3. Diskussion und Fazit . . . . . . . . . . . . . . . . . . . . . . 81

5. Ionenstrahlinduzierte Musterbildung auf a-C mit gleichzeitigen Kodeposition von Fremdatomen $\quad 83$

5.1. Einführung . . . . . . . . . . . . . . . . . 83

$5.2 . \quad$ Ergebnisse . . . . . . . . . . . . . . . . . 86

5.3. Diskussion und Fazit . . . . . . . . . . . . . . . . . . . . . . 90

\begin{tabular}{ll}
\hline 6. Zusammenfassung & 93
\end{tabular}

\begin{tabular}{lr}
\hline 7. Ausbilck & 97
\end{tabular}

\begin{tabular}{lr}
\hline A. Publikationen & 127
\end{tabular}

\begin{tabular}{lr}
\hline B. Danksagung & 129
\end{tabular} 


\title{
Nomenklatur
}

\author{
Abkürzungen
}

\begin{tabular}{ll} 
Abkürzung & Bedeutung \\
\hline SIMS & SekundärIonen-MassenSpektrometrie \\
SMS & Sigmund Model of Sputtering \\
BH & Bradley-Harper \\
CV & Carter-Vishnyakov \\
CFF & Crater Function Formalism \\
MD & Molecular Dynamics \\
MC & Monte-Carlo \\
BCM & Binary Collision Method \\
SRIM & Stopping and Range of Ions in Matter \\
TRIM & Transport of Ions in Matter \\
ZBL & Ziegler-Biersack-Littmark \\
TRIDYN & TRIm simulation code including DYNamic composition \\
KC & Krypton-Carbon \\
SDTrimSP & Static and Dynamic Transport of Ions in Matter Sequential and \\
Pa.dpa & Parallel processing \\
IWS & Pascal displacements per atom \\
IPP & Institut für Werkstoff- und Strahlentechnik \\
IOSCHKA & Institut für PlasmaPhysik \\
ADONIS & IOnen SCHleuder KAmmer \\
AFM & Anlage zur DepOsition von Niederenergetischer Ionen \\
RBS & Substrate \\
DLC & Atomic Force Microscope \\
PSD & Rutherford Backscattering Spectrometry \\
& Diamond Like Carbon \\
Power Spectral Density \\
\end{tabular}


Nomenklatur

\begin{tabular}{ll} 
Abkürzung & Bedeutung \\
\hline Surfactant & Surface active agents \\
SEM & Scanning Electron Microscope \\
TEM & Transmission Electron Microscopy \\
FIB & Focused Ion Beam
\end{tabular}




\section{Einleitung}

Die Erosion von Festkörper-Oberflächen mit niederenergetischen Ionen und bei schrägem Einfallswinkel könnte eine periodische selbst-organisierte Nanostruktur mit potentiellen Anwendungen erzeugen [16, 34, 35]. Neben den üblichen Anwendungen der Erosion zur Herstellung von dünnen Schichten und tiefenaufgelösten Analyse der Zusammensetzung von Oberflächen (SIMS) ${ }^{1}$ sind nanostrukturierte Oberflächen mit abstimmbarer Symmetrie und charakteristischer Längenskala und Periodizität für photonische Anwendungen [52], oberflächenverstärkte Raman-Spektroskopie und nanostrukturierte biokompatible Oberflächen von zunehmender Bedeutung [12, 84, 86, 138]. Außerdem wurden ionenstrahlnanostrukturierte metallische Oberflächen wie z.B. Anordnungen von Nanodrähten hinsichtlich der plasmonischen Funktionalität untersucht [51, 121, 143]. Gerade in den letzten zehn Jahren wurde festgestellt, dass ioneninduzierte Rippelmuster auf ferromagnetischen Dünnschichten eine abstimmbare ausgeprägte magnetische Textur erzeugen [26, 89, 133, 152, 155, 156]. Darüber hinaus wurden Wellen-geordnete Nanostrukturen, die durch niederenergetische Stickstoff-Ionenbestrahlung von Si erzeugt wurden, als periodische Nanomaske untersucht, um die Kanalregion von Feldeffekttransistoren zu dotieren [140].

Seit der Entdeckung von ionenstrahlinduzierten Oberflächenstrukturen von Navez et al. [114, 115] im Jahre 1962, gab es viele Untersuchungen, um dieses Phänomen zu verstehen. Die frühen Stadien der selbst-organisierten Musterbildung beruhen üblicherweise auf lineare theoretische Beschreibungen wie die Erosionstheorie von Bradley-Harper (BH-Theorie) [22] und das Massenredistribution-Modell von Carter-Vishnyakov (CVModell) [28]. Erweiterungen für nichtlineare Terme und Rauschen wurden unter Verwendung der Kuramoto-Sivashinsky-Gleichung eingeführt [10, 112, 126]. Andere theoretische Modelle basieren auf ioneninduziertem Stress [27, 117], viskoses Fließen und chemischen Effekten sowie Phasenseparation durch gleichzeitige Kodeposition von Fremdatomen [32, 68, 111, 131. Ein Ansatz beschreibt Erosion sowie Massentransport auf der Basis von Kraterfunktionen. Die meisten Analysen, die die Kraterfunktion verwenden, beruhen auf Molekulardynamik-Simulationsdaten und sind daher auf Ionen mit sehr nied-

\footnotetext{
${ }^{1}$ SekundärIonen-MassenSepktrometrie.
} 


\section{Einleitung}

riger Energie und einfache elementare Systeme beschränkt [81, 82, 118, 119]. Allerdings fehlt bisher ein umfassendes Verständnis der Musterbildungsprozesse und ein quantitative Vorhersage der Musterbildung ist kaum möglich [72]. Existierende theoretische Modelle können bisher die ionenstrahlinduzierte Musterbildung nur für bestimmte Fälle quantitativ vorhersagen und stimmen oft nicht mit experimentellen Beobachtungen überein. Zum Beispiel ist bisher die Abwesenheit von Rippelmustern bei einer Bestrahlung von Si-Oberflächen mit leichten Ionen wie Ar oder Ne im Energiebereich zwischen 1-10 keV nicht zu erklären.

Kürzlich haben Harrison und Bradley gezeigt, dass die bisher verwendete theoretische Beschreibung der Musterbildung basierend auf dem Kraterfunktionsformalismus unvollständig ist und wichtige Erweiterung erfordert [61]. Ebenso wurden von unserer Gruppe kürzlich Erweiterungen zur Theorie der Musterbildung durch Redistribution und Ionenimplantation eingeführt [70, 75]. Es ist erwähnenswert, dass das Phänomen der Musterbildung ziemlich kompliziert ist, und ein quantitatives Verständnis dieses Phänomens erfordert einen Vergleich von Experiment, Simulation und Theorie.

In der vorliegenden Arbeit wird ionenstrahlinduzierte selbst-organisierte Musterbildung auf einfachen Oberflächen aus experimenteller Sicht behandelt. Die Arbeit präsentiert verschiedene Arten von Experimenten zur Musterbildung auf amorphen Kohlenstoff- und Silizium-Oberflächen mit niederenergetischen Leichtionen. Ziel ist es Gültigkeit und Genauigkeit der vorhandenen theoretischen Modelle zu überprüfen und zu zeigen, dass die Musterbildung auf Silizium und amorphen Kohlenstoff nur mit Kombination der erweiterten linearen Theorien quantitativ vorhersagbar ist. Durch Monte-Carlo-Simulationen der Ion-Festkörper-Wechselwirkung ist es möglich, die für die Berechnung der Bewegungsgleichungen eines Oberflächenprofils notwendigen Krümmungskoeffizienten mit hoher Genauigkeit zu berechnen. Aus diesen Koeffizienten können quantitative Vorhersagen über die Stabilität oder Instabilität einer Oberfläche, Wachstumraten, Wellenlängen sowie die laterale Bewegung der Muster gewonnen worden [18, 65, 72]. Außerdem wurde in dieser Arbeit ein zusätzlicher Beweis für die ioneninduzierte Phasenseparation als initialem Prozess zur selbst-organisierten Musterbildung unter dem Einfluss von metallischen Fremdatomen geliefert.

Die Arbeit gliedert sich in folgende Teile:

\section{Kapitel 1: Einleitung}

Kapitel 2: Einführung in die Theorie der ioneninduzierten Musterbildung einschließlich der kürzlich eingeführten Erweiterungen. In diesem Kapitel wurde auch die Bestimmung 
der Krümmungskoeffizienten der Bewegungsgleichung mit Hilfe des Monte-CarloSimulationsprogramms SDTrimSP erläutert.

Kapitel 3: Vorstellung der in dieser Arbeit verwendeten Methoden für die Bestrahlung und Charakterisierung der Proben.

Kapitel 4: Darstellung der experimentellen Ergebnissen für die Bestrahlung von Silizium-Substraten und amorphen Kohlenstoffschichten mit nieder- und mittelenergetischen $\mathrm{Ne}^{+}$- und $\mathrm{Ar}^{+}$-Ionen.

Kapitel 5: Untersuchung der Bildung von Rippelmustern auf amorphen Kohlenstoffschichten bei normaler Ionenstrahl-Erosion unter gleichzeitiger Deposition verschiedener metallischer Fremdatome.

Kapitel 6: Zusammenfassung der Ergebnisse.

Kapitel 7: Schlussbetrachtung und Ausblick der Arbeit. 



\section{Theorie der Musterbildung}

Durch den Beschuss eines Festkörpers mit energiereichen Ionen werden unterschiedliche Wechselwirkungen zwischen Ionen und Targetatomen hervorgerufen. Die Wechselwirkung kann je nach Energie des Projektils zur Inkorporation von Ionen (Implantation), zu Strahlenschäden und Versetzungen von Substratatomen (engl.: mass redistribution), zur Emission von Atomen (Sputter-Erosion) und bei niedrigen Energien unter 100 eV zur Ionendeposition auf der Oberfläche führen. Die gezielte Ionenbestrahlung von Feskörpern kann zu verschiedenen selbst-organisierten periodischen Mustern führen. Abhängig von Einfallswinkel, Ionensorte und Ionenenergie kommt es unter bestimmten Umständen zu einem Punkt- bzw. Wellenmuster oder die Oberfläche wird im einfachsten Fall geglättet. In diesem Kapitel werden die Grundlagen zur Musterbildungstheorie, vor allem das SputterModell von Sigmund [136, 137] und die darauf basierende Lineare Kontinuumstheorie von Bradley und Harper [22], sowie das CV-Modell von Carter und Vishnyakov [28] und das ionenstrahlinduzierte viskose Fließen von Castro und Cuerno vorgestellt [31 33]. Darüber hinaus werden in diesem Kapitel die für die obengenannten Theorien erforderlichen Erweiterungen eingeführt sowie die Rolle der Ionen-Implantation erklärt. Am Ende des Kapitels wird die ionenstrahlinduzierte Musterbildung durch Kodeposition von Fremdatomen vorgestellt.

\subsection{Die Sputter-Theorie von Sigmund}

Die meisten ionenstrahlinduzierten Musterbildungstheorien basieren auf dam Sputtermodell von Sigmund, kurz SMS (Sigmund Model of Sputtering) [136]. SMS ist die erste anerkannte Theorie zur Beschreibung und Berechnung des Sputteryields (Zahl der emittierten Atome pro einfallendem Ion). Sigmund führte in seiner Arbeit einen Aufrauungsmechanismus ein, der abhängig von der Oberflächenkrümmung ist und mit der Annahme, dass der Sputteryield propotional zu der an der Targetoberfläche deponierten Energie ist. Der Sputterprozess erfolgt nach Sigmund in der folgende Weise: Durch den Beschuss einer Festkörperoberfläche mit energiereichen Ionen werden Atome, die sich innerhalb der wenigen Atomlagen unterhalb der Targetoberfläche befinden aufgrund der elastischen Stöße 
eine Impulskomponenten in Richtung der Oberflächennormale übertragen und die Potentialschwelle an der Oberfläche (Bindungsenergie) überwunden. Sigmund geht in seinem Modell von einem isotropen, in alle Raumrichtungen unendlich ausgedehnten Medium, aus, sodass sich sein Modell nur auf amorphe und polykristalline Target anwenden lässt. Mit dieser Annahme hat Sigmund einen mathematischen Ausdruck für den Sputteryield $Y$ hergeleitet [136]

$$
Y=\Lambda F_{D}(E, \theta)
$$

Der Faktor $\Lambda$ ist eine Materialkonstante, die von der Oberflächenbindungsenergie und der atomaren Dichte des Festkörpers abhängt. Die Energiedichtefunktion $F_{D}(E, \theta)$ stellt die Energie dar, die an der Oberfläche deponiert wird. Die Berechnung von $F_{D}(E, \theta)$ erfolgt oftmals analytisch mit der Nährung, dass die deponierte Energie gaußförmig verteilt ist und meistens stimmt der damit berechnete Sputteryield für viele Ion-TargetKombinationen gut mit experimentellen Ergebnisse überein [136].

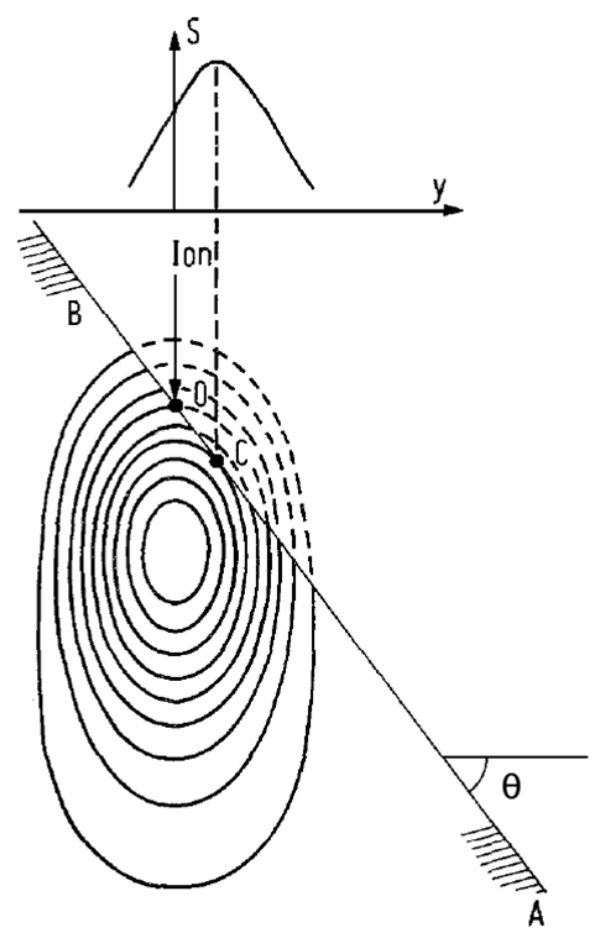

$\boldsymbol{A} \boldsymbol{b} \boldsymbol{b}$. 2.1.: Die gaußförmige Energieverteilung des einfallenden Ions. Die Isolinien verbinden Punkte gleicher Energiedeposition. Das Maximum der Funktion $F_{D}(E, \theta)$ liegt in der Mitte (aus [137]).

Eine anschaulische Darstellung des Sputterprozesses nach Sigmund [136] lässt sich durch 
ellipsoidische gaußförmige Energieverteilung zeigen. In Abbildung (2.1) ist ein Konturplot der Funktion $F_{D}(E, \theta)$ dargestellt. Die Strecke AB ist die Targetoberfläche. Das einfallende Ion dringt mit dem Winkel $\theta$ im Punkt 0 in das Target ein und verliert beim Durchlaufen seine kinetische Energie. Die Auswertung der Funktion $F_{D}(E, \theta)$ an der Oberfläche zeigt, dass das Maximum der lokalen Sputterrate nicht bei Punkt 0, sondern bei Punkt C liegt. Die Abhängigkeit des Sputteryields eines Targets vom Einfallswinkel $\theta$ lautet nach dem Sigmund-Modell

$$
Y(\theta)=Y(0) \cdot(\cos )^{-n}
$$

wobei $n$ vom Massenverhältniss $\left(\frac{M_{t}}{M_{i}}\right)$ des Targetatoms und des Ions abhängt, für $\left(\frac{M_{t}}{M_{i}}\right) \leq 3$ ist $n \approx 5 / 3 . Y(0)$ ist der Sputteryield bei senkrechten Ioneneinfall. Diese Abhängigkeit gilt für Einfallswinkel bis zu ungefähr $75^{\circ}$, jedoch fällt der Sputteryield für Einfallswinkel $\theta \geq$ $75^{\circ}$ extrem ab (bis zu null für $\theta=90^{\circ}$ ). Der Grund dafür ist, dass bei streifendem IonenEinfall ein großer Teil der einfallenden Ionen an der Oberfläche reflektiert wird, sodass die Targetatome nicht genug kinetische Energie haben, um die Oberflächenbindungsenergie zu überwinden und das Target zu verlassen. Diese Abweichung führte M. Bradley und H. Hofsäss zu einer Modifikation des Sigmund-Modells, in der sie die scheinbare Ablenkung der Ionen zur Oberfläche beim Eintritt in den Festkörper berücksichtigt haben [73, 97].

\subsection{Die lineare Kontinuumstheorie von Bradley-Harper}

Basierend auf Sigmunds Theorie haben M. Bradley und J. M. E. Harper ein Modell für die Erklärung der Rippelmorphologie entwickelt (BH-Theorie) [22]. Sie zeigten, dass die Variation des Sputteryields, der von der lokalen Oberflächenkrümmung abhängig ist, zur Bildung von Wellenmustern führt. Die Abhängigkeit des Sputteryields mit der Oberflächenkrümmung kann man anhand der Abbildung (2.2) erklären. Bei senkrechtem Ioneneinfall (Abbildung 2.2 a) ist die gesamte Energie, die an der stelle O deponiert wird, höher als die deponierte Energie an der Stelle $\mathrm{O}^{\prime}$. Der Grund dafür ist, dass die gesamte Energie, die an der Stelle O bzw. O' deponiert wird, nicht nur von Ionen, die in O bzw. O' auftreten, stammt, sondern auch auf die Punkte A, B bzw. A', B' zurückgeht. Das Zentrum der Energieverteilung von den Punkten A, B zu O weist jeweils einen kleineren Abstand als das von A', B' zu O' auf. Da jedoch eine erhöhte Energiedeposition auch gleichzeitig einen erhöhten Sputteryield bedeutet, ist bei senkrechtem Ioneneinfall die Erosion in einem Tal höher als auf einem Hügel. Für große Einfallswinkel (relativ zur Oberflächennormalen) 

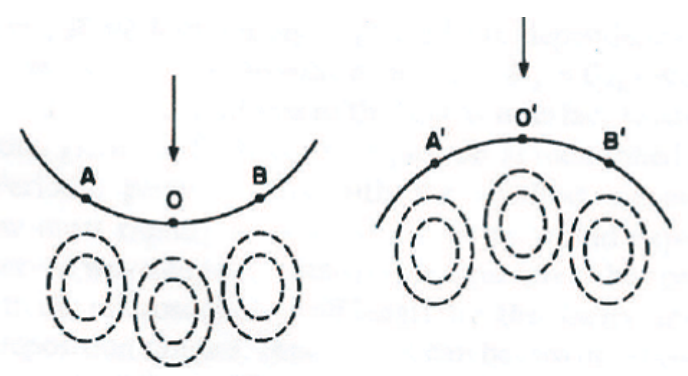

a)
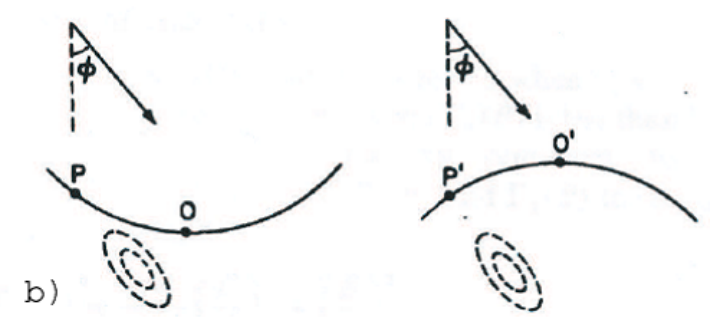

$\boldsymbol{A} \boldsymbol{b} \boldsymbol{b}$. 2.2.: Krümmungsabhängige Energieverteilung von Ionen a) bei senkrechtem Einfall und b) bei streifendem Einfall [22].

ist der Energiebeitrag vom Punkt $\mathrm{P}$ zu O zwar immer noch größer als der Energiebeitrag vom Punkt $\mathrm{P}^{\prime}$ zu $\mathrm{O}^{\prime}$, jedoch ist die Anzahl der Ionen, die am Punkt $\mathrm{P}^{\prime}$ auftritt größer als am Punkt P. Dadurch ist die Sputterrate am Punkt $\mathrm{O}^{\prime}$ größer als die am Punkt O (Abbildung 2.2 b).

Für die Entstehung der Rippelmuster werden zwei entgegengesetzte Mechanismen verantwortlich gemacht, zum einen die Erosion (aufrauender Prozess) und zum anderen die Oberflächendiffusion (glättender Prozess). Um den Sputteryield an einem Punkt O auf die Oberfläche zu ermitteln, wird neben einem globalen Koordinatensystem, dessen z-Achse othogonal zur unerodierten, glatten Oberfläche steht, ein lokales Koordinatensystem $\left(x^{\prime}\right.$, $y^{\prime}, z^{\prime}$ ) definiert, dessen $z^{\prime}$-Achse in Ionenstrahlrichtung zeigt (siehe Abbildung 2.3).

Der Sputteryield an einem bestimmten Punkt auf die Oberfläche ist proportional zur Energie, die durch die Ionen deponiert wird. Die Energieverteilung wird für $\vec{r}=\overrightarrow{0}$ durch

$$
F_{\overrightarrow{0}}\left(x^{\prime}, y^{\prime}, z^{\prime}\right)=\frac{\epsilon}{(2 \pi)^{2 / 3} \sigma \mu^{2}} \cdot \exp \left[-\frac{\left(z^{\prime}-a\right)^{2}}{2 \sigma^{2}}-\frac{x^{\prime 2}+y^{\prime 2}}{2 \mu^{2}}\right]
$$

beschrieben [22, 136]. Dabei ist $\epsilon$ die gesamte deponierte Energie des Ions, $a$ ist die mittlere Tiefe der Energiedeposition und $\sigma$ und $\mu$ sind die Breiten der Verteilung in Strahlrichtung und senkrecht dazu. Ausgehend von Sigmunds Sputtermodell haben Bradley und Harper die Oberfläche als zeitabhängige Höhenfunktion $h(x, y . t)$ beschrieben. Die Koordinaten $x$ und $y$ beschreiben die zweidimensionale Oberfläche, wobei die $x$-Achse parallel zum projizierten Ionenstrahl steht und die $y$-Achse senkrecht dazu. Die zeitliche Entwicklung 


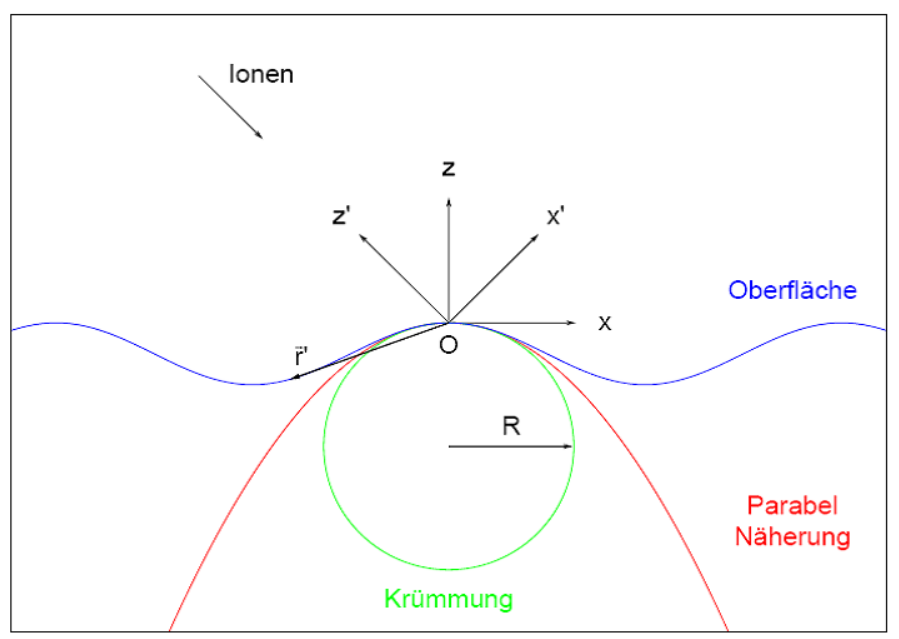

Abb. 2.3.: Koordinatensystem zur Berechnung des Sputteryields nach BH-Theorie. R ist der Radius für die Krümmung im Punkt O. Die Achsen $x$ und $x^{\prime}$ liegen in der Einfallsebene [25].

der Oberflächenhöhe lautet nach Ref. [22]:

$$
\frac{d h}{d t}=-v(\theta)=-v_{0}(\theta)+\frac{\partial v_{0}(\theta)}{\partial \theta} \frac{\partial h}{\partial x}+S_{x} \frac{\partial^{2} h}{\partial x^{2}}+S_{y} \frac{\partial^{2} h}{\partial y^{2}}-B \nabla^{4} h
$$

Der erste Term $v(\theta)$ ist die Oberflächengeschwindigkeit, $v_{0}(\theta)$ ist die Erosionsrate der ungekrümmten Oberfläche nach Sigmund, $\theta$ der Einfallswinkel. $S_{x}$ und $S_{y}$ sind die Krümmungskoeffizienten in $x$ - bzw. $y$-Richtung, die von Ionenenergie, Einfallswinkel und Materialeigenschaften abhängig sind. Der letzte Term $-B \nabla^{4} h=B\left(\frac{\partial^{4} h}{\partial x^{4}}+\frac{\partial^{4} h}{\partial y^{4}}\right)$ beschreibt die thermisch bedingte Oberlächendiffusion mit

$$
B=\frac{B_{s} \gamma \Omega^{2} N}{k_{B} T} \cdot \exp \left(-\frac{\Delta E}{k_{B} T}\right),
$$

wobei $D_{s}$ die Oberflächendiffusionskonstante, $\gamma$ die freie Oberflächenenergie, $\Omega$ das Atomvolumen, $\Delta E$ die Aktivierungsenergie für Oberflächendiffusion, $k_{B}$ die Boltzmann-Konstante und $T$ die Oberflächentemperatur ist [63, 110]. Aufgrund der Linearität von Gl.(2.4) ist es ausreichend, periodische Lösungen mit dem Ansatz

$$
h(x, y, t)=-v_{0} \cdot t+h_{0} e^{\left(i\left(q_{x} \cdot x+q_{y} \cdot y-\omega t\right)\right)} \cdot e^{R t}
$$

zu betrachten. Die Lösung der Differentialgleichung führt zu folgenden Korrelationen [22]

$$
v_{0}^{\prime}(\theta)=-\frac{\partial \omega}{\partial q_{x}} \text { und }
$$




$$
R=-S_{x} q_{x}^{2}-S_{y} q_{y}^{2}-B\left(q_{x}^{2}+q_{y}^{2}\right)^{2}
$$

$v_{0}^{\prime}(\theta)$ beschreibt die laterale Geschwindigkeit des Rippelmusters. $q_{x}, q_{y}$ sind die Wellenvektoren in $x$ - und $y$-Richtung und $R$ ist die Wachstumsrate der Amplitude $h_{0} . S_{x}$ für Einfallswinkel, die nicht allzu groß relativ zur Oberflächennormalen sind, negativ, d.h. eine instabile Oberflächenhöhe in $x$-Richtung. In diesem Fall bilden sich Wellenmuster mit Wellenvektor parallel zur Ionenstrahlrichtung. Für große Einfallswinkel ist $S_{y}$ negativ, was zu einer instabilen Oberflächenhöhe in $y$-Richtung führt und in diesem Fall bilden sich Wellenmuster mit Wellenvektor senkrecht zur Ionenstrahlrichtung. Im Gegensatz zu den Krümmungskoeffizienten ist der Oberflächendiffusionsterm $B$ immer positiv und führt zur Stabilisierung der Oberfläche. Durch die Krümmungskoeffizienten $S_{x, y}$ werden die Orientierung und Wellenlänge der Rippel bestimmt durch

$$
\lambda_{i}=\frac{2 \pi}{q_{i}}=\left(\frac{2 B}{\left|S_{i}\right|}\right)^{1 / 2},
$$

wobei $i$ auf die Richtung ( $x$ oder $y$ ) verweist, in der der zugehörige Koeffizient $S_{i}$ am größten ist.

\subsection{Carter-Vishnyakov Modell}

G. Carter und V. Vishnyakov gehen in ihrem Modell [28] genau wie Bradley und Harper von der Sigmund-Sputtertheorie aus, berücksichtigen in ihrer Näehrung aber den Massentransport, der durch die Kollisionskaskade der rückstoßenden Atome entsteht und welcher einen wichtigen Beitrag für die Entstehung von Musterbildung liefert. Die Kollisionskaskaden, die durch die einfallenden Ionen induziert werden, führen zu Hunderten von versetzten Tragetatomen entlang der Ionentrajektorien, was man letztendlich als Massentransport betrachtet. Der Massentransport pro Einfallsion kann als mittlere Differenz zwischen den End- und Startpositionen der rückgestoßenen Atome, multipliziert mit der Anzahl der entstehenden Leerstellen pro Einfallsion, beschrieben werden. Bei senkrechtem (relativ zur Targetoberfläche) Ioneneinfall ist der mittlere Massentransport pro Ion gegeben durch:

$$
\delta=N_{D} \cdot\left(d_{R}-d_{V}\right)
$$

Hier ist $N_{D}$ die Anzahl der permanent verschobenen rückgestoßenen Atome pro Ion, $d_{R}$ und $d_{V}$ sind jeweils die mittleren Tiefenverteilungen der rückgestoßenen Atome und Leer- 
stellen (siehe Abbildung 2.4. Carter und Vishnyakov untersuchten in Ref. [1] die Bestrah-

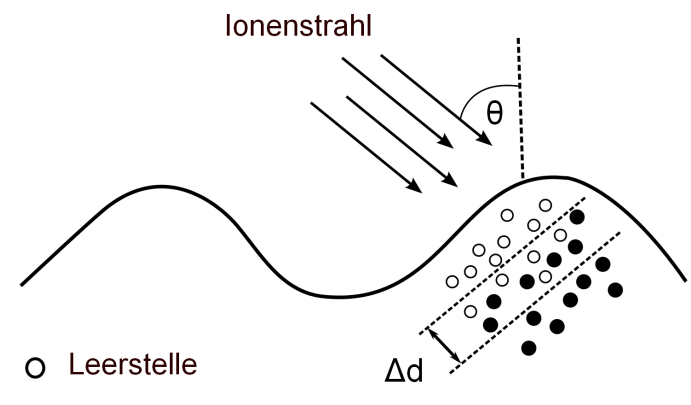

- Recoil-Atom

Abb. 2.4.: Schematische Dartellung des Massentransportes durch Ionenbeschuss. $\theta$ ist der Einfallswinkel bezüglich Oberflächennormale. $\Delta=d_{R}-d_{V}$.

lung von Siliziumoberflächen mit relativ hochenergetischen Xenon-Ionen zwischen 10 - 40 $\mathrm{keV}$ und unter Einfallswinkeln zwischen $0^{\circ}$ bis $45^{\circ}$ relativ zur Oberflächennormalen. Für Einfallswinkel von $>45^{\circ}$ wurde ein deutliches Rippelmuster beobachtet. Für Einfallswinkel $<45^{\circ}$ tauchen jedoch keine Rippel auf. Die Abwesenheit von Rippelmustern bei Einfallswinkeln kleiner als $45^{\circ}$ erklärten Carter und Vishnyakov durch die Einführung eines Atomflusses $F(s) \sqrt{1}$ in $s$-Richtung parallel zur lokalen Oberfläche (Abbildung 2.5):

$$
\begin{gathered}
F(s)=J \cos (\tilde{\theta}) \delta_{\|}, \\
\delta_{\|}=\delta f(E) \sin (\tilde{\theta}) .
\end{gathered}
$$

Hierbei ist $J$ der Ionenfluss und $\tilde{\theta}$ der lokale Einfallswinkel. Die lokale Oberflächennormale zeigt eine kleine Steigung $\frac{\partial h}{\partial x}$ und deswegen wird sie um einem Winkel $\varphi$ (mit $\left.\varphi=\tan \left(\frac{\partial h}{\partial x}\right) \approx \frac{\partial h}{\partial x}\right)$ relativ zur mittleren Oberflächennormalen geneigt. Der lokale Einfallswinkel der Ionen ist somit durch

$$
\tilde{\theta}=\theta-\varphi=\theta-\frac{\partial h}{\partial x}
$$

gegeben. Die Kosinusfunktion in Gleichung (2.11) beschreibt die Veränderung des Ionenflusses mit der Erhöhung des Einfallswinkels. Die Sinusfuktion in Gleichung (2.12) beschreibt die Projektion der Verschiebung von rückgestoßenen Atomen entlang der Oberfläche. $\delta$ ist durch Gleichung (2.10) gegeben. $f(E)$ ist im CV-Modell [28] durch die erzeugten

\footnotetext{
${ }^{1}$ Der Atomfluss $F(s)$ ist hier mit ungewöhnlicher Demension gegben und zwar als $\left[\frac{A t}{m \cdot s}\right] \operatorname{statt}\left[\frac{A t}{m^{2} \cdot s}\right]$. Der Grund dafür ist, dass im CV-Modell die Dicke der bestrahlten Schicht $\frac{1}{D(\theta)}$ ignoriert oder als konstant und unabhängig vom Ioneneinfallswinkel angenommen wurde, wie es später im Abschnitt (2.6.2) ausgeführt wird.
} 

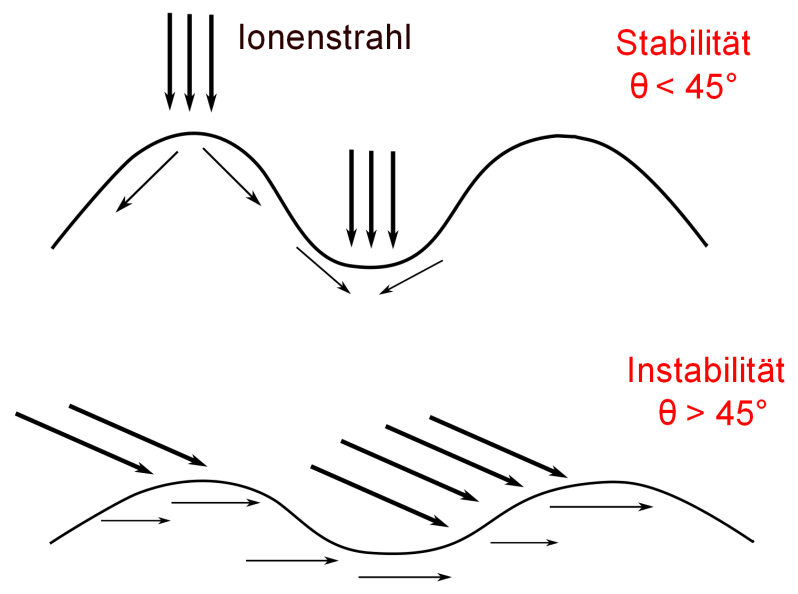

Abb. 2.5.: Massentransport führt für Einfallswinkel $0<\theta<45^{\circ}$ zu eine Oberflächenstabilität. Für Einfallswinkel $\theta>45^{\circ}$ führt die Veränderung des Massentranspotes entlang der Richtung $s$ (parallel zur lokalen Oberfläche) zu einer Oberflächeninstabilität.

rückgestoßenen Atome pro einfallendem Ion festgelegt:

$$
f(E)=\frac{k(E)}{2 E_{D}}
$$

wobei $k(E)$ ein Bruchteil der deponierten Ionenenergie und $E_{D}$ die Verlagerungsenergie (engl.: displacement energy) ist. Mit Berücksichtigung des Redistributionsterms erhalten Carter und Vishnyakov für die zeitliche Entwicklung der Oberflächenhöhe in $x$-Richtung eine ähnliche Gleichung wie Gl. (2.4):

$$
\frac{\partial h}{\partial t}=v_{0}(\theta)+\frac{\partial v_{0}(\theta)}{\partial \theta} \frac{\partial h}{\partial x}+S_{x} \frac{\partial^{2} h}{\partial x^{2}}+\frac{1}{N} \frac{\partial F(s)}{\partial x}-B \nabla^{4} h+\eta(x, t) .
$$

Der totale Atomfluss in $x$-Richtung parallel zur lokalen Oberfläche ist durch

$$
\frac{1}{N} \frac{\partial}{\partial x} F(x)=\frac{1}{N} f(E) \delta \cos (2 \theta) \frac{\partial^{2} h}{\partial x^{2}}
$$

gegeben. Das CV-Modell ist allgemein nur für kleine Einfallswinkel gültig. Die $\sin (\theta)$ Nährung in Gleichung (2.12) versagt für große Einfallswinkel, da die Zahl der versetzten Atome $N_{D}$ pro Ion in Gleichung 2.10 mit einem Einfallswinkel von annährend $90^{\circ}$ stark abnimmt. Darüber hinaus verschwindet bei großen Einfallswinkeln ein Teil des Volumens 
der Kollisionskaskaden über die Oberfläche, sodass die $\cos (\theta)$-Nährung in Gleichung 2.12 nicht mehr gilt. Daher ist eine zuverlässige Ermittlung des Parameters $\delta$ nur durch MonteCarlo oder molekulardynamische Simulationen möglich, wie es später im Abschnitt (2.8) erklärt wird.

\subsection{Ionenstrahlinduziertes viskoses Fließen}

Ein hydrodynamisches Modell, das von M. Castro und R. Cuerno eingeführt wurde [3133], bezeichnet das ionenstrahlinduzierte viskose Fließen als den dominierenden Effekt bei der Musterbildung. Die Beschreibung des viskosen Fließens geht auf die Arbeit von Orchard[122] zurück. In dieser Arbeit wird näherungsweise eine einfache Beziehung für die viskose Strömung in sehr dünnen Schichten mit $q \cdot D<<1$ abgeleitet, wobei $q$ und $D$ jeweils der Wellenvektor und die Schichtdicke sind. Dieser Effekt kann sowohl zu Oberflächenglättung als auch Aufrauung und somit zur Entstehung von Rippelmustern führen. Castro und Cuerno beschreiben die ioninduzierte amorphe Oberflächenschicht als hochviskose Flüssigkeit mit der Dicke $D$, die in der Größenordnung der Ionenreichweite liegt. Bei der Bestrahlung amorpher bzw. amorphisierbarer Systeme mit niederenergetischen Ionen führt die beim Stoß übertragene Energie zu einer Versetzung der Targetatome von ihren Gitterplätzen, wenn diese Energie die Verlagerungsenergie übersteigt. Die rückgestoßenen Atome können entsprechend ihrer Energie weitere Gitteratome versetzen und so kommt es zu einer ganzen Kollisionskaskade, wie in Abbildung (2.6) gezeigt.

Der Gesamteffekt dieses Prozesses ist dreifach:

- Amorphisierung des Materials

- Verspannung der amorphen Schicht

- Massentransport

Die amorphe Schicht bildet sich schnell, ihre Dicke und andere mechanische Eingenschaften werden nach einer Fluenz $>10^{14}$ Ionen $/ \mathrm{cm}^{2}$ stationär und die Dichte des Targets in der amorphen Phase bleibt konstant ${ }^{2}$ In der Sprache der Strömungsmechanik gehen Castro und Cuerno in ihrem Modell von einer amorphen hochviskosen Schicht aus, die inkompressibel mit $(\nabla \cdot \vec{V}=0)$ fließt, wobei $\vec{V}$ das Geschwindigkeitsfeld der flüssigen

\footnotetext{
${ }^{2} \mathrm{Im}$ Castro-Cuerno-Modell und auch in der Literatur wird oft angenommen, dass die Dicke der bestrahlten Schicht konstant ist, obwohl die Dicke von der Ionenenergie und dem Einfallswinkel abhängig ist, wie es später im Abschnitt (2.6.2) gezeigt wird.
} 


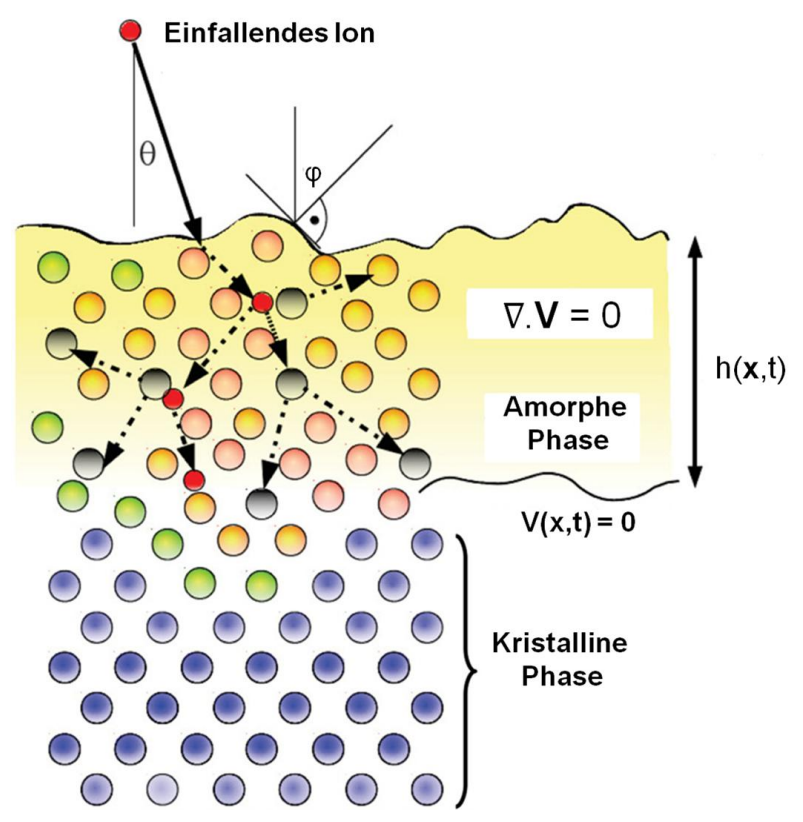

Abb. 2.6.: Beim Eindringen eines energiereichen Ionen in die Oberfläche mit Einfallswinkel $\theta$ stößt es mit Targetatomen und erzeugt eine Kollisionskaskade, die letztendlich durch viele einfallende Ionen zu einer Amorphisierung des oberflächennahen Bereichs führt. Die amorphe Schicht fließt wie eine hochviskose Flüssigkeit. $\varphi$ ist die lokale Steigung des Oberflächenprofils $h(x, t), V$ ist die Fließgeschwindigkeit [33].

Schicht ist. Für ein hochviskoses Medium gilt die Navier-Stokes-Gleichung

$$
\rho \frac{d \mathbf{V}}{d t}=\nabla \cdot \mathbf{T}_{i, j}+\vec{b}=0
$$

$\rho$ ist die Dichte des Targets in der amorphen Phase, $\mathbf{T}_{i, j}$ ist die Spannungstensor und $\vec{b}$ ist die Volumenkraft. $\nabla \cdot \mathbf{T}$ beschreibt die viskose Kraft, die langsam als Scherwirkung für inkompressible Strömung wirkt. An der amorph-kristallinen Grenzfläche gilt die Haftbedingung (engl.: No-Slip) mit $V(x, t)=0$. Die Ionenstrahl wird als Volumenkraft $\vec{b}$ im Target eingebaut und enthält die im Target durch die Massen-Redistribution entstandene Restspannung [31. Der Mechanismus von ionenstrahlinduziertem viskosem Fließen ist ähnlich dem einer Flüssigkeit, die eine geneigte Ebene mit Neigungswinkel $\theta$ herunterfließt. In dieser Analogie ersetzt die externe Volumenkraft $\vec{b}$ die Gravitationskraft. Jedoch ist im Gegensatz zur Gravitationskraft die Volumenkraft nicht konstant, sondern an den Stellen größer, die den Ionenstrahlen direkt ausgesetzt sind (siehe Abbildung 2.7). Die Differenz zwischen der Kraft auf den beiden Seiten der Welle lässt sie für Einfallswinkel $\theta>\theta_{c}$ lateral schrumpfen und so wächst die Wellenamplitude durch die Inkompressibi- 
lität der amorphen Oberfläche. Die charakteristische Musterwellenlänge ist gegeben durch

$$
\lambda_{c}=2 \pi \sqrt{\frac{2 \sigma}{-f_{E} \cos (2 \theta)}} .
$$

Hier $\sigma$ ist die Oberflächenenergie. Für den Koeffizienten $f_{E}$ gilt: $f_{E}=\frac{\varsigma}{d}$, wobei $\varsigma$ die ioneninduzierte Restspannung und $d$ die Dicke der amorphen Schicht sind [31].
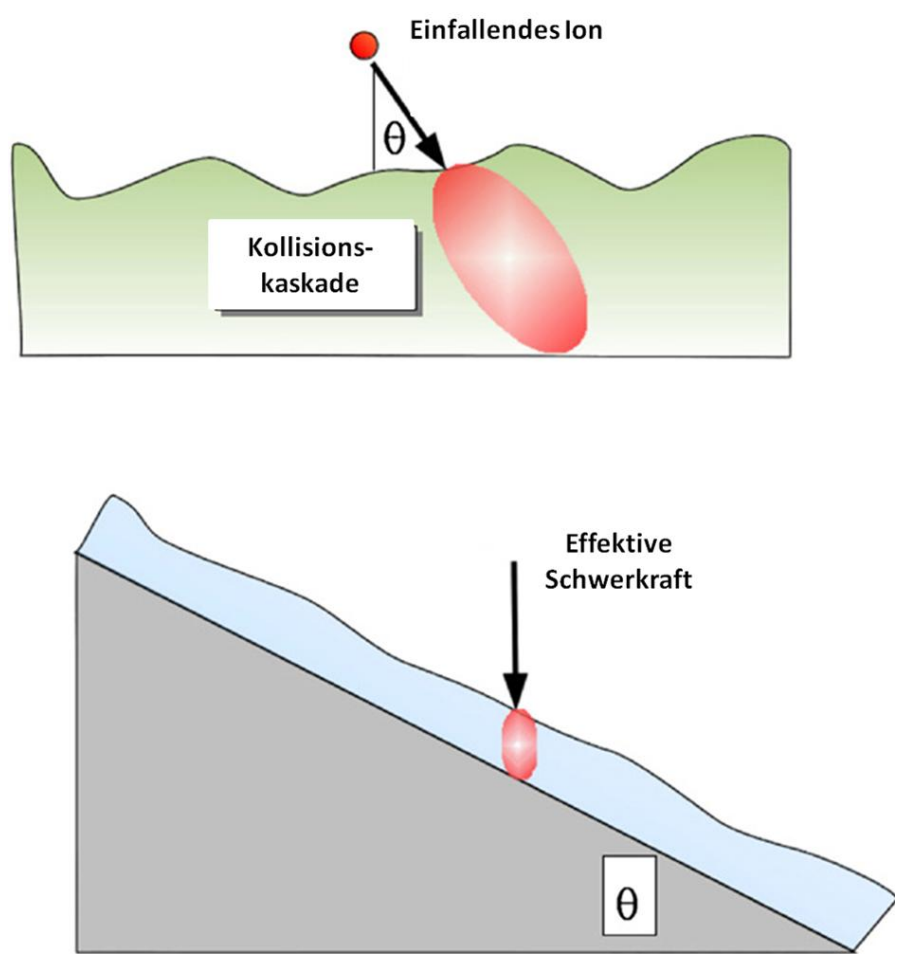

$\boldsymbol{A} \boldsymbol{b} \boldsymbol{b}$. 2.7.: Analogie zwischen Ionenstrahlsputtern und Flüssigkeitsströmung. Das Ionenstrahlsputternsystem kann als eine Flüssigkeit, die eine geneigte Ebene mit Neigungswinkel $\theta$ herunterfließt, gesehen werden. Die externe Volumenkraft $\vec{b}$ ersetzt hier die Gravitationskraft [32]. 


\subsection{Der Kraterfunktionsformalismus}

Der Kraterfunktionsformalismus CFF (Crater Function Formalism) ist eine attraktive Annährung zur Beschreibung sowohl der linearen als auch der nicht linearen Musterbildungstheorien [78, 79, 81, 82, 118, 119]. Der CFF nutzt das durchschnittliche Ergebnis vieler Ioneneinschläge, um eine Greensche Funktion (Kraterfunktion) zu erstellen, die dann verwendet wird, um die Reaktion der bestrahlten Oberfläche zu bestimmen. Die Kraterfunktion beschreibt die durchschnittliche Entwicklung der Oberfläche nach einem einzelnen Ioneneinschlag. Frühere theoretische Studien haben gezeigt, dass eine kleine Veränderung der Kraterform zu erheblichen Veränderungen im Verhalten des makroskopischen Systems führen kann. Laut Norris et al. führt das Bestrahlen von Festkörpern mit niederenergetischen Ionen $\left(10^{2}-10^{4} \mathrm{eV}\right)$ meistens zur schnellen Amorphisierung der Oberflächenschicht $\left(\sim 10^{-3} \mathrm{~s}\right)$. Über längere Zeit $(\sim 100 \mathrm{~s})$ verursacht der Massentransport einen allmählichen Entspannungseffekt [118, 119]. Die Oberflächengeschwindigkeit am Punkt r (siehe Gl. 2.4) kann als

$$
v(\mathbf{r})=v_{P}(\mathbf{r})+v_{G}(\mathbf{r})
$$

geschrieben werden. $v_{G}(\mathbf{r})$ repräsentiert hier das ionenstrahlinduzierte viskose Fließen (Langzeit-Regime) und ist durch

$$
v_{G}(\mathbf{r})=-\frac{\gamma d^{3}}{3 \eta} \cdot \nabla_{\mathbf{S}}^{2} K
$$

gegeben, wobei $\gamma$ die Oberflächenenergie ist, $d$ ist die Dicke der viskosen Schicht und $\eta$ ist die Schicht-Viskosität. $\nabla_{\mathbf{S}}^{2} K$ ist der Laplace-Operator der mittleren Krümmung [12]. Die Oberflächengeschwendigkeit $v_{P}(\mathbf{r})$ im Kurzzeit-Regime kann als die Integration der Kraterfunktion über mehrere Ioneneinschläge im Punkt $\mathbf{r}^{\prime}$ (unter der Berücksichtigung der Ioneneinschläge in der Nähe von $\left.\mathbf{r}^{\prime}\right)$ multipliziert mit dem Ionenfluss $J\left(\mathbf{r}^{\prime}\right)$ geschrieben werden

$$
v_{P}(\mathbf{r})=\int J\left(\mathbf{r}^{\prime}\right) F\left(\mathbf{r}-\mathbf{r}^{\prime}, \theta\right) d \mathbf{r}^{\prime}
$$

Mit der Tatsache, dass die Längenskala der Krater viel kleiner als die resultierende Musterbildung und die Ionenflussverteilung ist, sodass die Oberfläche sehr langsam variiert, haben Norris et al. mit Hilfe einer Multi-Skalen-Analyse die kleine Längenskala der Krater mit der charakteristischen Skala der Musterbildung verbunden und $v_{P}(\mathbf{r})$ in folgender 
Weise approximiert [118]:

$$
v_{P}(\mathbf{r})=\left(J M^{(0)}\right)+\varepsilon \nabla_{\mathbf{S}} \cdot\left(J M^{(1)}\right)+\frac{1}{2} \varepsilon^{2} \nabla_{\mathbf{S}} \cdot \nabla_{\mathbf{S}} \cdot\left(J M^{(2)}\right)+\cdots
$$

Hier ist $\nabla_{\mathbf{S}}$ die Oberflächen-Divergenz, $\varepsilon$ ist das Verhältnis der durchschnittlichen Ioneneindringtiefe zu einer mit der makroskopischen Oberflächenentspannung verbundenen Längenskala mit

$$
\varepsilon=\left(\frac{v_{0} \eta}{\gamma}\right)^{1 / 3}
$$

wobei $v_{0}$ die Erosionsgeschwindigkeit ist. $M^{(n)}$ sind die Momente der Kraterfuktion $F(\mathbf{r}, \theta)$ und sind in der Form geschrieben

$$
\begin{array}{ll}
M^{(0)} & =\int F(\mathbf{r}, \theta) d \mathbf{r} \\
M_{i}^{(1)} & =\int F(\mathbf{r}, \theta) \xi_{i} d \mathbf{r}, \quad \text { mit } \quad i=\{1,2\} ; \xi_{1}=x ; \xi_{2}=y \\
M_{i j}^{(2)} & =\int F(\mathbf{r}, \theta) \xi_{i} \xi_{j} d \mathbf{r}, \quad \text { mit } \quad i, j=\{1,2\} ; \xi_{1}=x ; \xi_{2}=y
\end{array}
$$

Mit der Annahme, dass die Höhenvariation der Oberfläche viel größer als die Länge der Kollisionskaskade ist, lässt sich die zeitliche Entwicklung der Oberflächenhöhe unter Berücksichtigung ausschließlich linearer Terme in folgender Form darstellen:

$$
\frac{d h}{d t}=C_{0}(\theta)+C_{1}(\theta) \frac{\partial h}{\partial x}+C_{11}(\theta) \frac{\partial^{2} h}{\partial x^{2}}+C_{22}(\theta) \frac{\partial^{2} h}{\partial y^{2}}-B \nabla^{4} h
$$

Für die Koeffizienten $C_{0}(\theta)$ und $C_{1}(\theta)$ gilt allgemein ${ }^{3}$

$$
\begin{aligned}
& C_{0}(\theta)=J M^{(0)} \cos (\theta) \\
& C_{1}(\theta)=J \frac{\partial}{\partial \theta}\left(M^{(0)} \cos (\theta)\right)
\end{aligned}
$$

\footnotetext{
${ }^{3} \mathrm{Im}$ folgenden schreiben wir die Bewegungsgleichung (2.27) mit positiven Vorzeichen vor jedem Koeffizienten, mit Ausnahme der Terme der Diffusion und des viskosen Fließens. Der Grund dafür ist, dass Erosion, Ionenimplantation und Massenredistribution berücksichtigt werden, und daher können alle Koeffizienten positive und negative Komponenten haben. Der letzte Term enthält den immer positiven Koeffizienten für die Oberfächendiffusion $B$. Deswegen wurde das negative Vorzeichen für diesen Term gewählt, so dass es die Glättung der Oberfläche beschreibt.
} 
Die Koeffizienten $C_{0}(\theta)$ und $C_{1}(\theta)$ beschreiben jeweils die Erosions- und die Rippelgeschwindigkeit und ersetzen den ersten bzw. zweiten Term in Gl. 2.4. $M^{(0)}$ beschreibt hier den Sputteryiel $Y(\theta)$. Die Krümmungskoeffizienten $C_{11}(\theta)$ und $C_{0}(\theta)$ ersetzen die Koeffizienten $S_{x}$ bzw. $S_{y}$ in Gl. (2.4), jedoch bestehen sie hier aus jeweils zwei Komponenten

$$
\begin{aligned}
& C_{11}(\theta)=C_{11}^{\text {eros. }}+C_{11}^{\text {redis. }} \\
& C_{22}(\theta)=C_{22}^{\text {eros. }}+C_{22}^{\text {redis. }}
\end{aligned}
$$

mit

$$
\begin{aligned}
& C_{11}^{\text {eros. }}=\frac{\partial}{\partial \theta}\left(M_{1, \text { eros. }}^{(1)} \cos (\theta)\right) \\
& C_{11}^{\text {redis. }}=\frac{\partial}{\partial \theta}\left(M_{1, \text { redis. }}^{(1)} \cos (\theta)\right) \\
& C_{22}^{\text {eros. }}=\cot (\theta) \cos (\theta) M_{1, \text { eros. }}^{(1)} \\
& C_{22}^{\text {redis. }}=\cot (\theta) \cos (\theta) M_{1, \text { redis. }}^{(1)}
\end{aligned}
$$

Die Komponenten $C_{11}^{\text {eros. }}$ und $C_{22}^{\text {eros. }}$ repräsentieren den Erosionseffekt nach BH-Theorie. Die Komponenten $C_{11}^{\text {redis. }}$ und $C_{22}^{\text {redis. }}$ stellen hier den Mass-Redistributionseffekt nach CVModell in $x$ - bzw. $y$-Richtung dar. Die Gleichung für die Wachstumsrate der Rippel 2.8 kann mit Hilfe der Koeffizienten $C_{11}$ und $C_{22}$ geschrieben werden als

$$
R=-C_{11} k_{x}^{2}-C_{22} k_{y}^{2}-D\left(k_{x}^{2}+k_{y}^{2}\right)^{2}
$$

In der ersten Anwendung des Kraterfunktionsformalismus haben Norris et al. mit Hilfe molekulardynamischer (MD) Simulationen die Momente von Erosions- und Redistributionskraterfunktion für die Bestrahlung von Silizium-Oberflächen mit 100 und $250 \mathrm{eV} \mathrm{Ar}{ }^{+}$ Ionen berechnet und konnten somit Einschätzungen über die Krümmungskoeffizienten in der Bewegungsgleichung bekommen [119]. Die Vorteile des CFF sind, dass sowohl der Erosions- als auch der Redistributionseffekt berücksichtigt wird. Außerdem basiert die Winkelabhängigkeit der Sputter-Erosion und Mass-Redistribution nicht mehr auf einfa- 
chen $\sin \theta$-Annährungen wie im BH- und CV-Modell [22, 28]. Dies ermöglicht zuverlässigere Bestimmungen von kritischen Winkeln, an denen die Musterbildung auftaucht oder sich die Orientierung der Rippelmuster ändert. Hinzu kommen weitere Vorteile des CFF durch die einfache und schnelle Bestimmung der Momente der Kraterfunktion mit Hilfe molekulardynamische Simulationen oder sogar Monte-Carlo Simulationen wie es in dieser Arbeit im Abschnitt (2.8) gezeigt wird.

\subsection{Erweiterungen der Modelle}

Die Theorie der Musterbildung, vor allem die Bradley-Harper-Theorie, wurde vielmals erweitert, sodass sie auf weitere Klassen von Materialien anwendbar ist und die Präzision der Vorhersage der Musterbildung erhöht. In der BH-Theorie wird ein exponentielles Wachstum der Oberflächenrauigkeit $\omega_{r m s}$ mit der Abhängigkeit $\omega \propto \exp (R \Phi)$ vorhergesagt. Diese Vorhersage stimmt nur für kurze Sputterzeiten (kleine Fluenzen) [58]. Für längere Sputterzeiten (große Fluenzen) tritt eine Sättigung des Rauhigkeitswachstums ein [47]. Um die experimentell bewiesene Sättigung des Amplitudenwachstums zu beschreiben, haben Cuerno et al. nicht-linearer Terme in der Bewegungsgleichung der Oberfläche hinzufügt [37, 38]. Zur Beschreibung von einkristallinen und mehrkomponentigen Materialien wurde die BH-Theorie durch die Einführung richtungsabhängiger Diffusionskonstanten erweitert, vorausgesetzt der Sputteryield der einzelnen Komponenten hängt nicht von der Zusammensetzung der Probe ab [134, 147].

Für diese Arbeit ist die Einführung vom Kraterfunktion-Formalismus und die Erweiterung des CV-Modells von besonderem Interesse. Außerdem wird in diesem Abschnitt die Rolle der Ionenimplantation bei der Musterbildung dargestellt.

\subsubsection{Die erosive Krümmungsabhängigkeit der Kraterfunktion}

Die im Abschnitt (2.5) eingeführten Kraterfunktion hängt eigentlich von der gesamten Form der Oberfläche ab, die die Ionen-Einschlagpunkte umgibt [118]. Da es jedoch nicht einfach ist, die Kraterfunktion für beliebig geformte Oberfläche mit Hilfe der MD Simulationen zu finden, wurde in vielen Anwendungen des CFF die Krümmungsabhängigkeit der Kraterfunktion vernachlässigt und einfach die Kraterfunktion für eine flache Oberfläche benutzt [78, 119, 120]. Auf der anderen Seite zeigen einige Studien, dass die Krümmungsabhängigkeit der Kraterfunktion eine signifikante Rolle spielen kann. Zum Beispiel haben M. L. Nietiadi und H. M. Urbassek MD Simulationen für die Bestrahlung von amorphen

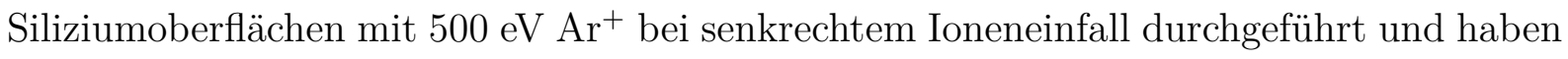
festgestellt, dass die Krater für gekrümmte Oberflächen sich wesentlich unterscheiden von 


\section{Theorie der Musterbildung}

denen für flache Oberflächen [116]. Außerdem haben Perkinson et al. in ihrer Arbeit [127] auf offensichtliche Inkonsistenzen in den Krümmungskoeffizienten $C_{11}$ und $C_{22}$ hingewiesen. Da der Ausdruck für $C_{11}$ (Gl. 2.32) eine Winkelableitung einer Funktion ist, die bei $\theta=0$ und $\pi / 2$ verschwindet, gilt für das Integral von $C_{11}$

$$
\int_{0}^{\pi / 2} C_{11}(\theta) d \theta=\left[-M_{1}^{(1)}(\theta) \cos (\theta)\right]_{0}^{\pi / 2}=0 .
$$

Zusätzlich folgt aus Gl. 2.32) und 2.34

$$
C_{11}(\theta)=\frac{d}{d \theta}\left(C_{22} \tan (\theta)\right)
$$

Die experimentellen Daten von Perkinson et al. für die Fälle $1 \mathrm{keV} \mathrm{Ar}^{+} / \mathrm{Si}$ und $\mathrm{Kr}^{+} / \mathrm{Ge}$ erfüllen jedoch die Gleichungen (2.37) und (2.38) nicht [127]. Die tatsächlichen Werte von $C_{11}$ und $C_{22}$ sind größer als die theoretischen Werte, die durch die Beziehungen (2.32) und 2.34 bestimmt wurden (Abbildung 2.8). Auch die Ergebnisse von Norris et al. haben einige zweifelhafte Aspekte [119]. Denn für Einfallswinkel $\theta$ unter einen kritischen Wert $\theta_{c}$ bleibt die Oberfläche flach. Für $\theta>\theta_{c}$ entwickeln sich Rippel-Muster mit der Ionenbestrahlung.
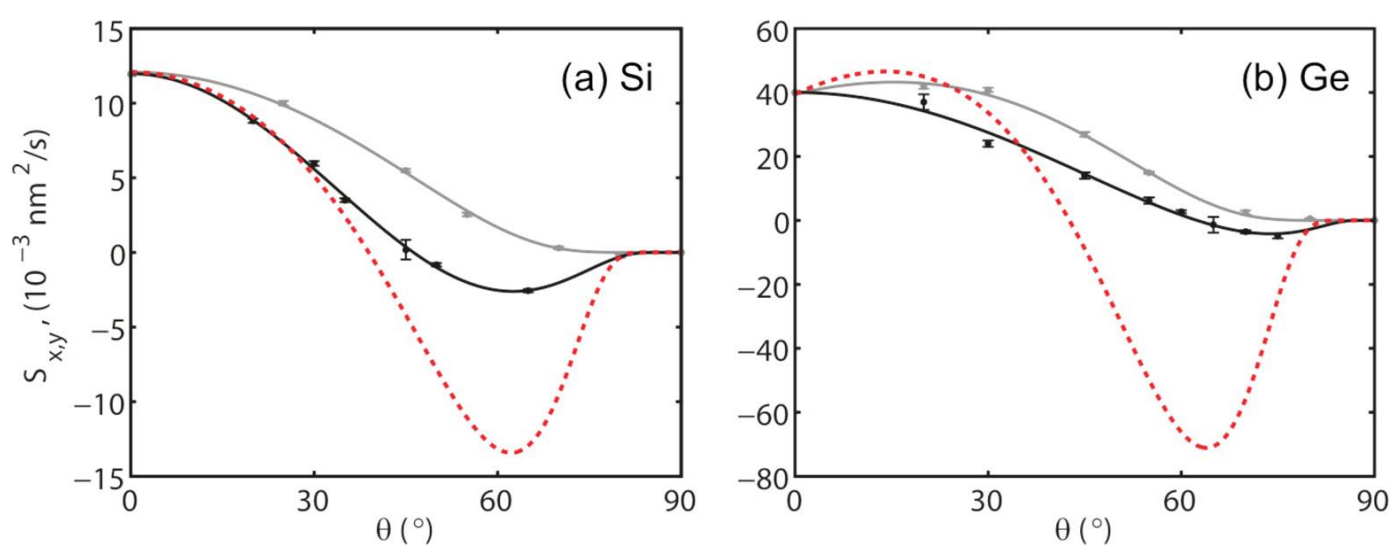

Abb. 2.8.: Die Krümmungskoeffizienten $C_{11}\left(S_{x}\right)$ und $C_{22}\left(S_{y}\right)$ als Funktion der Einfallswinkels: Die experimentellen Werte von $C_{11}$ (schwarze Kreise) und $C_{22}$ (graue Kreise) wie berichtet in [8, 93] für $\mathrm{Ar}^{+} / \mathrm{Si}$ (a) und in [9] für $\mathrm{Kr}^{+} / \mathrm{Si}$ (b). Die rote gestrichelte Linie ist die Voraussage von $C_{11}$ mit Hilfe Gl. (2.38) [127].

Allerdings war der experimentelle Wert von $\theta_{c}$ für $250 \mathrm{eV} \mathrm{Ar}^{+} / \mathrm{Si}$ (in diesem Fall 48) ungefähr $10^{\circ}$ größer als der theoretische Wert. Außerdem haben Norris et al. einen Wert für die gemessene Rippel-Wellenlänge für $\theta=50^{\circ}$, der etwa doppelt so groß wie der theore- 
tische Wert ist. Der Unterschied zwischen der gemessenen und theoretischen Wellenlänge sinkt erst ab einem Winkel von $65^{\circ}$. Schließlich wurde experimentell einen Übergang von parallelen Rippeln zu senkrechten Rippeln für flache Einfallswinkel beobachtet, jedoch wurde kein solcher Übergang durch die Eingabe der MD Ergebnisse von Norris et al. in den Gleichungen 2.32) und 2.34 gefunden [119]. All dies deutet darauf hin, dass die Berücksichtigung der Krümmungsabhängigkeit der Kraterfunktion von großer Bedeutung sein kann. Aus diesem Grund haben M. Harrison und M. Bradley den CFF so erweitert, dass er die Anhähngigkeit der Kraterfunktion von der Krümmung der Oberfläche an der Stelle des Ionen-Einschlags beinhaltet. Für eine komplette Herleitung der Krümmungskoeffizienten sei auf [61] verweisen. Laut Harrison und Bradley gelten für die Koeffizienten $C_{11}$ und $C_{22}$ die folgenden Beziehungen:

$$
\begin{aligned}
& C_{11}(\theta)=C_{11}^{\text {eros. }}+C_{11}^{\text {redis. }}+T_{11} \\
& C_{22}(\theta)=C_{22}^{\text {eros. }}+C_{22}^{\text {redis. }}+T_{22}
\end{aligned}
$$

Hier repräsentieren $T_{11}$ und $T_{22}$ die Krümmungsabhängigkeit der Kraterfunktion in $x$ bzw. $y$-Richtung und sind gegeben durch

$$
T_{i i}=-\cos (\theta)\left[\frac{\partial M_{K_{i i}}}{\partial K_{i i}}\right]_{K_{i i}=0}, \quad \text { mit } i=\{1,2\} \text {. }
$$

mit

$$
\begin{gathered}
M_{K_{i i}}=\iint F(x, y, \theta, K) d x d y \\
K_{11}=\frac{\partial^{2} h}{\partial x^{2}} ; K_{22}=\frac{\partial^{2} h}{\partial y^{2}}
\end{gathered}
$$

Der erweiterte CFF liefert die exsakten Koeffizienten für das Sputter-Modell von Sigmund und reduziert sich auf die von Norris et al. gegebenen Ergebnisse (Gl. 2.30 und 2.31) nur bei der Vernachlässigung der Krümmungsabhängigkeit der Kraterfunktion $T_{i i}$. Ist die Krümmung $K_{i i}$ am Anfang null und danach negativ, dann ist $M_{K_{i i}}$ eine abnehmende Funktion von $K_{i i}$ für $i=1$ und 2. In diesem Fall ist $T_{i i}$ positiv und führt zu einem stabilisierenden Beitrag (Glättung der Oberfläche). Der Glättungseffekt von $T_{i i}$ erhöht den von Norris et al. [119] berechneten theoretischen Wert von $\theta_{c}$ und somit auch die Rippel- 


\section{Theorie der Musterbildung}

Wellenlänge, da die Wellenlänge proportional $\mathrm{zu}\left|C_{11}\right|^{-1 / 2}$ ist (Gl. 2.9). Im Gegensatz dazu ist $K_{i i}$ am Anfang null und danach positiv, dann ist $T_{i i}$ negativ und hat einen destabilisierenden Effekt, der zur Aufrauung der Oberfläche bzw. Musterbildung führt. Allerdings der Bedarf an einem stabilisierenden Beitrag, der die Inkonsistenzen in den theoretischen Werte von $C_{11}$ und $C_{22}$ erklärt, bleibt in dem Fall vorhanden und führt uns zur Erweiterung des CV-Modells, die im nächsten Abschnitt dargestellt wird.

\subsubsection{Das erweiterte Carter-Vishnyakov Modell}

Eine wesentliche Annahme im CV-Modell [28] ist, dass die Höhenentwicklung einer bestrahlten Oberfläche nicht von ihrer Dicke abhängt. Diese Annahme wurde durch die Theorie von Oron et al. [123], die eine viskose Flüßigkeit auf ein festes Interface beschreibt, gerechtfertigt. Außerdem wird im CV-Model nur der laterale Massentransport $\delta_{\|}$(Gl. 2.12 berücksichtigt. Der nach innen gerichtete Massentransport ist in den vorherigen Studien vernachlässigt [40, 41, 93, 119]. Allerdings können diesen beiden Effekte eine signifikante Rolle bei der Musterbildung spielen und die Inkonsistenzen im CFF erklären. In diesem Abschnitt werden wichtige Erweiterungen des CV-Modells durch die Einführung von sowohl der Dickenabhängigkeit der bestrahlten Oberfläche als auch die Einführung des inneren Massentransports dargestellt [122].

\section{Einführung der Dickenabhängigkeit der bestrahlten Oberfläche}

Bei der Betrachtung des ionenstrahlinduzierten viskosen Fließens kann die Bewegungsgleichung der Oberfläche in einem Koordinatensystem $(u, v, w)$ geschrieben werden als:

$$
\frac{d h}{d t}=\left[C_{11}^{\text {eros. }}+C_{11}^{\text {redist. }}\right] \frac{\partial^{2} h}{\partial u^{2}}+\left[C_{22}^{\text {eros. }}+C_{22}^{\text {redist. }}\right] \frac{\partial^{2} h}{\partial v^{2}}-F_{S, \text { rad }} d^{3} \nabla^{4} h-B \nabla^{4} h .
$$

Da die ersten zwei Terme in der Bewegungsgleichung (2.27) kein Effekt auf die Musterbildung haben, werden sie hier nicht berücksichtigt. $F_{S, \text { rad }}$ beschreibt das ionenstrahlinduzierte viskose Fließen in einer dünnen Schicht mit der Dicke $D$ [122]. Bei einer Bestrahlung bei Raumtemperatur kann typischerweise die thermische Oberflächendiffusion $B$ vernachlässigt werden. Für eine gegebene Oberflächenviskosität $\eta_{S}$ und freie Oberflächenenergie $\gamma$ erhalten wir

$$
F_{S, \text { rad }}\left(\theta, E_{\text {ion }}\right)=\frac{\gamma \cdot J \cdot \cos \theta}{3 \eta_{S, \text { rad }}\left(\theta, E_{\text {ion }}\right)},
$$

wobei der Ionenfluss $J$ in einer auf den Ionenstrahl senkrechten Ebene gemessen wird [122, 141, 144]. In der Literatur wird oft angenommen, dass die bestrahlte Schicht eine 
konstante Dicke hat und somit ist $F_{S, r a d} \cdot D^{3}$ konstant. Jedoch hängt die Dicke einer bestrahlten Oberfläche in der Tat von der Ionenenergie und dem Einfallswinkel ab [9, 119]. Hans Hofsäss hat in seiner Arbeit [70, 71] das CV-Modell erweitert, indem er die Winkelabhähngigkeit der Schichtdicke bewiesen und in der Bewegungsgleichung berücksichtigt hat. Die durch die einfallenden Ionen entstehenden Kollisionskaskaden erzeugen einen atomaren Massentransport in der oberen Schicht des Festkörpers. Für einen Einfallswinkel $\theta$ ist $\delta_{u}(\theta)$ der laterale Massentransport pro Ion.

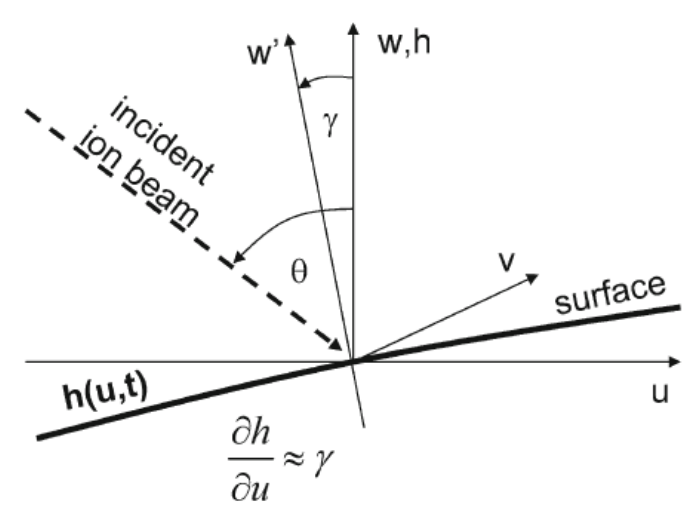

Abb. 2.9.: Schematische Darstellung eines Ionenstrahls auf eine geneigte Oberfläche [70].

Der laterale Massentransport für eine lokale Oberfläche, die um einen kleinen Winkel $\gamma$ im Bezug auf die mittlere Oberfläche geneigt ist, ist in dem Fall $\delta_{u}(\theta-\gamma)$ (Abbildung 2.9 und die mittlere Schichtdicke gemessen von der Oberfläche, in der der atomare Massentransport stattfindet, ist $D(\theta-\gamma)$. Die mittlere Schichtdicke $D$ ist nicht direkt mit der Ionen-Reichweite verbunden, sondern ist berechnet als die mittlere Tiefenverteilung von rückgestoßenen Atomen. Die die Ionen-Reichweite und die Tiefenverteilung der rückstoßenen Atome können sich, abhängig von der Ionenenergie und der Atommasse des Targets, erheblich unterscheiden. Die starke Abhängigkeit der Schichtdicke $D$ von dem Einfallswinkel $\theta$ hat Hofsäss in Abbildung 2.10 gezeigt. Die Abbildung zeigt die Tiefenverteilung der rückgestoßenen Atome und die Schichtdicke $D(\theta)$ berechnet mit dem Monte-Carlo Simulationsprogramm SDTrimSP für $10 \mathrm{keV}$ Xe auf Si. Zu bemerken ist, dass die Dicke $D(\theta)$ bei großen Einfallswinkel nicht einfach $\cos (\theta)$ folgt. Der Grund dafür ist, dass der innere Massentransport, der durch elastisch reflektierte Ionen verursacht wird, bei großen Einfallswinkel dominiert. Allerdings kann die Schichtdicke $D(\theta)$ mit der Formel $D(\theta)=D_{0}+D_{1} \cdot \cos (\alpha \cdot \theta)$ approximiert werden [70]. 

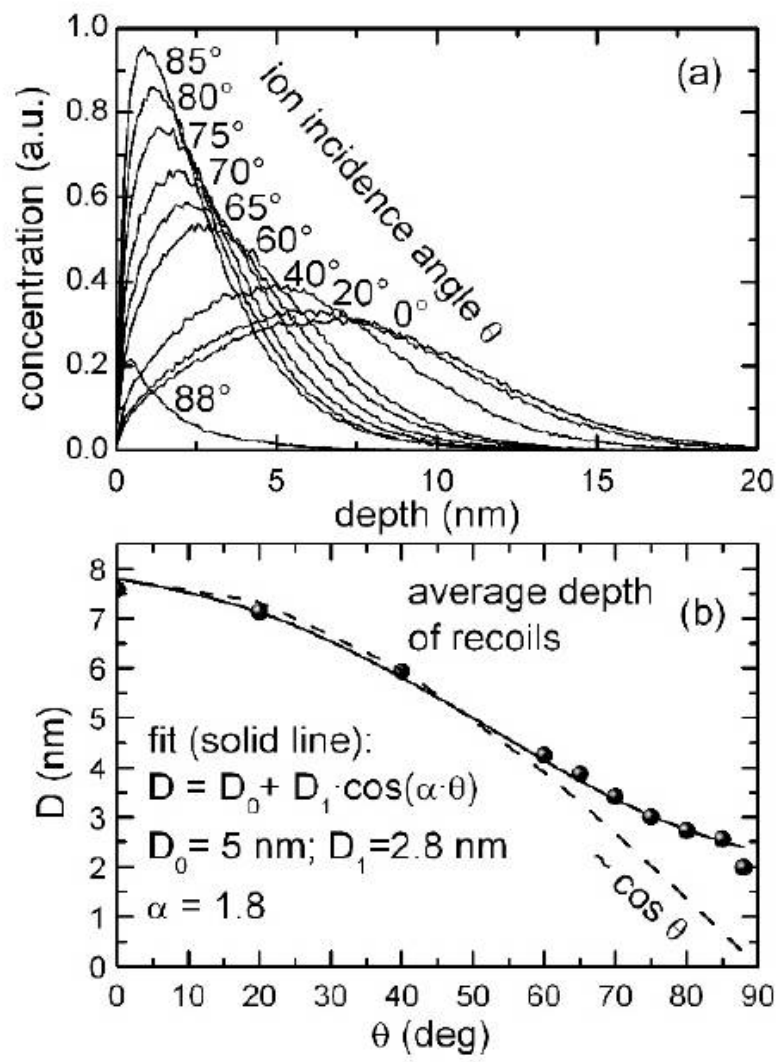

Abb. 2.10.: (a). Tiefenverteilung der rückgestoßenen Atome berechnet mit dem Simulationsprogramm SDTrimSP. (b). Die mittlere Tiefe der rückgestoßenen Atome als Funktion vom Einfallswinkel. Die gestrichelte Kurve zeigt eine $\cos \theta$ Abhängigkeit, die durchgezogene Kurve ist ein Fit mit der Formel, die in der Abbildung gezeigt wird [70].

Experimentell ist die Winkelabhängigkeit der bestrahlten Schichtdicke $D(\theta)$ anhand einer TEM-Querschnittsaufnahme von Siliziumoberfläche bestrahlt mit $120 \mathrm{keV} \mathrm{Ar}{ }^{+}$Ionen bei $60^{\circ}$ zu sehen (Abbildung 2.11). Für die Randbedingungen an der Interface wurden im CV-Modell die Haftbedingung $\left(v_{u}=v_{v}=0\right)$ und die Randbedingung „kein Transfer“ mit $\left(v_{w}=0\right)$ angenommen. Die von Hofsäss erstellten Simulationen in Abbildung 2.13) bestätigen die Haftbedingung am Interface. Abbildung (2.13) zeigt die laterale Schichtgeschwindigkeit als Funktion der Tiefe für $10 \mathrm{keV}$ und $1 \mathrm{keV} \mathrm{Ar}{ }^{+}$auf Si. Die Geschwindigkeit nimmt exponentiell mit der Tiefe ab und ist vernachlässigbar klein für $d>2 D$. Für die laterale Geschwindigkeit der Atome am Interface innerhalb einer viskosen Schicht gilt die Randbedingung $v_{u}=v_{v}=0$. Jedoch gilt hier die zweite Randbedingung mit „kein Transfer" nicht. Denn für eine Oberfläche, die ständig mit Ionen bestrahlt wird, müssen wir ein Interface $b(u, v, t)$ zum Austausch von Atomen zwischen dem Substrat und der bestrahlten viskosen Schicht zulassen. 


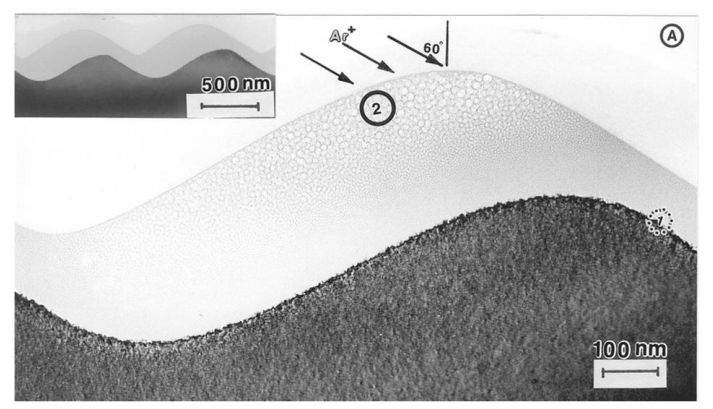

Abb. 2.11.: TEM-Aufname einer Siliziumoberfläche bestrahlt mit $120 \mathrm{keV} \mathrm{Ar}{ }^{+}$bei $60^{\circ}$. Der helle Bereich zeigt die bestrahlte viskose Oberfläche. Der dunkle Bereich zeigt das unbestrahlte Interface [36].

Der Grund dafür ist, dass das Interface $b(u, v, t)$ und die Oberfläche $h(u, v, t)$ durch die Größe der Kollisionskaskade verknüpft sind. Die Oberfläche $h$ und das Interface $b$ haben die folgende Beziehung:

$$
b(u, v, t)=h(u, v, t)-d(u, v, t) .
$$

Abbildung 2.12 zeigt eine durch Ionen bestrahlte viskose Oberfläche auf ein nicht bestrahltes festes Substrat. Die Dicke $h_{0}=D(\theta)$ ist für die flache Oberfläche, die mit Ionen bei einem Einfallswinkel $\theta$ bestrahlt wird. Die obere Kurve ist das Höhenprofil $h(u, t)$ einer mit Rippel geformte Oberfläche. Die untere Kurve ist das Interface $b(u, t)=$ $h(u, t)-D(u, t)$. Die vertikalen Pfeile zeigen die Positionen an, an denen die Schichtdicke gleich der Dicke $h_{0}$ ist. Dazwischen sind Bereiche, in denen die Schichtdicke entweder größer oder kleiner (abhängig vom lokalen Einfallswinkel) als $h_{0}$ ist. Als eine Annährung hat Hofsäss die Dicke der bestrahlten viskosen Schicht mit $d(\theta) \approx 2 \cdot D(\theta)$ approximiert, wobei $D(\theta)$ die mittlere Tiefenverteilung der rückgestoßenen Atome ist. Mit dieser Annährung finden etwa $95 \%$ aller Rückstoß-Ereignisse innerhalb $d(\theta)$ statt, wie die Abbildung (2.13) zeigt. Der durch die Kollisionskaskaden verursachte Massentransport entlang der $u$-Richtung kann als eine durchschnittliche Driftgeschwindigkeit $v_{\text {drift }}$ beschrieben werden. Die Driftgeschwindigkeit ist in Ref. [70] hergeleitet und gegeben durch:

$$
v_{\mathrm{drift}}=\frac{J}{n} \frac{\delta_{u}\left(\theta-\gamma_{u}\right)}{d\left(\theta-\gamma_{u}\right)} \cdot \cos \left(\theta-\gamma_{u}\right)
$$

Hier ist $n$ die Atomdichte des Targets. Im Fall eines Geschwindigkeitsfelds $v_{u}(w)$ innerhalb der Oberfläche $h$ und dem Interface einer festen Substrat $b$ kann die Driftgeschwindigkeit 


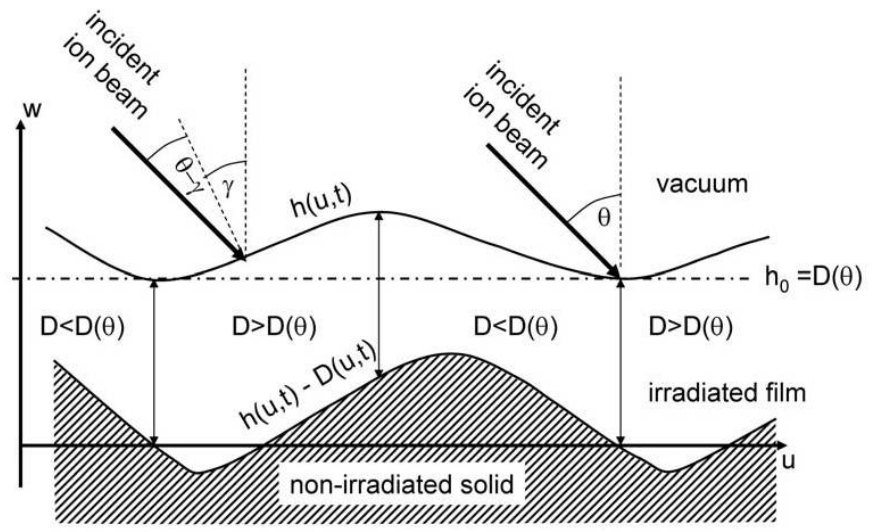

$\boldsymbol{A} \boldsymbol{b} \boldsymbol{b}$. 2.12.: Skizze einer mit Ionen bestrahlten viskosen Oberflächenschicht. Die Dicke $h_{0}$ ist für eine flache Oberfläche bestrahlt mit Ionen bei einem Einfallswinkel $\theta$. Die obere Kurve ist das Höhenprofil $h$ einer mit Rippel geformten Oberfläche. Die untere Kurve ist das Interface $d[70]$.

auch geschrieben werden als:

$$
v_{\mathrm{drift}}=\frac{1}{d} \int_{b}^{h} v_{u}(w) d w
$$

Die kinematische Randbedingung an der Oberfläche $h(u, v, t)$ ist ähnlich wie Gl. (2.12a) in Ref. [123] gegeben:

$$
\frac{d h}{d t}=v_{w}(h)-v_{u}(h) \frac{\partial h}{\partial u}-v_{v}(h) \frac{\partial h}{\partial v},
$$

wobei $v_{i}(h)$ die Schichtgeschwindigkeit ist, die ähnlich wie eine hochviskose Flüssigkeit fließt. Durch eine lineare Entwicklung der Dicke $d(\theta)$ an der Stelle $(\theta-\gamma)$ erhalten wir:

$$
\begin{aligned}
d(\theta-\gamma) & =d(\theta)-\left[\frac{\partial d(\theta-\gamma)}{\partial(\theta-\gamma)}\right]_{\gamma=0} \cdot \gamma \\
& =d(\theta)-\left[\frac{\partial d(\theta-\gamma)}{\partial(\theta-\gamma)}\right]_{\gamma=0} \cdot \frac{\partial h}{\partial u}
\end{aligned}
$$

Für eine Krümmung $\frac{\partial^{2} h}{\partial u^{2}}$ erhalten wir für die Ableitung $\frac{\partial d}{\partial u}$ die folgende Beziehung:

$$
\frac{\partial d}{\partial u}=-\frac{\partial d}{\partial \theta} \frac{\partial^{2} h}{\partial u^{2}}
$$

Da $\frac{\partial d}{\partial \theta}$ für alle Einfallswinkel negativ ist, nimmt die Dicke $d$ im Fall einer positiven Krüm- 
mung zu und im Fall einer negativen Krümmung ab, in Übereinstimmung mit der experimentellen Beobachtung in Abbildung (2.11) [70]. Für die $v$-Richting neigt sich die lokale Oberflächennormale um einen kleinen Winkel $\gamma_{v}$. Der lokale Einfallswinkel in $v$-Richtung ist somit $\theta_{v}=\arccos \left(\sqrt{1+\gamma_{v}^{2}} \cdot \cos \theta\right) \approx 0$. Deswegen kann man die Änderung der Schichtdicke in $v$-Richtung mit $\frac{\partial d}{\partial v} \approx 0$ vernachlässigen [71].
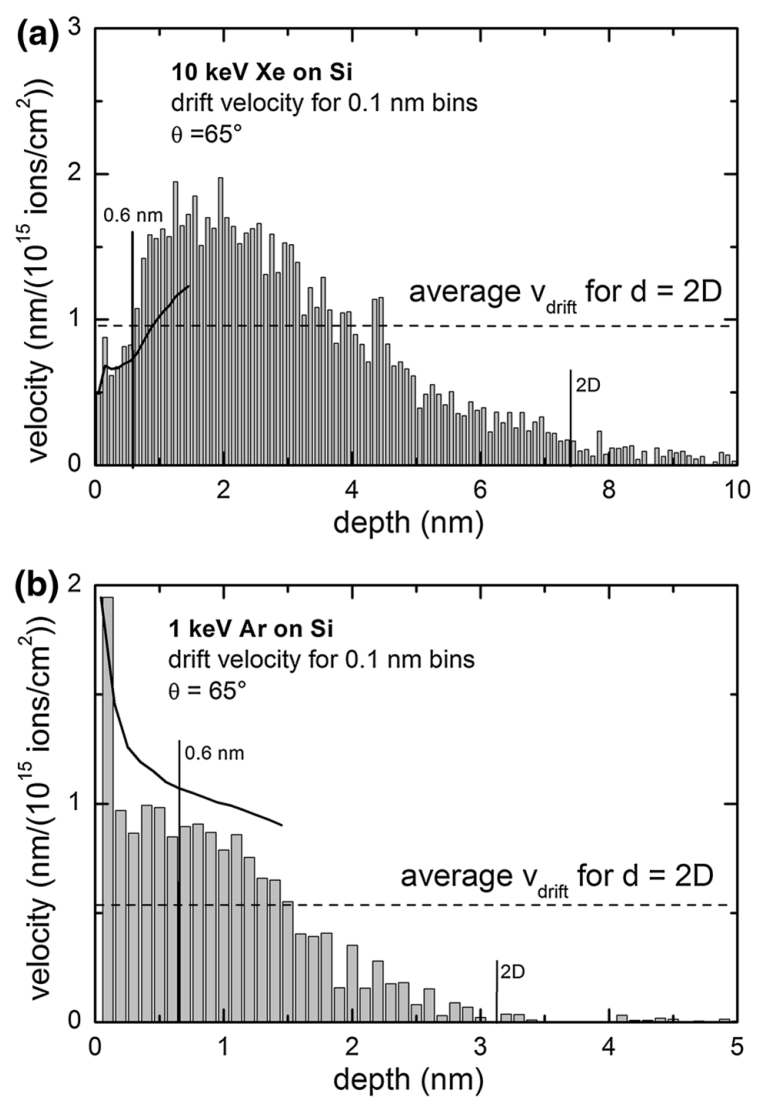

Abb. 2.13.: Driftgeschwindigkeit der rückgestoßenen Atomen als Funktion der Tiefe für (a) $10 \mathrm{keV} \mathrm{Xe}{ }^{+}$auf Si bei $65^{\circ}$ und (b) $1 \mathrm{keV} \mathrm{Ar}^{+}$auf Si bei $65^{\circ}$, berechnet mit SDTrimSP [42]. Das Tiefeninkrement ist $0.1 \mathrm{~nm}$.

Die zeitliche Abhängigkeit der Schichtdicke $d$ an einer gegebenen Stelle $(u, v)$ taucht nur auf im Fall einer lateralen Geschwindigkeit an der Oberflälche zusammen mit einer Oberflächenkrümmung und ist gegeben durch

$$
\frac{\partial d}{\partial t}=-v_{u, \text { film }}(h) \frac{\partial d}{\partial u}=v_{u, \text { film }}(h) \frac{\partial d}{\partial \theta} \frac{\partial^{2} h}{\partial u^{2}} .
$$

Hier nimmt die Dicke an einer gegebenen Stelle $(u, v)$ mit der Zeit für eine positive Drift- 


\section{Theorie der Musterbildung}

geschwindigkeit und eine positive Krümmung ab und für eine negative Krümmung zu [71]. Wie in Abschnitt (2.4) erwähnt, geht man beim ionenstrahlinduzierten viskosen Fließen von einem Relaxationsprozess aus, der dafür sorgt, dass die Dichte der bestrahlten Schicht konstant bleibt (inkompressible Schicht) [123]. Für die bestrahlte Schicht gilt die Kontinuumsgleichung mit den lokalen Geschwindigkeiten $v_{u}, v_{v}$ und $v_{w}$ :

$$
\frac{\partial v_{w}}{\partial w}=-\frac{\partial v_{u}}{\partial u}-\frac{\partial v_{v}}{\partial v}
$$

Durch Integration dieser Gleichung von Interface $b$ bis Oberfläche $h$ erhalten wir mit Hilfe partieller Integration die folgende Gleichung:

$$
v_{w}(h)=-\frac{\partial}{\partial u} \int_{b}^{h} v_{u} d w-\frac{\partial}{\partial v} \int_{b}^{h} v_{v} d w+v_{u}(h) \frac{\partial h}{\partial u}+v_{v}(h) \frac{\partial h}{\partial v}
$$

Die Bewegungsgleichung für eine mit Ionen bestrahlte Oberfläche (Gl. 2.49) muss die Zeitabhängigkeit der Schichtdicke $d$ (Gl. 2.51) berücksichtigen. Im Fall der Zeitabhängigkeit von $d$ bewegt sich das Interface mit der Geschwindigkeit $\frac{\partial d}{\partial t}$ bezüglich der Oberfläche $h$ und reduziert sich somit die Geschwindigkeit $v_{w}(h)$ um den gleichen Betrag

$$
v_{w}(h)_{\text {dynamisch }}=v_{w}(h)-\frac{\partial d}{\partial t}=v_{w}(h)-v_{u}(h) \frac{\partial d}{\partial \theta} \frac{\partial^{2} h}{\partial u^{2}} .
$$

Das Integral über die Geschwindigkeit $v_{u}(w)$ innerhalb der Schichtdicke $d$ ist nichts anderes als die Driftgeschwindigkeit, gegeben in Gl. 2.48), multipliziert mit $d$. Das Integral über $v_{v}(h)$ innerhalb der Schicht $d$, die um einen kleinen Winkel $\gamma_{v}=-\frac{\partial h}{\partial v} \mathrm{im}$ Bezug zu der globalen Oberfläche geneigt ist, ist

$$
\int_{0}^{d} v_{v} d w^{\prime}=-v_{\mathrm{drift}} \cdot d \cdot \cot (\theta) \cdot \gamma_{v}=-v_{\mathrm{drift}} \cdot d \cdot \cot (\theta) \cdot \frac{\partial h}{\partial v}
$$

mit

$$
v_{v}=-v_{u} \cot (\theta) \frac{\partial h}{\partial v}
$$

Beim Einsetzen der Gl. 2.53 in 2.54 erhalten wir:

$$
\frac{\partial}{\partial u}\left(v_{\text {drift }} d\right)=\frac{J}{n} \frac{\partial}{\partial u}\left(\delta_{u}(\theta) \cos (\theta)\right)
$$


Aus einer linearen Entwicklung von $v_{\text {drift }}$ in Analogie zu Gl. 2.50 folgt:

$$
\frac{\partial}{\partial u}\left(\delta_{u}(\theta) \cos (\theta)\right)=-\left(-\sin (\theta) \cdot \delta_{u}(\theta)+\cos (\theta) \frac{\partial \delta_{u}}{\partial \theta}\right) \frac{\partial^{2} h}{\partial u^{2}}
$$

Bei der Kombination von Gl. 2.49) und Gl. (2.54) und der Vernachlässigung des kleinen Beitrags $v_{v}(h) \frac{\partial h}{\partial v} \approx 0$ erhalten wir für die Bewegungsgleichung der Oberfläche:

$$
\frac{\mathrm{d} h}{\mathrm{~d} t}=C_{u}^{\text {redist. }} \frac{\partial^{2} h}{\partial u^{2}}+C_{v}^{r e d i s t} \cdot \frac{\partial^{2} h}{\partial v^{2}}-v_{u}(h) \frac{\partial d}{\partial \theta} \frac{\partial^{2} h}{\partial u^{2}} .
$$

mit

$$
\begin{gathered}
C_{u}^{\text {redist. }}=\frac{J}{n}\left(-\sin (\theta) \delta_{u}(\theta)+\cos (\theta) \frac{\partial \delta_{u}}{\partial \theta}\right) \\
C_{v}^{\text {redist. }}=\frac{J}{n} \delta_{u}(\theta) \cos (\theta) \cdot \cot (\theta) .
\end{gathered}
$$

Bei der Berücksichtigung der Dickenabhängigkeit der bestrahlten Oberfläche kann der Krümmungskoeffizient $C_{11}$ in Gl. 2.39) ergänzt werden und zwar in der folgenden Weise:

$$
C_{11}(\theta)=C_{11}^{\text {eros. }}+C_{11}^{\text {redis. }}+T_{11}+D_{11}
$$

mit

$$
D_{11}=-v_{u}(h) \frac{\partial d}{\partial \theta}
$$

Das Abnehmen der Schichtdicke $d$ mit zunehmendem Einfallswinkel ergibt einen zusätzlichen Term $D_{11}$ für den Koeffizient $C_{11}^{\text {redist. }}$. Dieser Term ist immer positiv, da $\frac{\partial d}{\partial \theta}<0$. Daher führt $D_{11}$ zur Stabilisierung der Oberfläche in $u$-Richtung (parallel zur Ionenstrahlrichtung) vor allem für Einfallswinkel zwischen $30^{\circ}-65^{\circ}$ und eliminiert somit die von Perkinson et al. beobachtete Inkonsistenz (siehe Abschnitt 2.6.1) [70, 71, 127].

\section{Einführung der inneren Massenrelaxation}

Im CV-Modell und den darauf basierenden Studien wurde bisher nur der laterale Massentransport $\delta_{u}$ berücksichtigt. Um die Dichte der bestrahlten viskosen Schicht konstant beizubehalten, wurde im CV- Modell angenommen, dass der nach innen gerichtete Massentransport sich vollständig entspannt. Deswegen hat er kein Effekt auf die Musterbildung und kann vernachlässigt werden. Hofsäss hat jedoch die Rolle des inneren Massen- 


\section{Theorie der Musterbildung}

transports an einem Gedankenexperiment veranschaulicht [70]. Er betrachtet eine flache Oberfläche mit Ionen-Fluenz $\Phi=n^{2 / 3}$, die senkrecht auf einen Festkörper mit Atomdichte $n$ einfällt. Die Ionen-Fluenz verursacht einen nach innen gerichteten mittleren Massentransport mit dem Abstand $-\delta_{w}$ pro Ion. Für eine Fluenz $\Phi=n^{2 / 3}$ wird sich eine atomare Oberflächenschicht nach innen um den Abstand $-\delta_{w}$ verschoben und sich dann zurück in Richtung Oberfläche um $+\delta_{w}$ entspannen. In der gleichen Zeit wird jedoch die Oberfläche durch den Ionenstrahl mit einem mittleren Sputteryield $Y$ erodiert. Der Erosionseffekt entspricht einer Erosionstiefe $\left(n^{2 / 3} \cdot Y\right) / n=Y / n^{1 / 3}$. Die Rückwärts-Entspannung wird in diesem Fall um $Y / n^{1 / 3}$ kleiner sein. Jetzt betrachten wir eine mit Rippel geformte Oberfläche, die durch Ionen mit einem Einfallswinkel $\theta$ bezüglich der Oberflächennormale bestrahlt wird (Abbildung 2.14). Die Oberfläche ist laut BH-Theorie [22] mit einer mittleren Rate

$$
v_{0}(\theta)=\left(\frac{J}{n}\right) \cdot Y(\theta) \cdot \cos (\theta)
$$

erodiert und für eine Fluenz $\Phi \cdot \cos \theta=n^{2 / 3}$ um eine mittlere Erosionstiefe $Y / n^{1 / 3}$ nach unten verschoben. An der Stelle A in Abbildung (2.14) fällt der Ionenstrahl mit einem Winkel $\theta$ auf die Oberfläche ein. Der nach innen gerichtete Massentransport entspannt sich zurück in die gleiche Position $\mathrm{U}_{\mathrm{A}}$. Für den Punkt B mit einer positiven Steigung verursacht die Ionenbestrahlung einen lateralen $\left(+\delta_{u^{\prime}}\right)$ und inneren $\left(-\delta_{w^{\prime}}\right)$ Massentransport, wobei $\left(u^{\prime}, v^{\prime}, w^{\prime}\right)$ ein lokales Koordinatensystem ist. Der Massentransport entspannt sich aber zurück in Richtung neuer Position, die durch $u=u_{\mathrm{B}}+\left(Y / n^{1 / 3}\right) \cdot \gamma$ mit $\gamma=\partial h / \partial u$ gegeben ist. Der laterale Massentransport $\delta_{u}$ wird dann zu $\delta_{u}+\left(Y / n^{1 / 3}\right) \cdot \gamma$ geändert. Für den Punkt C mit einer negativen Steigung erfolgt die Entspannung des Massentransports in Richtung einer Position $u=u_{\mathrm{C}}-\left(Y / n^{1 / 3}\right) \cdot|\gamma|$ und $\delta_{u}$ ist zu $\delta_{u}-\left(Y / n^{1 / 3}\right) \cdot|\gamma|$ geändert. Deswegen muss man der folgende Term zusäzlich zur Gleichung (2.58) hinzufügen:

$$
\frac{\partial}{\partial u}\left(\frac{Y}{n^{1 / 3}} \cdot \frac{\partial h}{\partial u} \cdot \cos (\theta)\right) \approx \cos (\theta) \frac{Y}{n^{1 / 3}} \cdot \frac{\partial^{2} h}{\partial u^{2}} .
$$

Für die $v$-Richtung gilt:

$$
\frac{\partial}{\partial v}\left(\frac{Y}{n^{1 / 3}} \cdot \frac{\partial h}{\partial v} \cdot \cos (\theta)\right) \approx \cos (\theta) \frac{Y}{n^{1 / 3}} \cdot \frac{\partial^{2} h}{\partial v^{2}}
$$

Mit Hilfe Gl. 2.65) und (2.66) Kann man die die Krümmungskoeffizienten in Gl. (2.60) 


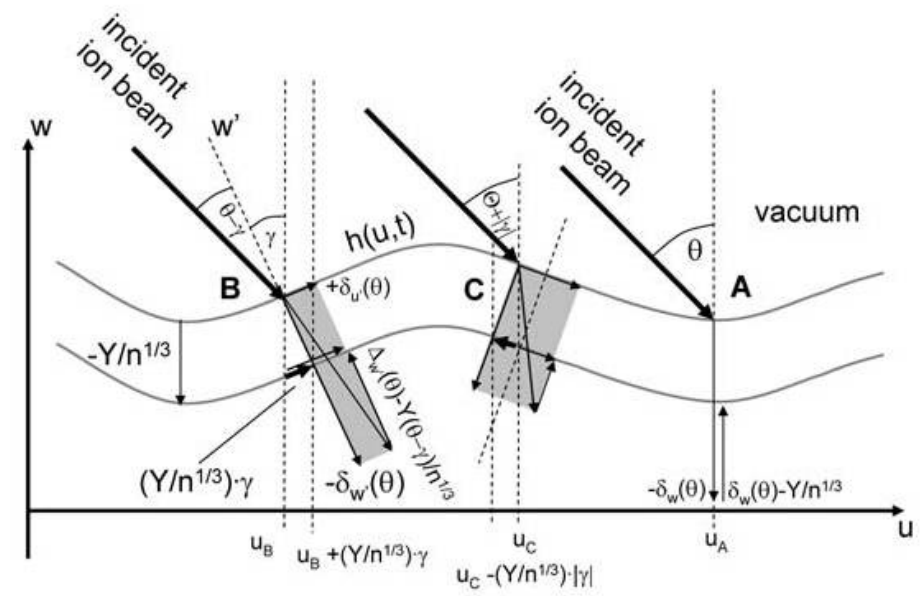

$\boldsymbol{A} \boldsymbol{b} \boldsymbol{b}$. 2.14.: Skizze eines ionenstrahlinduzierten Massentransports in einer dünnen Festkörperschicht mit der Atomdichte $n$ und für eine Fluenz $\Phi \cos \theta=n^{2 / 3}$. $Y / n^{1 / 3}$ ist die mittlere Erosionstiefe, A die Stelle, wo sich der Massentransport in die gleiche Position zurück entspannt. B ist die Stelle mit einer positiven Steigung und $\mathbf{C}$ mit einer negativen Steigung. Die langen dicken Pfeile zeigen die einfallend Ionen. Die langen dünnen Pfeile zeigen der Massentransport von einer imaginären atomaren Oberflächenschicht verschoben durch die Ionen-Fluenz um $-Y / n^{1 / 3}$. Die kurzen dicken Pfeile geben die Verschiebung der Distanz $Y / n^{1 / 3} \cdot \gamma$ an [70].

und 2.61 wie folgt umschreiben:

$$
\begin{gathered}
C_{u}^{\text {redist. }}=\frac{J}{n}\left[-\sin (\theta) \delta_{u}(\theta)+\cos (\theta)\left(\frac{\partial \delta_{u}}{\partial \theta}-\frac{Y}{n^{1 / 3}}\right)\right], \\
C_{v}^{\text {redist. }}=\frac{J}{n} \delta_{u}(\theta) \cos (\theta) \cdot\left(\cot (\theta)-\frac{Y}{n^{1 / 3}}\right) .
\end{gathered}
$$

Der Korrektorterm $Y(\theta) \cdot n^{-1 / 3}$ scheint klein zu sein, jedoch bei großen Winkel erreicht der Sputteryield $Y$ sein Maximum, während $\cot (\theta)$ sich Null annährt. Deshalb reduziert der Korrekturterm den kleinen stabilisierenden Beitrag von Massen-Redistribution bei großen Einfallswinkeln und führt möglischerweise zu einer senkrechten Rippelmuster [70].

\subsubsection{Die Rolle der Ionenimplantation}

In den meisten vorherigen Studien, die die ionenstrahlinduzierte Musterbildung untersucht haben, wurde angenommen, dass keine Ionen in die Oberflächenschicht implantiert werden. Neben den chemischen Effekten, die durch die Ionenstrahlen auftreten können, wird es einen Einfluss auf die Musterbildung durch den Einbau der Ionen selbst geben. 


\section{Theorie der Musterbildung}

Mehrere experimentelle Studien auf einfachen Substraten wie C, Si und Ge wurden mit anderen Ionen als Edelgasionen gemacht. Beispiele sind $\mathrm{O}^{+}$oder $\mathrm{O}_{2}^{+}$[14, 45, 77, , 90, 101104, 107, 132, 146], $\mathrm{C}^{+}$[14, 15, 109], $\mathrm{N}_{2}^{+}$[7, 11, 14, 139, 140], $\mathrm{Si}^{+}$[29, 30, 54] und $\mathrm{Au}^{+}$[108] Ionenstrahlen, sowie $\mathrm{Ga}^{+}$Ionen mit Hilfe fokussierten Ionenstrahl-Systeme. [55, 59, 80, 98, 129]. Ein ideales Beispiel für die Untersuchung der Rolle der Ionenimplantation ohne chemischen Einfluss wäre die Bestrahlung von Kohlenstoffschichten mit Kohlenstoff-Ionen oder Siliziumsubstraten mit Silizium-Ionen. Neulich haben Bradley und Hofsäss eine Musterbildungstheorie durch das Selbst-Sputtern mit der Berücksichtigung des Effekts der Ionenimplantation eingeführt [23]. Sie haben sich der Einfachheit halber auf Bestrahlung von einfachen Substraten mit Selbst-Ionen beschränkt und haben festgestellt, dass die Ionenimplantation ein stabilisierender Effekt entlang des projizierten Ionenstrahls bis zu einem kritischen Winkel hat. Eine kürzlich durchgeführte Studie von Hofsäss et al. hat auch gezeigt, dass die Ionenimplantation für Rippel-Muster mit Wellenvektor parallel zum projizierten Ionenstrahl einen stabilisierenden Beitrag bei kleinen Einfallswinkel und einen destabilisierenden Beitrag für Winkel über $45^{\circ}$ hat. Für Rippel-Muster mit Wellenvektor senkrecht zum projizierten Ionenstrahl hat die Implantation immer einen stabilisierenden Beitrag für alle Einfallswinkel [75]. Hofsäss et al. beschreiben die Ionenimplantation anhand des Kraterfunktionsformalismus (CFF). Die erforderlichen Momente der Implantationskraterfunktion wurden mit Hilfe des Monte-Carlo-Simulationsprogramms SDTrimSP [42] bestimmt. Der Einbau von Ionen in die Oberflächenschicht kann als ein negativer Sputteryield interpretiert und deswegen kann die Ionenimplantation ähnlich wie die Erosion in BH-Theorie beschrieben werden, mit der Bemerkung, dass der Effekt von Ionenimplantation das Gegenteil zur Ionenerosion ist. Daher kann der Koeffizient $C_{0}$ aus der Bewegungsgleichung im Fall der Ionenimplantation erweitert werden, und zwar durch Einbeziehung der gesamten Wachstumgeschwindigkeit, die durch die implantierten Ionen verursacht wird. Wir erhalten in Analogie zu Gl. 2.27):

$$
C_{0}=J\left(M_{\text {eros. }}^{(0)} \cos (\theta)+M_{\text {implant. }}^{(0)} \cos (\theta)\right)
$$

Die Rippelgeschwindigkeit ist in Analogie zu Gl. 2.29) gegeben

$$
C_{1}=C_{1}^{\text {eros. }}+C_{1}^{\text {implant }}
$$

mit

$$
C_{1}^{\text {implant. }}=J \frac{\partial}{\partial \theta}\left(M_{\text {implant. }}^{(0)} \cos (\theta)\right) .
$$


Der Effekt der Ioneninkorporation auf die Entwicklung der Oberfläche kann durch die Koeffizienten

$$
C_{11}^{\text {implant. }}(\theta)=S_{11}^{\text {implant. }}(\theta)+T_{11}^{\text {implant. }}(\theta)
$$

und

$$
C_{22}^{\text {implant. }}(\theta)=S_{22}^{\text {implant. }}(\theta)+T_{22}^{\text {implant. }}(\theta)
$$

beschrieben werden. Die Koeffizienten $S_{i i}$ und $T_{i i}$ sind definiert durch:

$$
\begin{aligned}
& S_{11}^{\text {implant. }}=J \frac{\partial}{\partial \theta}\left(M_{u, \text { implant. }}^{(1)} \cos (\theta)\right), \\
& S_{22}^{\text {implant. }}=J M_{u, \text { implant. }}^{(1)} \cos (\theta) \cot (\theta)
\end{aligned}
$$

und

$$
T_{i i}^{\text {implant. }}(\theta)=J \cos (\theta) \frac{\partial}{\partial K_{i i}}\left[M_{K_{i i}, \text { implant. }}\right]_{K_{i i}=0} .
$$

Ionenimplantation führt zu einem krümmungsabhängigen Koeffizient in der Bewegungsgleichung der Oberfläche und bewirkt eine destabilisierende Oberfläche für Muster mit Wellenvektor parallel zum projizierten Ionenstrahl erst dann, wenn die anderen Beiträge des Krümmungskoeffizients $C_{11}$ von der Erosion und Redistribution sich zu Null addieren oder positiv bleiben. Deswegen wird die Ionenimplantation ein wichtiger Beitrag zur Musterbildung liefern, z. B. im Fall einer Bestrahlung von Kohlenstofftarget mit leichten Ionen. Denn aufgrund der hohen Oberflächenbindungsenergie von Kohlenstoff wird der Sputteryield ziemlich klein. Außerdem führen die leichten Ionen nur zu einem kleinen Redistributionseffekt. Hofsäss et al. haben die experimentellen Daten für die Bestrahlung von amorphem Kohlenstoff mit ${ }^{12} \mathrm{C}^{+}$- und ${ }^{20} \mathrm{Ne}^{+}$-Ionen mit Monte-Carlo-Silumationen verglichen und haben einen signifikanten Unterschied im Verhalten der Musterbildung gefunden, der nur mit Hilfe der Ionenimplantation erklärt werden kann [75]. In den beiden Fällen sagen die Simulationen keine Musterbildung vorher, falls die Ionenimplantation vernachlässigt wird. Im Gegenteil wird ein paralleles Rippel-Muster vorhergesagt, wenn die Ionen in die Oberfläche des Targets implantiert werden. Die Ionenimplantation von $C^{+}$ Ionen hat wegen der großen Reichweite und des niedrigen Sputteryields einen signifikanten Einfluss auf die Instabilität der Oberfläche. Auf der anderen Seite ist der Effekt der implantierten $\mathrm{Ne}^{+}$Ionen aufgrund der kürzeren Reichweite und des größeren Sputteryields 


\section{Theorie der Musterbildung}

schwächer, was mit den experimentellen Daten übereinstimmt [75]. Allerdings kann der Implantation-Effekt in einigen Fällen durch chemische Effekte wie z. B. Phasenseparation, die im Abschnitt (2.9) dargestellt wird, verdeckt werden.

\subsection{Gemeinsame Betrachtung der Theorien}

Ein umfassendes Verständnis der Musterbildungsprozesse und somit eine quantitative Vorhersage der Musterbildung lässt sich gewinnen durch die Kombination von sowohl Erosionseffekt (BH-Theorie [22]) als auch Redistributionseffekt (CV-Modell [28]) inklusive der wichtigen Erweiterungen von Harrison-Bradley [61], Hofsäss [70, 71] und mit der Berücksichtigung des von Hofsäss et al. eingeführten Implantationseffekts [23, 75]. Für ein Koordinatensystem $(u, v, w)$ mit einer $w$-Achse parallel zur globalen Oberflächennormale und $u$-Achse entlang des projizierten Ionenstrahls kann die zeitliche Entwicklung der Oberfächenhöhe durch die folgende Gleichung beschrieben werden:

$$
\frac{d h}{d t}=C_{0}(\theta)+C_{1}(\theta) \frac{\partial h}{\partial u}+C_{11}(\theta) \frac{\partial^{2} h}{\partial u^{2}}+C_{22}(\theta) \frac{\partial^{2} h}{\partial v^{2}}-F_{S, r a d} d^{3} \nabla^{4} h-B \nabla^{4} h
$$

- $C_{0}(\theta)$ : ist die Erosionsgeschwindigkeit nach Gl. (2.69).

- $C_{1}(\theta)$ : ist die Rippel-Geschwindigkeit nach Gl. (2.70).

- $C_{i i}$ : ist der Krümmungskoeffizient mit $i=\{1,2\}$ und besteht aus den Effeke Erosion (Gl. 2.32 2.34 , Redistribution (Gl. 2.67 2.68 ) und Implantation (Gl. 2.72 2.73 (2) sowie der Krümmungsabhängigkeit der Kraterfunktion $T_{i i}$ (Gl. 2.41) und der Dickenabhängigkeit der bestrahlten Oberflächenschicht $D_{11}$ (Gl. 2.63).

$$
C_{11}^{\text {total }}=C_{11}^{\text {eros. }}+C_{11}^{\text {redist. }}+C_{11}^{\text {implant. }}+T_{11}+D_{11}
$$

und

$$
C_{22}^{\text {total }}=C_{22}^{\text {eros. }}+C_{22}^{\text {redist. }}+C_{22}^{\text {implant. }}+T_{22}
$$

- $F_{s, \text { rad }} \cdot d^{3}$ beschreibt das ionenstrahlinduzierte viskose Fließen nach Gl. 2.45.

- $B$ beschreibt die thermisch bedingte Oberlächendiffusion nach Gl. 2.5).

Die thermische Oberlächendiffusion wird normalerweise für eine Bestrahlung bei Raumtemperatur vernachlässigt. Wir erhalten für die Wachstumsrate mit einem Wellenvektor 
$q_{u}$ in Analogie zu Gl. 2.8):

$$
R\left(q_{u}\right)=-C_{11} \cdot q_{u}^{2}-F_{s, r a d} \cdot d^{3} \cdot q_{u}^{4}
$$

Der Term $F_{s, \text { rad }} \cdot d^{3}$ ist immer positiv und führt deswegen zu einer stabilen Oberflächenhöhe in $u$-Richtung. Wenn $C_{11}$ positiv ist, dann führt dieser Koeffizient ebenfalls zu einer stabilen Oberfläche in $u$-Richtung. Die Stabilität bedeutet in diesem Fall, dass die Oberfläche geglättet wird oder ein Wellenmuster in $v$-Richtung vorliegt. Entsprechend ist die Oberfläche in $u$-Richtung instabil für negative Werte von $C_{11}$. Die Instabilität bedeutet hier eine Wellenmuster in $u$-Richtung. Analog gilt dies für den Koeffizienten $C_{22}$. Der Wellenvektor $q_{u}$ kann mit einer maximalen Wachstumsrate und einem gegebenen Einfallswinkel wie folgt geschrieben werden:

$$
q_{u, \max }=\sqrt{\frac{-C_{11}}{2 F_{s, \text { rad }} d^{3}}} .
$$

Durch das Einsetzen von Gleichung (2.81) in 2.80 erhalten wir die maximale Wachstumsrate

$$
R_{\max }=\frac{C_{11}^{2}}{4 F_{s, r a d} d^{3}}
$$

Die Wellenlänge für eine maximale Wachstumsrate ist:

$$
\lambda=\frac{2 \pi}{q_{\max }}=2 \pi \sqrt{\frac{-2 F_{s, r a d} d^{3}}{C_{11}}} .
$$

Die Wachstumsamplitude ist dann gegeben durch:

$$
A(\Phi)=A_{0} \exp \left(\frac{R_{\max } \Phi}{J \cos \theta}\right) .
$$

Hofsäss et al. haben eine Wachstumsperiode $\tau$ und die entsprechende Fluenz $\Phi_{\tau}$ auf eine geneigte Oberfläche eingeführt [65]. Für $\frac{A\left(\phi_{e}\right)}{A_{0}} \equiv e$ gilt mit Hilfe Gleichung 2.82 :

$$
\Phi_{e}=\frac{J \cos \theta}{R_{\max }}=\frac{4 J F_{s, \text { rad }} d^{3}}{C_{11}^{2}} \cdot \cos \theta
$$

Die Fluenz $\Phi_{e}$, die die Wachstumsperiode $\tau$ charakterisiert, ist eine Maß dafür, bis zu welcher Fluenz das lineare Regime sich erweitern kann. Ein Übergang vom linearen zum nicht linearen Regime tritt für eine rms Rauhigkeit von etwa 8-10 nm auf [37, 38]. Aus- 
gehend von einer Rauhigkeit von $0.3 \mathrm{~nm}$ wird der Übergang nach etwa 3-4 Perioden erreicht. die Fluenz $\Phi_{e}$ kann ebenfalls als eine untere Grenzfluenz betrachtet wird, die für die Erzeugung eines schwachen Rippel-Muster erforderlich ist [65].

\subsection{Bestimmung der Krümmungskoeffzienten der Bewegungsgleichung}

Um die Krümmungskoeffizienten $C_{i i}$ in Gleichung (2.78) und (2.79) zu berechnen, werden Computersimulationen eingesetzt. Die Simulationsprogramme für Ionen-FestkörperWechselwirkung beruhen auf zwei Methoden. Die Methode der binären Kollisionen BCM! und die Methode der Molekulardynamik MD5. Bei der ersten Methode wird die Bewegung der Ionen als eine Serie von aufeinanderfolgenden binären Zusammenstößen betrachtet. Aus der Energie- und Impulserhaltung wird der Energieübertrag und die Streuung beim binären Stoß berechnet. Ist das Target als strukturlos betrachtet, d.h. amorph oder polykristallin, dann spricht man hier von Monte-Carlo-Simulationsprogrammen [145]. Die Methode der Molekulardynamik basiert auf der einfachen Strategie, dass die zeitliche Entwicklung eines Systems von Teilchen verfolgt werden kann, indem die newtonschen Bewegungsgleichungen des Systems gelöst werden [43, 145]. Ein weit verbreitetes BCMSimulationsprogramm ist SRIM ${ }^{6}$. Aufgrund seiner bequemen Benutzeroberfläche und seiner umfangreichen Datenbank ist SRIM heutzutage eines der beliebtesten Simulationsprogramme. Das Programm basiert auf das Simulationsprogramm TRIM $]^{7}$ und verwendet das hoch energetische ZBI 8 -Potential [161, 162]. SRIM ist optimal zur Berechnung von Ionenreichweite, Strahlenschäden und Energieverlusten. Bei Berechnung des Sputteryields zeigt SRIM jedoch eine Abweichung von den experimentellen Werten. Denn das Hauptproblem in SRIM ist die falsche Winkelverteilung der abgesputterten Atome für Targets mit niedrigen Z-Elementen ( $\mathrm{Z}<14$ und $\mathrm{Z}<18$ für SRIM-2008 und davor). Ein weiteres Problem steht im Zusammenhang mit der Winkelabhängigkeit des Sputteryields. Denn besonders für große Einfallswinkel ist der mit SRIM berechnete Sputteryield stark überschätzt [67]. Das Simulationsprogramm TRIDYN ${ }^{9}$ ist ähnlich wie TRIM aber verwendet die $\mathrm{KrC}^{10}$. Potentiale und ermöglicht dynamische Simulationen, indem es Stöchiometrieänderungen,

\footnotetext{
${ }^{4}$ Binary Collision Method

${ }^{5}$ Molecular Dynamics

${ }^{6}$ Stopping and Range of Ions in Matter

${ }^{7}$ Transport of Ions in Matter

${ }^{8}$ Ziegler-Biersack-Littmark

${ }^{9}$ TRIm simulation code including DYNamic composition

${ }^{10}$ Krypton-Carbon
} 
die durch Ionenimplantation und bevorzugtes Sputtern von Komponenten bei Targets aus Legierungen und Verbindungen verursacht werden, berücksichtigt [105]. Die meisten Analysen mit dem Kraterfunktionsformalismus CFF beruhen auf molekulardynamischen Simulationsdaten als Input. Jedoch werden oft in der MD-Simulationen niederenergetische Wechselwirkungspotentiale verwendet, außerdem sind MD-Simulationen sehr zeitaufändig. Deswegen sind sie beschränkt auf sehr niedrige Ionenenergie und einfache Substrate [79, 118, 119, 145].

Das in dieser Arbeit verwendete Simulationsprogramm ist SDTrimSP ${ }^{11}$ Version 5.05 [106, 113. Das Programm wurde von Wolfgang Eckstein vom Max-Planck-Institut für Plasmaphysik (IPP) in Garching entwickelt. SDTrimSP basiert auf der Methode der binären Kollisionen BCM und kombiniert das statische Monte-Carlo-Simulationsprogramm TRIM mit seiner dynamischen Version TRIDYN und konzertiert sich auf niederenergetische Kollisionen und Sputterprozesse. Das Target wird in der Simulation als eindimensional und amorph betrachtet. SDTrimSP bietet im Vergleich zu TRIDYN eine Vielfalt von Input-Möglichkeiten, einschließlich der Wahl der Wechselwirkungspotentiale und der unterschiedlichen Integrationsmethoden vom Abschirmintegral. Außerdem produziert SDTrimSP eine Vielzahl von Output-Daten mit allen möglichen Details von gesputterten Atomen [67]. Die Software wurde von Hans Hofsäss erweitert, um alle Momente der Erosions-, Redistributions- und Implantationskraterfunktion bis zur vierten Ordnung und bei Bedarf sogar Momente höherer Ordnung zu berechnen. Auf diese Weise ist es möglich, alle linearen und nichtlinearen Koeffizienten, die in den Arbeiten von Makeev et al. [95] und Bradley et al. [20] für Sigmund-Modell abgeleitet wurden, über Momente n-ter Ordnung von Kraterfunktionen zu berechnen. Eine andere Erweiterung von Hans Hofsäss ist die Berechnung von Driftgeschwindigkeitsprofilen der Rückstoßatome als Funktion der Tiefe. Denn die Driftgeschwindigkeit nahe der Oberfläche ist besonders wichtig, um den Dickenabhängigkeitskoeffizient $D_{11}$ genau zu berechnen. Mit dieser erweiterten Software steht nun ein universelles Simulationsprogramm zur Verfügung, mit dem die vollständigen Eingangsparameter für lineare und nichtlineare theoretische Modelle sowie für jede Ionenkombination und jede Ionenenergie bestimmt werden können [72].

In den Monte-Carlo-Simulationsprogrammen sind einige wichtige Parameter für die Berechnung des Sputteryields und Massentransports zu berücksichtigen. Zu den Parametern gehören die Oberflächenbindungsenergie $E_{S}$, die Volumenbindungsenergie $E_{b u l k}$, die Verlagerungsenergie $E_{D}$ und die so genannte cut-off-Energie $E_{\text {cut-off }}$, die ein Schwellwert für die Verfolgung der Atome in der Kollisionskaskade beschreibt. Die Oberflächenbindungsenergie $E_{S}$ stellt die planare Potentialbarriere für das Austreten der Atome aus dem Festkörper

\footnotetext{
${ }^{11}$ Static and Dynamic Transport of ions in matter Sequential and Parallel processing
} 


\section{Theorie der Musterbildung}

dar. $E_{s}$ ist abgeleitet von der Standardbildungsenthalpie des jeweiligen Elements und ist in SDTrimSP tabellarisiert. Die Volumenbindungsenergie $E_{b u l k}$ (engl.: bulk binding energy) ist die benötigte Energie, um eine Leerstelle in einer kristallinen Struktur zu schaffen. $E_{b u l k}$ ist nur in kristallinen Materialien definiert, denn in einem amorphen Target werden nur Atome umgeordnet und nur durch Erosion entfernt. Deswegen ist es zu empfehlen bei der Simulation von amorphen Targets die Volumenbindungsenergie gleich Null zu setzen. Die Verlagerungsenergie (engl.: displacement energy), die auch in kristallinen Materialien definiert und messbar ist, bestimmt die Rekombination von niederenergetischen rückgestoßonen Atomen mit ihren eigenen Leerstellen. Daher ist es sinnvoll für amorphe oder amorphisierbare Targets kleine oder sogar keine Verlagerungsenergie zu verwenden, da die Wahl von $E_{D}$ einen Einfluss auf den atomaren Massentransport innerhalb der Kollisionskaskade hat, denn für eine niederenergetische Kollisionskaskade berechnet mit großen $E_{D^{-}}$Werte werden die meisten erzeugten Rückstöße auf ihre ursprüngliche Position zurücksetzen, was im Durchschnitt zu einem nullwertigen Massentransport führt [67]. In einer vorherigen Studie haben wir die Musterbildung auf amorphem Kohlenstoff unter $\mathrm{Xe}^{+}$-Ionenbestrahlung untersucht [19]. In den ersten SDTrimSP-Berechnungen wurde eine Verlagerungsenergie $E_{D}=28 \mathrm{eV}$ für alle Ionenenergien gewählt, was zur Vernichtung der meisten Rückstoßereignisse insbesondere bei niedrigen Energien führt. Im Rahmen dieser Arbeit wurde die Volumenbindungsenergie $E_{B}$ sowie die Verlagerungsenergie $E_{D}$

für Ionenenergie $E_{\text {ion }}<5 \mathrm{keV}$ gleich Null gesetzt. Für Ionenenergie $E_{\text {ion }} \geq 5 \mathrm{keV}$ wurde eine Verlagerungsenergie $E_{D}=5-10 \mathrm{eV}$ gewählt. Die Wahl einer Verlagerungsenergie $E_{D}>0$ reduziert deutlich die Rechenzeit aber ohne Einfluss auf die Simulationsergebnisse [70, 71]. Das Simulationsprogramm SDTrimSP verwendet das Koordinatensystem $(x, y, z)$, wobei die $x$-Achse hier gegen die Oberflächennormale zeigt. Die $y$-Achse liegt in der Oberfläche entlang des projizierten Ionenstrahls. In dieser Arbeit wird das Koordinatensystem $(u, v, w)=(y,-z,-x)$ und $\left(u^{\prime}, v^{\prime}, w^{\prime}\right)$ für die lokale Oberfläche ähnlich wie in der BH-Theorie gewählt. Die Berechnung der Krümmungskeoffizienten benötigt eine genaue Definition der Oberfläche. Im SDTrimSP-Programm existieren die anfänglichen Positionen des Atomzentrums nur für $x \geq 0$. Die geometrische Oberfläche ist deshalb eine Ebene bei $x=0$, die nur zwischen Vakuum und Festkörper unterscheidet. Die Wechselwirkung zwischen Projektilen und Target-Atomen beginnt jedoch an einem Punkt $\left(-x_{C}, 0,0\right)$ über die geometrische Oberfläche (siehe Abbildung 2.15). Die Ebene $x=x_{C}$ definiert die Wechselwirkungsoberfläche und hängt vom maximal möglichen Stoßparameter ab. Ionen, die mit einem großen Winkel einfallen, können, bevor sie in das Target eintreten, über eine Distanz $x_{C} \cdot \tan \theta$ mit Target-Atomen wechelwirken. Für größere Ionenenergien, die ziemlich große Kollisionskaskaden erzeugen, ist der Unterschied zwischen der geometri- 
schen Oberfläche und der Wechselwirkungsoberfäche irrelevant. Aus diesem Grund ist die Wahl von $x_{C}$ vor allem bei niedrigen Ionenenergien von großer Bedeutung. Typischerweise beträgt $x_{C}$ etwa $0.3-0.5 \mathrm{~nm}$ über die geometrische Oberfläche. SDTrimSP verwendet strandardmäßig den Einschlagpunkt $\left(x_{C}, 0,0\right)$. Deswegen scheinen die rückgestoßenen und die gesputterten Atome zu positiven $y$-Werte durch $x_{C} \cdot \tan \theta$ verschoben zu werden. Diese Verschiebung ist signifikant bei niedriger Ionenenergie, wo die Größe der Kollisionskaskade im Vergleich zu $x_{C} \cdot \tan \theta$ klein ist [43, 70]. Die Simulationen wurden hauptsächlich im dynamischen Modus durchgeführt, um den Ionenimplantationseffekt zu berücksichtigen. Für jede Ionenenergie erfolgte die Simulation für 7 Oberflächenkrümmungen $K$ zwischen $K=-0.02 \mathrm{~nm}^{-1}$ und $K=+0.02 \mathrm{~nm}^{-1}$. Die Rückstöße werden berücksichtigt bis zu einer cut-off-Energie von $0,2 \mathrm{eV}$.

(a) BH coordinate systems

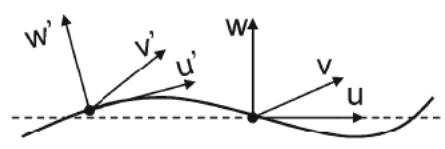

(b) SDTtrimSP coordinate system

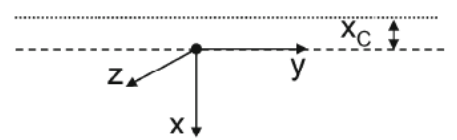

Abb. 2.15.: (a) Das übliche Koordinatensystem, das in Musterbildungsmodellen verwendet wird. (b) Das Koordinatensystem, das in den Monte-CarloSimulationen verwendet wird. Die gestrichelte Linie stellt die geometrische Oberfläche dar $(x=w=0)$, wo Atome nur für $x \geq 0$ oder $w \leq 0$ existieren. Die gepunktete Linie verweist auf die Wechselwirkungsoberfläche bei $x=-x_{C}[70]$

\subsubsection{Berechnung der Erosionskraterfunktion}

Die Berechnung des ersten Moments der Erosionskraterfunktion in Gleichung 2.32) und (2.34) erfolgt ähnlich zu Gleichung (47) von Ref. [118].

$$
M_{\text {eros. }}^{(1)}(\theta)=-\frac{Y(\theta)}{n} \cdot \delta_{u, \text { eros. }}(\theta)
$$

wobei $Y(\theta)$ der winkelabhängige Sputteryield ist, $n$ ist die Dichte der Target-Atome. Der Parameter $\delta_{u \text {,eros. }}(\theta)$ repräsentiert die mittlere Position der gesputterten Atome zum 
Einschlagpunkt und ist gegeben als:

$$
\delta_{u, \text { eros. }}(\theta)=\frac{1}{Z_{s p}} \sum_{i=1}^{Z_{s p}} y_{i, \text { start }}^{\text {erosion }}(\theta)
$$

Hier ist $Z_{s p}=Y \cdot N_{i o n}$ die Anzahl der gesputterten Atome und kann aus der Datei „output.dat" extrahiert werden. Die Anzahl der einfallenden Ionen $N_{\text {ion }}$ liegt zwischen $10^{17}$ und $10^{18}$ Ionen $/ \mathrm{cm}^{2}$ und ist so ausgewählt, dass sie mit den für die Bestrahlung verwendeten Flunzen übereinstimmt. $y_{i, s t a r t}$ sind die Anfangspositionen der gesputterten Atome und sind in der Datei „partic_back_r" aufgelistet.

\subsubsection{Berechnung der Redistributionskraterfunktion}

Die Berechnung des ersten Moments der Redistributionskraterfunktion erfolgt ähnlich zu Gleichung (49) von Ref. [118].

$$
M_{\text {redist. }}^{(1)}(\theta)=\frac{\delta_{u, \text { redist. }}(\theta)}{n}
$$

$\delta_{u, \text { redist. }}$ ist der zum projizierten Ionenstrahl parallele Massentransport pro Ion und ist definiert als:

$$
\delta_{u, \text { redist. }}(\theta)=N_{\text {knock }}(\theta) \cdot \frac{1}{Z_{R}} \sum_{i=1}^{Z_{R}}\left(y_{i, \text { end }}^{\text {recoil }}(\theta)-y_{i, \text { start }}^{\text {recoil }}(\theta)\right)
$$

$Z_{R}=N_{i o n} \cdot N_{D}$ stellt die gesamte Zahl der rückgestoßenen Atome mit Energie $>E_{D}$ dar, $N_{D}$ ist die Zahl der versetzten Atome pro Ion und $N_{\text {knock }}$ ist die Zahl der knock-onKollisionen. $y_{i, s t a r t}$ und $y_{i, \text { end }}$ sind die Anfangs- und Endpositionen der Rückstöße und sind in der Datei „partic_stop_r" aufgelistet. $N_{D}$ und $N_{k n o c k}$ können aus der Datei „output.dat" extrahiert werden. Der nach innen gerichtete Massentransport ist in Analogie $\mathrm{zu} 2.89$ dadurch gegeben:

$$
-\delta_{w, \text { redist. }}(\theta)=N_{\text {knock }}(\theta) \cdot \frac{1}{Z_{R}} \sum_{i=1}^{Z_{R}}\left(x_{i, \text { end }}^{\text {recoil }}(\theta)-x_{i, \text { start }}^{\text {recoil }}(\theta)\right) .
$$

Im Fall einer hochenergetischen Ionen mit $E_{\text {ion }} \geq 5 \mathrm{keV}$ und $E_{D}>0$, wird in den Berechnungen $N_{D}$ statt $N_{k n o c k}$ verwendet. 


\subsubsection{Berechnung der Dickenabhängigkeit $D_{11}$}

Die Dickenabhängigkeit ist laut Gleichung (2.63) gegeben zu:

$$
D_{11}=-v_{u}(h) \frac{\partial d}{\partial \theta}=-\frac{J}{n} \frac{\cos \theta}{d_{\text {top }}} \cdot f \sum_{i=1}^{N_{R, \text { top }}}\left(x_{i, \text { end }}^{\text {recoil }}-x_{i, \text { start }}^{\text {recoil }}\right) \cdot \frac{\partial d}{\partial \theta} .
$$

Der Term $v_{u}(h)$ ist die Driftgeschwindigkeit von rückgestoßenen Atomen in einer Oberflächenschicht mit der Dicke $d_{\text {top }}$ und $f=N_{R, t o p} / N_{D}$ ist der Anteil der Rückstoß-Ereignisse pro Ion in der obersten Oberflächenschicht $d_{t o r}{ }^{12}$ im Vergleich zur gesamten Rücktöße pro Ion $N_{D}$. Die gesamte Dicke der bestrahlten Oberflächenschicht $d$ wird berechnet als zweimal den Wert der mittleren Tiefe der Rückstoß-Atome $D$, wie bereits im Abschnitt (2.6.2) erwähnt wurde. $D$ kann als:

$$
D(\theta)=\frac{1}{N_{R}} \sum_{i=1}^{N_{R}} x_{i, \text { end }}^{\text {recoil }}(\theta)
$$

berechnet werden. $x_{i, s t a r t}$ und $x_{i, \text { end }}$ sind die Anfangs- und Endpositionen der Rückstöße in $x$-Richtung und sind in der Datei ,partic_stop_r" aufgelistet.

\subsubsection{Berechnung der Implantationskraterfunktion}

Die Berechnung des nullten und ersten Moments der Implantationskraterfunktion in Gleichung 2.69), 2.71) und 2.74 erfolgt durch die Beziehung

$$
M_{y, \text { implantation }}^{(n)}=\frac{\Omega}{N_{\text {ion }}} \sum_{i=1}^{N_{\text {impl. }}}\left(y_{i, \text { end }}^{\text {impl. }}\right)^{n} .
$$

Die Endpositionen der implantierten Ionen können aus der Datei „partic_stop_p.dat“ extrahiert werden. Die mittlere Endposition der implantierten Ionen ist:

$$
\left\langle y_{\text {end }}^{\text {impl. }}\right\rangle=\frac{1}{N_{\text {impl. }}} \sum_{i=1}^{N_{i m p l .}} y_{i, \text { end }}^{i m p l}
$$

\footnotetext{
${ }^{12} \mathrm{Im}$ Rahmen dieser Arbeit wurde $d_{t o p}$ als die Dichte der ersten drei atomaren Monolagen gewählt.
} 
Der nullte und erste Moment der Implantationskraterfunktion kann mit Hilfe von Gleichung 2.93) in folgender Weise berechnet werden:

$$
\begin{gathered}
M_{y, i m p l .}^{(0)}=\Omega \cdot f_{i m p l .} \\
M_{y, i m p l .}^{(1)}=\Omega \cdot f_{i m p l .}\left\langle y_{\text {end }}^{i m p l .}\right\rangle,
\end{gathered}
$$

wobei $f_{\text {impl. }}=N_{\text {impl. }} / N_{\text {ion }}$ die Zahl der implantierten Ionen im Vergleich zur Gesamtzahl der einfallenden Ionen ist [75].

\subsubsection{Bestimmung des Koeffizients für ioneninduziertes viskoses Fließen}

Der Koeffizient für das ionenstrahlinduzierte viskose Fließen in der Dünnschicht-Annäherung [70] ist in Gleichung (2.44) und 2.45 durch

$$
F_{S, r a d} \cdot d^{3}=\frac{\gamma \cdot J \cdot \cos \theta}{3 \eta_{S, r a d}} \cdot d^{3}=\frac{\gamma \cdot J}{3 p_{0} \cdot n} \cdot \cos \theta \cdot N_{D} \cdot d^{2}
$$

gegeben. $d=2 D$ ist die gesamte Dicke der bestrahlten Schicht. $\eta_{S, \text { rad }}$ ist die material spezifische ioneninduzierte Viskosität [141]. Der Parameter $\mathrm{p}_{0}($ Einheit $\mathrm{Pa} \cdot \mathrm{dpa}){ }^{13}$ ist ein Skalierungsfaktor, eingeführt von Mayr et al. [99, 148, um die inonenstrahlinduzierten Viskosität für verschiedene Energien und Einfallswinkel skalieren zu können. Die ionenstrahlinduzierte Viskosität $\eta_{S, r a d}$ wird nach dem von Mayr et al. eingeführten Referenzfaktor $\mathrm{p}_{0}$ skaliert. Die Werte von $\mathrm{p}_{0}$ bei einem Einfallswinkel von $65^{\circ}$ werden als Referenz verwendet, um die Viskosität für andere Winkel zu skalieren. Die typische Viskosität liegt für Silizium bei $\eta_{S, \text { rad }}=1-5 \cdot 10^{23} \mathrm{~Pa} \cdot$ Ionen $/ \mathrm{cm}^{2}$ und für Kohlenstoff bei $\eta_{S \text {, rad }}=1-3 \cdot 10^{24}$ $\mathrm{Pa} \cdot$ Ionen $/ \mathrm{cm}^{2}$. Eine genaue Bestimmung der ionenstrahlinduzierten Viskosität kann erreicht werden (sobald experimentelle Daten für die Rippel-Wellenlänge vorhanden sind), indem man den Skalierungsfaktor $\mathrm{p}_{0}$ mit Hilfe Gleichung (2.83) variiert, bis eine ähnliche Wellenlänge mit maximaler Wachstumsrate reproduziert werden kann [65]. In Abbildung (2.16) ist einen Modellfall für $500 \mathrm{eV} \mathrm{Ne}^{+}$Ionen, die auf eine amorphe Kohlenstoff-Schicht einfallen. Die Abbildung zeigt die unterschiedlichen Krümmungskoeffizienten zu $C_{11}$ (Gl.

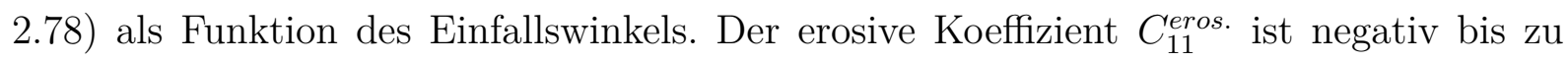

\footnotetext{
${ }^{13} \mathrm{~Pa} \cdot \mathrm{dpa}=$ Pascal $\cdot$ displacements per atom
} 
etwa $65^{\circ}$ und wird dann stark positiv. Dadurch trägt $C_{11}^{\text {eros. }}$ ab $65^{\circ}$ zu einer Stabilisierung der Oberfläche bei. Der redistributive Koeffizient $C_{11}^{\text {redis. }}$ zeigt das vom CV-Modell vorhergesagte Verhalten und ist auch in Übereinstimmung mit anderen Simulationen, die der CFF verwendet haben [78, 79, 82, 119]. Die dynamische Dickenabhängigkeit $D_{11}$ ist immer positiv mit einem Maximum bei etwa $50^{\circ}$ und hat somit einen deutlichen stabilisierenden Beitrag. Die erosive Krümmungsabhängigkeit $T_{11}$ ist leicht positiv bis zu $67^{\circ}$, dann trägt sie stark zu einer Stabilisierung der Oberfläche bei. Schließlich ergibt sich der totale Krümmungskoeffizient $C_{11}^{\text {total }}$ aus der Summe von allen Beiträgen wie in Abbildung (2.16) dargestellt ist. Für den Winkelbereich zwischen $50^{\circ}$ und $85^{\circ}$ ist $C_{11}^{\text {total }}<0$ und eine Gesamt-Oberflächeninstabilität kann zur Bildung von parallelen Rippel-Mustern in $u$-Richtung führen. Dieses Bereich wird in Abbildung (2.16) als gelb beschattete Fläche angezeigt.

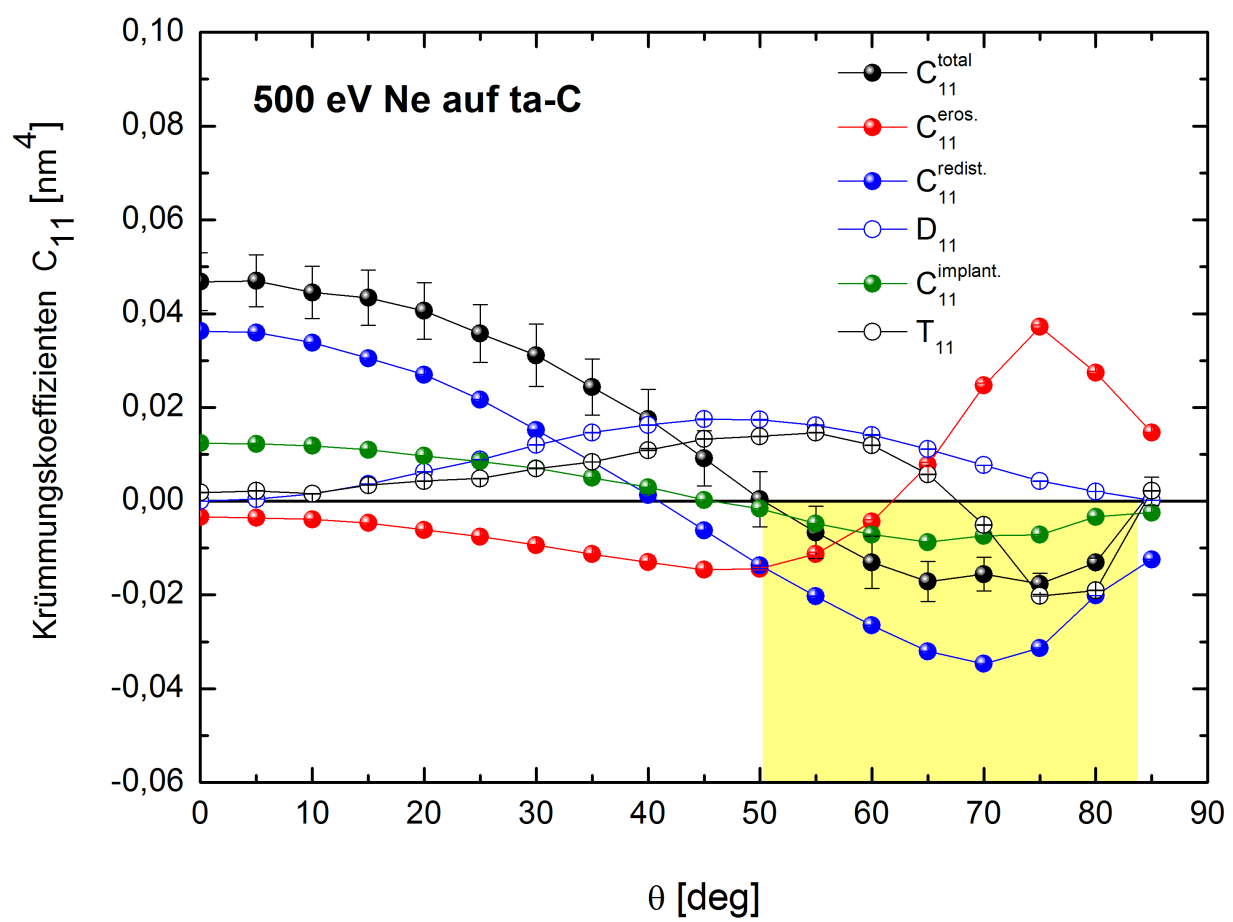

Abb. 2.16.: Zusammenstellung der Krümmungskoeffizienten, die zu $C_{11}$ beitragen, für den Fall 500 eV Ne${ }^{+}$auf amorphe Kohlenstoffschicht. 


\subsection{Surfactant Sputtering}

Das Surfactant ${ }^{14}$ Sputtering ist eine neuartige und vielseitige Technik, die eine stationäre Oberflächenbedeckung mit geeigneten Selbst- oder Fremdatomen (Surfactant-Atome) gleichzeitig während der Ionenstrahl-Erosion verwendet (Abbildung 2.17). SurfactantAtome verursachen eine Reduzierung des Substrat-Sputteryields und außerdem können abhängig von der Depositionsrate und von physikalischen und chemischen Eigenschaften von Substrat und deponierter Substanz zu einer Vielzahl von neuen Oberflächenmorphologien führen [68, 74, 153, 157]. Beim senkrechten Ioneneinfall sagt das CV-Modell, in Übereinstimmung mit experimentellen Befunden, eine gleichmäßige glatte Oberfläche vorher [28, 155]. Allerdings führt eine simultane Zufuhr von bestimmten metallischen Surfactant-Atomen zu einer ausgeprägten Musterbildung. Mehrere theoretische Studien haben die Kodeposition von Surfactant-Atomen während der Sputtererosion als wesentlichen Parameter für die Musterbildung betrachtet. Kree et al. haben zunächst eine Kontinuumstheorie sowie eine Monte-Carlo Simulationen unter Berücksichtigung der Kodeposition von Surfactant-Atomen vorgestellt [62, 85]. Die Monte-Carlo Simulationen basieren auf dem solid-on-solid-Modell mit zwei Arten von Atomen A und B. Das Kontinuumsmodell berücksichtigt Surfactants durch unterschiedliche Oberflächendichte, unterschiedliche Difussionsstromdichte und individuellen Sputteryields in Abhängigkeit von der lokalen Oberflächendichte. Je nach Verhalten des A- und B-Atoms können Zusammensetzungsmodulationen von Surfactants, Oberflächenglättungen oder neuartige Musterbildung wie z.B. Punktmuster auftreten. In einer anderen theoretischen Studie hat M. Bradley die Ionenstrahlerosion unter simultaner Zufuhr von surfactant-Atomen betrachtet und ein Modell für Musterbildung, das kein krümmungsabhängiges Sputtern oder CV-Effekt erfordert, eingeführt [21. Das Modell berücksichtigt Konzentration und lokale winkelabhängige Erosionsraten zweier Komponenten A (Surfactant) und B (Substrat) sowie die durch viskoses Fließen induzierten Ströme. Thermische Diffusion und chemische Wechselwirkungen werden in diesem Modell vernachlässigt. Beim senkrechten Ioneneinfall mit simultanen Kodeposition von Surfactant-Atomen erwartet das Bradley-Modell eine instabilen Oberfläche und somit eine Musterbildung, wenn der Sputteryield des Substrats bei einer senkrechten Ionenbestrahlung höher als der Sputteryield einer Kodeponierten Spezies ist. In anderen Worten, wenn das Substrat vorzugsweise gesputtert wird. Allerdings ist der Sputteryield des Substrats in den meisten experimentellen Daten zur Sputtererosion unter simultaner Kodeposition viel kleiner als der Sputteryield einer kodeponierten Spezies [74, 153, 157]. Hofsäss et al. [68] haben ein Musterbildungs-Szenario basierend auf

\footnotetext{
${ }^{14}$ surface active agents
} 
lonenstrahl
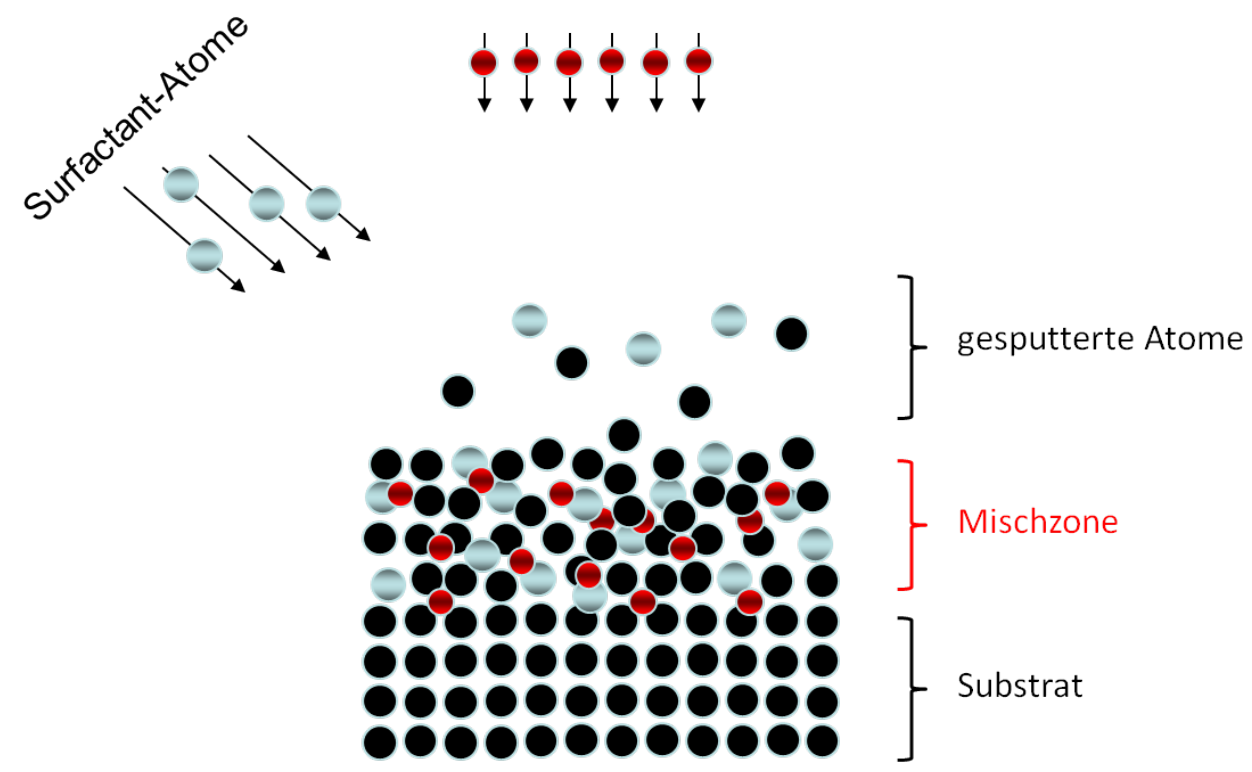

Abb. 2.17.: Schematische Darstellung der Sufactant-Sputtern-Prinzip. Ein homogener breiter Ionenstrahl trifft auf das Substrat unter simultaner Kodeposition von Surfactant-Atomen.

anfänglichen chemischen Effekten und Phasenseparationsprozesse eingeführt. Sie untersuchten die Musterbildung auf Silizium mit Schrägeinfall-Metall-Kodeposition und senkrechten Edelgas-Ionenbestrahlung und konnten experimentelle Hinweise für die Bildung einer amorphen Metallsilizidschicht während des Surfactant-Sputterns liefern. Die MetallTargets für die Kodeposition waren hochreine Folien, die neben dem Siliziumsubstrat mit einem Neigungswinkel von $30^{\circ}$ positioniert waren. Die Metall-Targets (Fe, Ni, Mo, W, Pt, und $\mathrm{Au}$ ) wurden entsprechend ihrer Fähigkeit, Metallsilizide zu bilden, ausgewählt. Die ausgeprägteste Neigung zur Musterbildung besteht für die Metalle Fe, Ni, Mo, und W, die Si-reich amorphe Silizide mit Stöchiometrie $\mathrm{MeSi}_{2}$ bilden (Abbildung 2.18). Eine mittlere stationäre Metallkonzentration in der Oberflächenschicht von 3 at.\% ist ausreichend, um eine ioneninduzierte Musterbildung auszulösen. Metall-Surfactants, für die nur metallreiche Silizide existieren oder bei denen keine stabile Silizidphase existiert, erzeugen keine oder nur schwache Muster auf Siliziumoberflächen [68, 157]. Die vorgeschlagene Phasenseparation und Silizidbildung von Hofsäss et al. wurde von anderen Gruppen untersucht und bestätigt [44]. Basierend auf diesen Ergebnissen und auf den binären Phasendiagrammen haben Hofsäss et al. eine ausgeprägte ioneninduzierte Musterbildung auf Kohlenstoffschichten mit Metall-Kodeposition vorhergesagt [68]. Im Rahmen dieser Arbeit wurden die Musterbildung auf amorphe Kohlenstoffschichten bei senkrechtem Ioneneinfall und simultanen Kodeposition von Mo-, W-, Ti-, und Pt-Atomen untersucht, um die chemi- 
schen Effekte auf die Musterbildung, insbesondere die Tendenz zur Phasenseparation zu bestätigen und zu verallgemeinern.

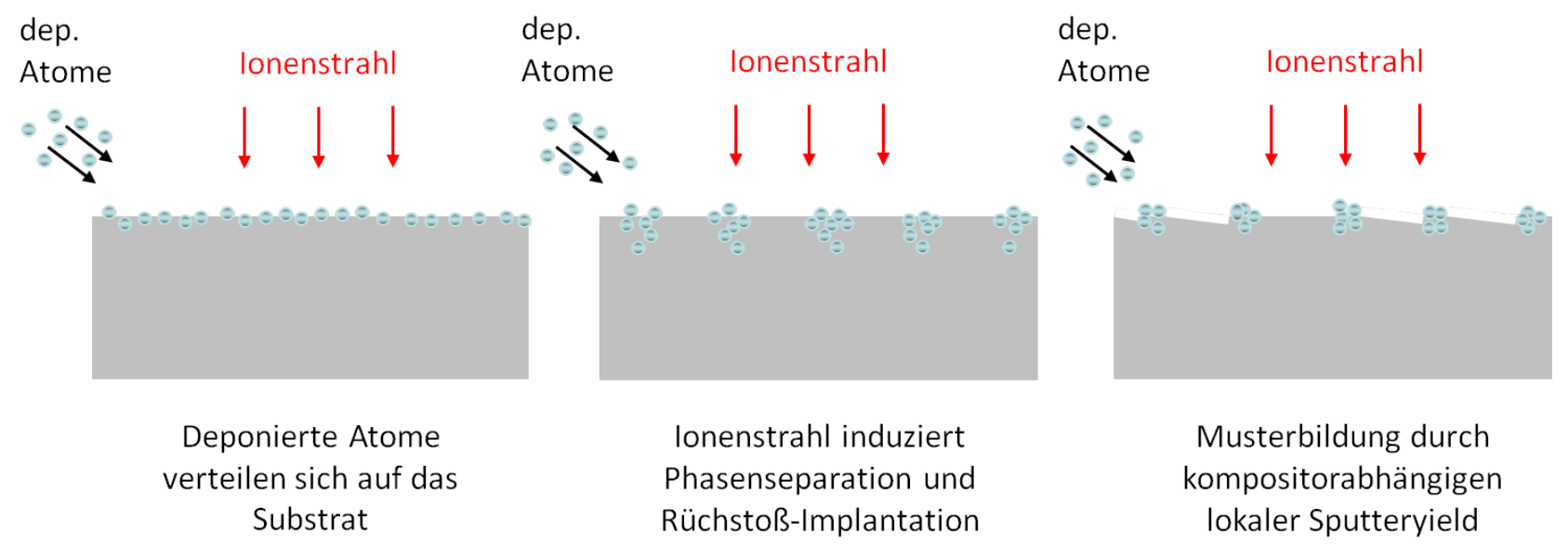

Abb. 2.18.: Szenario für die Musterbildung auf Si-Oberflächen während der Kodeposition von Fe-, Ni-, Mo-, und W-Atome. 


\section{Methoden}

\subsection{Probenpräparation}

In dieser Arbeit wird die Oberflächenstrukturierung von Siliziumoberflächen in (100)Orientierung sowie diamantähnliche tetraedrische wasserstofffreie amorphe Kohlenstoffschichten (ta-C) untersucht. Die ta-C Schichten bestehen überwiegend aus sp ${ }^{3}$-hybridisierten Bindung und wurden mit Hilfe der Laser-Arc-Technologie am Fraunhofer-Institut für Werkstoff- und Stahltechnik (IWS) in Dresden hergestellt. Dabei wird eine gepulste Vakuumbogenentladung jeweils durch einen kurzen Laserpuls an einer definierten Stelle einer Kathoden-Walze aus Graphit gezündet und somit ein Plasma aus durchweg ionisierten hochenergetischen Kohlenstoffteilchen erzeugt. Durch Überlagerung der linearen Verschiebung des Laserfokus mit der Kathodenrotation wird sowohl eine homogene Beschichtung über die gesamte Höhe als auch ein gleichmäßiger Abtrag der Kathoden-Walze ermöglicht [3. Die Schichtdicke beträgt ca. $270 \mathrm{~nm}$ und die Schichten sind extrem glatt mit rms-Rauigkeit von etwa $0.1 \mathrm{~nm}$.

\subsection{Ionenbestrahlung}

Die Bestrahlung der Proben mit Ionen im Energiebereich zwischen $100 \mathrm{eV}$ und $10 \mathrm{keV}$ erfolgte an drei Anlagen, einer Plasmaquelle sowie dem Ionenbeschleuniger IOSCHKA und dem Ionenbeschleuniger ADONIS. In diesem Abschnitt werden die drei Anlagen detailliert erläutert.

\subsubsection{Die Plasmaquelle}

Im Rahmen dieser Arbeit wurde die niederenergetische Plasmaquelle (Gen II) der Firma Tectra GmbH für Bestrahlungen mit Ionen mit Energien von bis zu $2 \mathrm{keV}$ verwendet. Die Quelle wurde von Hayo Zutz im II. Physikalischen Institut in Göttingen aufgebaut und besteht aus einem wassergekühlten Mikrowellenmagnetron mit einer Frequenz von 4.45 GHz, zwei elektrostatischen Gittern und zwei Netzteilen (Abbildung 3.1). 


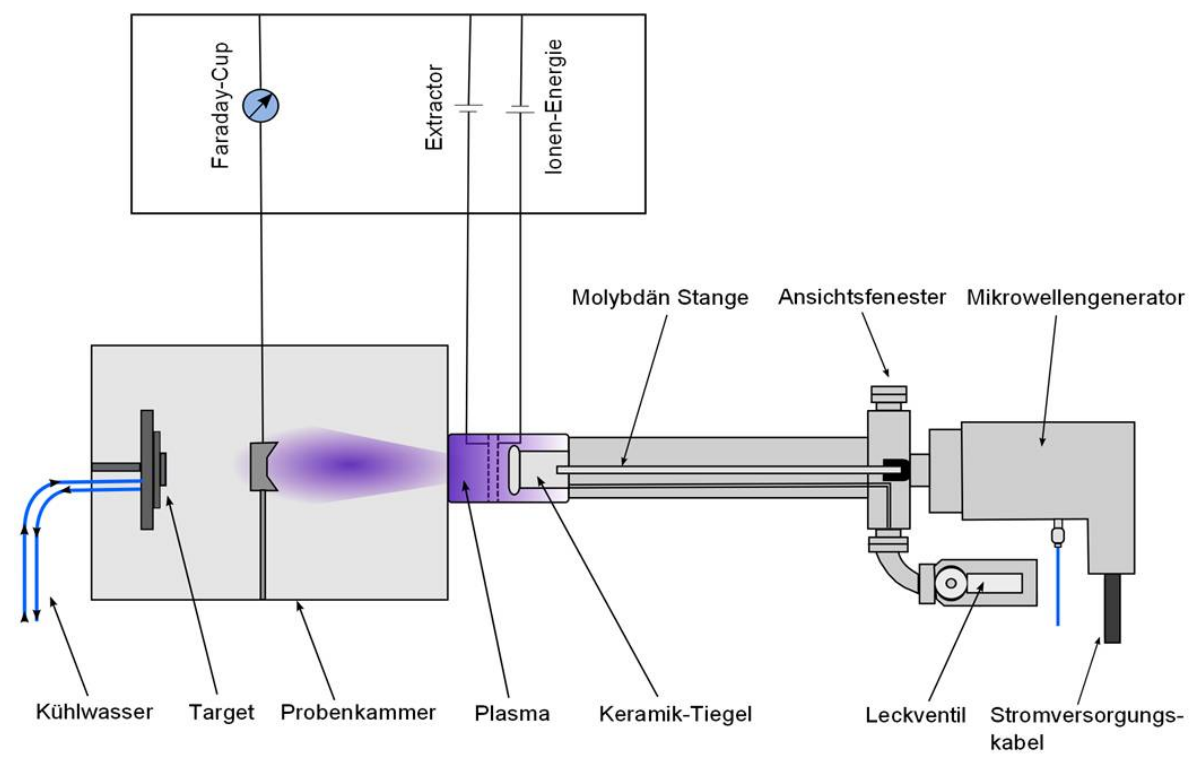

Abb. 3.1.: Schematischer Aufbau der Plasmakammer.

Das Plasma wird von rechts (in Abb. 3.1) mit Mikrowellenenergie versorgt. Aus dem Magnetron kommend wird die Mikrowellenleistung durch die Molybdän-Stange in den Keramiktiegel geführt. Das in den Tiegel zugeführte Gas wird dadurch ionisiert und so ist das Plasma gezündet. Bei Anlegen einer positiven Spannung an das erste Gitter und negativer Spannung an das Extraktionsgitter können die im Plasma befindlichen Ionen auf eine kinetische Energie von bis zu $2 \mathrm{keV}$ beschleunigt werden. Aufgrund der hohen Leistung wird das Target mit Wasser gekühlt. Für einen optimalen Ionenstrahl muss der Extraktionsstrom auf das Extraktionsgitter durch Variation der Extraktionsspannung minimiert werden. Die Plasmaquelle kann mit unterschiedlichen Gasen betrieben werden, beispielsweise Luft, Ar, Xe, $\mathrm{N}_{2}, \mathrm{O}_{2}$, oder He (Abbildung 3.2). Der Betriebsdruck für die meisten Gase beträgt ca. $10^{-3}$ mbar.
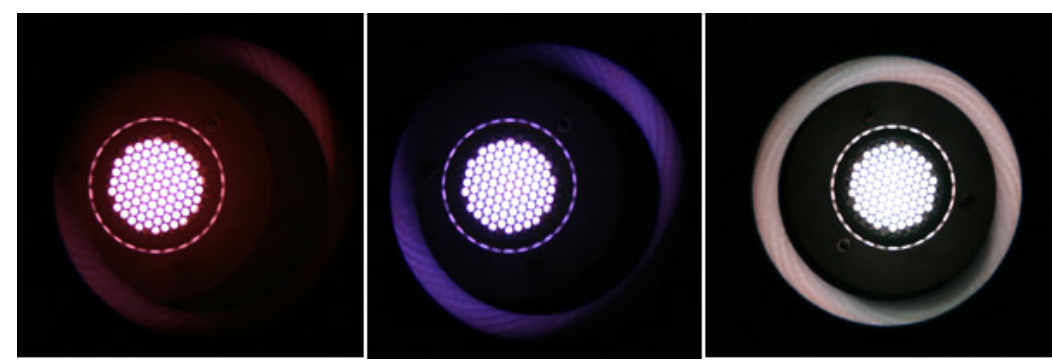

Abb. 3.2.: Unterschiedliche Gas-Plasma erzeugt an der Anlage (Tectra Gen II). Rot für Luft, Violett für Argon und Hellblau für Helium [5] 
Das Helium-Plasma kann jedoch aufgrund der hohen Ionisierungsenergie von Helium $\left(\mathrm{E}_{\text {ion }}=24.5 \mathrm{eV}\right)$ nur bei hohem Druck (ca. $10^{-2}$ mbar) generiert werden. Die Anlage kann ebenfalls als Atomquelle betrieben werden, so werden die Ionen und Elektronen mittels eines elektrostatischen Gitters neutralisiert bzw. herausgefiltert. Dazu wird eine Ionenfalle und ein zusätzliches Netzteil benötigt [17].

\subsubsection{Ionenbescheuniger IOSCHKA}

Der Ionenbeschleuniger IOSCHKA (IOnen SCHleuder KAmmer) ist ein Niederenergiebeschleuniger für Energien bis zu $10 \mathrm{keV}$. Bei der Ionenquelle handelt es sich um eine Colutron-Ionenquelle [1]. Aus dem heißen Filament in der Strahlführungskammer werden Elektronen emittiert und durch die Anodenspannung beschleunigt (Abbildung 3.3). Durch die beschleunigten Elektronen werden durch Stöße die Gasatome ionisiert und diese wiederum mit einer Kombination von drei elektrostatischen Linsen beschleunigt und fokussiert. Mit einem Wien'schen Geschwindigkeitsfilter werden die Ionen massensepariert. In der Implantationskammer wird der Ionenstrahl durch einen sogenannten Beamsweep, der eine gleichmäßige Bestrahlung von Probenoberflächen ermöglicht, periodisch abgelenkt. Nach dem Beamsweep folgt eine Blende mit einem Durchmesser von $11 \mathrm{~mm}$, um den noch inhomogenen Ionenstrahl abzuschneiden. Durch Anlegen einer Gegenspannung an das Target werden die Ionen auf die benötigte Energie abgebremst. Der Strahl wird anschließend mit einer Suppressorlinse auf die Probe fokussiert. Es wird ein Kreis mit einem Durchmesser von $12 \mathrm{~mm}$ bestrahlt.

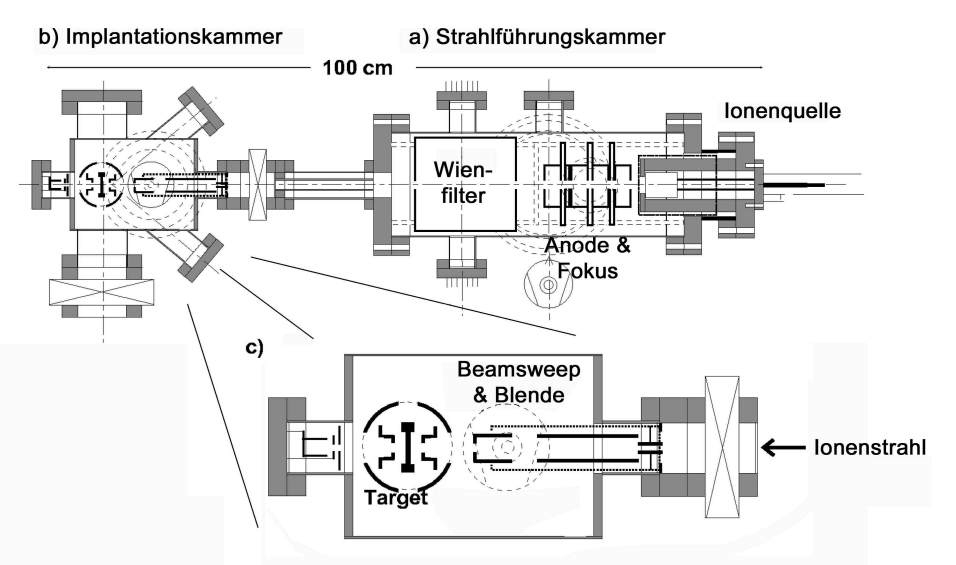

Abb. 3.3.: Schematische Darstellung des Ionenbeschleunigers IOSCHKA. a) Strahlungskammer mit Ionenquelle, b) Implantationskammer und c) Vergrößerung der Implantationskammer [60] 


\subsubsection{Ionenbeschleuniger ADONIS}

Bei der Ionenquelle von ADONIS1 handelt es sich um eine Hochtemperatur-SideniusIonenquelle. In der Quelle wird das zu ionisierende Gas, zum Beispiel Ar, $\mathrm{N}_{2}$ oder $\mathrm{CO}_{2}$, direkt eingeleitet und durch Stoßionisation ein Plasma erzeugt. Durch Anlegen einer Hochspannung von $30 \mathrm{kV}$ werden die Ionen extrahiert und beschleunigt. Zur Fokussierung des Ionenstrahls dient eine elektrostatische Linse (17-21 kV). Mittels eines 90 Sektormagneten werden die Ionen massensepariert und dann die jeweils gewünschte Ionenart ausgewählt. Nach dem Magneten folgt eine weitere Fokussierung des Ionenstrahls mittels einer elektrostatischen Linse (0-12 kV) sowie einer Quadrupollinse. Vor der Depositionskammer erfolgt durch einen Deflektor eine elektrostatische Ablenkung des Ionenstrahls um $5^{\circ}$, um die Neutralteilchen aus dem Strahl zu entfernen und somit mögliche Defekte in der deponierten Schicht zu vermeiden. Durch Anlegen einer Gegenspannung (0-30 kV) in der Depositionskammer werden die ankommenden Ionen auf die benötigte Energie abgebremst [24, 57, 64, 130, 135, 163].

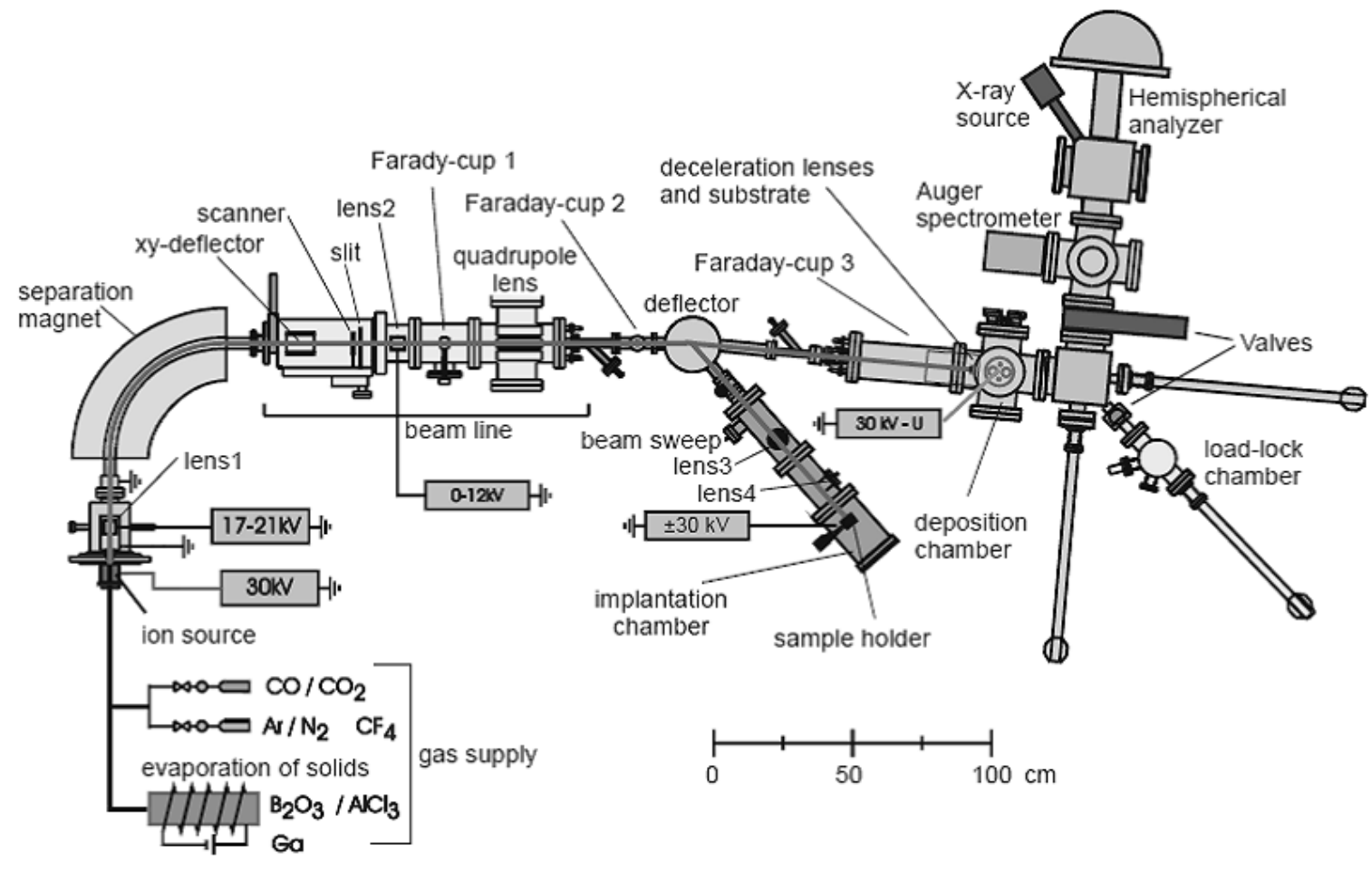

Abb. 3.4.: Schema des Ionenbeschleunigers ADONIS [57].

${ }^{1}$ Anlage zur DepOsition von Niederenergetischer Ionen auf Sustrate. 


\subsection{Mess- und Analyseverfahren}

\subsubsection{Rasterkraftmikroskop}

Das Rasterkraftmikroskop $\mathrm{AFM}^{2}$ ist ein Typ eines Rastersondenmikroskops und wird für die Untersuchung der Proben vor und nach der Bestrahlung verwendet. Durch eine sehr feine, lokal mit der Probenoberfläche wechselwirkende Spitze (Spitzenradius 7-50 nm) wird die Oberfläche in einem Rasterverfahren Zeile für Zeile abgetastet. Die feine Spitze befindet sich an einer Balkenfeder (Cantilever), die durch die Wechselwirkung zwischen Spitze und Probe ausgelenkt wird. Die Verbiegung der Balkenfeder wird durch Ablenkungen eines auf die Spitze gerichteten Laserstrahls mit Hilfe einer Photodiode detektiert (Abbildung 3.5). Um die Spitze in alle drei Raumrichtungen bewegen zu können, verwendet man im Rasterkraftmikroskop ein Piezoröhrchen, das außen mit vier Metallelektroden und einer Ringelektrode auf der Innenseite versehen ist. Zur Verbiegung des Piezoröhrchens in eine bestimmte $x$ - $y$-Richtung legt man eine Spannung an zwei gegenüberliegende Elektroden an. Für eine Verschiebung in $z$-Richtung ist eine Spannung sowohl an der Ringelektrode als auch an den vier Außenelektroden erforderlich.

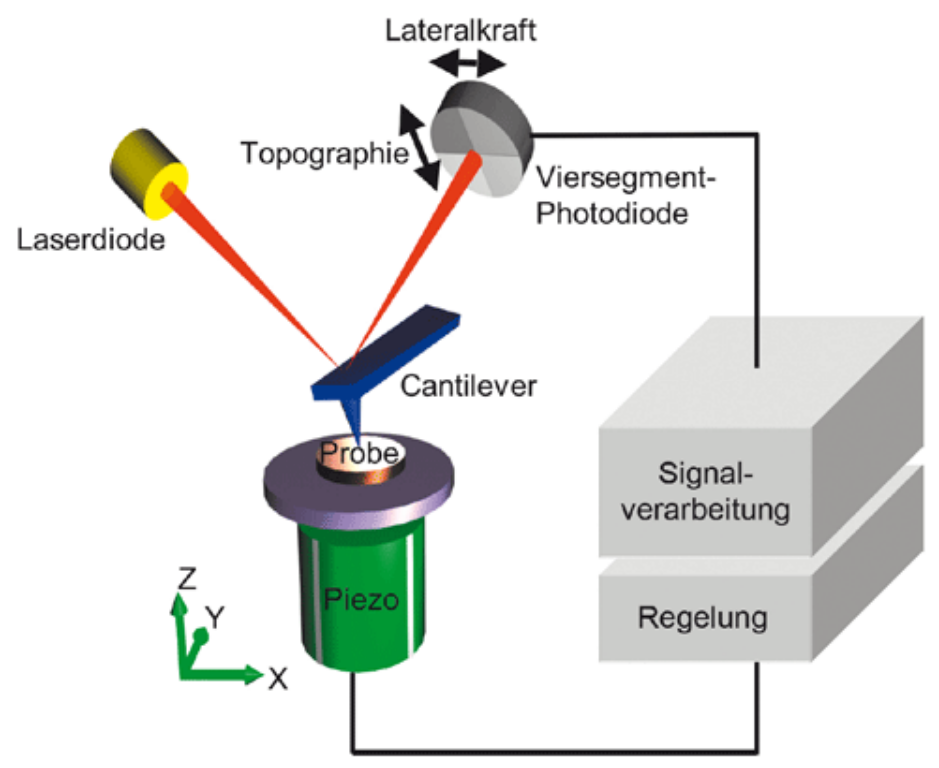

Abb. 3.5.: Funktionsprinzip eines Rasterkraftmikroskop [4].

Die Funktion des Rasterkraftmikroskop beruht auf der Kraftwechselwirkung zwischen Spitze und Oberfläche. Es handelt sich hier um attraktive Kräfte wie kovalente, metallische oder ionische Bindungen oder Van-der-Waals-Kräfte sowie repulsive Kräfte wie Pauli- und

\footnotetext{
${ }^{2}$ Atomic $\boldsymbol{F}$ orce Microscope.
} 


\section{Methoden}

Coulombabstoßung. Zusammengenommen können die verschiedenen Wechselwirkungen mit dem Lennard-Jones-Potential

$$
V(r)=\left(\frac{c_{1}}{r^{12}}-\frac{c_{2}}{r^{6}}\right)
$$

beschrieben werden. Hierbei ist $r$ der Abstand zwischen Spitze und Probe. Der erste Term beschreibt die repulsive Wechselwirkung, der zweite Term die attraktive Wechselwirkung. Das Verhältnis zwischen attraktivem und repulsivem Potential wird durch die Konstanten $c_{1}$ und $c_{2}$ beschrieben. Nähert man die Spitze und die Probe einander an, erfährt die Spitze eine anziehende Kraft. Bei weiterer Annährung werden sich die Elektronenorbitale der Atome auf der Spitze mit denen der Probe überlappen. Nach dem Pauli-Prinzip erfahren Spitze und Probe dann eine starke abstoßende Kraft (Abbildung 3.6). Beim AFM unterscheidet man zwischen verschiedenen Betriebsmodi. Grundsätzlich unterteilen sich die AFM-Betriebsmodi nach vier wichtigen Arten. Zum einen in Kontakt- und Nichtkontakt-Betriebsarten und zum anderen in statische und dynamische Betriebsarten. Die Kontaktbetriebsarten sind durch einen mechanischen Kontakt zwischen Spitze und Probe gekennzeichnet, während bei Nichtkontaktbetriebsarten Spitze und Probe zwar sehr dicht angenährt werden, sich aber nicht berühren. Bei dynamischen Betriebsarten wird die Balkenfeder vertikal moduliert und die Schwingungsamplituden sowie Frequenzen ausgewertet, während bei statischen Betriebsarten nur eine Verbiegung der Balkenfeder durch die Probe hervorgerufen wird.

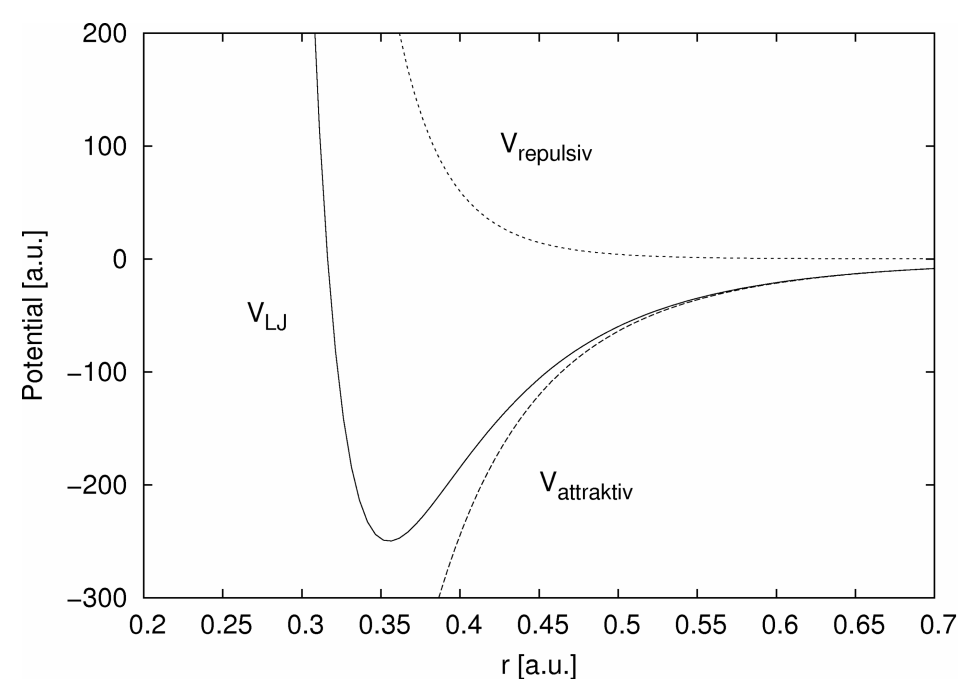

Abb. 3.6.: Verlauf des Lennard-Jones-Potentials für $c_{1} / c_{2}=10^{-3}$. Die Abbildung zeigt die Summe aus abstoßenden und anziehenden Kräften. [25].

Der in dieser Arbeit verwendete Betriebsmodus war der statische Kontaktmodus. Hier- 
bei wird die Spitze im Kontakt über die Probe gerastert und der Abstand zwischen Probe und Spitze jeweils so reguliert, dass die Durchbiegung der Balkenfeder konstant bleibt [87]. Auf dieser Weise wird ein Bild der Oberfläche bei konstanter Auflagekraft der Spitze aufgenommen, was wesentlich die Topographie der Probenoberfläche widerspiegelt. Über die Durchbiegung der Balkenfeder kann die Auflagekraft eingestellt werden. Typische Kräfte liegen hierbei im Bereich von $\mathrm{nN}$.

\subsubsection{Rutherford-Rückstreu-Spektrometrie}

Die Rutherford-Rückstreu-Spektrometrie RBS ${ }^{3}$ ist eine Methode zur tiefenaufgelösten Materialanalyse. Hierbei wird ein masseselektierter Ionenstrahl auf die zu untersuchende Probe gerichtet. Die eindringenden Ionen mit der Masse $M_{P}$ werden teilweise durch elastische Stöße an den Kernen der Targetatome mit der Masse $M_{T}$ zurückgestreut und durch einen energieauflösenden Detektor registriert. Aufgrund des Energieverlustes der zurückgestreuten Teilchen können Rückschlüsse auf die Masse der Targetatome und die Tiefe der Streuereignisse gezogen werden (Abbildung 3.7). Bei Teilchenenergien, die für die Rutherford-Rückstreuung in Frage kommen, kann man von einer Coulomb-Streuung ausgehen. Durch Coulomb-Abstoßung werden einfallende Ionen wie bei einem elastischen Stoß an den Atomkernen des Targetmaterials gestreut. Der dabei auftretende Energieverlust der zurückgeworfenen Teilchen lässt sich durch den kinematischen Faktor $k$ beschreiben.

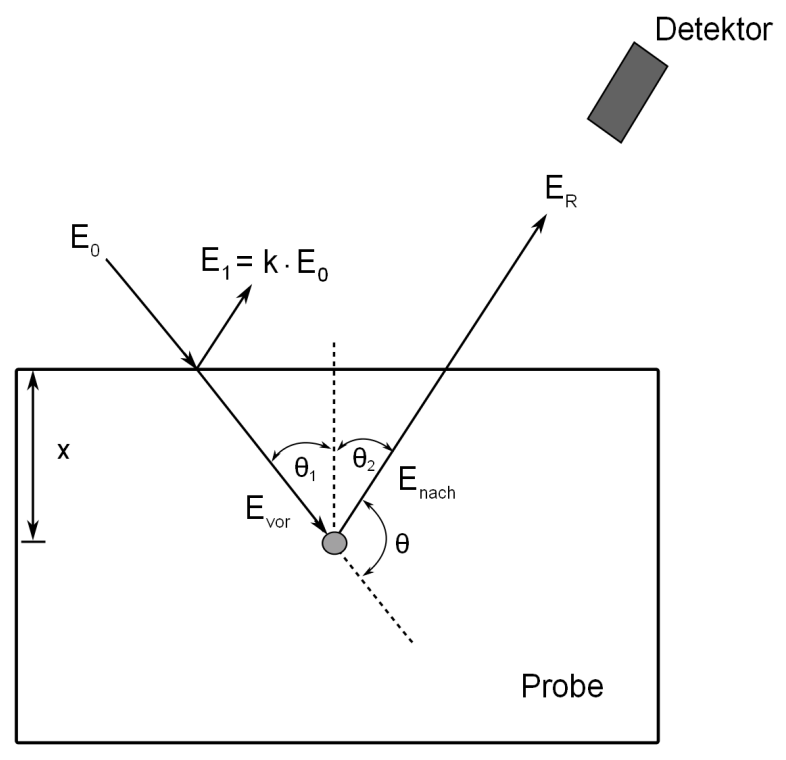

$\boldsymbol{A} \boldsymbol{b} \boldsymbol{b}$. 3.7.: Schematische Darstellung der Rückstreuung eines Ion in der Tiefe $x$.

\footnotetext{
${ }^{3} \boldsymbol{R}$ utherford Backscattering Spectrometry.
} 


\section{Methoden}

Der kinematische Faktor $k$ beschreibt den Enegieübertrag vom Projektil der Energie $E_{0}$ und Masse $M_{P}$ an das Targetteilchen der Masse $M_{T}$. Die Energie des gestreuten Teilchens ist mit

$$
E_{1}=k \cdot E_{0}
$$

gegeben, wobei aus der Energie- und Impulserhaltung die folgende Beziehung folgt:

$$
k=\left(\frac{\sqrt{1-\left[\left(M_{P} / M_{T}\right) \sin \theta\right]^{2}}+\left(M_{P} / M_{T}\right) \cos \theta}{1+\left(M_{P} / M_{T}\right)}\right)^{2}
$$

Der kinematische Faktor wird nur durch das Massenverhältnis $M_{P} / M_{T}$ und dem Streuwinkel $\theta$ bestimmt. Abdildung (3.8) zeigt die Abhähngigkeit des kinematischen Faktors vom Massenverhältnis $M_{P} / M_{T}$ und vom Streuwinkel $\theta$. Die gestreuten Ionen weisen in einen Streuwinkel nahe $180^{\circ}$ eine besonders gute Massentrennung auf. Deswegen wäre es am besten, für eine optimale Massentrennung, die Detektoren im Winkel nahe $180^{\circ}$ aufzustellen. Trifft ein Ion unter dem Winkel $\theta_{1}$ auf das Target, so wird das Ion beim Durchqueren von Materie Energie verlieren (Abbildung 3.7). Wird der Energieverlust pro Strecke bei D" $\breve{n n s c h i c h t e n ~ n a ̈ h e r u n g s w e i s e ~ k o n s t a n t ~ a n g e n o m m e n, ~ s o ~ b e t r a ̈ g t ~ d i e ~ E n e r g i e ~}$ des Ions kurz vor der Streuung

$$
E_{\mathrm{vor}}=E_{0}-\frac{x}{\cos \theta_{1}} \cdot\left(\frac{\mathrm{dE}}{\mathrm{dx}}\right)_{\text {Hin }} .
$$

Die Energie nach der Streuung wird durch den kinematischen Faktor bestimmt.

$$
E_{\text {nach }}=k \cdot E_{\mathrm{vor}} \text {. }
$$

Aufgrund des elektronischen Energieverlusts auf dem Rückweg ergibt sich die Energie des Ions $E_{R}$ beim Verlassen der Probe zu:

$$
E_{R}=E_{\text {nach }}-\frac{x}{\cos \theta_{2}} \cdot\left(\frac{\mathrm{dE}}{\mathrm{dx}}\right)_{R}
$$

Für die Energiedifferenz zwischen einem an der Oberfläche und einem in der Tiefe $x$ 
gestreuten Ion gilt:

$$
\Delta E=k \cdot E_{0}-E_{R}=\left[\frac{k}{\cos \theta_{1}} \cdot\left(\frac{\mathrm{d} E}{\mathrm{~d} x}\right)_{\mathrm{Hin}}+\frac{1}{\cos \theta_{2}} \cdot\left(\frac{\mathrm{d} E}{\mathrm{~d} x}\right)_{R}\right] \cdot x .
$$

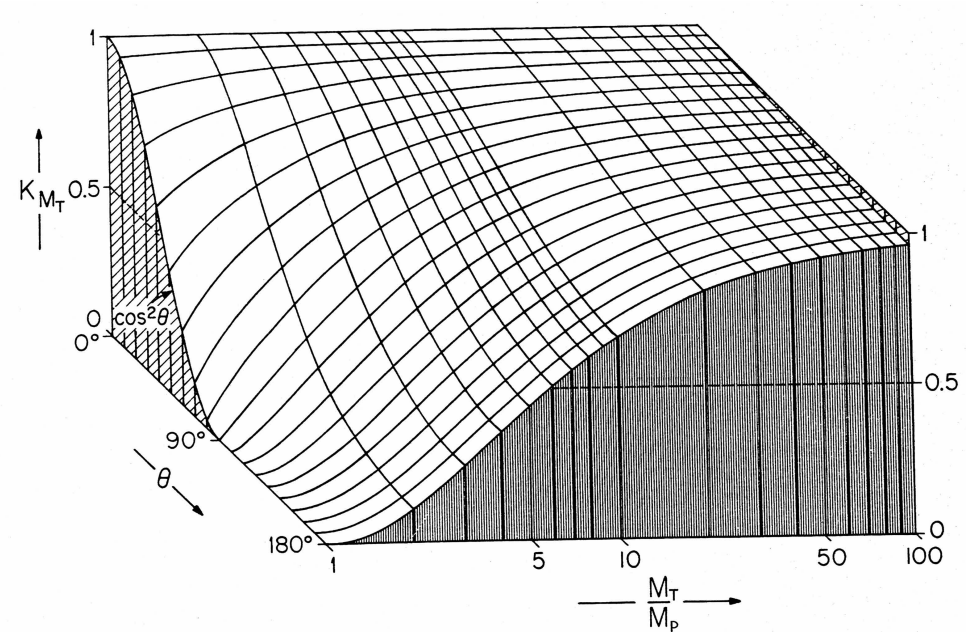

Abb. 3.8.: Abhähngigkeit des kinematischen Faktors vom Massenverhältnis $M_{P} / M_{T}$ und vom Streuwinkel $\theta$ [151.

Durch die Energiedifferenz $\Delta E$ lässt sich die Tiefe bestimmen, aus der ein Ion aus der Probe zurückgestreut wurde. Eine entscheidende Größe für die mathematische Beschreibung der Rutherford-Rückstreuung ist die differentielle Streuquerschnitt $\frac{\mathrm{d} \sigma}{\mathrm{d} \Omega}$. Der differentielle Streuquerschnitt gibt die Wahrscheinlichkeit an, dass ein Ion in den Raumwinkel $d \Omega$ gestreut wird und ist für das ungeschirmte Coulomb-Potential im Laborsystem durch

$$
\frac{\mathrm{d} \sigma}{\mathrm{d} \Omega}=\left(\frac{Z_{P} Z_{T} e^{2}}{16 \pi \epsilon_{0} E_{\mathrm{vor}}}\right)^{2} \cdot \frac{4}{\sin ^{4} \theta} \cdot \frac{\left(\sqrt{1-\left[\left(\frac{M_{P}}{M_{T}}\right) \sin \theta\right]^{2}}+\cos \theta\right)^{2}}{\sqrt{1-\left[\left(\frac{M_{P}}{M_{T}}\right) \sin \theta\right]^{2}}}
$$

gegeben. Hierbei sind $Z_{I}$ und $Z_{T}$ die Kernladungszahlen von Ion und Targetatom, $E_{\text {vor }}$ ist die Energie des Ions vor der Streuung und $\theta$ ist der Streuwinkel [53, 151].

Für $M_{P} \ll M T$ reduziert sich die Gleichung $3.8 \mathrm{zu}$

$$
\frac{\mathrm{d} \sigma}{\mathrm{d} \Omega}=\left(\frac{Z_{P} Z_{T} e^{2}}{16 \pi \epsilon_{0} E_{\mathrm{vor}}}\right)^{2} \cdot \frac{1}{\sin ^{4}\left(\frac{\theta}{2}\right)}
$$


Abbildung (3.9)zeigt ein RBS-Spektrum von $200 \mathrm{~nm}$ dicken Ge-Dünnschicht auf Si-Substrat. Die Germanium-Schicht wurde unter Verwendung eines $900 \mathrm{keV} \mathrm{He}{ }^{2+}$-Strahls analysiert.

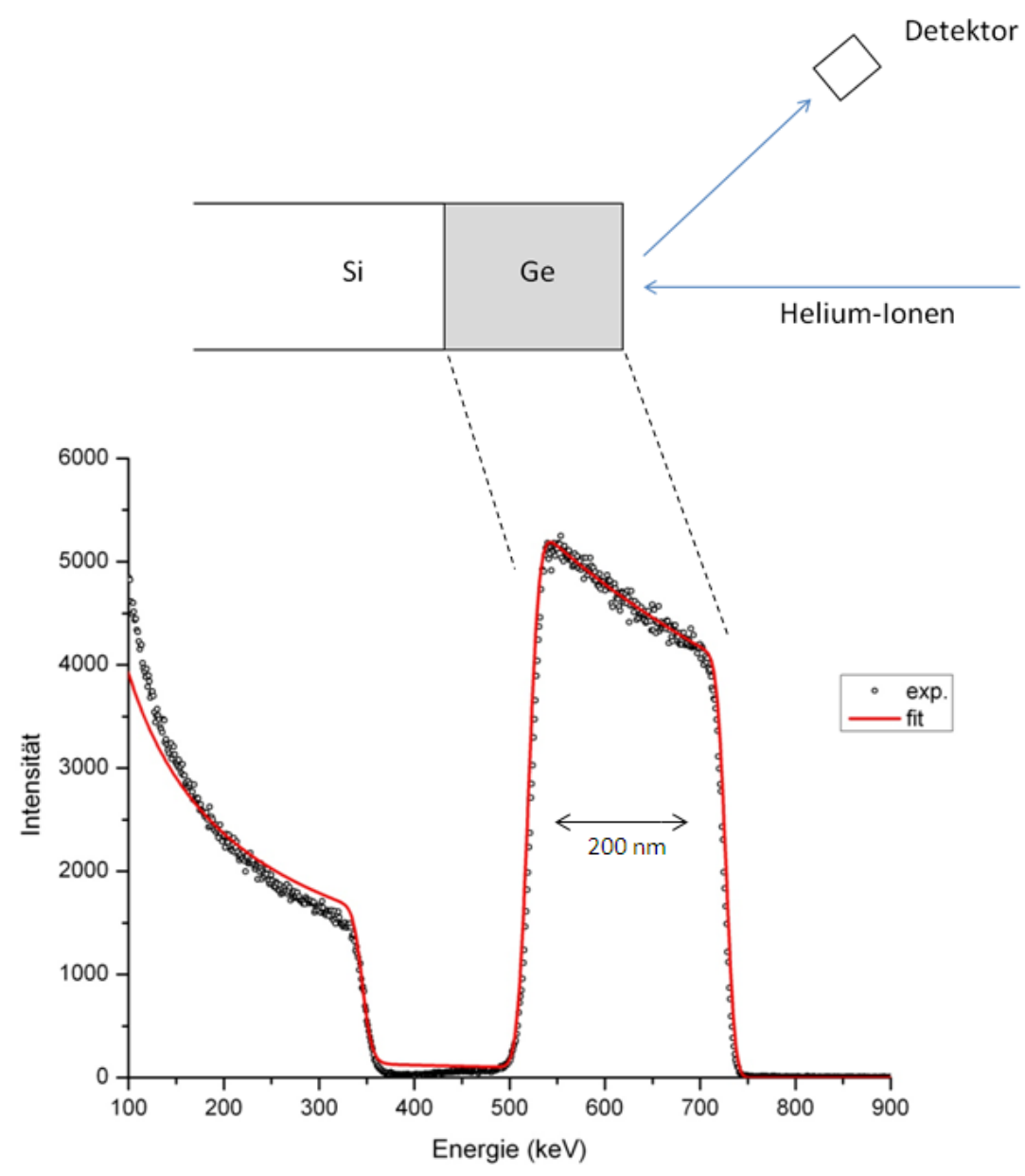

Abb. 3.9.: RBS-Spektrum von $200 \mathrm{~nm}$ dicken Germanium auf Si-Substrat. Die Kreise stellen die experimentellen Daten dar. Die rote Linie ist das simulierte Spektrum. 


\section{Ionenstrahlinduzierte Musterbildung auf einfachen Oberflächen}

\subsection{Einführung}

Obwohl zahlreiche experimentelle Untersuchungen mit $\mathrm{Ar}^{+}$-Ionenbestrahlung durchgeführt wurden [8, 13, 46, 83, 94, 124, 160], wurden nur sehr wenige Studien für leichte Ionen wie Neon durchgeführt. Nach Vishnyakov-Carter kann die Verwendung von leichten Ionen und niedrigen Energien Oberflächenwellen und anschließende große Amplitudenfacettierung auf Silizium bei Raumtemperatur hemmen [149]. Ziberi et al. berichteten, dass keine Musterbildung mit $\mathrm{Ne}^{+}$-Ionen bestrahlten Si-Oberflächen für Ionenenergie unter $2000 \mathrm{eV}$ beobachtet wurde, aber es werden keine Daten in der Arbeit gezeigt[159]. Zhu et al. beobachtete jedoch periodische Wellen auf diamantähnlichen Kohlenstoffschichten (DCL), die mit $1500 \mathrm{eV} \mathrm{Ne}^{+}$-Ionen und einem Einfallswinkel von $60^{\circ}$ bei $700^{\circ} \mathrm{C}$ bestrahlt wurden [158]. In diesem Kapital soll untersucht werden, woran es liegt, dass die Musterbildung nach Bestrahlung mit leichten Ionen wie $\mathrm{Ne}^{+}$möglicherweise unterdrückt ist. Um dieses Ziel zu erreichen, wird die ioneninduzierte Musterbildung auf amorphen Kohlenstoffund kristallinen Siliziumoberflächen bei Bestrahlung mit niederenergetischen $\mathrm{Ne}^{+}$- und $\mathrm{Ar}^{+}$-Ionen von $250 \mathrm{eV}$ bis zu $60 \mathrm{keV}$ untersucht.

\subsection{Ionenstrahlinduzierte Musterbildung auf a-C Schichten}

\subsubsection{Bestrahlung mit $\mathrm{Ne}^{+}-$Ionen}

Die Abhängigkeit der Oberflächenmorphologie vom globalen Einfallswinkel $\theta$ nach Ionenbeschuss mit unterschiedlicher Energie ist in Abbildung 4.1) dargestellt. Für globale Einfallswinkel im Bereich von $\theta \leq 45^{\circ}$ bilden sich keine Muster und die Rauigkeit bleibt 
sehr gering mit $\omega_{\mathrm{rms}}=0.2 \mathrm{~nm}-0.5 \mathrm{~nm}$.

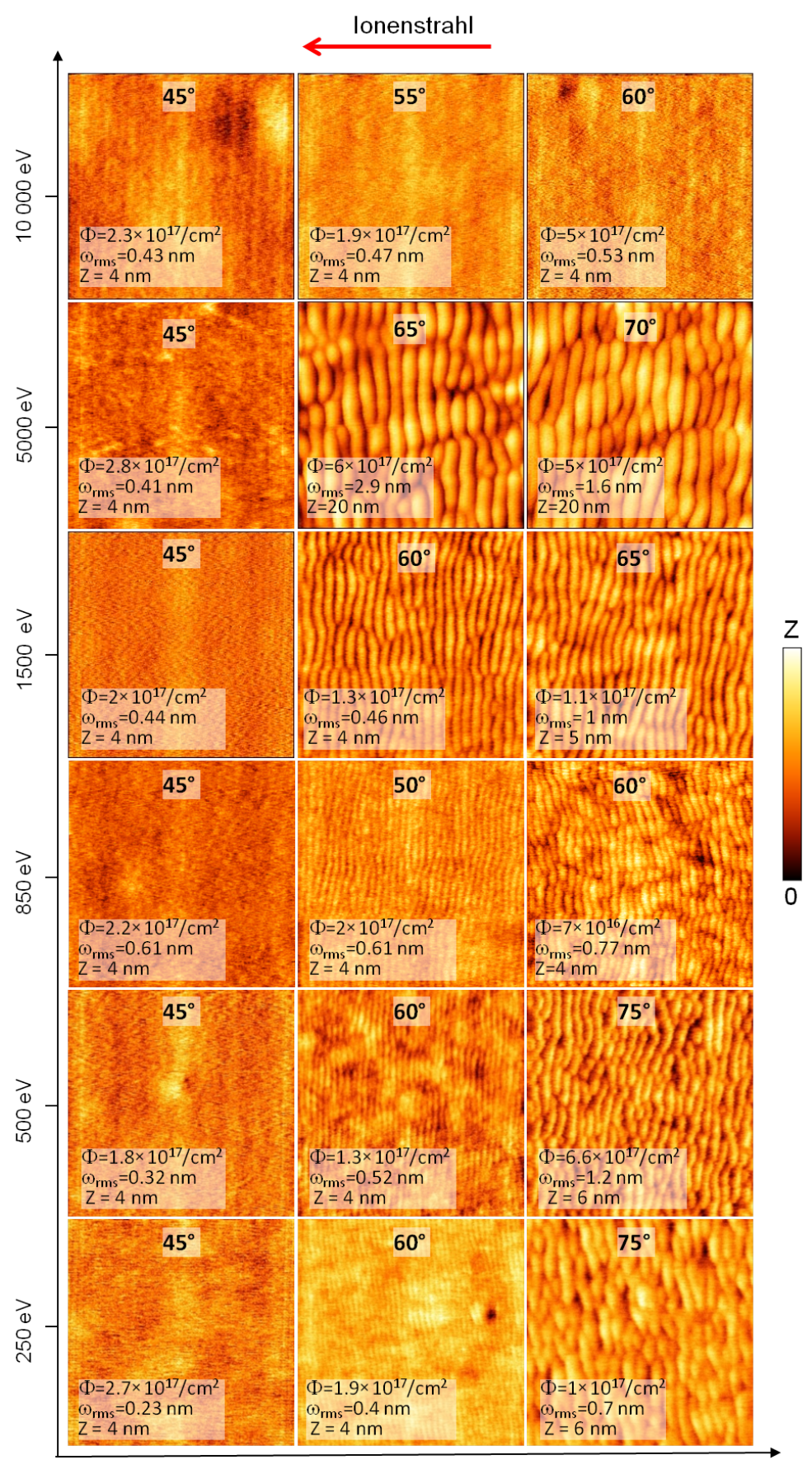

Einfallswinkel

Abb. 4.1.: AFM-Aufnahmen von ta-C Schichten nach der Bestrahlung von $\mathrm{Ne}^{+}$-Ionen. Jedes Bild zeigt einen $500 \times 500 \mathrm{~nm}^{2}$ großen Ausschnitt, außer bei 5 und 10 $\mathrm{keV} 1 \times 1 \mu \mathrm{m}^{2}$. 
Für $50^{\circ} \leq \theta \leq 60^{\circ}$ und Ionenenergien zwischen $250 \mathrm{eV}$ und $5 \mathrm{keV}$ wurden Rippelmuster mit kleiner Amplitude und Wellenvektor $\vec{k}$ parallel zur Ionenstrahlrichtung gebildet. Ausgeprägte Musterbildung findet in einem engen Winkelbereich zwischen $65^{\circ}$ und $75^{\circ}$ statt. Für die wohlgeordneten Wellen, die bei einem schrägen Ionenstrahl erzeugt werden, erhalten wir eine mittlere Wellenlängen zwischen $16 \mathrm{~nm}$ und $73 \mathrm{~nm}$. Eine Zusammenstellung dieser experimentellen Daten führt zur Abbildung (4.2). Die Wellenlängen sind als Funktion der Ionenenergie und des Ioneneinfallswinkels aufgetragen. In den Fällen, in denen keine Rippelmuster beobachtet wurden, ist der Datenpunkt in der unteren Ebene aufgetragen. Für Bestrahlung mit $10 \mathrm{keV}$ und Fluenz bis zu $5 \times 10^{17} / \mathrm{cm}^{2}$ bleibt die Oberfläche glatt mit einer Rauigkeit bis $\mathrm{zu} \omega_{\text {rms }}=0.5 \mathrm{~nm}$.

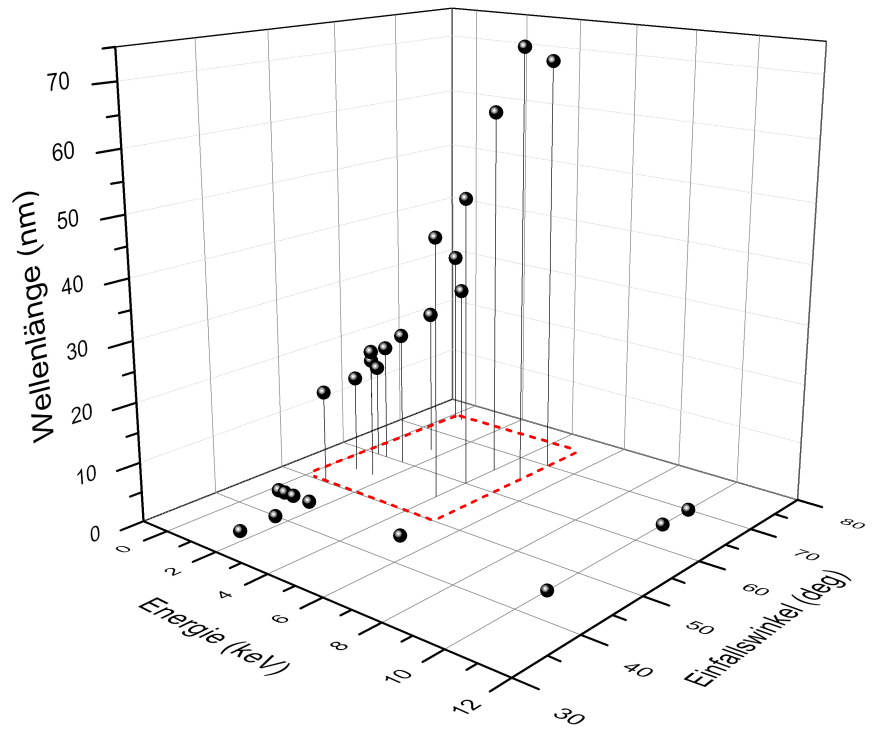

$\boldsymbol{A} \boldsymbol{b} \boldsymbol{b}$. 4.2.: Diagramm der gemessenen Wellenlängen für die $\mathrm{Ne}^{+}$-Bestrahlung mit Energien zwischen $250 \mathrm{eV}$ und $10 \mathrm{keV}$ als Funktion der Ionenenergie und des Ioneneinfallswinkels. Der gestrichelte rechteckige Bereich markiert den Bereich, in dem Rippelmuster beobachtet wurden.

\subsubsection{Simulationsergebnisse}

Die Monte-Carlo-Simulation der Krümmungskoeffizienten $C_{11}^{\text {totat }}$ ist in den Abbildungen 4.3, 4.4 und 4.5 dargestellt. Die Simulationen wurden hauptsächlich im dynamischen Modus durchgeführt, um den Ionenimplantationseffekt zu berücksichtigen. Um die Reinheit der bestrahlten Proben zu verifizieren und den restlichen Ne-Gehalt zu messen, 


\section{Ionenstrahlinduzierte Musterbildung auf einfachen Oberflächen}

wurden die Proben mit Rutherford-Rückstreu-Spektroskopie (RBS) unter Verwendung eines $900 \mathrm{keV} \mathrm{He}{ }^{2+}$-Strahls analysiert. Tabelle (4.1) zeigt die Ionenimplantation und den restlichen Ne-Gehalt einiger bestrahlter Proben, die mit SDTrimSP-Programm berechnet wurden, verglichen mit der RBS-Analyse des Restgehaltes von Ne in bestrahlten ta-CSchichten. Für Ionenenergien zwischen $250 \mathrm{eV}$ und $10 \mathrm{keV}$ ist der Koeffizient

$$
C_{11}^{\text {total }}=C_{11}^{\text {eros. }}+C_{11}^{\text {redist. }}+C_{11}^{\text {implant. }}+T_{11}+D_{11}
$$

für Einfallswinkel zwischen $40^{\circ}$ und ca. $80^{\circ}$ negativ, was eine Instabilität der Oberfläche in $x$-Richtung verursacht und somit zu parallelen Rippelmustern führt. Der Winkelbereich ist in Abbildungen (4.3), 4.4) und (4.5) als grau schattierte Fläche markiert. Wenn der dickenabhängige Krümmungskoeffizient $D_{11}$ berücksichtigt wird, wird die Summe des erosiven, des redistributiven und des Implantationsterms für Einfallswinkel zwischen etwa $55^{\circ}$ und $85^{\circ}$ negativ und man würde nur parallele Rippelmuster ab etwa $55^{\circ}$ erwarten. Die berechneten Krümmungskoeffizienten zeigen auch, dass bei vernachlässigter Ionenimplantation nur ein positiver Gesamtkrümmungskoeffizient für die mit $1.5 \mathrm{keV}$ und $5 \mathrm{keV}$ Ionen bestrahlten Proben und ein sehr kleiner negativer Gesamtkrümmungskoeffizient für 850 eV und $10 \mathrm{keV}$ Ionenbestrahlung zu erwarten sind. Das bedeutet, dass keine Rippelmuster

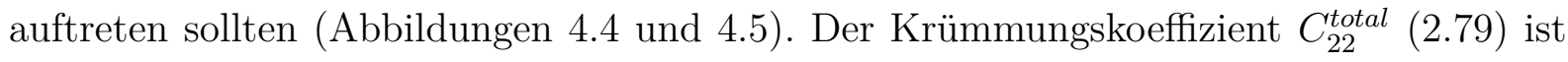
für die Energien $250 \mathrm{eV}$ bis $10 \mathrm{keV}$ in den Abbildungen (4.6), 4.7) und (4.8) gezeigt. Ionenimplantation führt zu einer signifikanten Stabilisierung der Oberfläche gegen senkrechte Rippelnmusterbildung für alle Einfallswinkel und für alle Energien zwischen 250 eV und $10 \mathrm{keV}$.

\begin{tabular}{ccccccc}
\hline $\begin{array}{c}\mathrm{E}_{\text {ion }} \\
{[\mathrm{eV}]}\end{array}$ & $\begin{array}{c}\theta \\
{[\mathrm{Grad}]}\end{array}$ & $\begin{array}{c}\text { Fluenz } \\
{\left[\text { Ionen } / \mathrm{cm}^{2}\right]}\end{array}$ & $\begin{array}{c}\text { Ne-Implant. } \\
\text { SDTrimSP }\end{array}$ & $\begin{array}{c}\text { Ne-gesputt. } \\
\text { SDTrimSP }\end{array}$ & $\begin{array}{c}\text { Ne-Gehalt } \\
\text { SDTrimSP }\end{array}$ & $\begin{array}{c}\text { Ne-Gehalt } \\
\text { RBS }\end{array}$ \\
\hline 250 & $60^{\circ}$ & $1.9 \times 10^{17}$ & $66.8 \%$ & $65.5 \%$ & $1.3 \%$ & $1.3 \%$ \\
500 & $60^{\circ}$ & $1.3 \times 10^{17}$ & $38.1 \%$ & $36.8 \%$ & $1.3 \%$ & $1.3 \%$ \\
850 & $55^{\circ}$ & $1.8 \times 10^{17}$ & $32.9 \%$ & $31.5 \%$ & $1.4 \%$ & $1.4 \%$ \\
1500 & $65^{\circ}$ & $1 \times 10^{17}$ & $34.8 \%$ & $32.4 \%$ & $2.4 \%$ & $2.4 \%$ \\
\hline
\end{tabular}

Tab. 4.1.: Konzentration von implantierten Ne-Ionen und des restlichen Ne-Gehalts, berechnet mit SDTrimSP. Die fünfte Spalte zeigt den Anteil der durch die Ionenbestrahlung wieder gesputterten Ne-Atomen. Die letzte Spalte zeigt den gemessenen restlichen Ne-Gehalt unter Verwendung der Rutherford-RückstreuSpektroskopie. Der Ne-Gehalt ist bezogen auf die Fluenz in der dritten Spalte. 
$250 \mathrm{eV}$ Ne auf ta-C

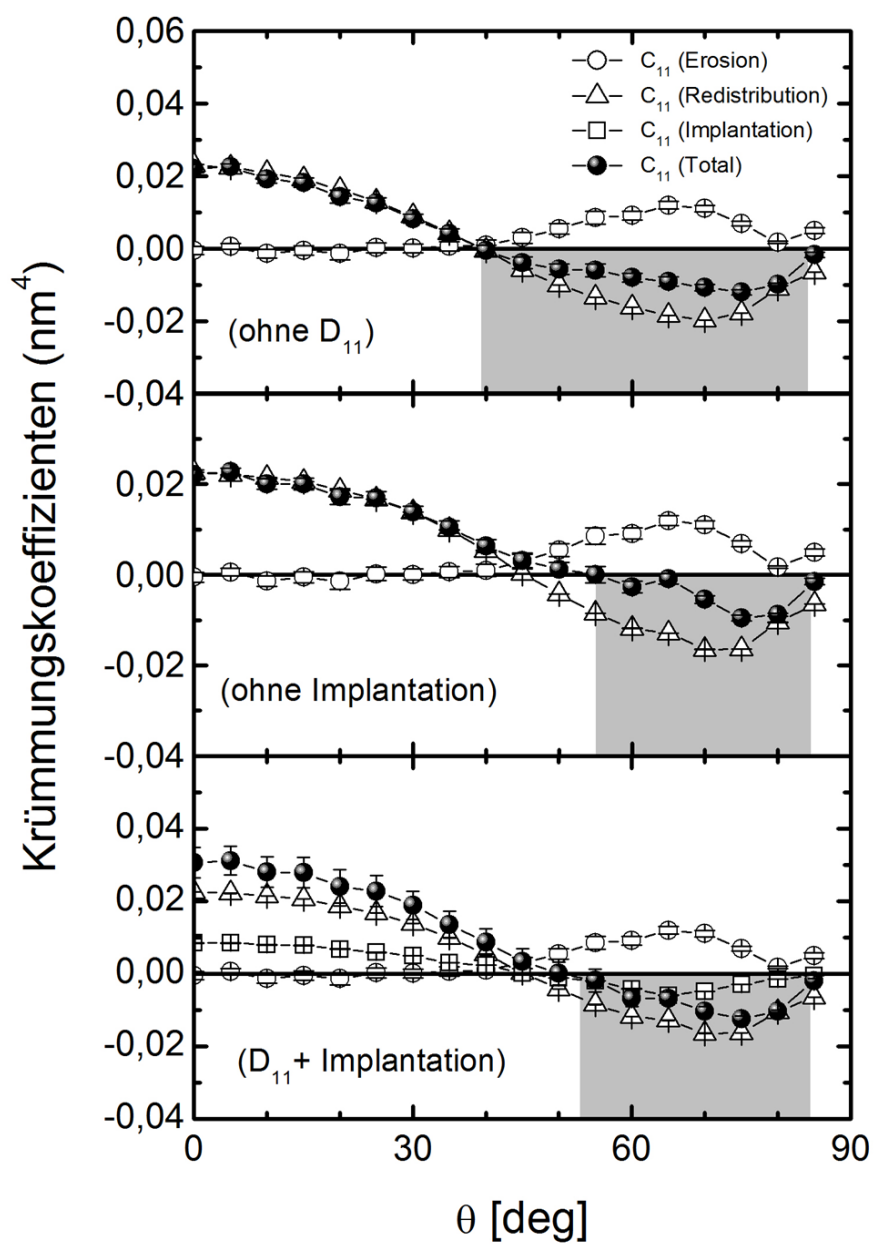

$500 \mathrm{eV} \mathrm{Ne}$ auf ta-C

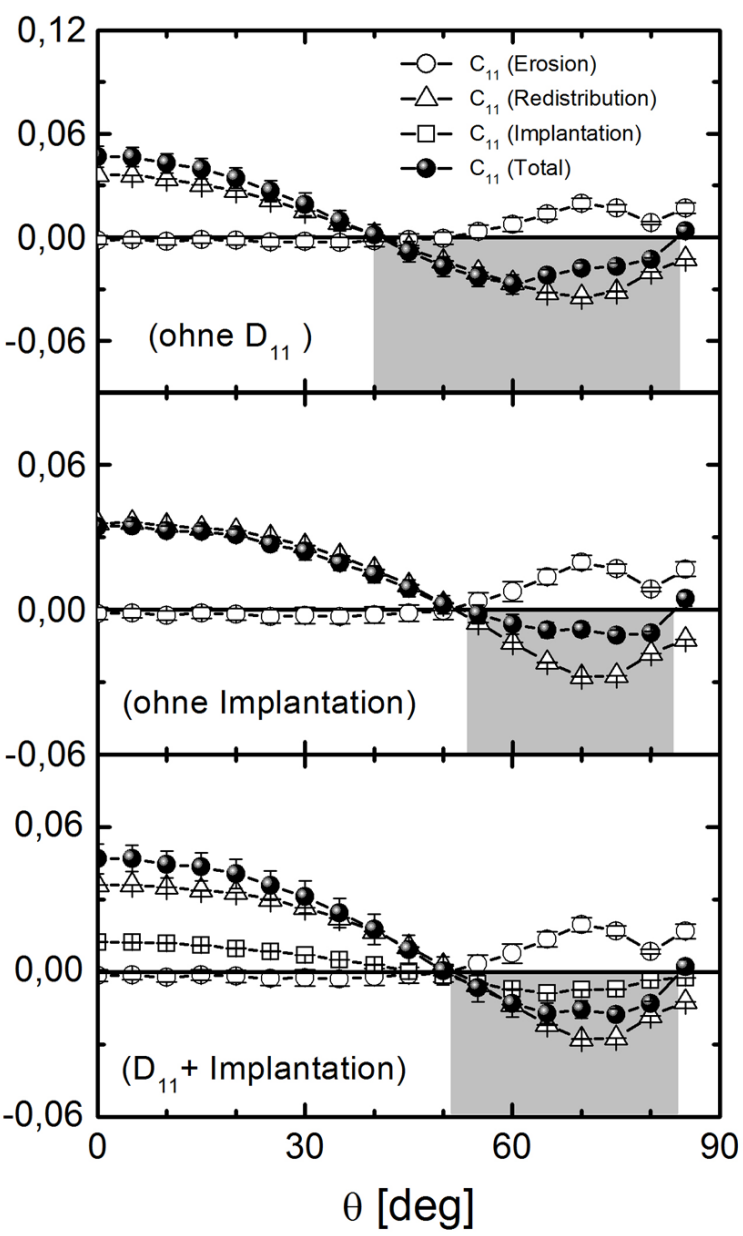

Abb. 4.3.: Berechnete Krümmungskoeffizienten $C_{11}^{\text {total }}$ für $250 \mathrm{eV}$ und $500 \mathrm{eV} \mathrm{Ne} \mathrm{Ne}^{+}$ Ionenbestrahlung von ta-C. Der Erosionskoeffizient $C_{11}^{\text {eros. }}$ beinhaltet die Krümmungsabhängigkeit der Erosionskraterfunktion $T_{11}$. Im oberen Diagramm beinhaltet $C_{11}^{\text {total }}$ den Effekt der Ionenimplantation $C_{11}^{\text {implant. }}$, aber die Dickenanhängigkeit der bestrahlten Schicht $D_{11}$ ist vernachlässigt. Im mittleren Diagramm beinhaltet $C_{11}^{\text {total }}$ die Dickenabhängigkeit $D_{11}$, aber der Effekt der Ionenimplantation $C_{11}^{\text {implant. }}$ ist nicht berücksichtigt. Im unteren Diagramm sind die beiden Koeffizienten $D_{11}$ und $C_{11}^{\text {implant. }}$ berücksichtigt. Der grau schattierte Bereich zeigt die Winkelbereiche an, in denen $C_{11}^{\text {total }}<0$ ist. 
$850 \mathrm{eV} \mathrm{Ne}$ auf ta-C

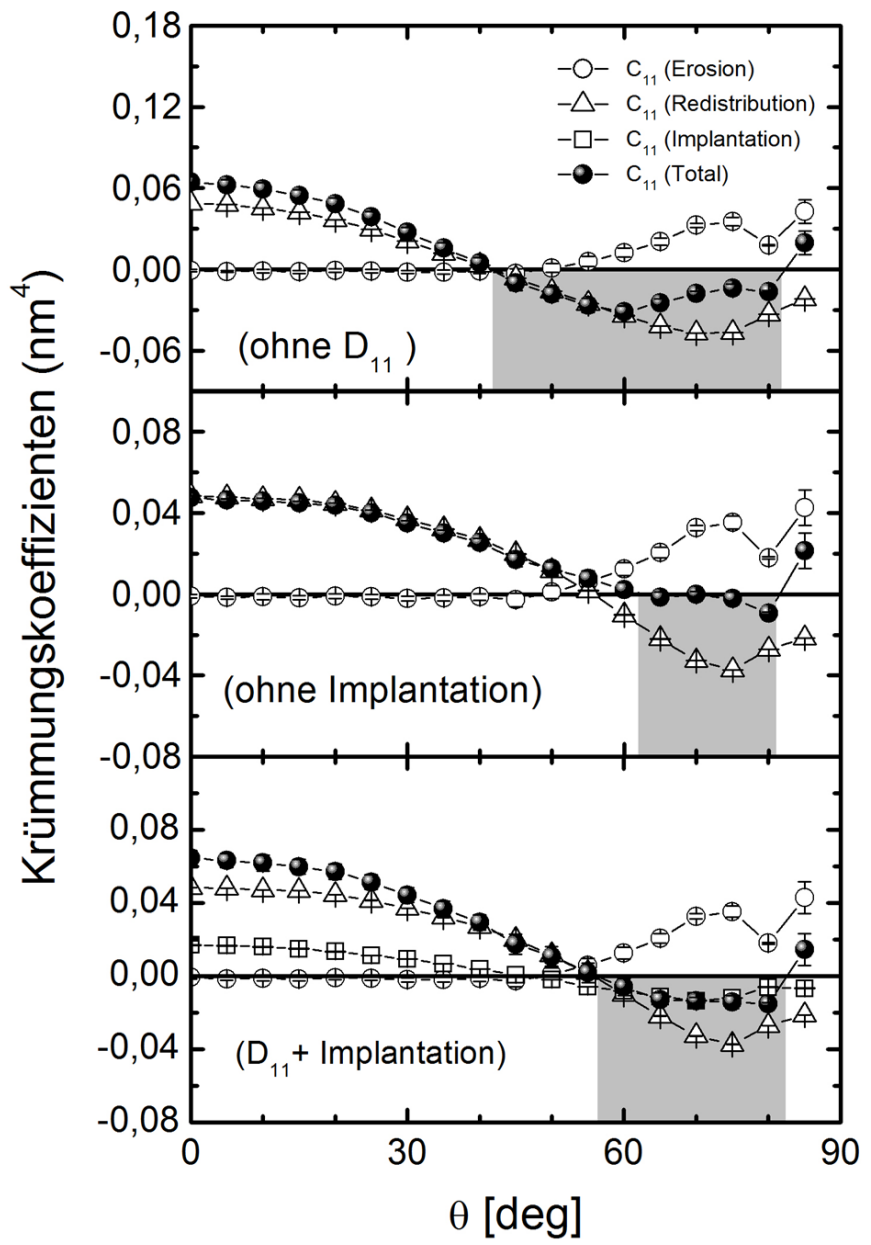

$1500 \mathrm{eV} \mathrm{Ne}$ auf ta-C

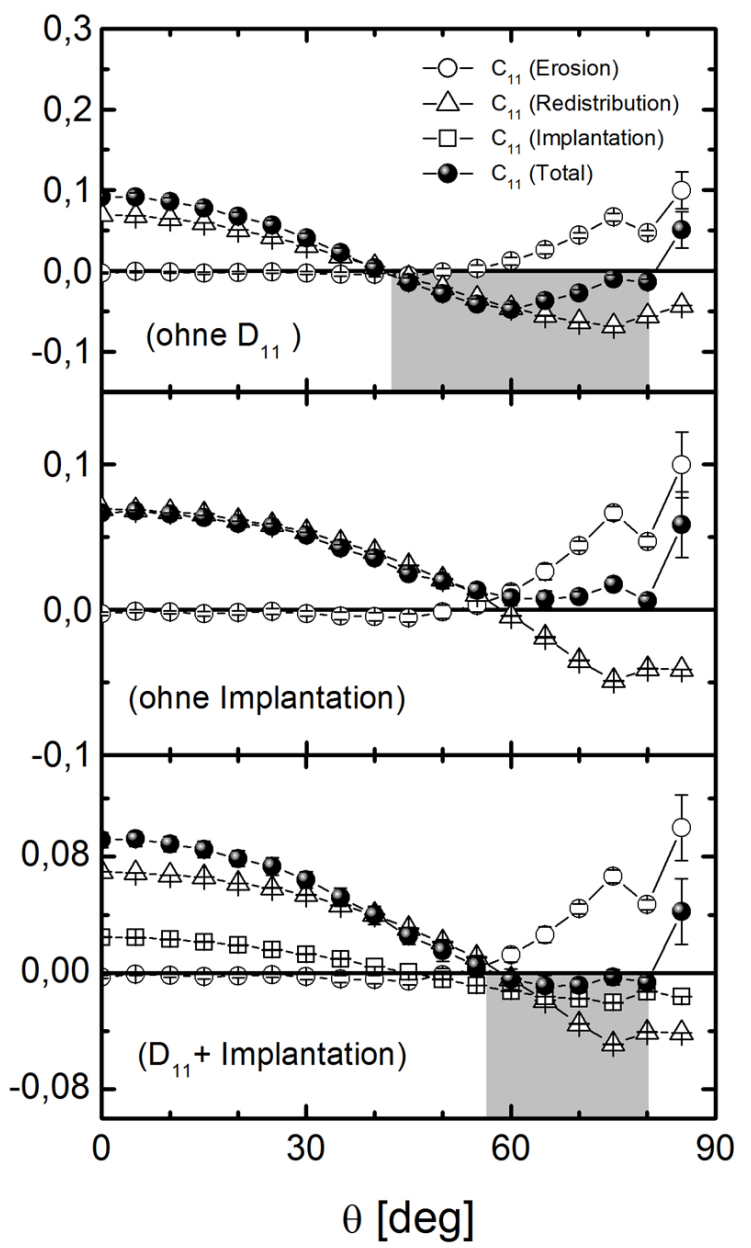

Abb. 4.4.: Berechnete Krümmungskoeffizienten $C_{11}^{\text {total }}$ für $850 \mathrm{eV}$ und $1.5 \mathrm{keV} \mathrm{Ne} \mathrm{Ne}^{+}$ Ionenbestrahlung von ta-C. Der Erosionskoeffizient $C_{11}^{\text {eros. }}$ beinhaltet die Krümmungsabhängigkeit der Erosionskraterfunktion $T_{11}$. Im oberen Diagramm beinhaltet $C_{11}^{\text {total }}$ den Effekt der Ionenimplantation $C_{11}^{\text {implant. }}$, aber die Dickenanhängigkeit der bestrahlten Schicht $D_{11}$ ist vernachlässigt. Im mittleren Diagramm beinhaltet $C_{11}^{\text {total }}$ die Dickenabhängigkeit $D_{11}$, aber der Effekt der Ionenimplantation $C_{11}^{\text {implant. }}$ ist nicht berücksichtigt. Im unteren Diagramm sind die beiden Koeffizienten $D_{11}$ und $C_{11}^{\text {implant. }}$ berücksichtigt. Der grau schattierte Bereich zeigt die Winkelbereiche an, in denen $C_{11}^{\text {total }}<0$ ist. 
$5 \mathrm{keV} \mathrm{Ne}$ auf ta-C

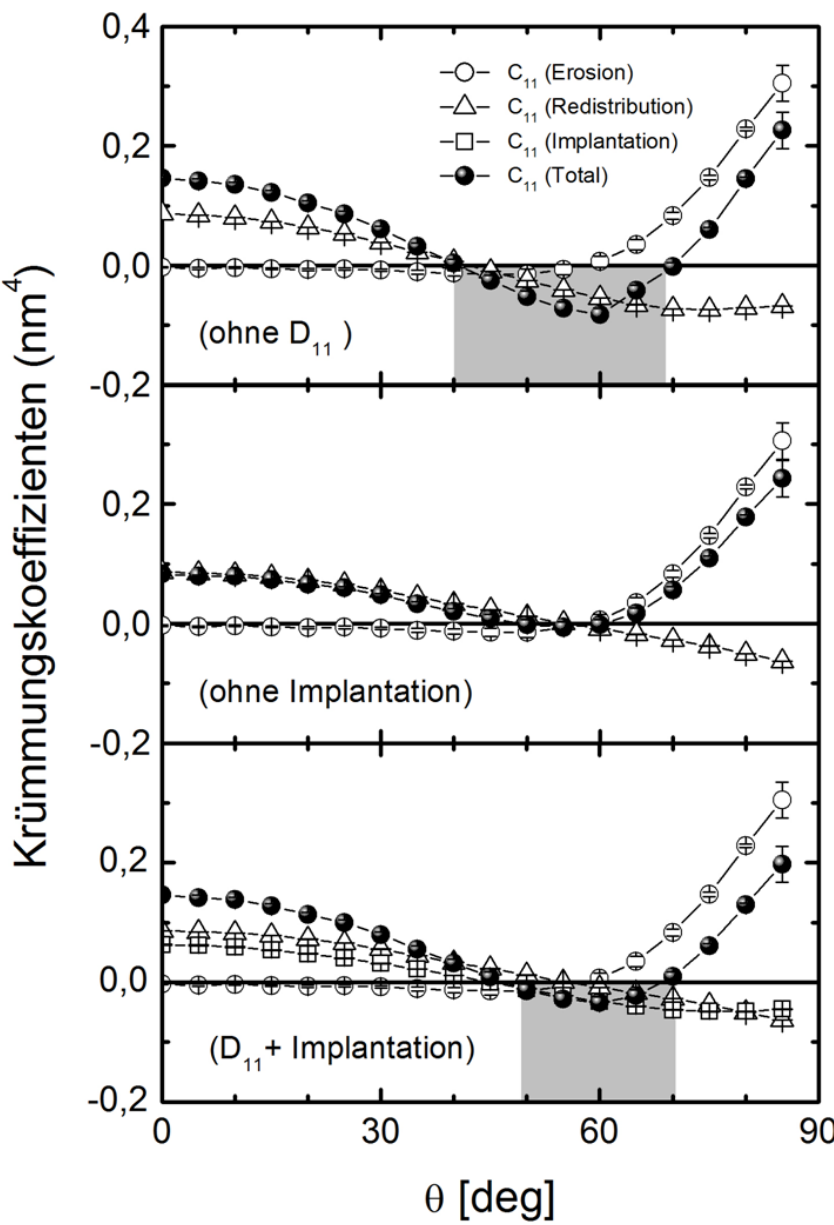

$10 \mathrm{keV} \mathrm{Ne}$ auf ta-C

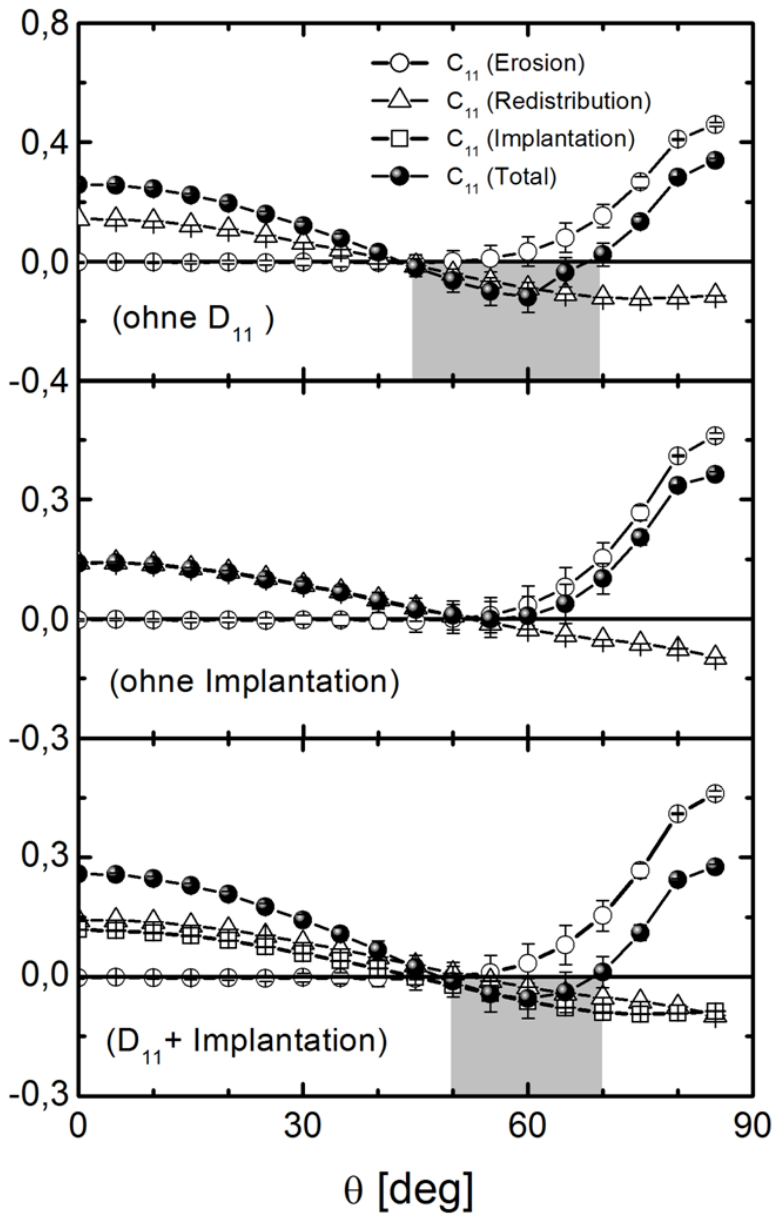

Abb. 4.5.: Berechnete Krümmungskoeffizienten $C_{11}^{\text {total }}$ für $5 \mathrm{keV}$ und $10 \mathrm{keV} \mathrm{Ne}^{+}$ Ionenbestrahlung von ta-C. Der Erosionskoeffizient $C_{11}^{\text {eros. }}$ beinhaltet die Krümmungsabhängigkeit der Erosionskraterfunktion $T_{11}$. Im oberen Diagramm beinhaltet $C_{11}^{\text {total }}$ den Effekt der Ionenimplantation $C_{11}^{\text {implant. }}$, aber die Dickenanhängigkeit der bestrahlten Schicht $D_{11}$ ist vernachlässigt. Im mittleren Diagramm beinhaltet $C_{11}^{\text {total }}$ die Dickenabhängigkeit $D_{11}$, aber der Effekt der Ionenimplantation $C_{11}^{\text {implant. }}$ ist nicht berücksichtigt. Im unteren Diagramm sind die beiden Koeffizienten $D_{11}$ und $C_{11}^{\text {implant. }}$ berücksichtigt. Der grau schattierte Bereich zeigt die Winkelbereiche an, in denen $C_{11}^{\text {total }}<0$ ist. 

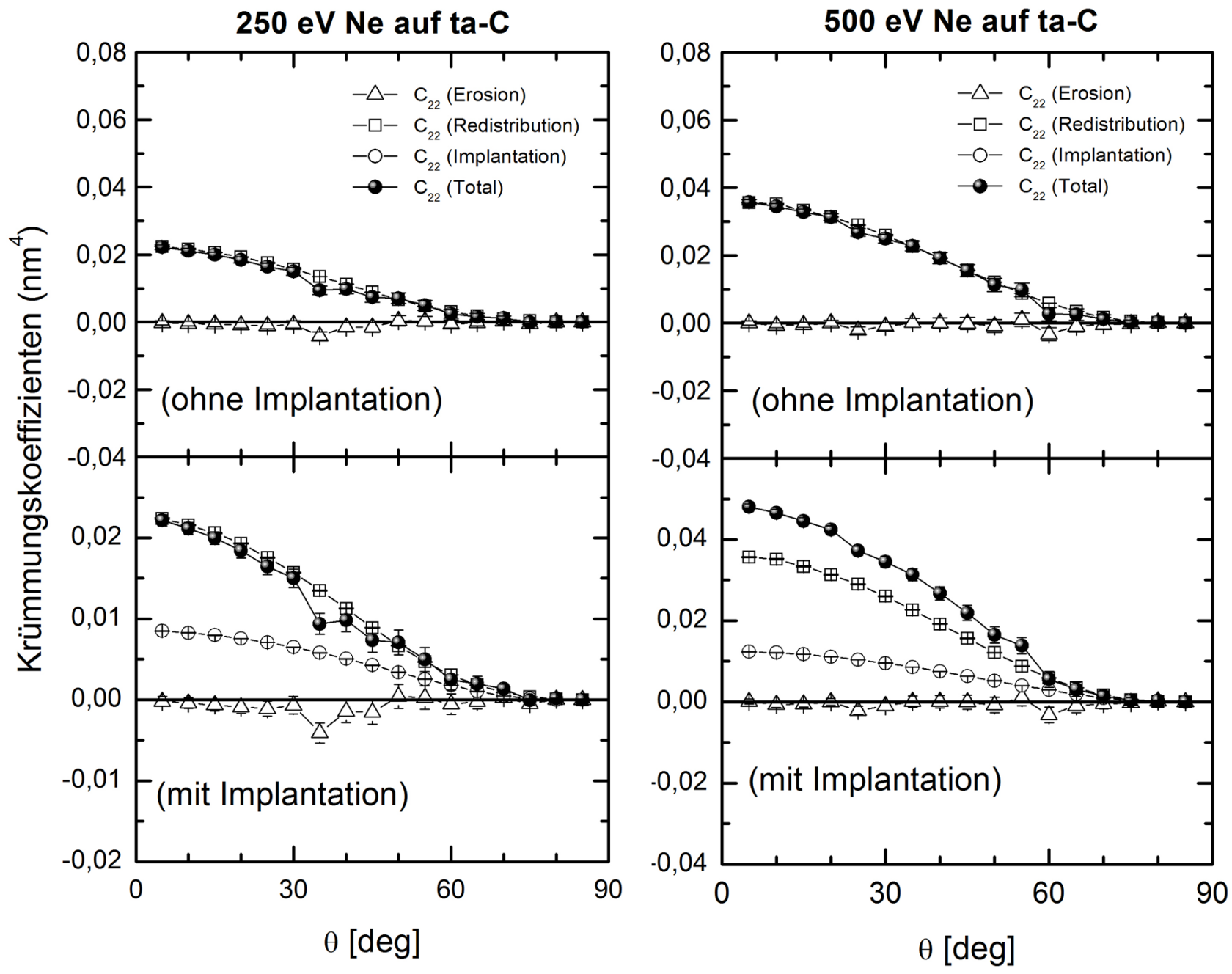

Abb. 4.6.: Berechnete Krümmungskoeffizienten $C_{22}^{\text {total }}$ für $250 \mathrm{eV}$ und $500 \mathrm{eV} \mathrm{Ne} \mathrm{Ne}^{+}$ Ionenbestrahlung von ta-C. Der Erosionskoeffizient $C_{22}^{\text {eros. }}$ beinhaltet die Krümmungsabhängigkeit der Erosionskraterfunktion $T_{22}$. Im oberen Diagramm beinhaltet $C_{22}^{\text {total }}$ den Effekt der Ionenimplantation $C_{22}^{\text {implant. }}$ Im unteren Diagramm wird der Effekt der Ionenimplantation nicht berücksichtigt. 

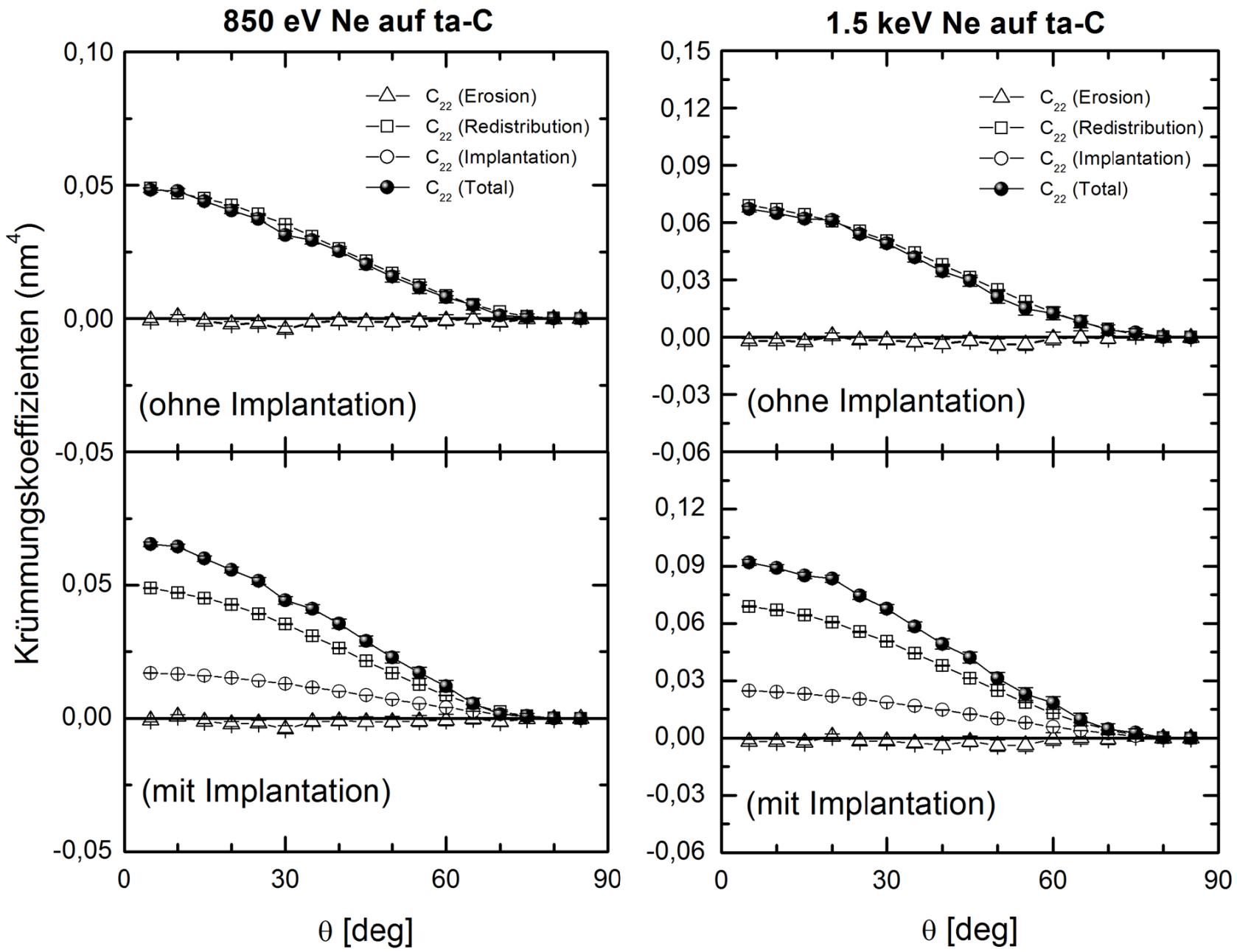

Abb. 4.7.: Berechnete Krümmungskoeffizienten $C_{22}^{\text {total }}$ für $850 \mathrm{eV}$ und $1.5 \mathrm{keV} \mathrm{Ne}^{+}$ Ionenbestrahlung von ta-C. Der Erosionskoeffizient $C_{22}^{\text {eros. }}$ beinhaltet die Krümmungsabhängigkeit der Erosionskraterfunktion $T_{22}$. Im oberen Diagramm beinhaltet $C_{22}^{\text {total }}$ den Effekt der Ionenimplantation $C_{22}^{\text {implant. }}$. Im unteren Diagramm wird der Effekt der Ionenimplantation nicht berücksichtigt. 

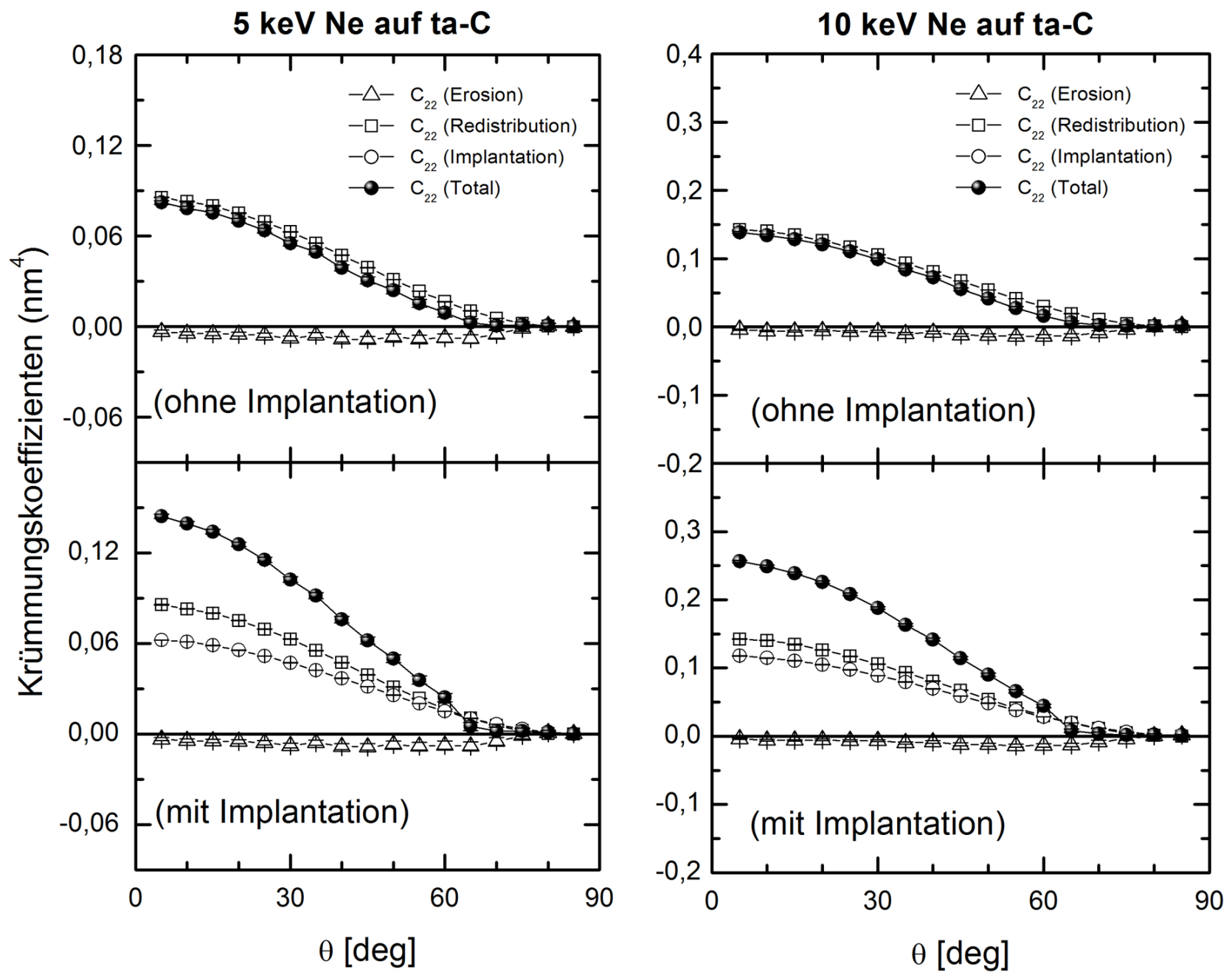

Abb. 4.8.: Berechnete Krümmungskoeffizienten $C_{22}^{\text {total }}$ für $5 \mathrm{keV}$ und $10 \mathrm{keV} \mathrm{Ne}^{+}$ Ionenbestrahlung von ta-C. Der Erosionskoeffizient $C_{22}^{\text {eros. }}$ beinhaltet die Krümmungsabhängigkeit der Erosionskraterfunktion $T_{22}$. Im oberen Diagramm beinhaltet $C_{22}^{\text {total }}$ den Effekt der Ionenimplantation $C_{22}^{\text {implant. }}$ Im unteren Diagramm wird der Effekt der Ionenimplantation nicht berücksichtigt. 


\subsubsection{Bestrahlung mit $\mathrm{Ar}^{+}$-Ionen}

Im Folgenden wird die Musterbildung auf ta-C Schichten mit $\mathrm{Ar}^{+}$-Ionenbestrahlung im Energiebereich $200 \mathrm{eV}$ bis $10 \mathrm{keV}$ und Einfallswinkel von $60^{\circ}$ diskutiert. Es wurde Rippelmuster orientiert mit Wellenvektor parallel zur projizierten Ionenstrahlrichtung für Ionenenergie bis zu $10 \mathrm{keV}$ beobachtet. Die AFM-Bilder von Abbildung (4.10) zeigen Rippelmuster auf ta-C, erhalten für eine Ionenfluenz zwischen $3,5 \times 10^{17}$ Ionen $/ \mathrm{cm}^{2}$ und $3,5 \times 10^{17}$ Ionen $/ \mathrm{cm}^{2}$. Die Wellenlänge nimmt mit zunehmender Ionenenergie von etwa $14 \mathrm{~nm}$ bis $68 \mathrm{~nm}$ zu und die effektive Rauigkeit der Muster beträgt etwa $0.5-1 \mathrm{~nm}$. Die Wellenlänge der in den AFM-Bildern von Abbildung (4.10) gezeigten Wellenmustern ist in Abbildung (4.9) als Funktion der Ionenenergie aufgetragen. Die Wellenlänge und die entsprechenden Fehlerbalken werden mit Hilfe der spektralen Leistungsdichte-Verteilung der AFM-Bilder (PSD) bestimmt. Es findet sich eine gute lineare Beziehung zwischen Wellenwellenlänge und Ionenenergie. Eine solche lineare Beziehung zwischen der RippelWellenlänge und der Ionenenergie wurde bereits für andere Oberflächen, wie $\mathrm{Si}$ [33], $\mathrm{SiO}_{2}$ [144] und Graphit [58, berichtet. Abbildung (4.11] zeigt die Winkelabhängigkeit von ta-CSchichten nach einer $\mathrm{Ar}^{+}$-Bestrahlung für Ionenenergie von $500 \mathrm{eV}, 1 \mathrm{keV}$ und $10 \mathrm{keV}$. Es wurde in den Experimenten einen Übergang von einer flachen Oberfläche zu senkrechten Rippelmustern um $60^{\circ}$ für Ionenenergien von $500 \mathrm{eV}$ und $1 \mathrm{keV}$ gefunden. Parallellrippel auf ta-C-Schichten wurden nach $10 \mathrm{keV} \mathrm{Ar}^{+}$-Bestrahlung bei $65^{\circ}$ beobachtet. Für Einfallswinkel $\leq 55^{\circ}$ bleibt die Oberfläche glatt mit einer Rauigkeit von etwa $0,5 \mathrm{~nm}$.

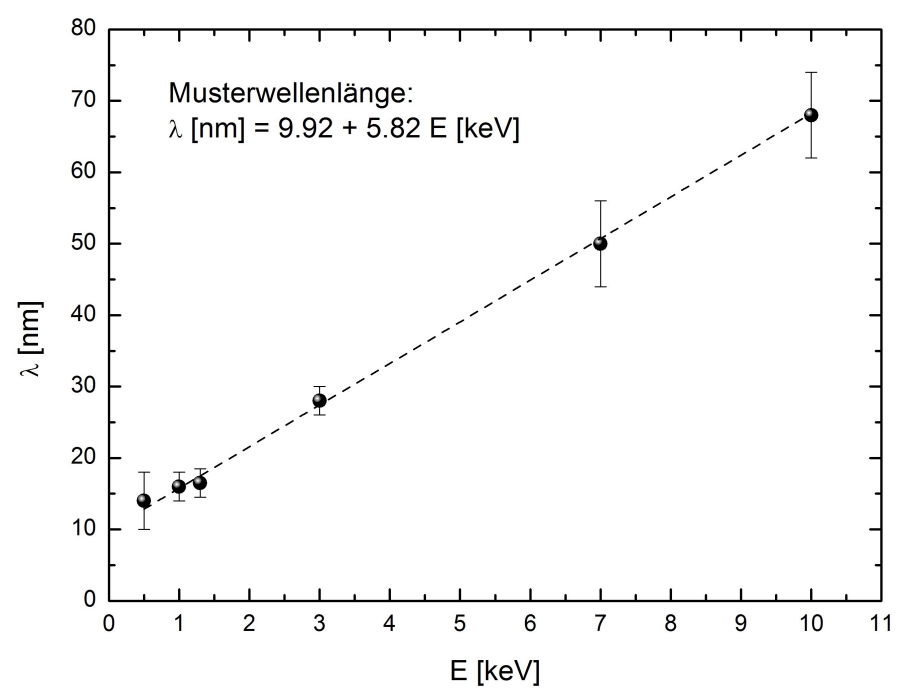

Abb. 4.9.: Wellenlänge der in Abbildung 4.10 gezeigten Rippelmuster als Funktion der Ionenenergie für einen festen Ioneneinfallswinkel von $60^{\circ}$. 


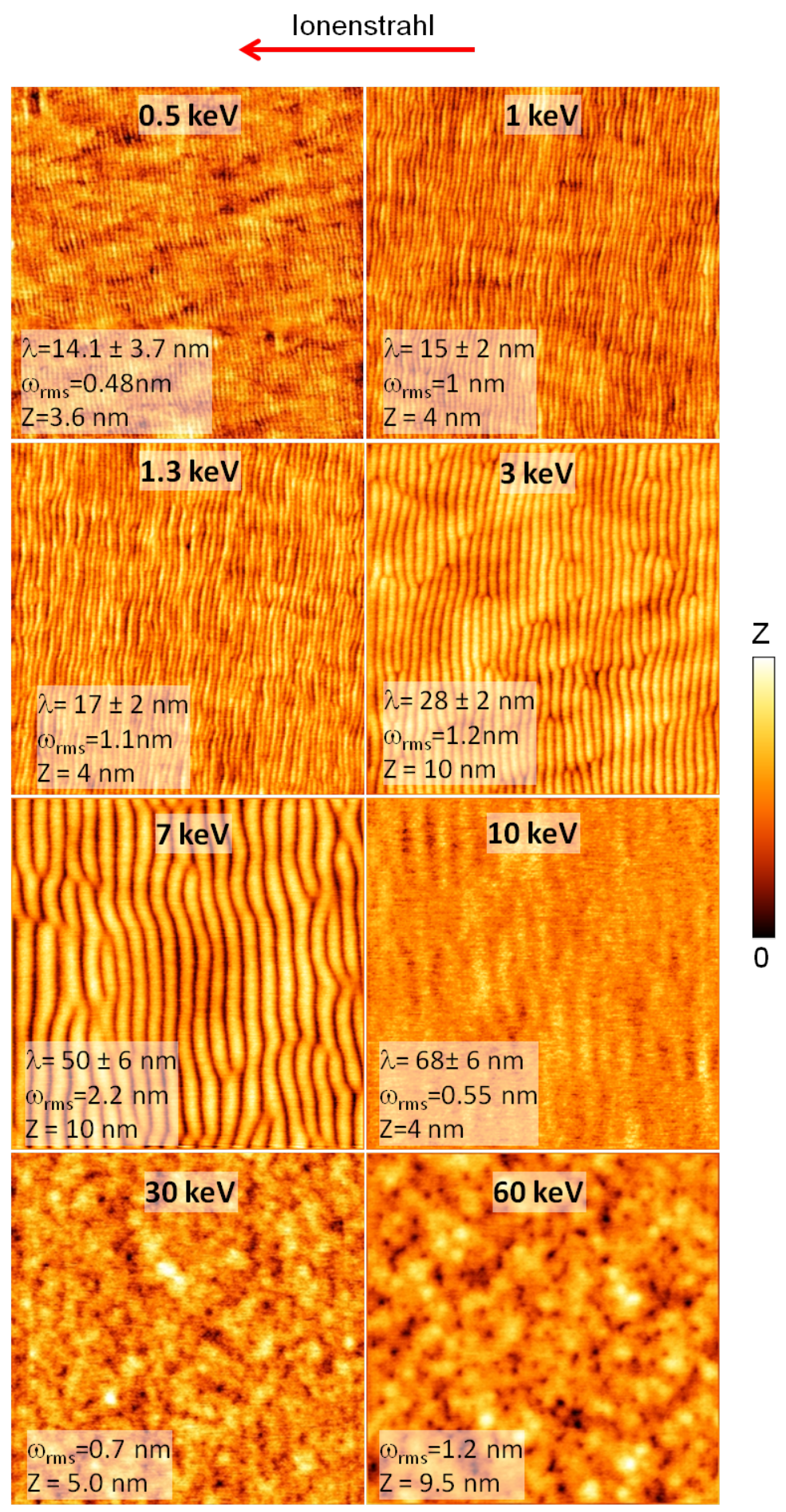

Abb. 4.10.: AFM-Bilder von Rippelmustern auf ta-C-Schichten, die mit $\mathrm{Ar}^{+}$-Ionen unter einem Einfallswinkel von $60^{\circ}$ und unterschiedlichen Ionenenergien zwischen $500 \mathrm{eV}$ und $60 \mathrm{keV}$ erodiert wurden. Die Rippel sind mit einem Wellenvektor parallel zur projizierten Ionenstrahlrichtung orientiert. Jedes Bild zeigt einen $1 \times 1 \mu \mathrm{m}^{2}$ großen Ausschnitt. 


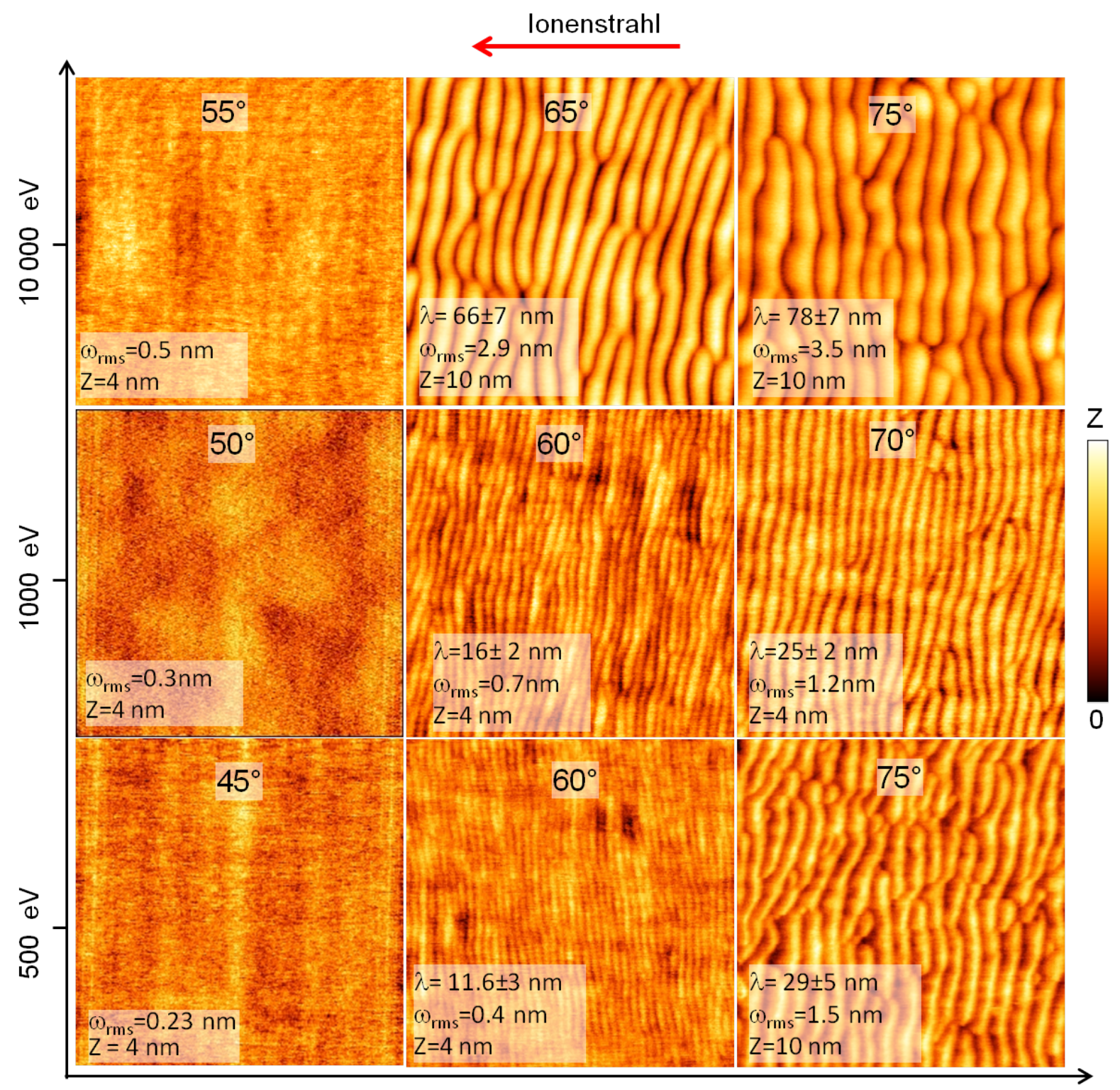

Einfallswinkel

Abb. 4.11.: AFM-Aufnahmen von den ta-C Schichten nach der Bestrahlung von $\mathrm{Ar}^{+}$ Ionen als Funktion des Einfallswinkels. Jedes Bild zeigt einen $500 \times 500 \mathrm{~nm}^{2}$ großen Ausschnitt außer bei $10 \mathrm{keV} 1 \times 1 \mu \mathrm{m}^{2}$.

\subsubsection{Simulationsergebnisse}

Die drei Krümmungskoeffizienten $C_{11}^{\text {eros. }}, C_{11}^{\text {redistr. }}$ und $C_{11}^{\text {total }}$ sind in Abbildung 4.12) und 4.13 für $500 \mathrm{eV}, 1 \mathrm{keV}$ und $10 \mathrm{keV}$ gezeigt. $C_{11}^{\text {total }}$ wird in einem Winkelbereich zwischen $40^{\circ}$ und $85^{\circ}\left(45^{\circ}\right.$ und $85^{\circ}$ für $10 \mathrm{keV}$ ) deutlich negativ, falls den dickenabhängige Krümmungskoeffizient $D_{11}$ vernachlässigt wurde. Dieser Winkelbereich reduziert sich 


\section{Ionenstrahlinduzierte Musterbildung auf einfachen Oberflächen}

auf $67^{\circ}-85^{\circ}$, wenn der Koeffizient $D_{11}$ berücksichtigt und der Implantationskoeffizient $C_{11}^{\text {implant. }}$ vernachlässigt wird. Im Falle der Berücksichtigung der beiden Koeffizienten $D_{11}$ und $C_{11}^{\text {implant. }}$ wird $C_{11}^{\text {total }}$ in einem Winkelbereich zwischen $55^{\circ}$ uns $85^{\circ}$ eindeutig negativ und zeigt somit eine gute Übereinstimmung mit experimentellen Daten in Abbildung (4.11). Der Krümmungskoeffizient $C_{22}^{\text {total }}$ ist ähnlich wie $\mathrm{Ne}^{+}$-Bestrahlung positiv für alle Einfallswinkel und untersuchten Ionenenergien (Abbildung 4.14 und 4.15).

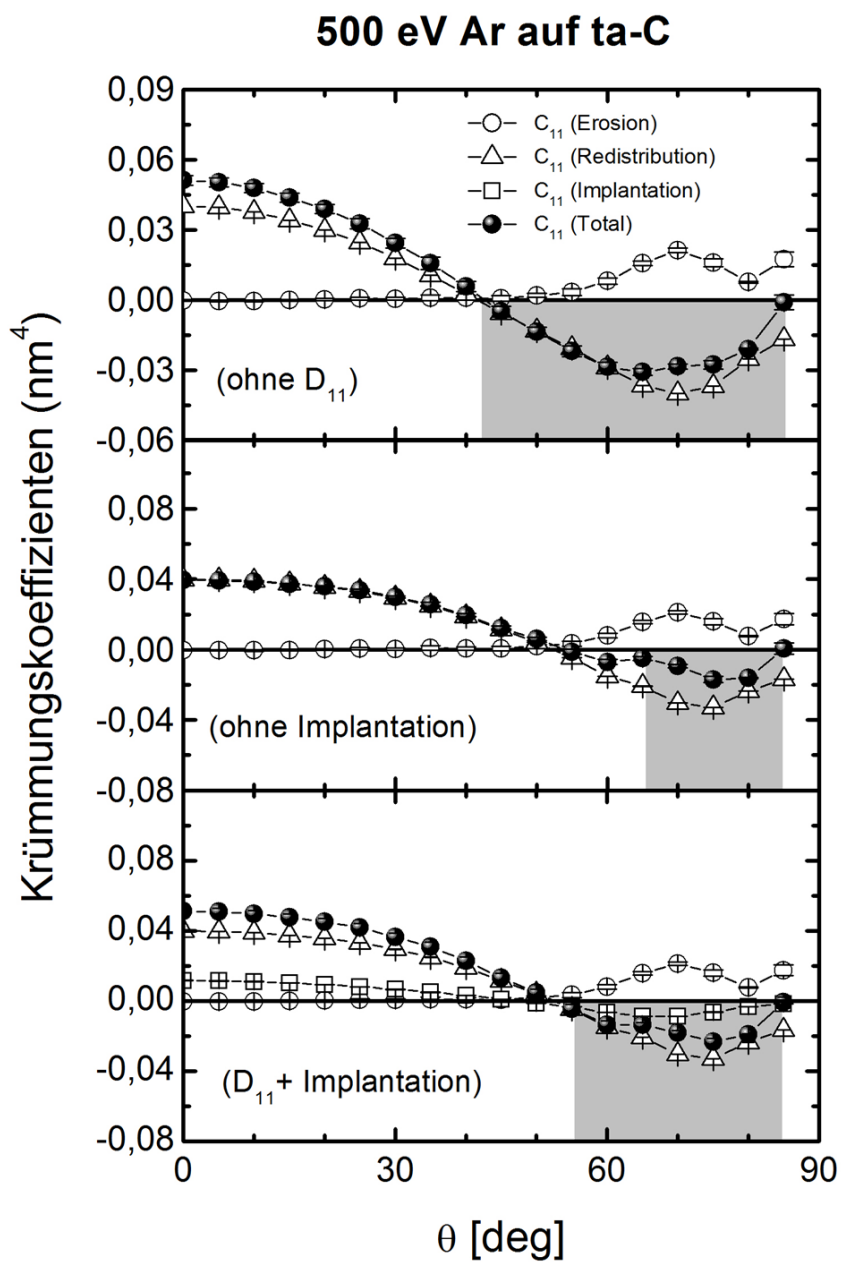

$1 \mathrm{keV}$ Ar auf ta-C

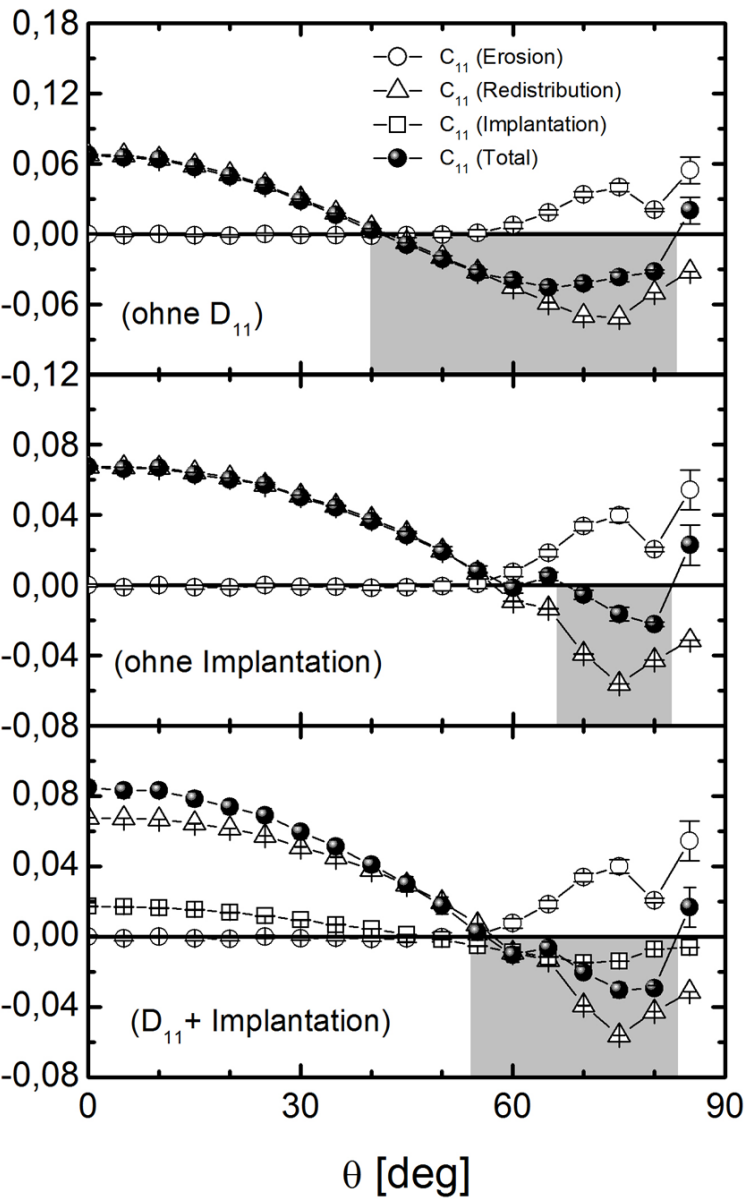

Abb. 4.12.: Berechnete Krümmungskoeffizienten $C_{11}^{\text {total }}$ für $500 \mathrm{eV}$ und $1 \mathrm{keV} \mathrm{Ar}^{+}$ Ionenbestrahlung von ta-C. Der Koeffizient $C_{11}^{\text {eros. }}$ beinhaltet die Krümmungsabhängigkeit der Erosionskraterfunktion $T_{11}$. Im oberen Diagramm beinhaltet $C_{11}^{\text {total }}$ den Effekt der Ionenimplantation $C_{11}^{\text {implant. }}$, aber die Dickenabhängigkeit der bestrahlten Schicht $D_{11}$ ist vernachlässigt. Im mittleren Diagramm beinhaltet $C_{11}^{\text {total }}$ die Dickenabhängigkeit $D_{11}$ aber der Effekt der Ionenimplantation $C_{11}^{\text {implant. }}$ ist nicht berücksichtigt. Im unteren Diagramm sind die beiden Koeffizienten $D_{11}$ und $C_{11}^{\text {implant. }}$ berücksichtigt. Der grau schattierte Bereich zeigt die Winkelbereiche an, in denen $C_{11}^{\text {total }}<0$ ist. 


\section{$10 \mathrm{keV}$ Ar auf ta-C}

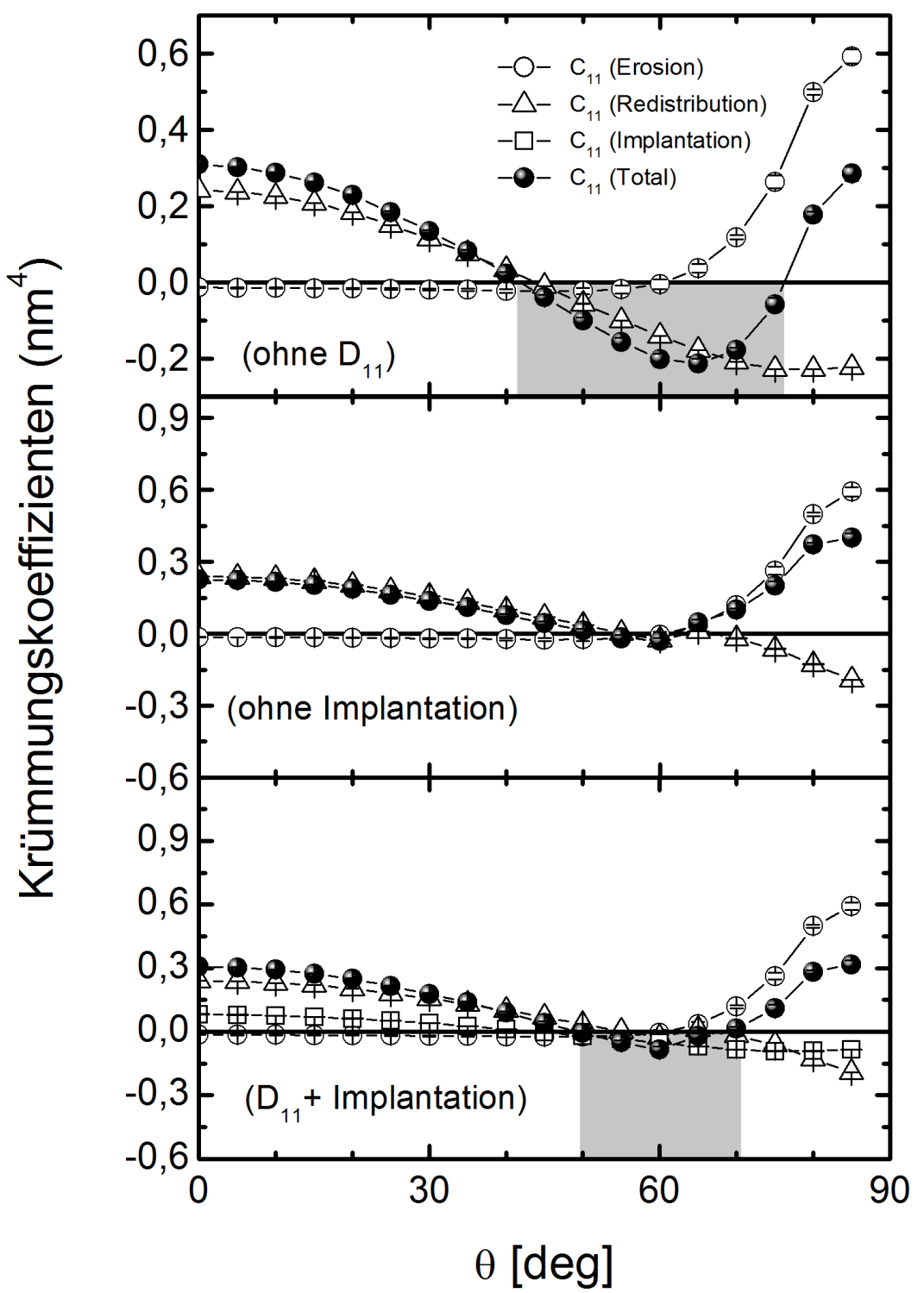

Abb. 4.13.: Berechnete Krümmungskoeffizienten $C_{11}^{\text {total }}$ für $10 \mathrm{keV} \mathrm{Ar}^{+}$-Ionenbestrahlung von ta-C. Der Erosionskoeffizient $C_{11}^{\text {eros }}$ beinhaltet die Krümmungsabhängigkeit der Erosionskraterfunktion $T_{11}$. Im oberen Diagramm beinhaltet $C_{11}^{\text {total }}$ den Effekt der Ionenimplantation $C_{11}^{\text {implant. }}$, aber die Dickenabhängigkeit der bestrahlten Schicht $D_{11}$ ist vernachlässigt. Im mittleren Diagramm beinhaltet $C_{11}^{\text {total }}$ die Dickenabhängigkeit $D_{11}$, aber der Effekt der Ionenimplantation $C_{11}^{\text {implant. }}$ ist nicht berücksichtigt. Im unteren Diagramm sind die beiden Koeffizienten $D_{11}$ und $C_{11}^{\text {implant. }}$ berücksichtigt. Der grau schattierte Bereich zeigt die Winkelbereiche an, in denen $C_{11}^{\text {total }}<0$ ist. 


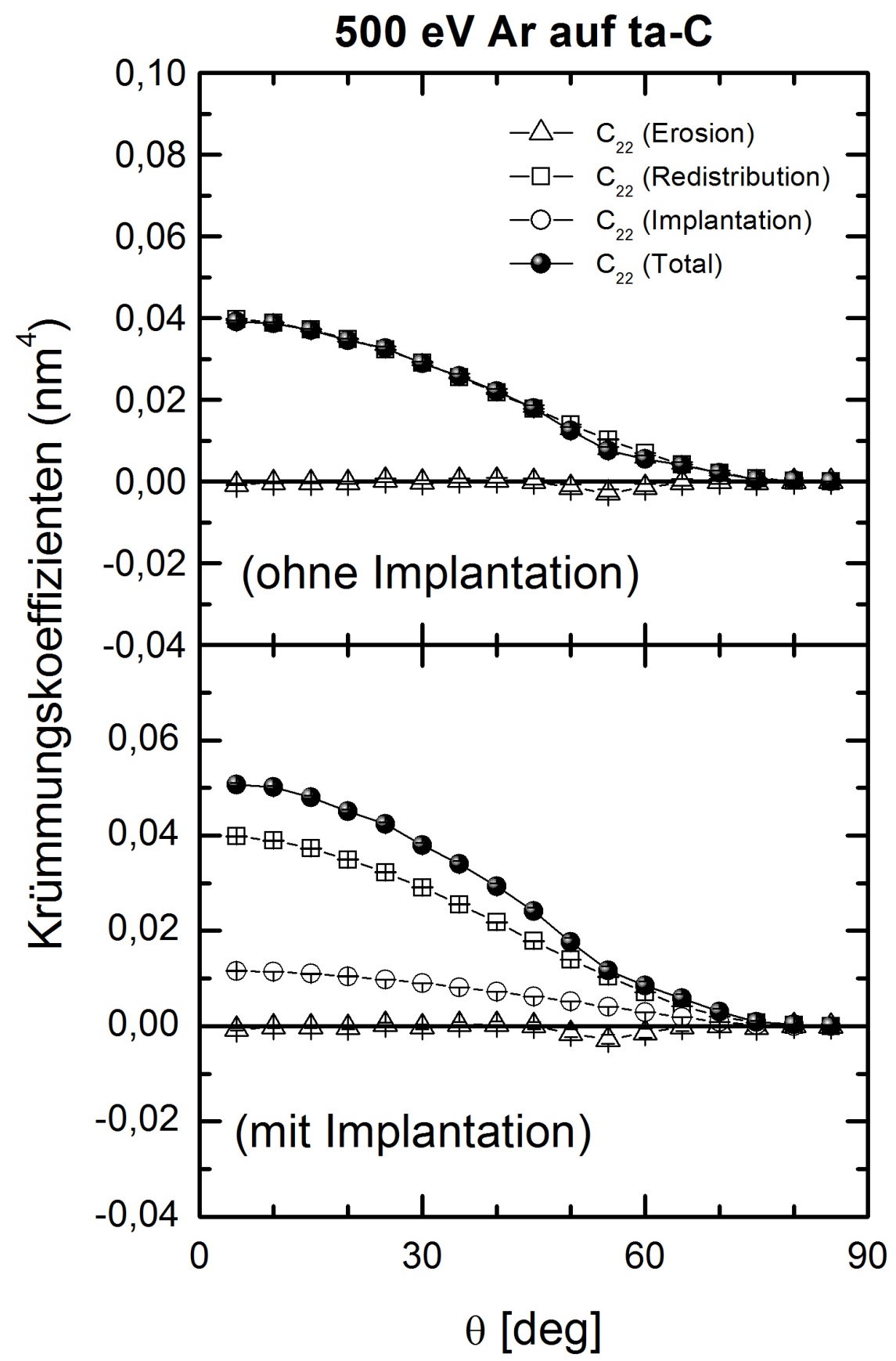

Abb. 4.14.: Berechnete Krümmungskoeffizienten $C_{22}^{\text {total }}$ für $5 \mathrm{keV}$ und $10 \mathrm{keV} \mathrm{Ne}^{+}$ Ionenbestrahlung von ta-C. Der Erosionskoeffizient $C_{22}^{\text {eros. }}$ beinhaltet die Krümmungsabhängigkeit der Erosionskraterfunktion $T_{22}$. Im oberen Diagramm beinhaltet $C_{22}^{\text {total }}$ den Effekt der Ionenimplantation $C_{22}^{\text {implant. }}$. Im unteren Diagramm wird der Effekt der Ionenimplantation nicht berücksichtigt. 

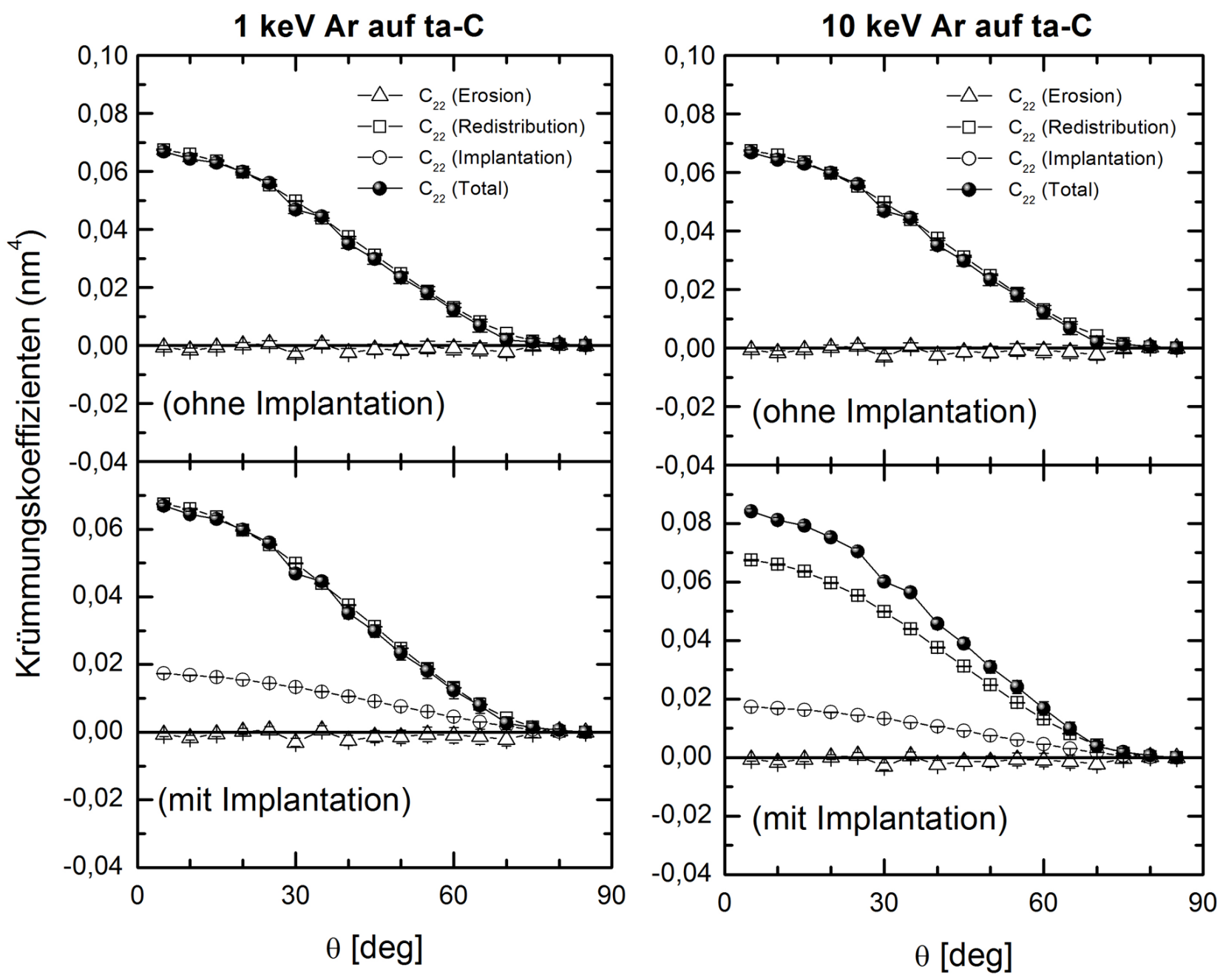

Abb. 4.15.: Berechnete Krümmungskoeffizienten $C_{22}^{\text {total }}$ für $1 \mathrm{keV}$ und $10 \mathrm{keV} \mathrm{Ar}^{+}$Ionenbestrahlung von ta-C. Der Erosionskoeffizient $C_{22}^{\text {eros. }}$ beinhaltet die Krümmungsabhängigkeit der Erosionskraterfunktion $T_{22}$. Im oberen Diagramm beinhaltet $C_{22}^{\text {total }}$ den Effekt der Ionenimplantation $C_{22}^{\text {implant. }}$. Im unteren Diagramm wird der Effekt der Ionenimplantation nicht berücksichtigt.

\subsubsection{Diskussion}

In diesem Abschnitt wurde die Rippelmusterbildung auf amorphe Kohlenstoffschichten durch $\mathrm{Ne}^{+}$- sowie $\mathrm{Ar}^{+}$-Ionenbestrahlung für einen breiten Bereich von Ionenenergien und Ioneneinfallswinkeln untersucht. Es wurde finden ein gut definiertes Regime von Bestrahlungsparametern gefunden, in dem die Bildung von parallelen Rippel auftritt. Im Fall von $\mathrm{Ne}^{+}$-Ionen auf ta-C werden Rippelmuster für Einfallswinkel zwischen $50^{\circ}$ und $75^{\circ}$ und Ionenenergien von nicht mehr als $5 \mathrm{keV}$ beobachtet. Im Gegensatz dazu bleibt die Oberfläche nach $10 \mathrm{keV} \mathrm{Ne}{ }^{+}$-Bestrahlung mit ähnlicher Ionenfluenz flach, denn die Wachstumsrate 


\section{Ionenstrahlinduzierte Musterbildung auf einfachen Oberflächen}

scheint so klein zu sein, dass auch nach einer Fluenz von $5 \times 10^{17}$ Ionen $/ \mathrm{cm}^{2}$ keine Rippelmuster beobachtet werden. Für den Fall $\mathrm{Ar}^{+}$-Ionen auf ta-C werden Rippelmuster für Einfallswinkel zwischen etwa $60^{\circ}$ und $75^{\circ}$ beobachtet. RBS-Analysen der bestrahlten Proben zeigen eine signifikante Restkonzentration an implantiertem Ne und Ar. Der Vergleich mit SDTrimSP-Simulationen zeigt, dass die implantierte Fraktion leicht Werte über 50\% übersteigen kann. Der Verlust von Ne und Ar wird dann hauptsächlich durch Sputtererosion und Rückreflexion bei größeren Einfallswinkeln bestimmt. Berechnungen der nur auf dem BH- und CV-Modell basierenden Krümmungskoeffizienten unter Verwendung der Kraterfunktionstheorie sagen eine Musterbildung für Einfallswinkel von nur $45^{\circ}$ vorher. Nur wenn die Abhängigkeit der dynamischen Schichtdicke $D_{11}$ berücksichtigt wird, kann man den Bereich, in dem parallele Rippelmuster gebildet werden, korrekt vorhersagen. Der unterschiedliche Beitrag von Erosion, Massen-Redistribution und Implantation zum Gesamtkrümmungskoeffizienten $C_{11}^{\text {total }}$ kann aus Abb. 4.3), 4.4, 4.5, 4.12) und 4.13 abgeleitet werden. Erosion hat hauptsächlich einen stabilisierenden Beitrag bei größeren Einfallswinkeln und die Musterbildung ist hauptsächlich auf eine Massen-Redistribution und Ionenimplantation zurückzuführen. Die Simulationen zeigen auch, dass bei Einfallswinkeln bis etwa $50^{\circ}$ keine Musterbildung auftritt. Die Stabilisierung der Oberfläche beruht in diesem Winkelbereich auf dem positiven Koeffizienten $D_{11}$ und auch auf dem stabilisierenden Effekt der Ne- und Ar-Implantation, d.h. dem Koeffizienten $C_{11}^{\text {implant. }}$.

\subsubsection{Fazit}

Mit Hilfe des Monte-Carlo-Simulationsprogramms SDTrimSP wurden die Krümmungskoeffizienten $C_{11}^{\text {total }}$ unter Berücksichtigung der BH-Theorie berechnet, einschließlich der von Harrison und Bradley eingeführten Krümmungsabhängigkeit der Kraterfunktion [61], des CV-Modells [28], der von Hofsäss et al. eingeführten dynamischen Schichtdickenabhängigkeit sowie der Effekt der Ionenimplantation [70, 71, 75]. Die $C_{11}$-Koeffizienten werden im experimentell beobachteten Bereich negativ und sind somit in der Lage, die Musterbildung durch Bestrahlung mit niederenergetischen $\mathrm{Ne}^{+}$- und $\mathrm{Ar}^{+}$-Ionen quantitativ vorherzusagen. Die Berechnungsergebnisse stimmen sehr gut mit der Zusammenstellung der experimentellen Ergebnisse, die in den Abb. 4.1), 4.10) und 4.11) gezeigt sind, überein. Ohne den stabilisierenden Krümmungskoeffizienten kann die Abwesenheit von Mustern bei $45^{\circ}$ nicht erklärt werden. Weiterhin findet man, dass der Implantationskoeffizient für Winkel über $45^{\circ}$ destabilisierend wirkt und insbesondere bei einer Ionenbestrahlung von $850 \mathrm{eV}$ bis $10 \mathrm{keV}$ zur Ausbildung von Rippel-Mustern beiträgt. 


\subsection{Ionenstrahlinduzierte Musterbildung auf Si Schichten}

\subsubsection{Bestrahlung mit Ne-Ionen}

Die Bildung von parallelen Rippeln für $\mathrm{Ar}^{+}$-Ionenbestrahlung auf Si im Energiebereich zwischen $250 \mathrm{eV}$ bis $140 \mathrm{keV}$ ist in der Literatur gut dokumentiert. Es wurden jedoch nur wenige Studien für leichte $\mathrm{Ne}^{+}$-Ionen auf Si durchgeführt. Carter et al. beobachteten parallele Rippel auf Si mit einer Wellenlänge von etwa $800 \mathrm{~nm}$ für $20 \mathrm{keV} \mathrm{Ne}{ }^{+}$-Ionen und $45^{\circ}$ Einfallswinkel [29]. Um den niedrigen Bereich der Ionenenergien abzudecken, wurde in dieser Arbeit Bestrahlungen von Si-Oberflächen mit $\mathrm{Ne}^{+}$-Ionen zwischen $500 \mathrm{eV}$ und $1.5 \mathrm{keV}$ durchgeführt. Die AFM-Bilder sind als Funktion des Einfallswinkels und mit einer Fluenz zwischen 1 und $4 \times 10^{17}$ Ionen $/ \mathrm{cm}^{2}$ in Abbildung (4.16) dargestellt. Für $500 \mathrm{eV}$ sind parallele Rippel-Muster deutlich zu sehen. Die Wellenlänge beträgt $126 \mathrm{~nm}$ bei $45^{\circ}$ und $90 \mathrm{~nm}$ bei $60^{\circ}$. Bei $75^{\circ}$ verschwanden die Rippel und die Rauhigkeit ist nahe derjenigen Oberfläche, der nicht bestrahlt wurde. Bei $1 \mathrm{keV}$ und $1.5 \mathrm{keV}$ bleibt die Oberfläche glatt für alle Einfallswinkel zwischen $45^{\circ}$ und $75^{\circ}$. Die Abwesenheit von Mustern bei $1 \mathrm{keV}$ und $1,5 \mathrm{keV}$ stimmt mit den zuvor veröffentlichten Daten von Vishnyakov-Carter und Ziberi et al. überein [149, 159].

\subsubsection{Simulationsergebnisse}

Abbildung 4.17) und 4.18 zeigen die Monte-Carlo-Simulation der Krümmungskoeffizienten $C_{11}^{\text {total }}$ für $500 \mathrm{eV}, 1 \mathrm{keV}$ und $1.5 \mathrm{keV} \mathrm{Ne}{ }^{+}$-Ionenbestrahlung. Die Berücksichtigung der beiden Koeffizienten $D_{11}$ und $C_{11}^{\text {implant. }}$ bei $500 \mathrm{eV}$ führt zu einem negativen Gesamtkrümmungskoeffizient $C_{11}^{\text {total }}$ mit Werten zwischen $57^{\circ}$ und $80^{\circ}$ und somit zur Destabilisierung

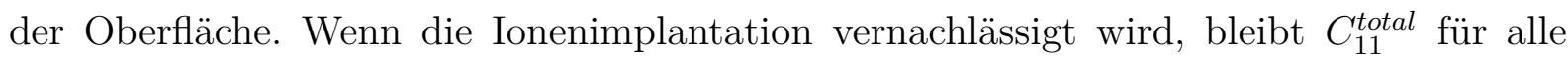
Einfallswinkel positiv. Die berechneten Krümmungskoeffizienten für $1 \mathrm{keV}$ und $1.5 \mathrm{keV}$ $\mathrm{Ne}^{+}$auf Si zeigen auch deutlich, dass $C_{11}^{\text {total }}$ für alle Einfallswinkel positiv ist, wenn die beiden Koeffizienten $D_{11}$ und $C_{11}^{\text {implant. }}$ berücksichtigt werden, sodass keine Rippelmuster auftreten sollten. Die Vernachlässigung des Koeffizients $D_{11}$ führt in den drei Fällen zu negativen $C_{11}^{\text {total }}$ im Winkelbereich zwischen $45^{\circ}$ und $75^{\circ}$. Die in Abbildungen (4.19) und 4.20 gezeigten Krümmungskoeffizienten $C_{22}^{\text {total }}$ sind immer positiv und die senkrechte Rippelmusterbildung wird unterdrückt. Denn Ionenimplantation führt auch bei der $\mathrm{Ne}^{+}-$ Ionenbestrahlung auf Si zu einer signifikanten Stabilisierung der Oberfläche gegen der senkrechten Rippelmusterbildung. 


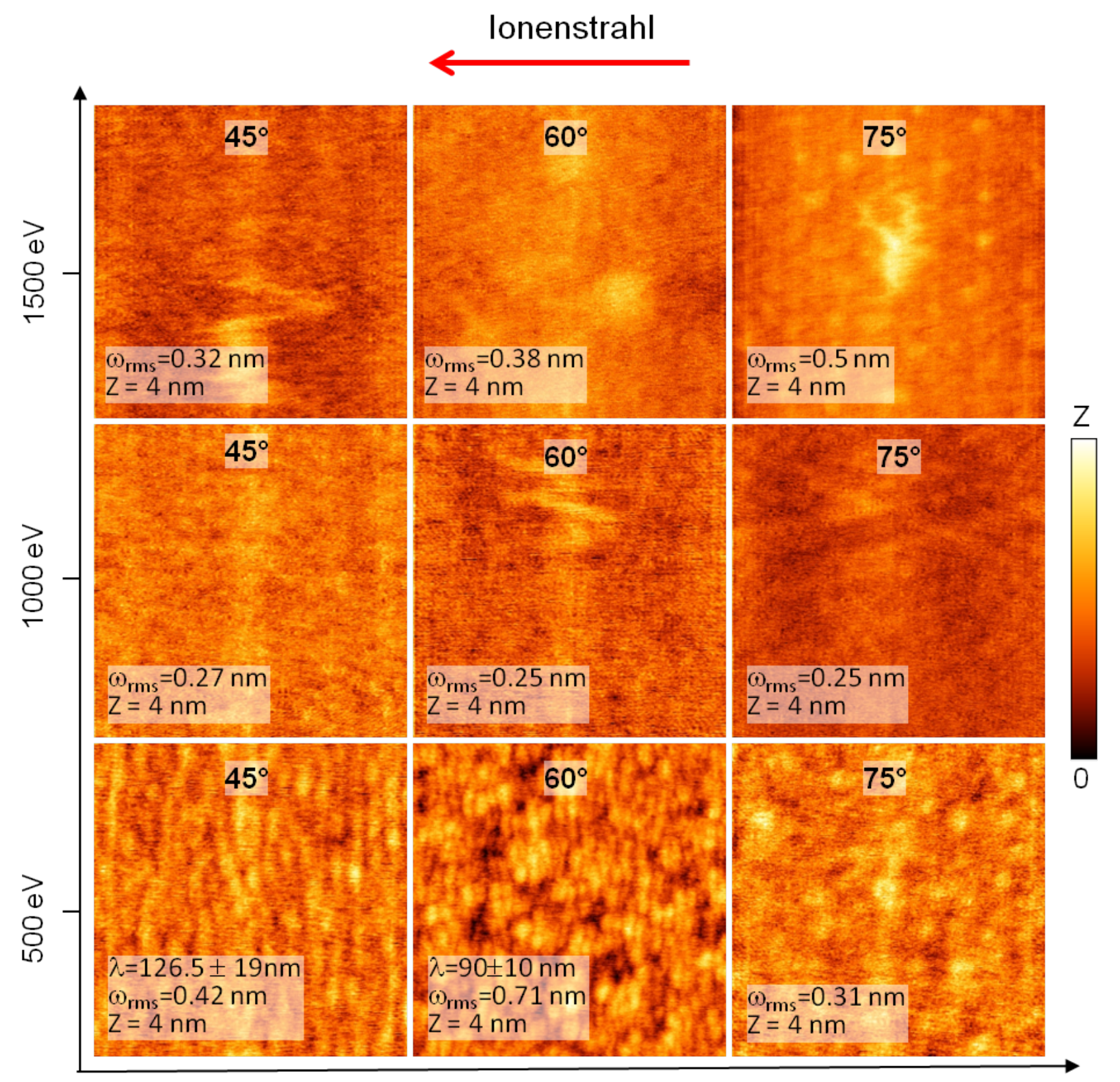

\section{Einfallswinkel}

Abb. 4.16.: AFM-Aufnahmen von den Si-Schichten nach der Bestrahlung von $500 \mathrm{eV}$, $1 \mathrm{keV}$ und $1.5 \mathrm{keV} \mathrm{Ne}{ }^{+}$-Ionen als Funktion des Einfallswinkels. Jedes Bild zeigt einen $1 \times 1 \mu \mathrm{m}^{2}$ großen Ausschnitt. 


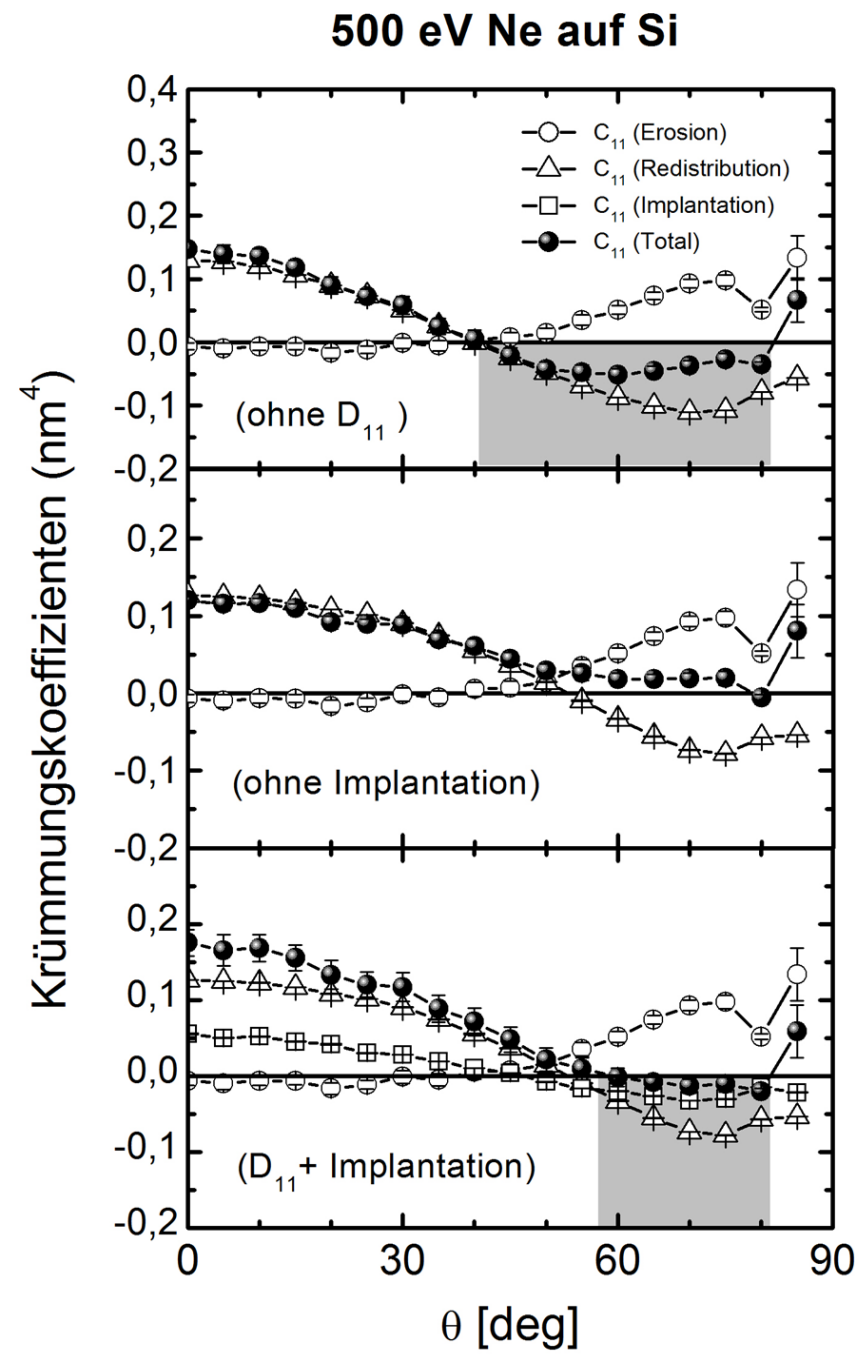

$1 \mathrm{keV} \mathrm{Ne}$ auf Si

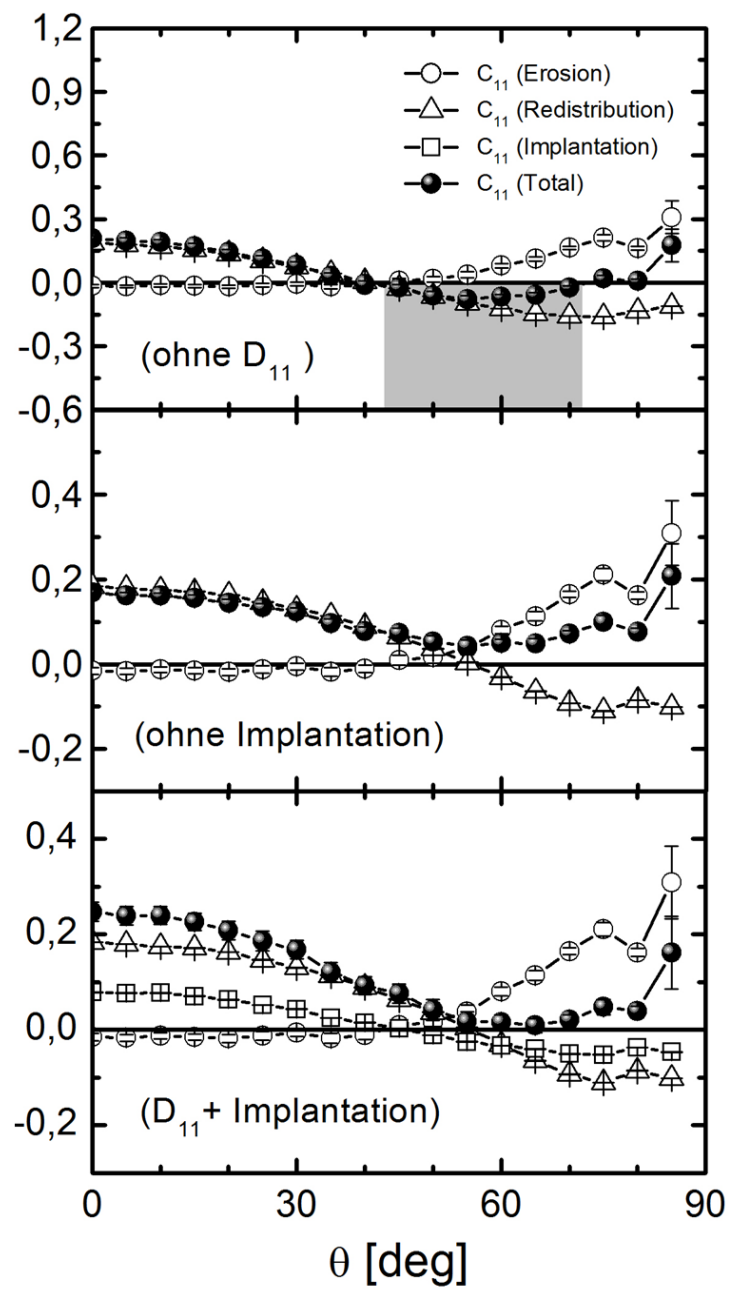

Abb. 4.17.: Berechnete Krümmungskoeffizienten $C_{11}^{\text {total }}$ für $500 \mathrm{eV}$ und $1 \mathrm{keV} \mathrm{Ne}^{+}$ Ionenbestrahlung von Si-Oberflächen. Der Erosionskoeffizient $C_{11}^{\text {eros. }}$ beinhaltet die Krümmungsabhängigkeit der Erosionskraterfunktion $T_{11}$. Im oberen Diagramm beinhaltet $C_{11}^{\text {total }}$ den Effekt der Ionenimplantation $C_{11}^{\text {implant. }}$, aber die Dickenabhängigkeit der bestrahlten Schicht $D_{11}$ ist vernachlässigt. Im mittleren Diagramm beinhaltet $C_{11}^{\text {total }}$ die Dickenabhängigkeit $D_{11}$, aber der Effekt der Ionenimplantation $C_{11}^{\text {implant. }}$ ist nicht berücksichtigt. Im unteren Diagramm sind die beiden Koeffizienten $D_{11}$ und $C_{11}^{\text {implant. }}$ berücksichtigt. Der grau schattierte Bereich zeigt die Winkelbereiche an, in denen $C_{11}^{\text {total }}<0$ ist. 


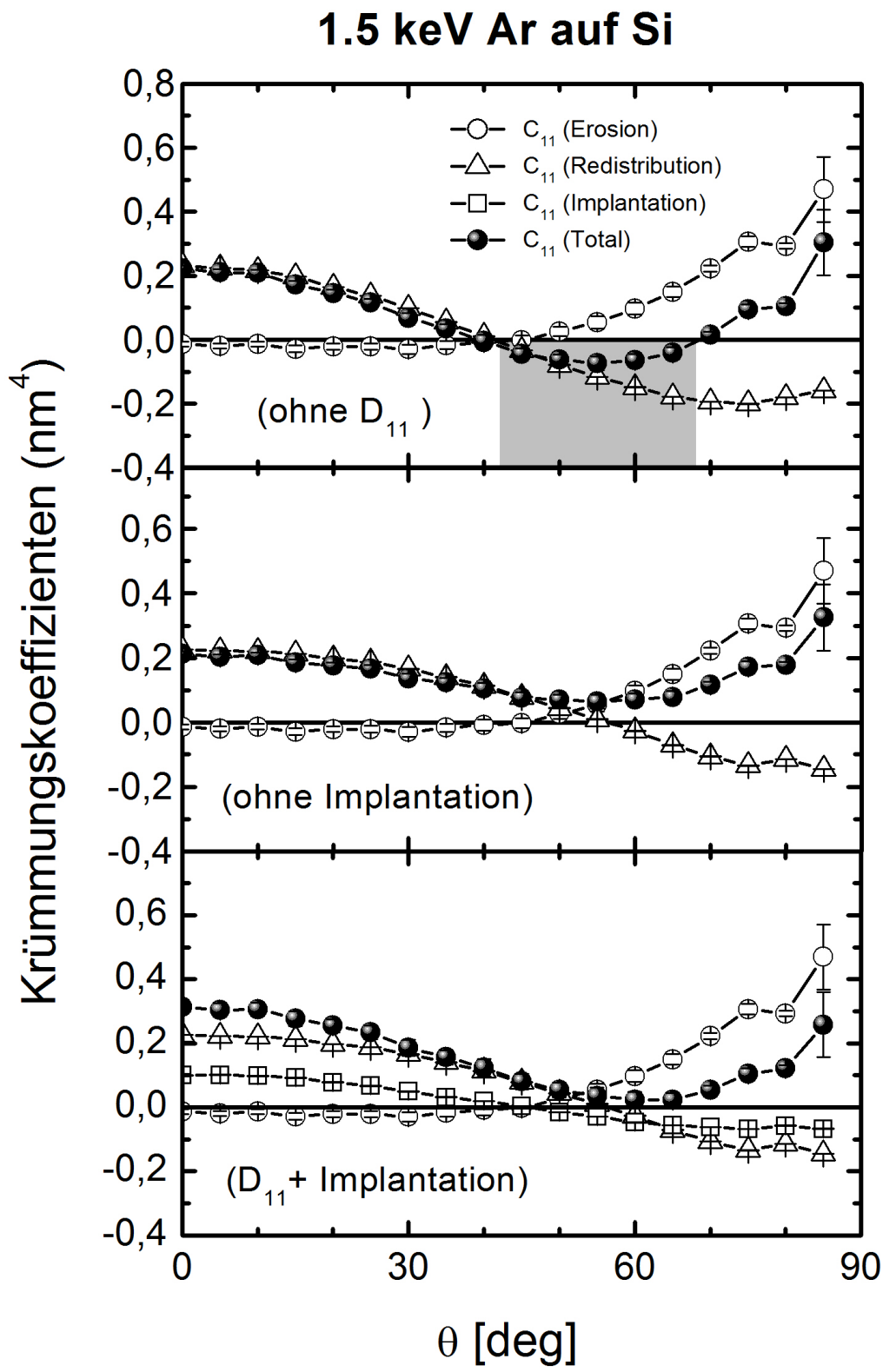

Abb. 4.18.: Berechnete Krümmungskoeffizienten $C_{11}^{\text {total }}$ für $1.5 \mathrm{keV} \mathrm{Ne}^{+}$ Ionenbestrahlung von Si-Oberflächen. Der Erosionskoeffizient $C_{11}^{\text {eros. }}$ beinhaltet die Krümmungsabhängigkeit der Erosionskraterfunktion $T_{11}$. Im oberen Diagramm beinhaltet $C_{11}^{\text {total }}$ den Effekt der Ionenimplantation $C_{11}^{\text {implant. }}$, aber die Dickenabhängigkeit der bestrahlten Schicht $D_{11}$ ist vernachlässigt. Im mittleren Diagramm beinhaltet $C_{11}^{\text {total }}$ die Dicken-

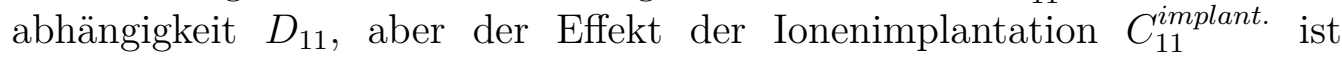
nicht berücksichtigt. Im unteren Diagramm sind die beiden Koeffizienten $D_{11}$ und $C_{11}^{\text {implant. }}$ berücksichtigt. Der grau schattierte Bereich zeigt die Winkelbereiche an, in denen $C_{11}^{\text {total }}<0$ ist. 

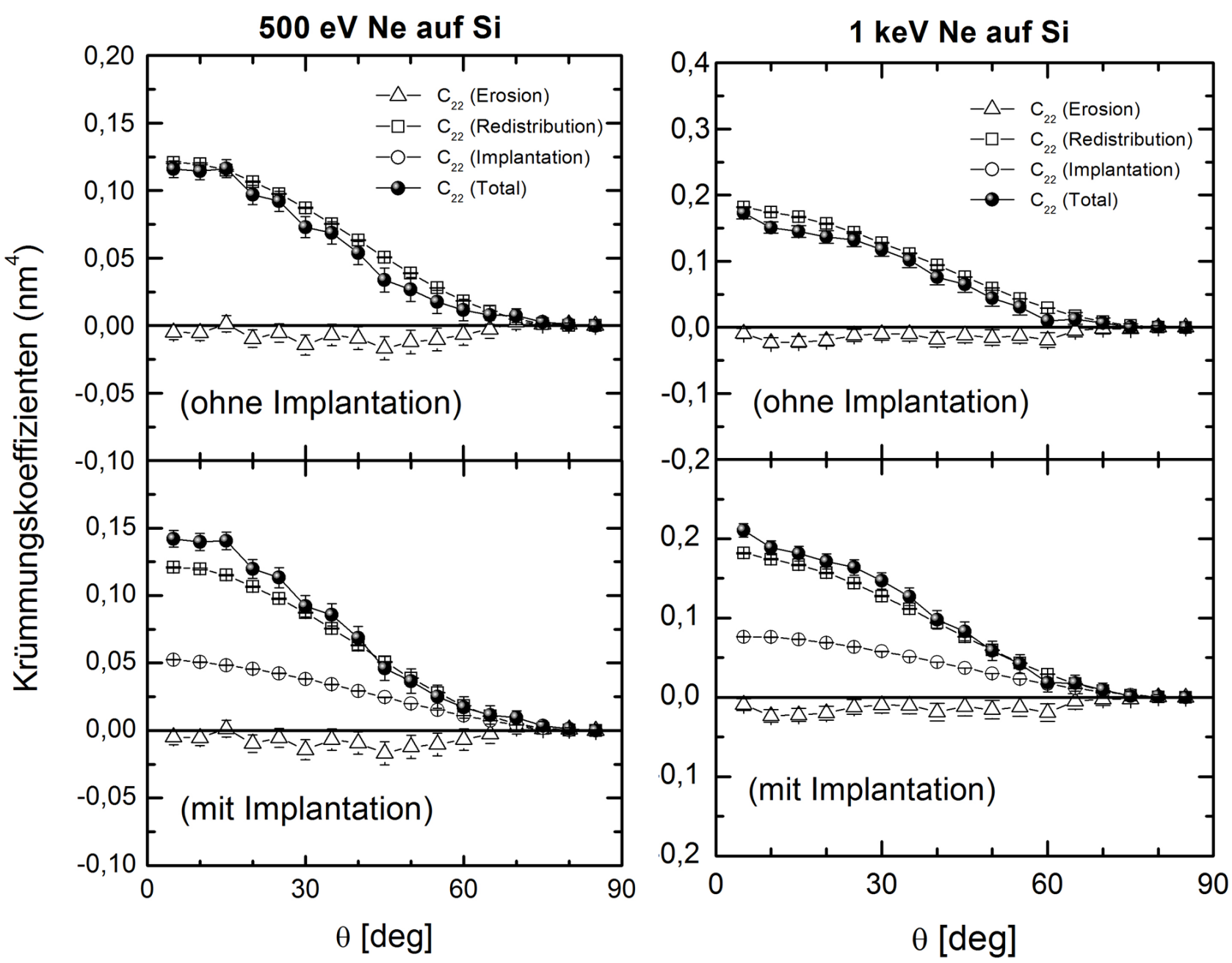

Abb. 4.19.: Berechnete Krümmungskoeffizienten $C_{22}^{\text {total }}$ für $500 \mathrm{eV}$ und $1 \mathrm{keV} \mathrm{Ne}^{+}$ Ionenbestrahlung von Si-Oberflächen. Der Erosionskoeffizient $C_{22}^{\text {eros. }}$ beinhaltet die Krümmungsabhängigkeit der Erosionskraterfunktion $T_{22}$. Im oberen Diagramm beinhaltet $C_{22}^{\text {total }}$ den Effekt der Ionenimplantation $C_{22}^{\text {implant. }}$. Im unteren Diagramm wird der Effekt der Ionenimplantation nicht berücksichtigt. 


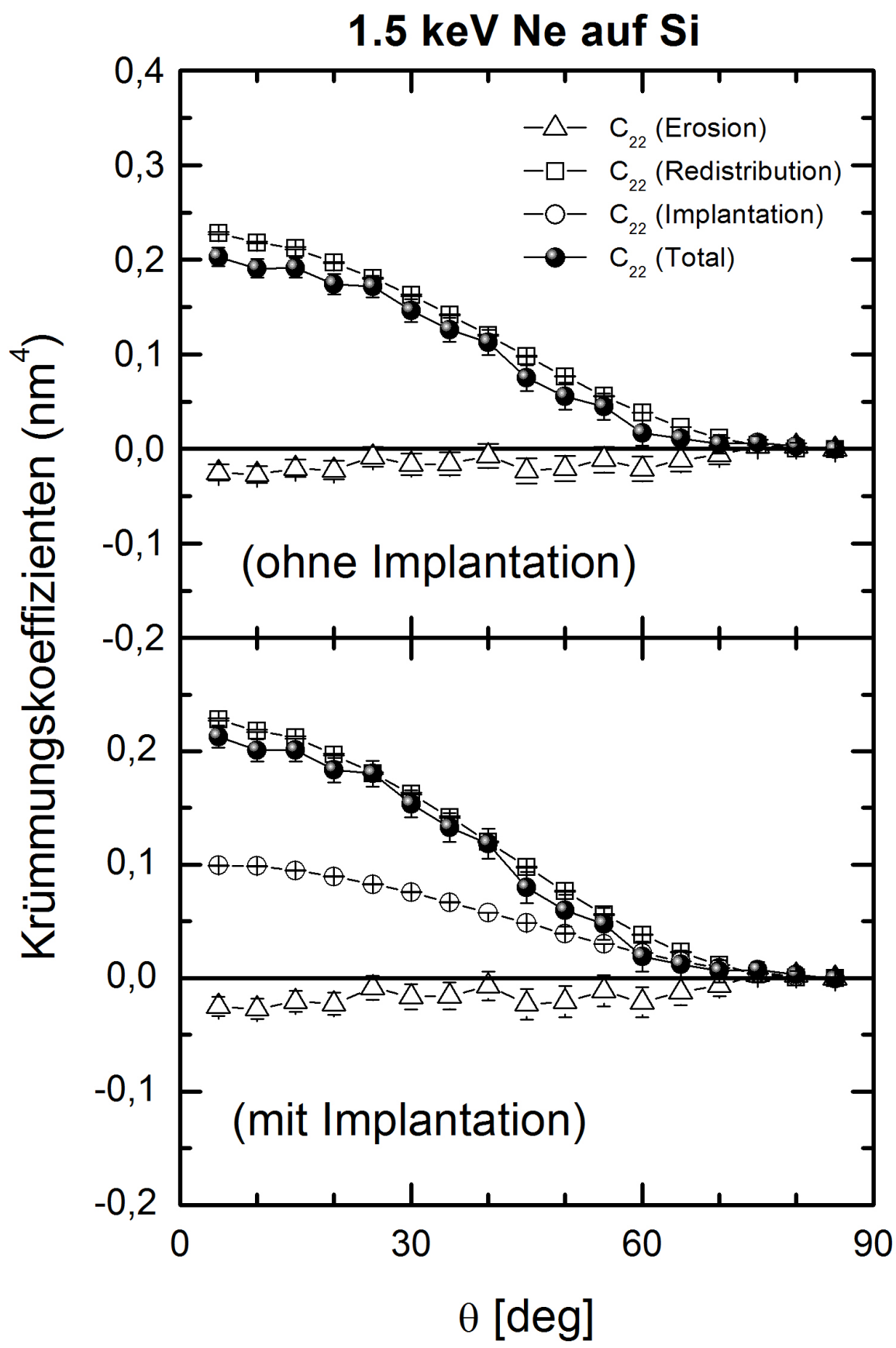

Abb. 4.20.: Berechnete Krümmungskoeffizienten $C_{22}^{\text {total }}$ für $1.5 \mathrm{keV} \mathrm{Ne}^{+}$ Ionenbestrahlung von Si-Oberflächen. Der Erosionskoeffizient $C_{22}^{\text {eros. }}$ beinhaltet die Krümmungsabhängigkeit der Erosionskraterfunktion $T_{22}$. Im oberen Diagramm beinhaltet $C_{22}^{\text {total }}$ den Effekt der Ionenimplantation $C_{22}^{\text {implant. }}$. Im unteren Diagramm wird der Effekt der Ionenimplantation nicht berücksichtigt. 


\subsubsection{Diskussion und Fazit}

In diesem Abschnitt wurden neue experimentelle Daten zur Verfügung gestellt, die zeigen, dass parallele Rippelmusterbildung induziert durch Bestrahlung mit niederenergetischen $\mathrm{Ne}^{+}$-Ionen auf Si-Oberflächen für $1 \mathrm{keV}$ und $1.5 \mathrm{keV}$ Ionenenergie gehemmt oder zumindest stark unterdrückt wird. Bei einer Energie von $500 \mathrm{eV}$ treten ausgeprägte Muster innerhalb eines Winkelbereichs zwischen etwa $45^{\circ}$ und $60^{\circ}$ auf. Bei einer hohen Energie von $20 \mathrm{keV}$ ist die Bildung von Rippelmustern in den früheren experimentellen Veröffentlichungen dokumentiert [29]. Weder die BH-Theorie (einschließlich der Krümmungsabhängigkeit der Kraterfunktion $T_{11}$ selbst) [22, 61] noch das CV-Modell [28] oder die Kombination von beiden ist in der Lage, das Fehlen von Rippel-Mustern im niedrigen Ionenenergiebereich zu erklären. Jedoch mit der Berücksichtigung der dynamischen Dickenanhängigkeit $D_{11}$ und des Implantationskoeffizients $C_{11}^{\text {implant. }}$ können wir die Abwesenheit von Rippelmuster bei $1 \mathrm{keV}$ und $1.5 \mathrm{keV} \mathrm{Ne}{ }^{+}$-Ionenbestrahlung verstehen. Der Grund für das Fehlen von Mustern liegt in diesem Fall im $T_{11^{-}}$und $D_{11^{-}}$Term. Die Krümmungsabhängigkeit der Erosionskraterfunktion beim $1 \mathrm{keV} \mathrm{Ne} \mathrm{Ne}^{+}$-Ionen auf Si verschiebt den Nulldurchgang der erosiven $C_{11}^{\text {eros. }}$-Koeffizienten von $62^{\circ}$ auf $43^{\circ}$ (Abbildung 4.21). Der stabilisierende dickeabhängige Term $C_{11}^{\text {redist. }}$ verschiebt den Nulldurchgang der redistributiven Koeffizienten von $40^{\circ}$ auf $55^{\circ}$.
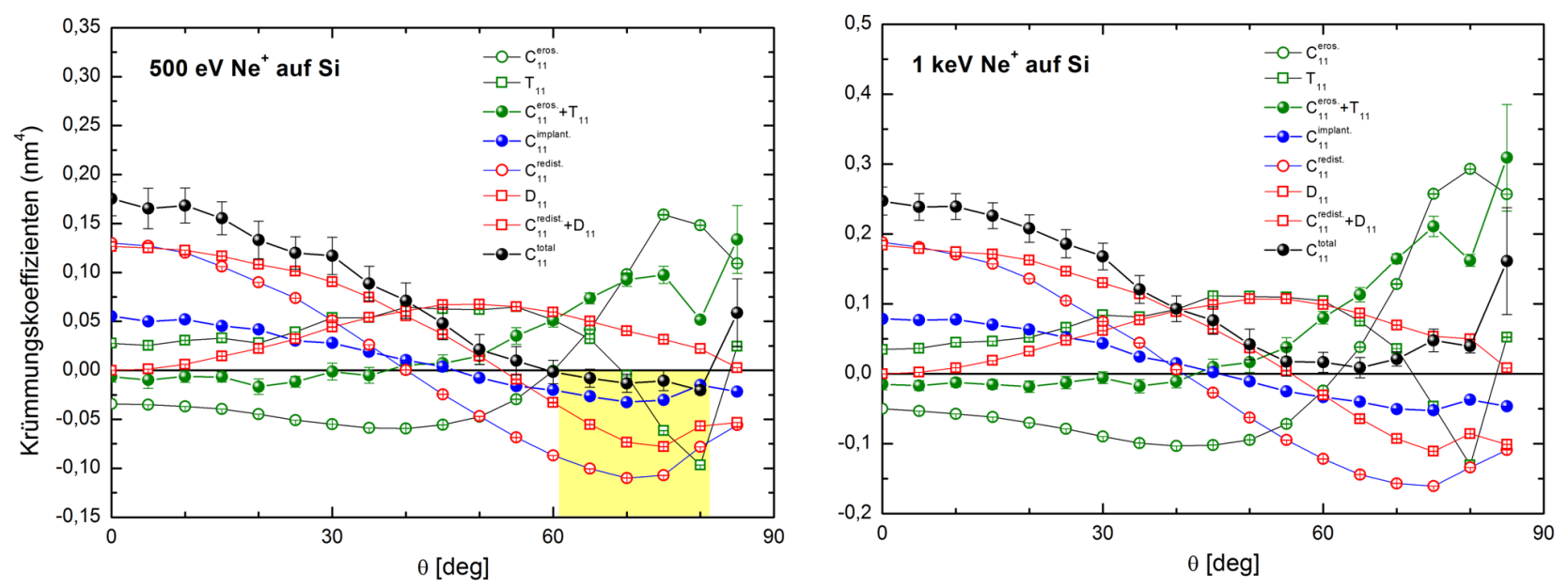

Abb. 4.21.: Simulationsergebnisse für $500 \mathrm{eV}$ und $1 \mathrm{keV} \mathrm{Ne}{ }^{+}$auf Si. Gezeigt sind die unterschiedlichen Beiträge zum Krümmungskoeffizienten $C_{11}^{\text {total }}$.

Darüber hinaus ist der Sputter-Effekt bei 500 eV schwächer und der stabilisierende Erosionskoeffizient $C_{11}^{\text {eros. }}$ bei größeren Winkeln ist kleiner und dominiert nicht über den destabilisierenden Redistributionskoeffizienten $C_{11}^{\text {redist. }}$. Die Ionenerosion blockiert die Muster- 
bildung über $55^{\circ}$, während die Mass-Redistribution Musterbildung unter $50^{\circ}$ verhindert. Infolgedessen bleibt der Gesamtkrümmungskoeffizient $C_{11}$ immer positiv oder Null.

Der Implantationskoeffizient $C_{11}^{\text {implant. }}$ ist ein destabilisierender Koeffizient ab einem Ein-

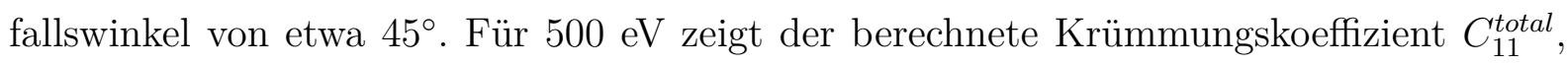
dass $C_{11}^{\text {total }}$ ohne die Ionenimplantation für alle Einfallswinkel positiv wird und keine Rippelmuster auftreten sollten. Der stabilisierende Krümmungskoeffizient $D_{11}$ ist aufgrund des letzteren Beitrags entscheidend, um eine gute Übereinstimmung zwischen theoretischer Vorhersage und experimentellen Ergebnissen zu erhalten. Bei Einfallswinkeln bis zu einem kritischen Wert von etwa $45^{\circ}$ trägt der Einbau von Ionen zur Stabilisierung der Oberfläche in der projizierten Richtung des Ionenstrahls bei. Bei größeren Einfallswinkeln ist der Beitrag destabilisierend und kann in bestimmten Fällen zur Bildung von Rippelmustern führen. 


\section{Ionenstrahlinduzierte Musterbildung auf a-C mit gleichzeitigen Kodeposition von Fremdatomen}

\subsection{Einführung}

Wir untersuchen in diesem Kapitel die Bildung von Rippelmustern auf tetraedrischen amorphen Kohlenstoffschichten (ta-C) während der Ionenstrahl-Erosion bei normalem Einfall unter gleichzeitiger Deposition verschiedener metallischer Surfactant-Atome. Hofsäss et al. [68] haben ein Musterbildungsszenario basierend auf anfänglichen chemischen Effekten und Phasenseparationsprozessen eingeführt. Nach Hofsäss et al. sollten ausgeprägte Muster für Nb-, Mo- und Hf-Surfactant mit amorphem Kohlenstoff als Substrat entstehen. Außerdem wird eine schwache Musterbildung für W- und Ti-Surfactant auf Kohlenstoff vorhergesagt [68]. Im Rahmen dieser Arbeit stellen wir neue experimentelle Daten zur ioneninduzierten Musterbildung am amorphen Kohlenstoff mit gleichzeitiger Kodeposition verschiedener Atomspezies dar. Es wurde die Musterbildung auf ta-C-Schichten für $1 \mathrm{keV} \mathrm{Xe}^{+}$- und $\mathrm{Ar}^{+}$-Ionenbestrahlung mit normalem Einfall bei Raumtemperatur und unter gleichzeitiger schräger Kodeposition von Ti, Mo, W und Pt-Surfactantatomen untersucht. Die Surfactant-Atome werden aufgrund ihres binären Phasendiagramms mit Kohlenstoff ausgewählt (Abbildung 5.1 und 5.2). Außerdem wurde den normalen Ioneneinfall gewählt, sodass die Ionenbestrahlung selbst keine Musterbildung verursacht. Ziel dieses Kapitals ist es, die Validität des von Hofsäss et al. eingeführten Musterbildungsszenarios zu verallgemeinern und nachzuweisen, dass insbesondere die Kodeposition von Metallen, die zur Karbidbildung neigen, zu ausgeprägten Punkt- und Rippel-Mustern auf ta-C-Schichten führt. 

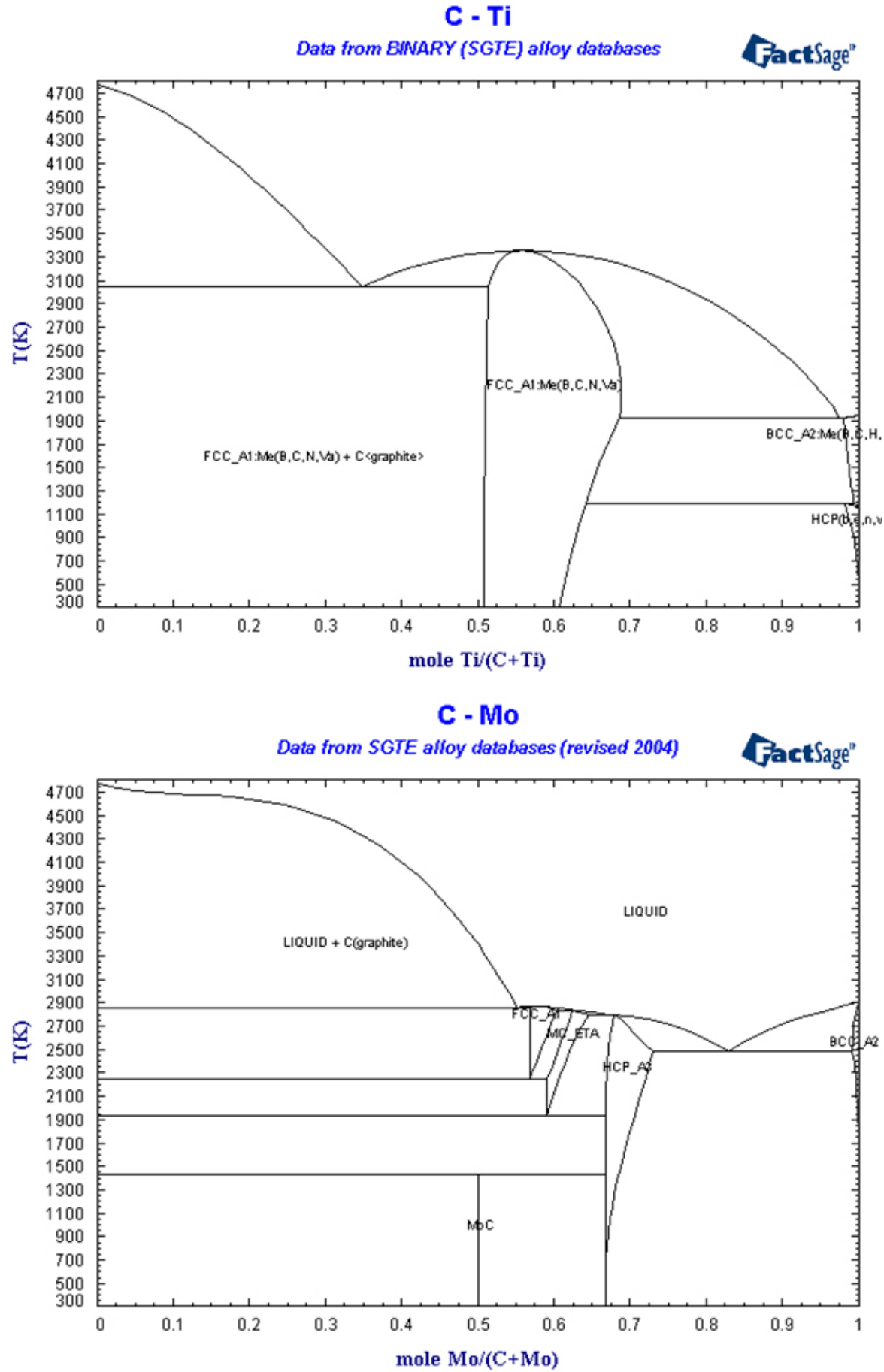

Abb. 5.1.: Phasendiagramme der Systeme C-Ti und C-Mo (Quelle: [2]) 

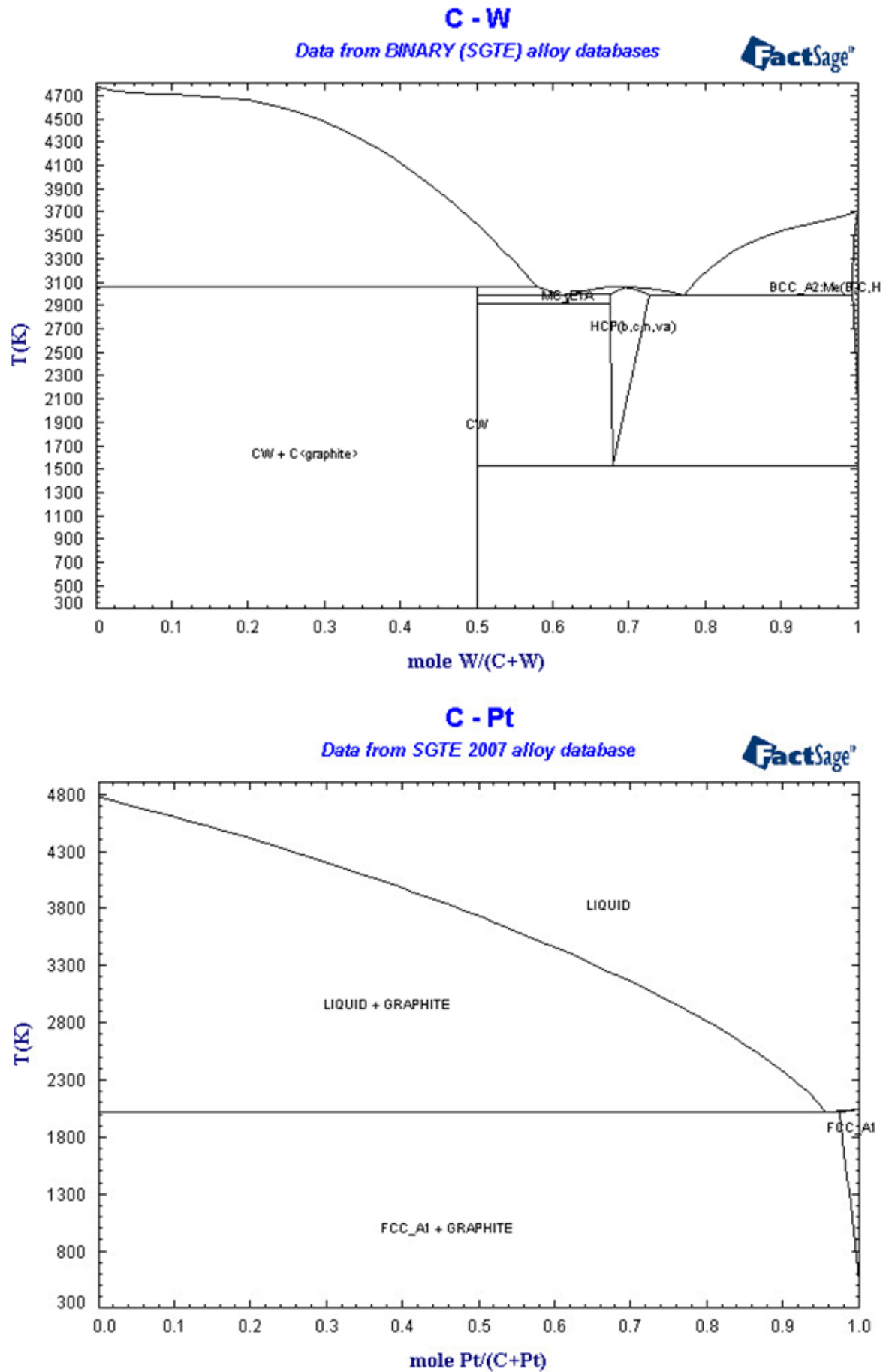

Abb. 5.2.: Phasendiagramme der Systeme W-Ti und Pt-Mo (Quelle: [2]) 


\subsection{Ergebnisse}

Tetraedrische amorphe Kohlenstoffschichten (ta-C) und benachbarte geneigte Metalltargets wurden gleichzeitig mit $1 \mathrm{keV} \mathrm{Xe} \mathrm{X}^{+}$-Ionen bei Raumtemperatur unter Hochvakuumbedingungen von $2 \times 10^{-7} \mathrm{mBar}$ bestrahlt. Der Ionenstrahl fällt entlang der normalen Richtung der ta-C-Oberfläche ein. Die Metalltargets für die gemeinsame Kodeposition sind hochreine Folien ( $\mathrm{Ti}, \mathrm{Mo}, \mathrm{W}$ und $\mathrm{Pt}$ ), die in einem Neigungswinkel von $60^{\circ}$ neben der ta-C-Probe angeordnet waren. Die Hälfte des Ionenstrahls bestrahlte die ta-C-Probe, während die andere Hälfte gleichzeitig die Metallfolie bestrahlt. Gesputterte Metallatome werden mit einer Einfallswinkelverteilung von $30^{\circ}-90^{\circ}$ zur Oberflächennormalen auf das Substrat deponiert. Die lokale Flächendichte der deponierten Metalldünnschicht kann leicht mit Rutherford-Rückstreuspektroskopie an verschiedenen Punkten $x_{i}$ auf die taC-Probe gemessen werden (siehe Abbildung 5.3). Der Metall-Depositionsfluss über die ta-C-Schicht nimmt mit zunehmender Entfernung von dem Sputtertarget ab. Auf diese Weise wird ein Gradient in der stationären Metallabdeckung hergestellt und ermöglicht es, die Erosionseffekte als Funktion der Metallabdeckung zu untersuchen.
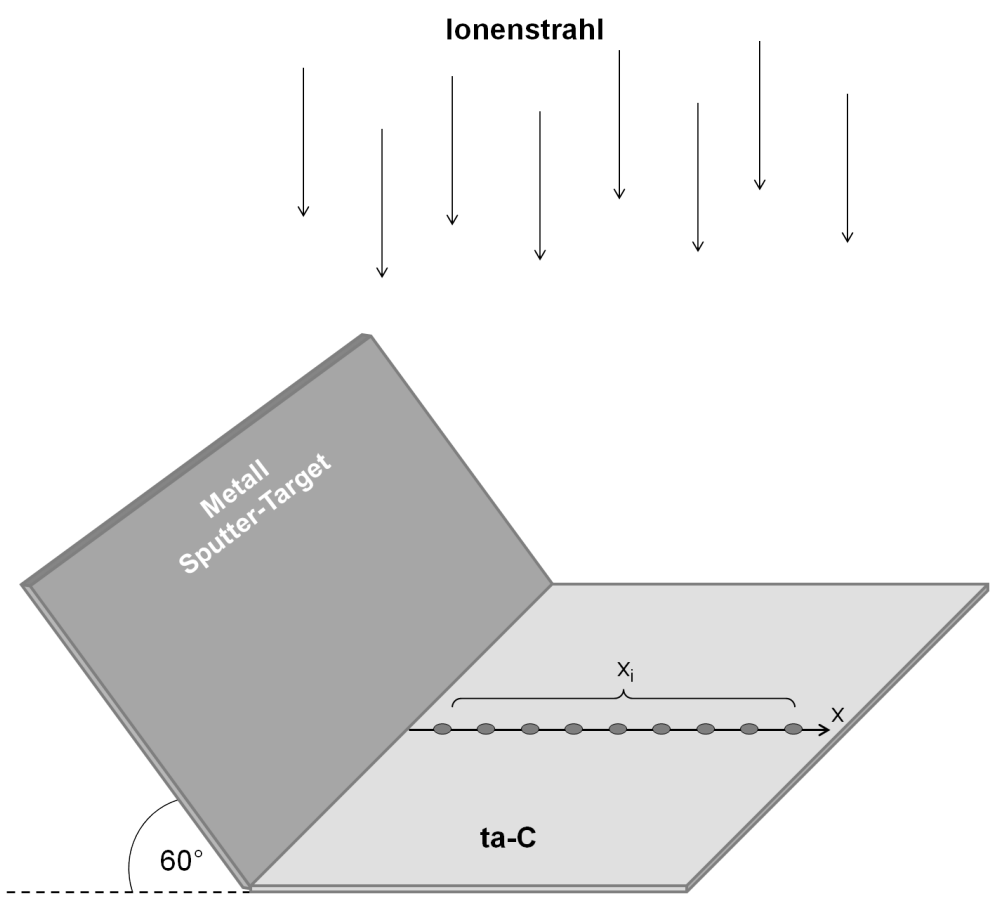

Abb. 5.3.: Schematische Darstellung des Versuchsaufbaus. ta-C-Schicht wird mit 1 $\mathrm{keV} \mathrm{Xe}^{+}$- oder $\mathrm{Ar}^{+}$-Ionen bei normalem Ioneneinfall bestrahlt. MetallKodeposition stammt von benachbarten Metallfolien-Targets, die um $60^{\circ}$ geneigt sind. Die Positionen $x_{i}$ zeigen unterschiedliche Analysepositionen für AFM- und RBS-Messungen an. 
Abbildung (5.3) zeigt eine Zusammenstellung von AFM-Bildern von Oberflächenmustern, die entweder für die Ti-, Mo-, W- oder Pt-Kodeposition und eine Fluenz von $10^{18} / \mathrm{cm}^{2}$ erhalten wurden. Für jede Metallspezies sind drei Bilder mit unterschiedlicher Oberflächenbedeckung im Bereich von $3 \times 10^{15}$ bis $8 \times 10^{15}$ Atome $/ \mathrm{cm}^{2}$ gezeigt. Ausgeprägte Rippelmuster werden in allen Fällen beobachtet, mit Ausnahme der Pt-Kodeposition. Der Mustertyp hängt von der Oberflächenbedeckung ab. Eine Erhöhung der Metallabdeckung von $4.5 \times 10^{15}$ auf $4.9 \times 10^{15}$ Atome $/ \mathrm{cm}^{2}$ ändert die Oberfläche von einer Flachheit zu einer Punktkette für Ti-Surfactant. Für Mo-Surfactan erfolgt der Übergang bei etwas geringerer Abdeckung von $4.1 \times 10^{15}$ Atome $/ \mathrm{cm}^{2}$ und entwickelt sich von einer Punktkette $\mathrm{zu}$ einem ausgeprägten Rippelmuster bei höherer Mo-Bedeckung von $8 \times 10^{15}$ Atome $/ \mathrm{cm}^{2}$. Die Musterbildung auf ta-C mit W-Surfactant beginnt bei einer ziemlich hohen Abdeckung von etwa $6.5 \times 10^{15}$ Atome $/ \mathrm{cm}^{2}$ und führt unmittelbar zu einem schwachen Rippelmuster anstelle von Punktketten. Ionenstrahlerosion mit gleichzeitiger Kodeposition von Pt-Surfactant-Atomen erzeugt keine Muster. Selbst für eine hohe Abdeckung mit PtAtomen von $6 \times 10^{15}$ Atome $/ \mathrm{cm}^{2}$ bleibt die Oberfläche extrem flach mit einer mittleren Rauhigkeit von $0.3 \mathrm{~nm}$, vergleichbar mit unbestrahlten Proben. Die Mikrostruktur der Proben wurde mittels Transmissionselektronenmikroskopie (TEM) unter Verwendung eines 200 keV Phillips CM 200-UT Mikroskops analysiert. TEM-Proben wurden mit einem fokussierten Ionenstrahlsystem (FIB) hergestellt. Diese Technik erfordert die Deposition einer Pt-Deckschicht auf der Probenoberfläche. Um die ta-C-Metalloberfläche räumlich von der Pt-Schicht in den TEM-Bildern zu trennen, haben wir nach der Ionenstrahlerosion einige Nanometer ta-C deponiert, sodass die Surfactant-Schicht als vergrabene Schicht in den TEM-Bildern erscheint. Die ta-C-Deckschicht wurde durch den Ionenbeschleuniger ADONIS (siehe Abschnitt 3.2.3) deponiert. Abbildung (5.5) zeigt Querschnitt-TEM-

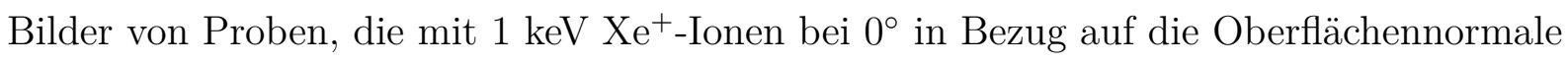
erodiert wurden. Amorphe dünne Schichten aus $\mathrm{Mo}+\mathrm{C}, \mathrm{W}+\mathrm{C}$ und $\mathrm{Ti}+\mathrm{C}$ sind deutlich als dunkle Region zu sehen. Die Bilder sind ein Schnitt entlang der Rippel-Richtung. Die $\mathrm{Mo}+\mathrm{C}-$ und $\mathrm{W}+\mathrm{C}-\mathrm{Schicht}$ sind etwa 3-4 nm dick, entsprechend der Reichweite der Mound W-Atome, die durch $1 \mathrm{keV} \mathrm{Xe}{ }^{+}$-Ionen zurückgestoßen werden. Die Ti-Schichtdicke ist ebenfalls in Übereinstimmung mit der Reichweite der zurückgestoßenen Ti+C-Atomen durch $1 \mathrm{keV} \mathrm{Ar}{ }^{+}$-Ionenbestrahlung. Nach einer früheren Studie über ta-C-Schichten mit der Surfactant-Sputter-Technik, führen Ti-Surfactants zur Bildung von NanokristallinenDünnschicht auf ta-C [69]. Jedoch konnte hier eine nanokristalline-Schicht in den TEMBilder, eventuell wegen der extremen Dünnheit der Mischschicht (3-4 nm), nicht beobachtet werden (Abbildung 5.5.). 


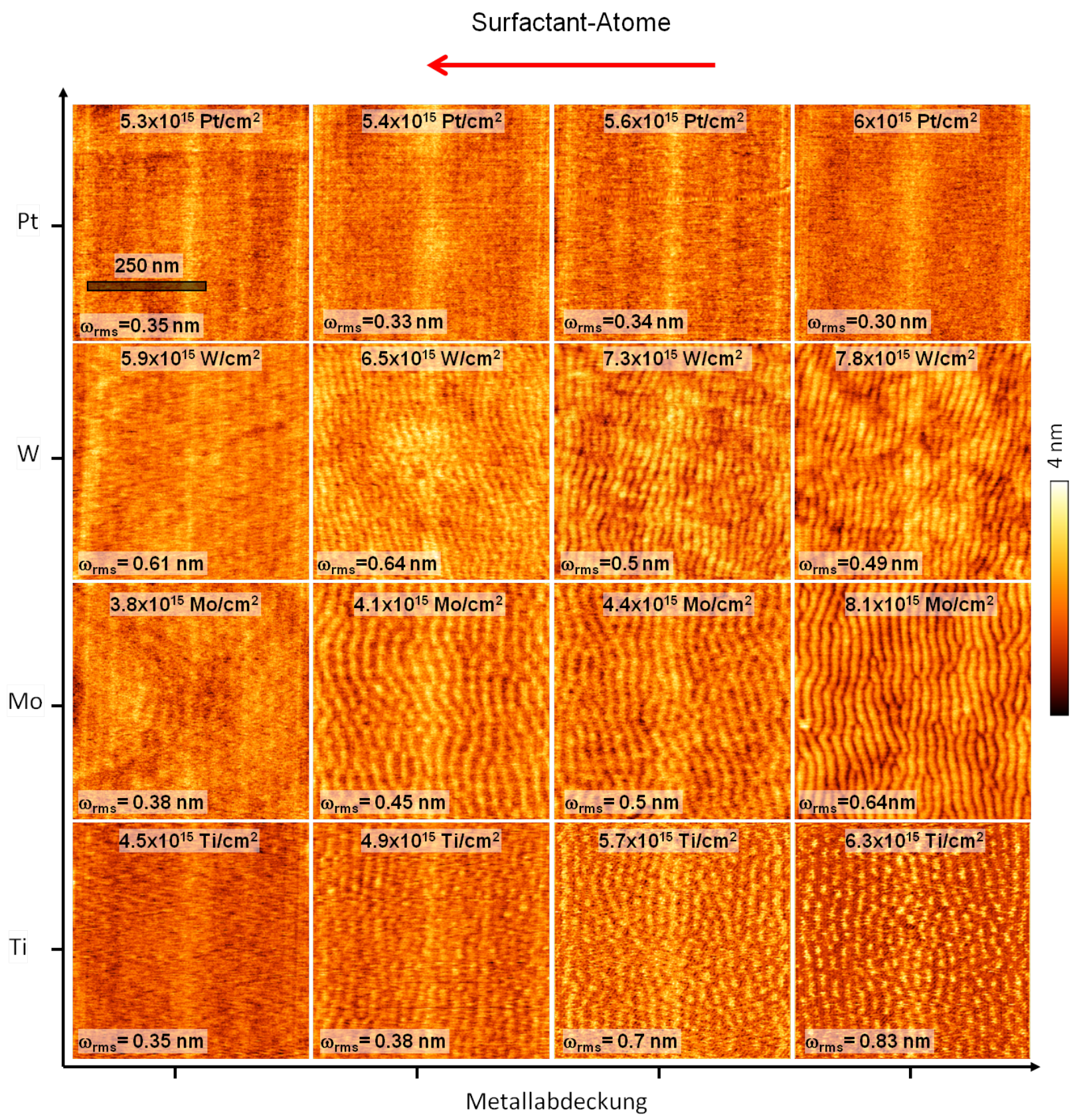

Abb. 5.4.: Zusammenstellung von AFM-Bildern von Surfactant-induzierten Mustern auf ta-C für verschiedene Metall-Surfactant-Atome (Ti, Mo, W, Pt) und unterschiedliche Metallabdeckung. Die Bedeckung in Atomeinheiten wurde mit

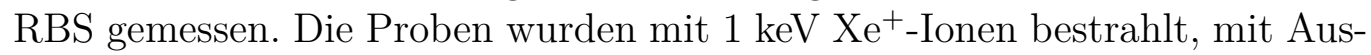
nahme von Ti-Surfactant, die Probe wurde mit $\mathrm{Ar}^{+}$-Ionen bestrahlt. Die Metall-Kodepostion erfolgte von der linken Seite der gezeigten Bilder. 


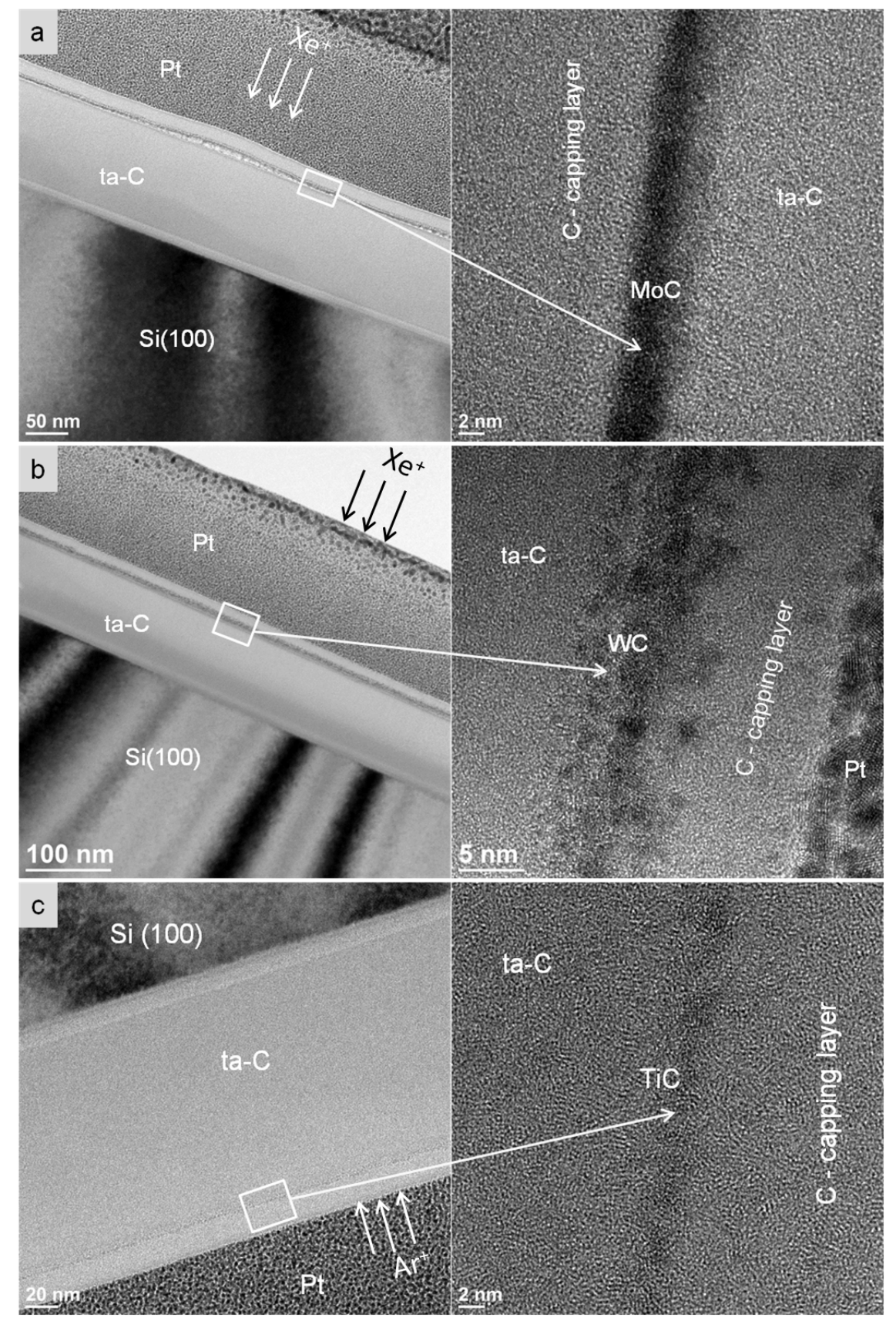

$\boldsymbol{A} \boldsymbol{b} \boldsymbol{b}$. 5.5.: Hochauflösende TEM-Analyse des Rippel-Profils. Vor der TEM-Analyse wurde eine amorphe Kohlenstoffschicht auf der Oberfläche deponiert um den Kontrast zwischen der Metall-taC-Schicht und dem Pt-C-Kleber sicherzustellen. Die Ionenstrahlrichtungen sind als Pfeile angezeigt. a) Querschnitts-TEMBild einer ta-C-Probe, bestrahlt mit $1 \mathrm{keV} \mathrm{Xe}{ }^{+}$-Ionen und Mo-Surfactant. b) Querschnitts-TEM-Bild einer ta-C-Probe, bestrahlt mit $1 \mathrm{keV} \mathrm{Xe}{ }^{+}-$Ionen und W-Surfactant. c) Querschnitts-TEM-Bild einer ta-C-Probe, bestrahlt mit 1 $\mathrm{keV} \mathrm{Ar}^{+}$-Ionen und Ti-Surfactant. 


\subsection{Diskussion und Fazit}

Die Entwicklung von Rippelmustern als Funktion der stationären Metallabdeckung und insbesondere der scharfe Übergang von flachen Oberflächen zu Rippelmustern, wenn die Metallabdeckung die Stöchiometrie $\mathrm{MeSi}_{2}$ erreicht, waren starke Argumente für die Rolle der Silizidbildung in den Metall-Si-Systemen [68. In diesem Abschnitt wurden die früheren Studien über die metall-surfactant-induzierte Musterbildung auf Si während der Ionenbestrahlung mit normalem Einfall erweitert, und zwar auf eine Reihe verschiedener Kodeposition von Metallatome auf ta-C-Oberflächen. Um die verschiedenen vorgestellten Experimente hinsichtlich chemischer Effekte, vor allem Phasenseparation, zu diskutieren, wurde die mittlere Metallatomkonzentration $c_{M}$ in der gemischten Oberflächenschicht aus der gemessenen Metallabdeckung in Analogie zu [68, 154] berechnet.

$$
c_{M}[\text { at. } \%]=100 \cdot \frac{1}{\frac{\rho_{S}}{\sigma_{M}} \cdot \frac{N_{A}}{A_{S}} \cdot d-\frac{\rho_{S} A_{M}}{\rho_{M} A_{S}}+1} .
$$

Hier sind $\rho_{S}$ und $\rho_{M}$ die Massendichten von Substrat und Metalltarget, $\mathrm{A}_{S}$ und $\mathrm{A}_{M}$ sind jeweils das Molgewicht und $\mathrm{N}_{A}$ ist Avogadro's Konstante. $d$ ist die Dicke der gemichten Schichten (Metall+C). Für die Systeme Ti-C, Mo-C und W-C existieren stabile Carbidphasen mit einem Metallgehalt von etwa $50 \%$. Für Pt-C existiert kein stabiles Karbid wie das Phasendiagramm in Abbildung 5.2 zeigt. Ohne gleichzeitige Kodepostion von Surfactant-Atomen oder bei Abwesenheit einer Kontamination der Oberfläche werden ab-

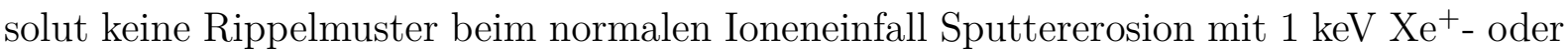
$\mathrm{Ar}^{+}$-Ionen gebildet und nur flache Oberflächen beobachtet (Abbildung 5.6).

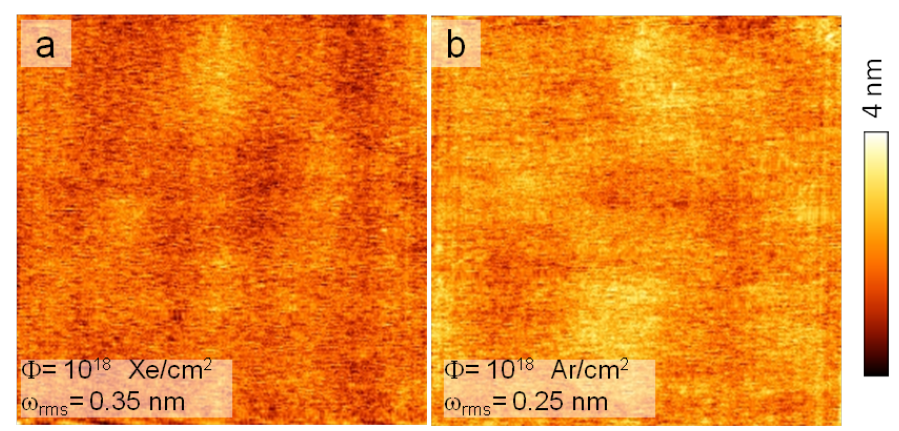

Abb. 5.6.: AFM-topographische Bilder von ta-C-Oberflächen, die mit $1 \mathrm{keV} \mathrm{Ar}^{+}$- und $\mathrm{Xe}^{+}$-Ionen bei normalem Ioneneinfall und einer Fluenz von $10^{18} / \mathrm{cm}^{2}$ erodiert wurden. Die Oberflächen sind extrem glatt mit einer rms Rauigkeit von 0.3 $\mathrm{nm}$. 
Im Falle von Systemen, die Monokarbidphasen bilden (z.B. TiC, MoC, oder WC), wird ausgeprägte selbst-organisierte Musterbildung durch kontinuierliche Metall-Kodeposition von einem benachbarten Metall-Sputtertarget, das mit dem gleichen Ionenstrahl bestrahlt wird, ausgelöst und angetrieben. Im Gegensatz dazu, bei einer Kodeposition von Elemente, die keine stabilen Karbidphasen bilden (z.B. Pt), bleibt die Oberfläche flach. Außerdem korreliert die Orientierung von Rippelmustern, die durch Metall-Kodeposition induziert werden, mit der Einfallsrichtung von kodeponierten Atomen, wie in der Literatur gezeigt wurde [68, 91, 154, 157]. Der Wellenvektor der Rippelmuster zeigt entgegengesetzt zur projizierten Richtung der einfallenden kodeponierten Atome. Anhand der Ergebnisse dieser Arbeit wird die Rolle der Phasenseparation auf Musterbildung bestätigt, worauf die Geltung des von Hofsäss et al. 68] eingeführten Musterbildungsszenarios verallgemeinert werden kann. 



\section{Zusammenfassung}

Im Rahmen dieser Arbeit wurde die ionenstrahlinduzierte Musterbildung auf Siliziumund amorphe Kohlenstoffschichten während der Bestrahlung mit niederenergetischer $\mathrm{Ne}^{+}$ und $\mathrm{Ar}^{+}$-Ionen untersucht. Ziel war es, die Qualität der vorhandenen theoretischen Modelle und deren kürzlich vorgeschlagenen Erweiterungen experimentell zu überprüfen und eine quantitative Vorhersage der Musterbildung zu ermöglichen. Mit Hilfe der MonteCarlo-Simulationen der Ion-Festkörper-Wechselwirkung war es möglich, die Momente von Erosions-, Redistributions- sowie Implantationskraterfunktion zu berechnen und somit die Krümmungskoeffizienten $\left(C_{11}\right.$ und $\left.C_{22}\right)$ in der Bewegungsgleichung der Oberfläche zu bestimmen. Aus den Krümmungskoeffizienten $C_{11}$ und $C_{22}$ können quantitative Vorhersagen über die Stabilität oder Instabilität einer Oberfläche, Wachstumsrate sowie Wellenlängen gewonnen werden. Mit dem Monte Carlo Simulationsprogramm SDTrimSP wurden die Krümmungskoeffizienten $C_{11}$ unter Berücksichtigung der BH-Theorie einschließlich der von Harrision und Bradley eingeführten Krümmungsabhängigkeit der Kraterfunktion $T_{11}$ und $T_{22}$ berechnet [61]. Außerdem wurde in den Berechnungen das CV-Modell [28], die von Hofsäss et al. eingeführte dynamische Dickenabhängigkeit der bestrahlten Schicht sowie der Effekt der Ionenimplantation berücksichtigt [70, 75].

Der erste Teil der vorliegenden Arbeit beschäftigte sich mit der Musterbildung auf tetraedrischen wasserstofffreien amorphen Kohlenstoff-Schichten (ta-C) unter niederenergetischer $\mathrm{Ne}^{+}$- und $\mathrm{Ar}^{+}$-Ionenbestrahlung. Die Ionenstrahlexperimente wurden im Ultrahochvakuum und bei Raumtemperatur durchgeführt. Im Fall von $\mathrm{Ne}^{+}$-Ionen auf ta-C werden Rippel für Einfallswinkel zwischen $50^{\circ}$ und $75^{\circ}$ und eine Ionenenergie von nicht mehr als $5 \mathrm{keV}$ beobachtet. Berechnungen der Krümmungskoeffizienten basierend auf dem BH- und CV-Modell unter Verwendung der Kraterfunktionstheorie sagen eine Musterbildung für Einfallswinkel von nur $45^{\circ}$ vorher. Nur wenn die dynamische Dickenabhängigkeit $D_{11}$ berücksichtigt wird, kann das Regime, in dem parallele Rippelmuster gebildet werden, korrekt vorhergesagt werden. Für $\mathrm{Ar}^{+}$-Ionenbestrahlung werden Rippelmuster für Einfallswinkel zwischen etwa $60^{\circ}$ und $75^{\circ}$ und Ionenenergie von $500 \mathrm{eV}$ bis $10 \mathrm{keV}$ beobachtet. Für höhere Energien (30 und $60 \mathrm{keV}$ ) verschwanden die Rippelmuster und nur eine 


\section{Zusammenfassung}

unregelmäßige körnige Struktur wurde beobachtet. RBS-Analysen der bestrahlten Proben zeigen eine signifikante Restkonzentration an implantiertem Edelgas. Der Vergleich mit SDTrimSP-Simulationen zeigt, dass die implantierte Fraktion leicht Werte über $50 \%$ überschreiten kann. Der Verlust von Edelgas wird dann hauptsächlich durch Sputtererosion und Rückreflexion bei größeren Einfallswinkeln bestimmt. Erosion hat hauptsächlich einen stabilisierenden Beitrag bei größeren Einfallswinkeln, und die Musterbildung beruht hauptsächlich auf Massentransport und Ionenimplantation. Die Simulation zeigt auch, dass bei Einfallswinkeln bis etwa $50^{\circ}$ keine Musterbildung auftritt. Die Stabilisierung der Oberfläche in diesem Winkelbereich beruht auf dem positiven Koeffizienten $D_{11}$ und auch dem stabilisierenden Effekt der Edelgasimplantation, d.h. dem Koeffizienten $C_{11}^{\text {implan. }}$.

Im zweiten Teil der Arbeit wurde die ionenstrahlinduzierte Musterbildung auf SiSubstraten mit niederenergetischen $\mathrm{Ne}^{+}$-Ionen untersucht. Bei einer Energie von $500 \mathrm{eV}$ treten ausgeprägte Rippelmuster innerhalb eines Winkelbereichs zwischen etwa $45^{\circ}$ und $60^{\circ}$ auf. Mit der Erhöhung der Ionenenergie auf $1 \mathrm{keV}$ und $1.5 \mathrm{keV}$ verschwindet die Musterbildung vollständig und die Oberfläche bleibt extrem glatt. Das vollständige Verschwinden des Musters, wenn nur die Ionenenergie erhöht wird, liegt an den Krümmungskoeffizienten $C_{11}^{\text {eros. }}$ und $C_{11}^{\text {redist. }}$. Der Erosionskoeffizient $C_{11}^{\text {eros. }}$ ist positiv (stabilisierend) ab einem Einfallswinkel von $45^{\circ}$. Im Gegensatz dazu ist der Redistributionskoeffizient $C_{11}^{\text {redist }}$. negativ (destabilisierend) ab $55^{\circ}$. Das führt dazu, dass der Gesamtkoeffizient $C_{11}^{\text {total }}$ für alle Einfallswinkel positiv bleibt und somit sollte es keine Musterbildung beobachtet werden. Der Implantationskoeffizient $C_{11}^{\text {implant. }}$ ist ein destabilisierender Koeffizient ab einem Ein-

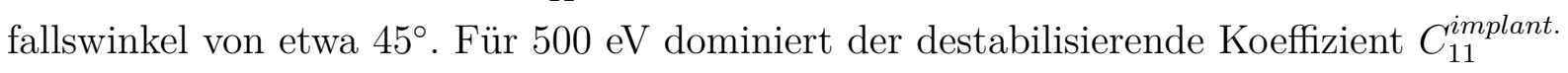
den stabilisierenden Koeffizient $D_{11}$ und führt bei höheren Einfallswinkel zu negativen Gesamtkoeffizient $C_{11}^{\text {total }}$. Für $1 \mathrm{keV}$ und $1.5 \mathrm{keV}$ wird der Sputter-Effekt stärker und der destabilisierende Implantationskoeffizient scheint zu klein zu sein, um den Erosionskoeffizient überwinden zu können. Aus diesem Grund bleibt der Gesamtkoeffizient $C_{11}^{\text {total }}$ positiv im ganzen Winkelbereich. Für krümmungskoeffizienten $C_{22}^{\text {total }}$, die die senkrechten RippelMusterbildung (mit Wellenvektor senkrecht zum projizierten Ionenstrahl) beschreiben, führt Ionenimplantation zu einer signifikanten Stabilisierung der Oberfläche gegen senkrechte Rippelmusterbildung für alle untersuchten Einfallswinkel und Ionenenergien, sodass keine senkrechten Rippelmuster zu erwarten sind.

Im dritten Teil dieser Arbeit wurden Experimente zur Sputter Kodeposition von Ti, Mo, W und Pt während $1 \mathrm{keV} \mathrm{Ar}^{+}$- und $\mathrm{Xe}^{+}$-Ionenerosion von amorphen Kohlenstoffoberflächen präsentiert. Der einfache experimentelle Aufbau mit einem Substrat und 
einem benachbarten geneigten Sputter-Target ermöglichte die Analyse von Mustern für einen breiten Bereich von Surfactant-Abdeckung auf einer einzelnen Probe. Untersuchungen mit Rasterkraftmikroskopie und Transmissionselektronenmikroskopie zeigen, dass die gleichzeitige Kodeposition von Metall-Surfactant-Atomen während der Ionenstrahlerosion zu ausgeprägten Punkt- und Rippelmustern für Ionenfluenzen von $10^{18} / \mathrm{cm}^{2}$ führt. Der Wellenvektor der Rippelmuster zeigt parallel zur projizierten Richtung der einfallenden Surfactant-Atome. Die entstehenden Muster bilden sich unter einem normalen Einfallswinkel, wobei keine Muster für eine saubere Ionenerosion von amorphem Kohlenstoff resultieren, und nur mit gleichzeitiger Kodeposition von Surfactant-Atomen, die stabilen Karbide mit Kohlenstoff bilden. Für Surfactant-Atome, die keine stabilen Karbide mit Kohlenstoff bilden (z.B. Pt), bilden sich keine Muster und die Oberfläche beibt extrem glatt. TEMMessungen von Rippelmustern, die durch Sputter-Kodeposition erzeugt wurden, zeigen eine Bildung von ultradünnen, nanostrukturierten, binären Oberflächenschichten. Abhängig von der chemischen Bindung mit Substrat-Atomen können die Surfactant-Atome zu ultradünnen nanostrukturierten Karbid-Schichten beitragen, die sich auf der amorphen Kohlenstoffschicht bildet.

Die aktuellen Ergebnisse dieser Arbeit können wie folgt zusammengefasst werden:

- Eine Kombination der vorhandenen theoretischen Modelle, einschließlich der vor kurzem eingeführten Erweiterungen kann die ioneninduzierte Musterbildung für einfache Substrate vorhersagen. Insbesondere kann die Abwesenheit von Mustern für die Bestrahlung mit leichten Ionen und bei niedrigen und mittleren Ionenenergien erklärt werden.

- Das Monte-Carlo-Simulationsprogramm SDTrimSP bietet einen ziemlich universellen Ansatz zur Bestimmung von Krümmungskoeffizienten, Sputter-Yield und anderen Eingangsparametern für theoretische Modelle der Musterbildung und ist für fast jede Ionen-Target-Kombination und jede Ionenenergie anwendbar.

- Wir haben die Winkelabhängigkeit der Schichtdicke in unserer Simulation berücksichtigt und finden in vielen Fällen eine gute quantitative Übereinstimmung zwischen theoretischer Vorhersage und Experiment. Die Winkelabhängigkeit der Dicke trägt immer zur Stabilisierung der Oberfläche in der projizierten Richtung des Ionenstrahls bei. Dieser Beitrag scheint für niedrige Ionenenergie und mittlere Einfallswinkel $\left(40^{\circ}-60^{\circ}\right)$ wesentlich zu sein.

- Inkorporation von Edelgasionen in ein Substrat während der Ionenbestrahlung trägt zur Stabilisierung der Oberfläche für Winkel bis zu einem kritischen Wert von etwa 
$45^{\circ}$ bei. Bei größeren Winkeln ist der Beitrag destabilisierend und kann zur Bildung von Rippelmustern beitragen.

- Im Rahmen dieser Arbeit konnten wir durch systematische Untersuchungen der Musterbildung am amorphen Kohlenstoff bei senkrechtem Ioneneinfall mit einer Vielzahl von verschiedenen gleichzeitig kodeponierten Metallatomen einen weiteren eindeutigen Beweis für die ioneninduzierte Phasenseparation als initialen Prozess zur selbstorganisierten Musterbildung unter dem Einfluss von Surfactant-Atomen liefern. 


\section{Ausbilck}

Im ersten und zweiten Teil dieser Arbeit haben wir uns nur mit der Musterbildung auf amorphem Kohlenstoff und Silizium beschäftigt. Eine umfassende Untersuchung der Musterbildung auf Germanium-Oberflächen wurde hier nicht durchgeführt. Jedoch wurden in den letzten experimentellen Studien von ionenstrahlinduzierten Musterbildung auf Ge Rippelmuster nur in einem sehr begrenzten Winkelbereich gefunden und nur für die schwereren Edelgasionen wie $\mathrm{Kr}^{+}$und $\mathrm{Xe}^{+}$. Für die leichteren Ionen wie $\mathrm{Ar}^{+}$und $\mathrm{Ne}^{+}$ verschwand die Musterbildung vollständig [9, 128, 142. Mollick et al. beobachteten anomale und nahezu defekt freie Rippelmuster nach Bestrahlung von Ge-Oberflächen mit $26 \mathrm{keV}$ Au-Ionen bei $60^{\circ}$ [108]. Allerdings lassen sich diesen interessanten Beobachtungen mit den vorhandenen theoretischen Modellen nicht erklären. Es es ist bekannt, dass Germanium dazu neigt, bei Ionenbestrahlung Hohlräume zu bilden. Deswegen ist die Dichte der bestrahlten Schicht nicht mehr konstant und der Einbau von Edelgasionen während der Bestrahlung kann erhöht werden. Darüber hinaus kann eine Dichteänderung ebenfalls den Sputter-Yield beeinflussen. Bis jetzt ist es nicht untersucht, ob diese Effekte einen Einfluss auf die Musterbildung haben. Außerdem existieren noch keine Vergleichsexperimente mit anderen Ionen oder anderen Energien.

In dieser Arbeit haben wir uns nur auf die nullten und ersten Momente der Erosionsund Redistributionskraterfunktionen beschränkt. Allerdings ist es nun möglich mit dem erweiterten Simulationsprogramm SDTrimSP alle Momente der Erosions-und Redistibutionskraterfunktion bis zu vierten Ordnung und bei Bedarf auch Momente höherer Ordnung zu berechnen. Auf diese Weise ist es möglich, alle linearen und nichtlinearen Krümmungskoeffizienten zu berechnen, die zum Beispiel in der Arbeit von Makeev et al. [95] oder R.M. Bradley [96] über die n-ten Ordungsmomente der Kraterfunktionen abgeleitet wurden.

Ein charakteristisches Merkmal des ioneninduzierten Rippel-Musters auf festen Oberflächen ist das dynamische Verhalten, insbesondere die laterale Bewegung der erzeugten Rippelmuster [66]. In der von Bradley und Harper eingeführten linearen Kontinuumstheo- 


\section{Ausbilck}

rie hängt die Geschwindigkeit der Rippel mit der Winkelableitung der Erosionsgeschwindigkeit zusammen und sollte negativ sein ( d.h. entgegen der projizierten Richtung des einfallenden Ionenstrahls) [22]. Die laterale Bewegung von ionenstrahlinduzierten Rippel auf $\mathrm{Si}, \mathrm{SiO}_{2}$, Diamant und Glasoberflächen wurde experimentell unter Verwendung von Rasterelektronenmikroskopen (SEM) in Kombination mit einem fokussierten Ionenstrahl unter Verwendung von Ga-Ionen gemessen [6, 39, 56, 59, 150]. Für $\mathrm{Si}, \mathrm{SiO}_{2}$ und Glasoberflächen bewegen sich die Rippel in die projizierte Richtung des einfallenden GaIonenstrahls. Diese Beobachtungen stehen offensichtlich im Widerspruch zu den Vorhersagen von Bradley und Harper. Mehrere experimentelle Studien haben die Restkonzentration von implantierten Ga-Ionen gemessen und weisen darauf hin, dass Ga-Inkorporation in $\mathrm{Si}$ und $\mathrm{SiO}_{2}$ einen signifikanten Einfluss auf die Rippel-Musterbildung und auch auf die Rippel-Bewegungsgeschwindigkeit haben kann [55, 76, 100. Hofsäss et al. [66] haben eine neuartige Technik zur Messung der Rippel-Bewegung mit hoher Genauigkeit entwickelt. Die Methode basiert auf einer sequenziellen, präzisen Analyse der Verschiebung von Mustern in Bezug auf Markierungsrillen im Mikrometerbereich mit Hilfe fokussierten Ionenstrahlsysteme. Hofsäss et al. konnten die Rippel-Bewegungsgeschwindigkeit für die $\mathrm{Xe}^{+}$-Ionenbestrahlung von reinem Si als Funktion des Ioneneinfallwinkels und für den normalen Einfall von $\mathrm{Xe}^{+}$-Ionen auf Si mit schräger Fe-Kodeposition messen. Jedoch waren die Ergebnisse völlig anders als die bisher einzigen Studien zur Rippel-Bewegung mit Ga-Ionen. Für den ersten Fall finden Hofsäss et al. [66] eine Änderung der RippelBewegungsgeschwindigkeit von negativen zu positiven Geschwindigkeit mit zunehmendem Ioneneinfallswinkel in guter Übereinstimung mit der Vorhersage der BH-Theorie. Für den zweiten Fall finden sie eine Rippel-Bewegung gegen die Richtung der kodeponierten Fe-Surfactant-Atome. Eine Überprüfung der Gültigkeit der theoretischen Musterbildungsmodelle kann realisiert werden, indem man die Rippel-Bewegungsgeschwindigkeit an amorphen $\mathrm{C}$, $\mathrm{Si}$ und $\mathrm{SiO}_{2}$ nach Bestrahlung mit Edelgasionen untersucht und sie mit der Rippel-Bewegungsgeschwindigkeit, die nach Ga- oder Metall-Ionenbestrahlung des gleichen Substrats beobachtet wird, vergleicht.

In mehreren experimentellen Studien wurde eine ungewöhnliche Bildung von Punktmustern für einen normalen und nahezu normalen Ioneneinfall auf Verbindungshalbleitern wie GaSb, InSb, InP und GaAs beobachtet [48 50, 92, 125]. Diese Musterbildung wurde als selbsterhaltende Ätzmaskierung infolge fortgesetzter Trennung einer Komponente als Ergebnis des bevorzugten Sputterns erklärt [88]. Dieser Mechanismus ist dem Surfactant-Sputtern ziemlich ähnlich. Jedoch werden in diesem Fall die Surfactant-Atome nicht deponiert, sondern durch bevorzugtes Sputtern an der Substratoberfläche kontinu- 
ierlich angereichert. Ein ähnlicher Ansatz zur Punktmusterbildung, der bisher nicht untersucht wurde, wäre die Verwendung von Dünnschichten aus amorphem Si-Metall oder C-Metallverbindungen als Substrate mit variabler Metallkonzentration. 



\section{Literaturverzeichnis}

[1] Colutron research corporation. http://colutron.com/.

[2] FACT, Facility for the analysis of chemical thermodynamics, Centre de Recherche en Calcul Thermochimique, Ecole Polytechnique, Universite de Montreal. http: //crct.polymtl.ca/fact/.

[3] Fraunhofer Institut für Werkstoff- und Strahltechnik. http://iws.fraunhofer. $\mathrm{de} /$.

[4] Institut für Angewandte Physik, Karlsruher Institut für Technologie (KIT). http: //aph-ags.webarchiv.kit.edu/

[5] Tectra GmbH. http://tectra.de/.

[6] PFA Alkemade. Propulsion of ripples on glass by ion bombardment. Physical review letters, 96(10):107602, 2006.

[7] II Amirov, IV Zhuravlev, DS Kibalov, PA Lepshin, and VK Smirnov. Plasmachemical etching of wave-ordered structure formed on amorphous silicon surface by nitrogen ion bombardment. Physics of low-dimensional structures, 9:51-58, 2003.

[8] Eitan Anzenberg, Charbel S Madi, Michael J Aziz, and Karl F Ludwig Jr. Timeresolved measurements of nanoscale surface pattern formation kinetics in two dimensions on ion-irradiated Si. Physical Review B, 84(21):214108, 2011.

[9] Eitan Anzenberg, Joy C Perkinson, Charbel S Madi, Michael J Aziz, and Karl F Ludwig Jr. Nanoscale surface pattern formation kinetics on germanium irradiated by $\mathrm{Kr}^{+}$ions. Physical Review B, 86(24):245412, 2012.

[10] Tomaso Aste and U Valbusa. Ripples and ripples: from sandy deserts to ionsputtered surfaces. New Journal of Physics, 7(1):122, 2005.

[11] VI Bachurin, PA Lepshin, VK Smirnov, and AB Churilov. Infrared spectroscopy of a silicon surface bombarded by nitrogen ions. Technical Physics Letters, 24(3):214216, 1998. 
[12] Matthew J Banholzer, Jill E Millstone, Lidong Qin, and Chad A Mirkin. Rationally designed nanostructures for surface-enhanced raman spectroscopy. Chemical Society Reviews, 37(5):885-897, 2008.

[13] Tanmoy Basu, Jyoti Ranjan Mohanty, and T Som. Unusual pattern formation on Si (100) due to low energy ion bombardment. Applied Surface Science, 258(24):99449948, 2012.

[14] S Bhattacharjee, P Karmakar, and A Chakrabarti. Key factors of ion induced nanopatterning. Nuclear Instruments and Methods in Physics Research Section B: Beam Interactions with Materials and Atoms, 278:58-62, 2012.

[15] S Bhattacharjee, P Karmakar, V Naik, AK Sinha, and A Chakrabarti. The role of carbon in ion beam nano-patterning of silicon. Applied Physics Letters, 103(18):181601, 2013.

[16] F Bisio, R Moroni, F Buatier de Mongeot, M Canepa, and L Mattera. Tuning the magnetic anisotropy of ultrathin $\mathrm{Fe} / \mathrm{Ag}$ (001) films from biaxial to uniaxial by ion sculpting. Applied physics letters, 89(5):052507, 2006.

[17] Omar Bobes. Die Rolle des Massentranportes und des krümmungsabhängigen Sputterprozesses bei der ionenstrahlinduzierten Musterbildung, Diplomarbeit, Universität Göttingen (2013).

[18] Omar Bobes, Hans Hofsäss, and Kun Zhang. Neon ion beam induced pattern formation on amorphous carbon surfaces. AIP Advances, 8(2):025205, 2018.

[19] Omar Bobes, Kun Zhang, and Hans Hofsäss. Ion beam induced surface patterns due to mass redistribution and curvature-dependent sputtering. Physical Review B, 86(23):235414, 2012.

[20] R Mark Bradley. Exact linear dispersion relation for the sigmund model of ion sputtering. Physical Review B, 84(7):075413, 2011.

[21] R Mark Bradley. Producing ripple topographies by ion bombardment with codeposition of impurities: A curvature-dependent sputter yield is not required. Physical Review B, 85(11):115419, 2012.

[22] R Mark Bradley and James ME Harper. Theory of ripple topography induced by ion bombardment. Journal of Vacuum Science \&5 Technology A: Vacuum, Surfaces, and Films, 6(4):2390-2395, 1988. 
[23] R Mark Bradley and Hans Hofsäss. Nanoscale patterns produced by self-sputtering of solid surfaces: The effect of ion implantation. Journal of Applied Physics, 120(7):074302, 2016.

[24] Marc Brötzmann. Wachstum und Charakterisierung von BN/ZnO- sowie BN/SiHeterostruktured, Diplomarbeit, Universität Göttingen (2008).

[25] Felix Büttner. Schichtdickenabhängigkeit der magnetischen Eingenschaft von strukturierten einkristallinen Eisenfilmen, Diplomarbeit; Universität Göttingen (2010).

[26] Felix Büttner, Kun Zhang, Susanne Seyffarth, Tobias Liese, Hans-Ulrich Krebs, CAF Vaz, and Hans Hofsäss. Thickness dependence of the magnetic properties of ripple-patterned Fe/Mgo (001) films. Physical Review B, 84(6):064427, 2011.

[27] G Carter. Viscoelastic buckling and plastic flowâdeterministic mechanisms for ripple initiation on ion-bombarded amorphous solids. Surface and Interface Analysis, 25(12):952-955, 1997.

[28] G Carter and V Vishnyakov. Roughening and ripple instabilities on ion-bombarded Si. Physical Review B, 54(24):17647, 1996.

[29] G Carter, V Vishnyakov, Yu V Martynenko, and MJ Nobes. The effects of ion species and target temperature on topography development on ion bombarded Si. Journal of applied physics, 78(6):3559-3565, 1995.

[30] G Carter, V Vishnyakov, and MJ Nobes. Ripple topography development on ion bombarded Si. Nuclear Instruments and Methods in Physics Research Section B: Beam Interactions with Materials and Atoms, 115(1-4):440-445, 1996.

[31] Mario Castro and Rodolfo Cuerno. Ion induced solid flow. arXiv preprint arXiv:1007.2144, 2010.

[32] Mario Castro and Rodolfo Cuerno. Hydrodynamic approach to surface pattern formation by ion beams. Applied Surface Science, 258(9):4171-4178, 2012.

[33] Mario Castro, R Gago, Luis Vázquez, Javier Muñoz-García, and Rodolfo Cuerno. Stress-induced solid flow drives surface nanopatterning of silicon by ion-beam irradiation. Physical Review B, 86(21):214107, 2012.

[34] Wai Lun Chan and Eric Chason. Making waves: kinetic processes controlling surface evolution during low energy ion sputtering. Journal of Applied Physics, 101(12):1, 2007. 
[35] YJ Chen, JP Wang, EW Soo, L Wu, and TC Chong. Periodic magnetic nanostructures on self-assembled surfaces by ion beam bombardment. Journal of applied physics, 91(10):7323-7325, 2002.

[36] TK Chini, F Okuyama, Masaki Tanemura, and K Nordlund. Structural investigation of keV Ar-ion-induced surface ripples in Si by cross-sectional transmission electron microscopy. Physical Review B, 67(20):205403, 2003.

[37] Rodolfo Cuerno and Albert-László Barabási. Dynamic scaling of ion-sputtered surfaces. Physical review letters, 74(23):4746, 1995.

[38] Rodolfo Cuerno, Hernán A Makse, Silvina Tomassone, Stephen T Harrington, and H Eugene Stanley. Stochastic model for surface erosion via ion sputtering: Dynamical evolution from ripple morphology to rough morphology. Physical Review Letters, 75(24):4464, 1995.

[39] A Datta, Yuh-Renn Wu, and YL Wang. Real-time observation of ripple structure formation on a diamond surface under focused ion-beam bombardment. Physical Review B, 63(12):125407, 2001.

[40] Benny Davidovitch, Michael J Aziz, and Michael P Brenner. On the stabilization of ion sputtered surfaces. Physical Review B, 76(20):205420, 2007.

[41] Benny Davidovitch, Michael J Aziz, and Michael P Brenner. Linear dynamics of ion sputtered surfaces: instability, stability and bifurcations. Journal of Physics: Condensed Matter, 21(22):224019, 2009.

[42] W Eckstein, R Dohmen, A Mutzke, and R Schneider. SDTrimSP: A Monte-Carlo code for calculating collision phenomena in randomized targets. IPP report, 12(3):40, 2007.

[43] Wolfgang Eckstein and Herbert Urbassek. Computer simulation of the sputtering process. Sputtering by Particle Bombardment, pages 21-31, 2007.

[44] Osman El-Atwani, Sean Gonderman, Alexander DeMasi, Anastassiya Suslova, Justin Fowler, Mohamad El-Atwani, Karl Ludwig, and Jean Paul Allain. Nanopatterning of metal-coated silicon surfaces via ion beam irradiation: Real time x-ray studies reveal the effect of silicide bonding. Journal of Applied Physics, 113(12):124305, 2013. 
[45] K Elst, Wilfried Vandervorst, J Alay, Johan Snauwaert, and Louis Hellemans. Influence of oxygen on the formation of ripples on Si. Journal of Vacuum Science $\&$ Technology B: Microelectronics and Nanometer Structures Processing, Measurement, and Phenomena, 11(6):1968-1981, 1993.

[46] Jonah Erlebacher, Michael J Aziz, Eric Chason, Michael B Sinclair, and Jerrold A Floro. Spontaneous pattern formation on ion bombarded Si (001). Physical review letters, 82(11):2330, 1999.

[47] Jonah Erlebacher, Michael J Aziz, Eric Chason, Michael B Sinclair, and Jerrold A Floro. Nonlinear amplitude evolution during spontaneous patterning of ion-bombarded Si (001). Journal of Vacuum Science 85 Technology A: Vacuum, Surfaces, and Films, 18(1):115-120, 2000.

[48] S Facsko, T Bobek, T Dekorsy, and H Kurz. Ordered quantum dot formation by ion sputtering. physica status solidi (b), 224(2):537-540, 2001.

[49] Stefan Facsko, Thomas Bobek, Heinrich Kurz, Thomas Dekorsy, Stepan Kyrsta, and $\mathrm{R}$ Cremer. Ion-induced formation of regular nanostructures on amorphous GaSb surfaces. Applied Physics Letters, 80(1):130-132, 2002.

[50] Stefan Facsko, Thomas Dekorsy, Clemens Koerdt, Cyril Trappe, Heinrich Kurz, Alexander Vogt, and Hans L Hartnagel. Formation of ordered nanoscale semiconductor dots by ion sputtering. Science, 285(5433):1551-1553, 1999.

[51] Barbara Fazio, Cristiano DâAndrea, Francesco Bonaccorso, Alessia Irrera, Giuseppe Calogero, Cirino Vasi, Pietro Giuseppe Gucciardi, Maria Allegrini, Andrea Toma, Daniele Chiappe, et al. Re-radiation enhancement in polarized surface-enhanced resonant Raman scattering of randomly oriented molecules on self-organized gold nanowires. Acs Nano, 5(7):5945-5956, 2011.

[52] François Flory, Ludovic Escoubas, and Gérard Berginc. Optical properties of nanostructured materials: a review. Journal of Nanophotonics, 5(1):052502, 2011.

[53] A. Weidinger G. Schatz. Nukleare Festkörperphysik, Teubner-Verlag, 1997.

[54] PK Giri, V Raineri, G Franzo, and E Rimini. Mechanism of swelling in low-energy ion-irradiated silicon. Physical Review B, 65(1):012110, 2001.

[55] Hubert Gnaser, Alexander Brodyanski, and Bernhard Reuscher. Focused ion beam implantation of $\mathrm{Ga}$ in $\mathrm{Si}$ and Ge: fluence-dependent retention and surface morphology. Surface and Interface Analysis, 40(11):1415-1422, 2008. 
[56] Hubert Gnaser, Bernhard Reuscher, and Anna Zeuner. Propagation of nanoscale ripples on ion-irradiated surfaces. Nuclear Instruments and Methods in Physics Research Section B: Beam Interactions with Materials and Atoms, 285:142-147, 2012.

[57] C. Ronning H. Hofsäss. Ionenstrahlpysik und Nukleare Festkörperphysik, Jahresbericht, II. physikalisches Institut, Universität Göttingen (2000).

[58] S Habenicht. Morphology of graphite surfaces after ion-beam erosion. Physical Review B, 63(12):125419, 2001.

[59] S Habenicht, KP Lieb, J Koch, and AD Wieck. Ripple propagation and velocity dispersion on ion-beam-eroded silicon surfaces. Physical Review B, 65(11):115327, 2002.

[60] Sönke Habenicht, Wolfgang Bolse, and Klaus-Peter Lieb. A low-energy ion implanter for surface and materials science. Review of scientific instruments, 69(5):2120-2126, 1998.

[61] Matt P Harrison and R Mark Bradley. Crater function approach to ion-induced nanoscale pattern formation: Craters for flat surfaces are insufficient. Physical Review $B, 89(24): 245401,2014$.

[62] Alexander K Hartmann, Reiner Kree, and Taha Yasseri. Simulating discrete models of pattern formation by ion beam sputtering. Journal of Physics: Condensed Matter, $21(22): 224015,2009$.

[63] Conyers Herring. Effect of change of scale on sintering phenomena. Journal of Applied Physics, 21(4):301-303, 1950.

[64] H Hofsäss, H Binder, T Klumpp, and E Recknagel. Doping and growth of diamondlike carbon films by ion beam deposition. Diamond and Related Materials, 3(12):137-142, 1994.

[65] H Hofsäss, O Bobes, and K Zhang. Argon ion beam induced surface pattern formation on Si. Journal of Applied Physics, 119(3):035302, 2016.

[66] H Hofsäss, K Zhang, HG Gehrke, and C Brüsewitz. Propagation of ripple patterns on Si during ion bombardment. Physical Review B, 88(7):075426, 2013.

[67] H Hofsäss, K Zhang, and A Mutzke. Simulation of ion beam sputtering with SDTrimsp, TRIDYN and SRIM. Applied Surface Science, 310:134-141, 2014. 
[68] H Hofsäss, K Zhang, A Pape, O Bobes, and M Brötzmann. The role of phase separation for self-organized surface pattern formation by ion beam erosion and metal atom co-deposition. Applied Physics A, 111(2):653-664, 2013.

[69] H Hofsäss, K Zhang, and H Zutz. Nanostructured carbide surfaces prepared by surfactant sputtering. Nuclear Instruments and Methods in Physics Research Section B: Beam Interactions with Materials and Atoms, 267(8):1398-1402, 2009.

[70] Hans Hofsäss. Surface instability and pattern formation by ion-induced erosion and mass redistribution. Applied Physics A, 114(2):401-422, 2014.

[71] Hans Hofsäss. Model for roughening and ripple instability due to ion-induced mass redistribution [addendum to H. Hofsäss, Appl. Phys. A 114 (2014) 401,âSurface instability and pattern formation by ion-induced erosion and mass redistributionâ]. Applied Physics A, 119(2):687-695, 2015.

[72] Hans Hofsäss. Quantitative Description of Ion Beam Induced Surface Pattern Formation, Project Description, Universität Göttingen (2014).

[73] Hans Hofsäss and R Mark Bradley. Tests of the modified sigmund model of ion sputtering using monte carlo simulations. Journal of Applied Physics, 117(17):174310, 2015 .

[74] Hans Hofsäss and Kun Zhang. Surfactant sputtering. Applied Physics A: Materials Science \& Processing, 92(3):517-524, 2008.

[75] Hans Hofsäss, Kun Zhang, and Omar Bobes. Self-organized surface ripple pattern formation by ion implantation. Journal of Applied Physics, 120(13):135308, 2016.

[76] DE Hole, PD Townsend, JD Barton, LC Nistor, and J Van Landuyt. Gallium colloid formation during ion implantation of glass. Journal of non-crystalline solids, 180(23):266-274, 1995.

[77] Y Homma, A Takano, and Y Higashi. Oxygen-ion-induced ripple formation on silicon: evidence for phase separation and tentative model. Applied surface science, 203:35-38, 2003.

[78] MZ Hossain, K Das, JB Freund, and HT Johnson. Ion impact crater asymmetry determines surface ripple orientation. Applied Physics Letters, 99(15):151913, 2011.

[79] MZ Hossain, JB Freund, and HT Johnson. Ion impact energy distribution and sputtering of Si and Ge. Journal of Applied Physics, 111(10):103513, 2012. 
[80] Qin $\mathrm{Hu}$ and William O’Neill. Focused-Ion-Beam Induced Nano Feature SelfAssembly on Glassy Carbon. Journal of nanoscience and nanotechnology, 11(6):5394-5401, 2011.

[81] N Kalyanasundaram, JB Freund, and HT Johnson. A multiscale crater function model for ion-induced pattern formation in silicon. Journal of Physics: Condensed Matter, 21(22):224018, 2009.

[82] N Kalyanasundaram, M Ghazisaeidi, JB Freund, and HT Johnson. Single impact crater functions for ion bombardment of silicon. Applied Physics Letters, 92(13):131909, 2008.

[83] Adrian Keller and Stefan Facsko. Tuning the quality of nanoscale ripple patterns by sequential ion-beam sputtering. Physical Review B, 82(15):155444, 2010.

[84] Hyunhyub Ko, Srikanth Singamaneni, and Vladimir V Tsukruk. Nanostructured surfaces and assemblies as SERS media. Small, 4(10):1576-1599, 2008.

[85] R Kree, T Yasseri, and AK Hartmann. Surfactant Sputtering: Theory of a new method of surface nanostructuring by ion beams. Nuclear Instruments and Methods in Physics Research Section B: Beam Interactions with Materials and Atoms, 267(8):1403-1406, 2009.

[86] Rammohan Kriparamanan, Pranesh Aswath, Anhong Zhou, Liping Tang, and Kytai T Nguyen. Nanotopography: cellular responses to nanostructured materials. Journal of nanoscience and nanotechnology, 6(7):1905-1919, 2006.

[87] S. M. Langkat. Tieftemperatur-Rasterkraftmikroskop auf antiferromagnetischen übergangsmatalloxiden, Doktorarbeit; Universität Hamburg (2002).

[88] Sébastien Le Roy, Etienne Barthel, Nathalie Brun, Anne Lelarge, and E Søndergård. Self-sustained etch masking: A general concept to initiate the formation of nanopatterns during ion erosion. Journal of Applied Physics, 106(9):094308, 2009.

[89] MO Liedke, B Liedke, A Keller, B Hillebrands, A Mücklich, S Facsko, and J Fassbender. Induced anisotropies in exchange-coupled systems on rippled substrates. Physical Review B, 75(22):220407, 2007.

[90] ZX Liu and PFA Alkemade. Flux dependence of oxygen-beam-induced ripple growth on silicon. Applied Physics Letters, 79(26):4334-4336, 2001. 
[91] Sven Macko, Frank Frost, Martin Engler, Dietmar Hirsch, Thomas Höche, Jörg Grenzer, and Thomas Michely. Phenomenology of iron-assisted ion beam pattern formation on Si (001). New Journal of Physics, 13(7):073017, 2011.

[92] SW MacLaren, JE Baker, NL Finnegan, and CM Loxton. Surface roughness development during sputtering of GaAs and InP: Evidence for the role of surface diffusion in ripple formation and sputter cone development. Journal of Vacuum Science \& Technology A: Vacuum, Surfaces, and Films, 10(3):468-476, 1992.

[93] Charbel S Madi, Eitan Anzenberg, Karl F Ludwig Jr, and Michael J Aziz. Mass redistribution causes the structural richness of ion-irradiated surfaces. Physical review letters, 106(6):066101, 2011.

[94] Charbel S Madi, H Bola George, and Michael J Aziz. Linear stability and instability patterns in ion-sputtered silicon. Journal of Physics: Condensed Matter, 21(22):224010, 2009.

[95] Maxim A Makeev, Rodolfo Cuerno, and Albert-Laszlo Barabasi. Morphology of ionsputtered surfaces. Nuclear Instruments and Methods in Physics Research Section B: Beam Interactions with Materials and Atoms, 197(3-4):185-227, 2002.

[96] R Mark Bradley. Exact linear-dispersion relation for the sigmund model of ion sputtering (5 pages) 075413. Physical Review-Section B-Condensed Matter, 84(7), 2011.

[97] R Mark Bradley and Hans Hofsäss. A modification to the sigmund model of ion sputtering. Journal of Applied Physics, 116(23):234304, 2014.

[98] Thomas Michael Mayer, David Price Adams, MJ Vasile, and KM Archuleta. Morphology evolution on diamond surfaces during ion sputtering. Journal of Vacuum Science 6 Technology A: Vacuum, Surfaces, and Films, 23(6):1579-1587, 2005.

[99] SG Mayr, Y Ashkenazy, K Albe, and RS Averback. Mechanisms of radiation-induced viscous flow: Role of point defects. Physical review letters, 90(5):055505, 2003.

[100] R Menzel, K Gärtner, W Wesch, and H Hobert. Damage production in semiconductor materials by a focused $\mathrm{Ga}^{+}$ion beam. Journal of Applied Physics, 88(10):5658$5661,2000$.

[101] P Mishra and D Ghose. Formation of nanoripples in $\mathrm{Al}$ films during $\mathrm{O}_{2}^{+}$sputtering. Physical Review B, 74(15):155427, 2006. 
Literaturverzeichnis

[102] P Mishra and D Ghose. The rotation of ripple pattern and the shape of the collision cascade in ion sputtered thin metal films. Journal of Applied Physics, 104(9):094305, 2008.

[103] P Mishra and D Ghose. Effect of initial target surface roughness on the evolution of ripple topography induced by oxygen sputtering of Al films. Journal of Applied Physics, 105(1):014304, 2009.

[104] P Mishra, P Karmakar, and D Ghose. Electrical characterization of oxygen-induced nanosized ripples on aluminum thin films by conductive atomic force microscopy. Nuclear Instruments and Methods in Physics Research Section B: Beam Interactions with Materials and Atoms, 243(1):16-19, 2006.

[105] W Möller and W Eckstein. TridynâA TRIM simulation code including dynamic composition changes. Nuclear Instruments and Methods in Physics Research Section B: Beam Interactions with Materials and Atoms, 2(1-3):814-818, 1984.

[106] Wolfhard Möller, Wolfgang Eckstein, and JP Biersack. Tridyn-binary collision simulation of atomic collisions and dynamic composition changes in solids. Computer Physics Communications, 51(3):355-368, 1988.

[107] SA Mollick and D Ghose. Formation and characterization of perpendicular mode Si ripples by glancing angle $\mathrm{O}_{2}^{+}$sputtering at room temperature. Journal of Applied Physics, 106(4):044309, 2009.

[108] Safiul Alam Mollick, Debabrata Ghose, Patrick D Shipman, and R Mark Bradley. Anomalous patterns and nearly defect-free ripples produced by bombarding silicon and germanium with a beam of gold ions. Applied Physics Letters, 104(4):043103, 2014.

[109] Michael Moseler, Peter Gumbsch, Cinzia Casiraghi, Andrea C Ferrari, and John Robertson. The ultrasmoothness of diamond-like carbon surfaces. Science, 309(5740):1545-1548, 2005.

[110] William W Mullins. Flattening of a nearly plane solid surface due to capillarity. Journal of Applied Physics, 30(1):77-83, 1959.

[111] Javier Muñoz-García, Rodolfo Cuerno, and Mario Castro. Coupling of morphology to surface transport in ion-beam irradiated surfaces: Oblique incidence. Physical Review B, 78(20):205408, 2008. 
[112] Javier Muñoz-García, Raúl Gago, Luis Vázquez, José Angel Sánchez-García, and Rodolfo Cuerno. Observation and modeling of interrupted pattern coarsening: surface nanostructuring by ion erosion. Physical review letters, 104(2):026101, 2010.

[113] Andreas Mutzke, Ralf Schneider, Wolfgang Eckstein, and Renate Dohmen. SDTrimSP Version 5.00. IPP report, 12(8), 2011.

[114] M Navez, C Sella, and D Chaperot. Investigation of the attack on glass by ion bombardment. CR Acad. Sci. Paris, 254:240-244, 1962.

[115] M Navez, C Sella, and D Chaperot. Nonlinear ripple dynamics on amorphous surfaces patterned by ion beam sputtering. Czetch Repub. Acad. Sci, 254:240, 1962.

[116] Maureen L Nietiadi and Herbert M Urbassek. Influence of local curvature on sputtering. Applied Physics Letters, 103(11):113108, 2013.

[117] Scott A Norris. Stability analysis of a viscoelastic model for ion-irradiated silicon. Physical Review B, 85(15):155325, 2012.

[118] Scott A Norris, Michael P Brenner, and Michael J Aziz. From crater functions to partial differential equations: a new approach to ion bombardment induced nonequilibrium pattern formation. Journal of Physics: Condensed Matter, 21(22):224017, 2009.

[119] Scott A Norris, Juha Samela, Laura Bukonte, Marie Backman, Flyura Djurabekova, Kai Nordlund, Charbel S Madi, Michael P Brenner, and Michael J Aziz. Molecular dynamics of single-particle impacts predicts phase diagrams for large scale pattern formation. Nature communications, 2:276, 2011.

[120] Scott A Norris, Juha Samela, Matias Vestberg, Kai Nordlund, and Michael J Aziz. Crater functions for compound materials: A route to parameter estimation in coupled-pde models of ion bombardment. Nuclear Instruments and Methods in Physics Research Section B: Beam Interactions with Materials and Atoms, 318:245-252, 2014.

[121] Thomas WH Oates, Adrian Keller, Stefan Facsko, and Arndt Mücklich. Aligned silver nanoparticles on rippled silicon templates exhibiting anisotropic plasmon absorption. Plasmonics, 2(2):47-50, 2007.

[122] S. E. Orchard. On surface levelling in viscous liquids. Appl. sci. Res. A, 11:451, 1962. 
Literaturverzeichnis

[123] Alexander Oron, Stephen H Davis, and S George Bankoff. Long-scale evolution of thin liquid films. Reviews of modern physics, 69(3):931, 1997.

[124] Shahjada A Pahlovy, SF Mahmud, K Yanagimoto, and I Miyamoto. Ripple formation on atomically flat cleaved Si surface with roughness of $0.038 \mathrm{~nm} \mathrm{rms}$ by low-energy $\mathrm{ar}^{+}$ion bombardment. Journal of Vacuum Science $\&$ Technology A: Vacuum, Surfaces, and Films, 29(2):021015, 2011.

[125] Dipak Paramanik, SN Sahu, and Shikha Varma. Morphological evolution of InP nano-dots and surface modifications after keV irradiation. Journal of Physics D: Applied Physics, 41(12):125308, 2008.

[126] S Park, B Kahng, H Jeong, and A-L Barabási. Dynamics of ripple formation in sputter erosion: nonlinear phenomena. Physical review letters, 83(17):3486, 1999.

[127] Joy C Perkinson, Eitan Anzenberg, Michael J Aziz, and Karl F Ludwig Jr. Modelindependent test of the truncated crater function theory of surface morphology evolution during ion bombardment. Physical Review B, 89(11):115433, 2014.

[128] Joy C Perkinson, Charbel S Madi, and Michael J Aziz. Nanoscale topographic pattern formation on $\mathrm{Kr}^{+}$-bombarded germanium surfaces. Journal of Vacuum Science \& Technology A: Vacuum, Surfaces, and Films, 31(2):021405, 2013.

[129] HX Qian and W Zhou. Ripple rotation on ion sputtered Si (100). Materials Letters, 77:113-116, 2012.

[130] C Ronning and H Hofsäss. Proceeding of the Fourth International Symposium on Diamond Materials, Reno, Nevada, 1995. The Electrochemical Society, 1995.

[131] AS Rudy and VK Smirnov. Hydrodynamic model of wave-ordered structures formed by ion bombardment of solids. Nuclear Instruments and Methods in Physics Research Section B: Beam Interactions with Materials and Atoms, 159(1-2):52-59, 1999.

[132] S Sarkar, B Van Daele, and Wilfried Vandervorst. Ripple morphologies on ion irradiated Si 1- x Ge x. Applied Surface Science, 255(4):1368-1372, 2008.

[133] Kenji Sato, Iwao Okamoto, Yoshito Kitamoto, and Shoji Ishida. Oblique ion nanotexturing technology for longitudinal recording media. Japanese Journal of Applied Physics, 46(8R):5139, 2007. 
[134] VB Shenoy, WL Chan, and E Chason. Compositionally modulated ripples induced by sputtering of alloy surfaces. Physical review letters, 98(25):256101, 2007.

[135] G Sidenius. The high temperature hollow cathode ion source. Nuclear Instruments and Methods, 38:19-22, 1965.

[136] Peter Sigmund. Theory of sputtering. i. sputtering yield of amorphous and polycrystalline targets. Physical review, 184(2):383, 1969.

[137] Peter Sigmund. A mechanism of surface micro-roughening by ion bombardment. Journal of Materials Science, 8(11):1545-1553, 1973.

[138] Ajay Vikram Singh, Varun Vyas, Rajendra Patil, Vimal Sharma, Pasquale Emanuele Scopelliti, Gero Bongiorno, Alessandro Podesta, Cristina Lenardi, Wasudev Namdev Gade, and Paolo Milani. Quantitative characterization of the influence of the nanoscale morphology of nanostructured surfaces on bacterial adhesion and biofilm formation. PloS one, 6(9):e25029, 2011.

[139] VK Smirnov, DS Kibalov, SA Krivelevich, PA Lepshin, EV Potapov, RA Yankov, W Skorupa, VV Makarov, and AB Danilin. Wave-ordered structures formed on SOI wafers by reactive ion beams. Nuclear Instruments and Methods in Physics Research Section B: Beam Interactions with Materials and Atoms, 147(1):310-315, 1999.

[140] VK Smirnov, DS Kibalov, OM Orlov, and VV Graboshnikov. Technology for nanoperiodic doping of a metal-oxide-semiconductor field-effect transistor channel using a self-forming wave-ordered structure. Nanotechnology, 14(7):709, 2003.

[141] E Snoeks, T Weber, A Cacciato, and A Polman. Mev ion irradiation-induced creation and relaxation of mechanical stress in silica. Journal of applied physics, 78(7):4723-4732, 1995.

[142] Marc Teichmann, Jan Lorbeer, Bashkim Ziberi, Frank Frost, and Bernd Rauschenbach. Pattern formation on Ge by low energy ion beam erosion. New Journal of Physics, 15(10):103029, 2013.

[143] A Toma, D Chiappe, C Boragno, and F Buatier De Mongeot. Self-organized ionbeam synthesis of nanowires with broadband plasmonic functionality. Physical Review B, 81(16):165436, 2010. 
[144] Christopher C Umbach, Randall L Headrick, and Kee-Chul Chang. Spontaneous nanoscale corrugation of ion-eroded $\mathrm{SiO}_{2}$ : the role of ion-irradiation-enhanced viscous flow. Physical review letters, 87(24):246104, 2001.

[145] Herbert M Urbassek. Molecular-dynamics simulation of sputtering. Nuclear Instruments and Methods in Physics Research Section B: Beam Interactions with Materials and Atoms, 122(3):427-441, 1997.

[146] John J Vajo, Robert E Doty, and Eun-Hee Cirlin. Influence of $\mathrm{O}_{2}^{+}$energy, flux, and fluence on the formation and growth of sputtering-induced ripple topography on silicon. Journal of Vacuum Science \& Technology A: Vacuum, Surfaces, and Films, 14(5):2709-2720, 1996.

[147] U Valbusa, C Boragno, and F Buatier De Mongeot. Nanostructuring surfaces by ion sputtering. Journal of Physics: Condensed Matter, 14(35):8153, 2002.

[148] Sebastian Vauth and SG Mayr. Relevance of surface viscous flow, surface diffusion, and ballistic effects in kev ion smoothing of amorphous surfaces. Physical Review $B, 75(22): 224107,2007$.

[149] V Vishnyakov and G Carter. $\mathrm{Ne}^{+}, \mathrm{Ar}^{+}$and $\mathrm{Xe}^{+}$ion bombardment induced and suppressed topography on Si. Nuclear Instruments and Methods in Physics Research Section B: Beam Interactions with Materials and Atoms, 106(1-4):174-178, 1995.

[150] Qiangmin Wei, Jie Lian, Lynn A Boatner, LM Wang, and Rodney C Ewing. Propagation of ripples on pyrochlore induced by ion beam bombardment. Physical Review $B, 80(8): 085413,2009$.

[151] CJWMM-AN Wei-Kan, JW Mayer, and MA Nicolet. Backscatering Spectroscopy, 1978.

[152] Qing-feng Zhan, Stijn Vandezande, Chris Van Haesendonck, and Kristiaan Temst. Manipulation of in-plane uniaxial anisotropy in $\mathrm{Fe} / \mathrm{MgO}$ (001) films by ion sputtering. Applied Physics Letters, 91(12):122510, 2007.

[153] K Zhang, O Bobes, and H Hofsäss. Designing self-organized nanopatterns on Si by ion irradiation and metal co-deposition. Nanotechnology, 25(8):085301, 2014.

[154] K Zhang, M Brötzmann, and H Hofsäss. Sharp transition from ripple patterns to a flat surface for ion beam erosion of si with simultaneous co-deposition of iron. AIP Advances, 2(3):032123, 2012. 
[155] K Zhang, F Rotter, M Uhrmacher, C Ronning, H Hofsäss, and J Krauser. Pattern formation of Si surfaces by low-energy sputter erosion. Surface and Coatings Technology, 201(19):8299-8302, 2007.

[156] K Zhang, M Uhrmacher, H Hofsäss, and J Krauser. Magnetic texturing of ferromagnetic thin films by sputtering induced ripple formation. Journal of Applied Physics, 103(8):083507, 2008.

[157] Kun Zhang, Marc Brötzmann, and Hans Hofsäss. Surfactant-driven self-organized surface patterns by ion beam erosion. New Journal of Physics, 13(1):013033, 2011.

[158] XD Zhu, H Naramoto, Y Xu, K Narumi, and K Miyashita. Tuning surface morphologies of ion-assisted diamondlike carbon film on the nanometer scale. The Journal of chemical physics, 116(23):10458-10461, 2002.

[159] B Ziberi, F Frost, Th Höche, and B Rauschenbach. Ripple pattern formation on silicon surfaces by low-energy ion-beam erosion: Experiment and theory. Physical Review B, 72(23):235310, 2005.

[160] B Ziberi, F Frost, B Rauschenbach, and Th Höche. Highly ordered self-organized dot patterns on Si surfaces by low-energy ion-beam erosion. Applied Physics Letters, 87(3):033113, 2005.

[161] James F Ziegler, Matthias D Ziegler, and Jochen P Biersack. SRIM: the stopping and range of ions in matter. Cadence Design Systems, 2008.

[162] James F Ziegler, Matthias D Ziegler, and Jochen P Biersack. SRIM-The stopping and range of ions in matter (2010). Nuclear Instruments and Methods in Physics Research Section B: Beam Interactions with Materials and Atoms, 268(11):18181823, 2010.

[163] Hayo Zutz. Wachstum von Kohlenstoff-Metall-Nanokompitschichten, Diplomarbeit, Universität Göttingen (2005). 



\section{Abbildungsverzeichnis}

2.1. Die gaußförmige Energieverteilung des einfallenden Ions. Die Isolinien ver-

\begin{tabular}{|l|l|}
\hline binden Punkte gleicher Energiedeposition. Das Maximum der Funktion \\
\hline \hline$F_{D}(E, \theta)$ liegt in der Mitte (aus [137]). . . . . . . . . . . . . . . . 6
\end{tabular}

2.2. Krümmungsabhängige Energieverteilung von Ionen a) bei senkrechtem Ein-

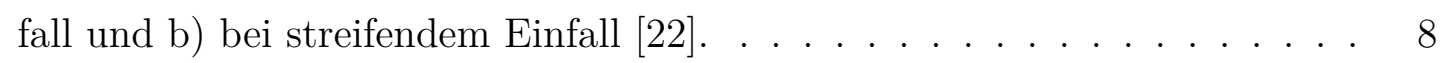

2.3. Koordinatensystem zur Berechnung des Sputteryields nach BH-Theorie. $\mathrm{R}$ \begin{tabular}{|c|}
\hline ist der Radius für die Krümmung im Punkt O. Die Achsen $x$ und $x^{\prime}$ liegen \\
\hline
\end{tabular} in der Einfallsebene [25]. . . . . . . . . . . . . . . . . . . . . . . 9

2.4. Schematische Dartellung des Massentransportes durch Ionenbeschuss. $\theta$ ist der Einfallswinkel bezüglich Oberflächennormale. $\Delta=d_{R}-d_{V}$. . . . . . . 11

2.5. Massentransport führt für Einfallswinkel $0<\theta<45^{\circ}$ zu eine Oberflächen\begin{tabular}{|c|}
\hline stabilität. Für Einfallswinkel $\theta>45^{\circ}$ führt die Veränderung des Massen- \\
\hline transpotes entlang der Richtung $s$ (parallel zur lokalen Oberfläche) zu einer \\
\hline Oberflächeninstabilität.
\end{tabular}

2.6. Beim Eindringen eines energiereichen Ionen in die Oberfläche mit Einfalls-

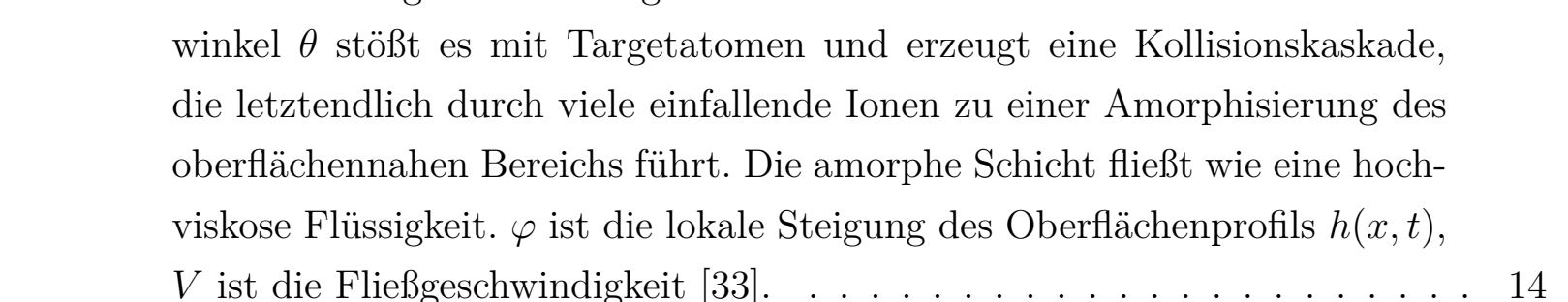

2.7. Analogie zwischen Ionenstrahlsputtern und Flüssigkeitsströmung. Das Io\begin{tabular}{|l|}
\hline nenstrahlsputternsystem kann als eine Flüssigkeit, die eine geneigte Ebene \\
\hline mit Neigungswinkel $\theta$ herunterfließt, gesehen werden. Die externe Volumen- \\
\hline kraft $\vec{b}$ ersetzt hier die Gravitationskraft 32 . . . . . . . . . . . . . . . . . . 15
\end{tabular}

2.8. Die Krümmungskoeffizienten $C_{11}\left(S_{x}\right)$ und $C_{22}\left(S_{y}\right)$ als Funktion der Ein\begin{tabular}{|c|}
\hline fallswinkels: Die experimentellen Werte von $C_{11}$ (schwarze Kreise) und $C_{22}$ \\
\hline \hline (graue Kreise) wie berichtet in [8, 93] für $\mathrm{Ar}^{+} / \mathrm{Si}$ (a) und in [9] für $\mathrm{Kr}^{+} / \mathrm{Si}$ \\
\hline (b). Die rote gestrichelte Linie ist die Voraussage von $C_{11}$ mit Hilfe Gl. \\
\hline (2.38) [127]. . . . . . . . . . . . . . . . . . . . . . . . . . . 20
\end{tabular} 
2.9. Schematische Darstellung eines Ionenstrahls auf eine geneigte Oberfläche 70 .

2.10. (a). Tiefenverteilung der rückgestoßenen Atome berechnet mit dem Simulationsprogramm SDTrimSP. (b). Die mittlere Tiefe der rückgestoßenen Atome als Funktion vom Einfallswinkel. Die gestrichelte Kurve zeigt eine

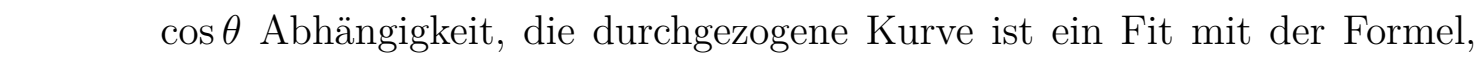

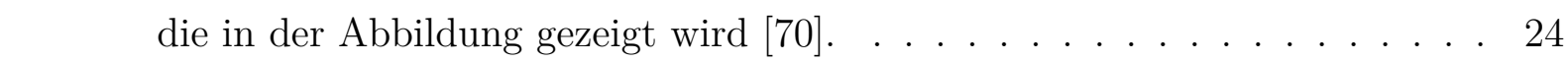

2.11. TEM-Aufname einer Siliziumoberfläche bestrahlt mit $120 \mathrm{keV} \mathrm{Ar}{ }^{+}$bei $60^{\circ}$. Der helle Bereich zeigt die bestrahlte viskose Oberfläche. Der dunkle Bereich zeigt das unbestrahlte Interface [36]. . . . . . . . . . . . . . . . 25

2.12. Skizze einer mit Ionen bestrahlten viskosen Oberflächenschicht. Die Dicke $h_{0}$ ist für eine flache Oberfläche bestrahlt mit Ionen bei einem Einfallswinkel $\theta$. Die obere Kurve ist das Höhenprofil $h$ einer mit Rippel geformten

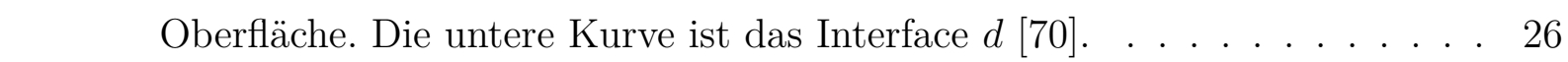

2.13. Driftgeschwindigkeit der rückgestoßenen Atomen als Funktion der Tiefe für

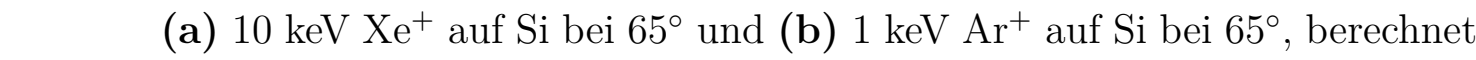
mit SDTrimSP [42]. Das Tiefeninkrement ist $0.1 \mathrm{~nm}$. . . . . . . . . . . . 27

2.14. Skizze eines ionenstrahlinduzierten Massentransports in einer dünnen Fest\begin{tabular}{|c|}
\hline körperschicht mit der Atomdichte $n$ und für eine Fluenz $\Phi \cos \theta=n^{2 / 3}$. \\
\hline \hline$Y / n^{1 / 3}$ ist die mittlere Erosionstiefe, A die Stelle, wo sich der Massen- \\
\hline transport in die gleiche Position zurück entspannt. B ist die Stelle mit \\
\hline einer positiven Steigung und $\mathbf{C}$ mit einer negativen Steigung. Die langen \\
\hline dicken Pfeile zeigen die einfallend Ionen. Die langen dünnen Pfeile zeigen \\
\hline der Massentransport von einer imaginären atomaren Oberflächenschicht \\
\hline \hline verschoben durch die Ionen-Fluenz um $-Y / n^{1 / 3}$. Die kurzen dicken Pfeile \\
\hline \hline geben die Verschiebung der Distanz $Y / n^{1 / 3} \cdot \gamma$ an [70]. . . . . . . . . . . 3 31
\end{tabular}

2.15. (a) Das übliche Koordinatensystem, das in Musterbildungsmodellen ver-

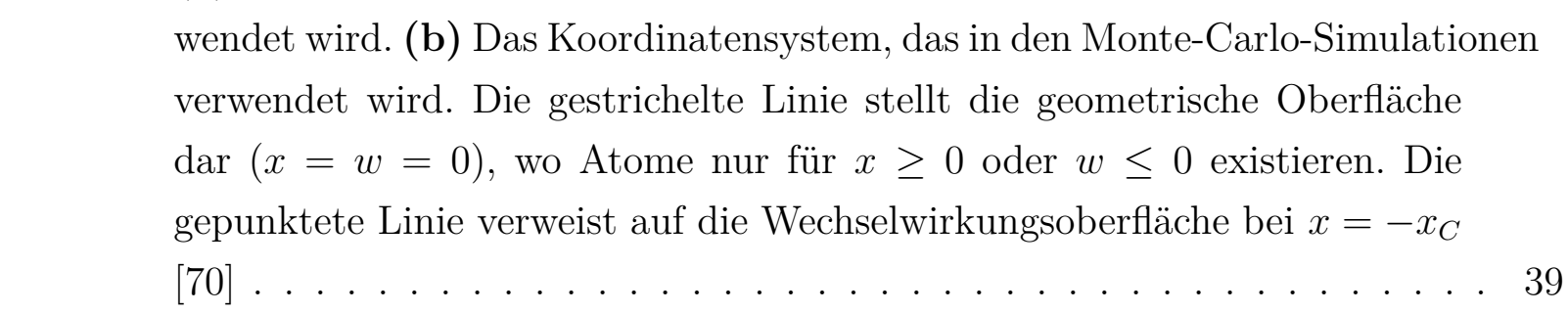

2.16. Zusammenstellung der Krümmungskoeffizienten, die zu $C_{11}$ beitragen, für den Fall $500 \mathrm{eV} \mathrm{Ne}{ }^{+}$auf amorphe Kohlenstoffschicht. . . . . . . . . . . . . 43 
2.17. Schematische Darstellung der Sufactant-Sputtern-Prinzip. Ein homogener \begin{tabular}{|c|}
\hline breiter Ionenstrahl trifft auf das Substrat unter simultaner Kodeposition \\
\hline
\end{tabular} von Surfactant-Atomen. . . . . . . . . . . . . . . . . . . . . . . . . . 45

2.18. Szenario für die Musterbildung auf Si-Oberflächen während der Kodeposition von $\mathrm{Fe}_{-}, \mathrm{Ni}-, \mathrm{Mo}-$, und W-Atome. . . . . . . . . . . . . . . . . . . 46

3.1. Schematischer Aufbau der Plasmakammer. . . . . . . . . . . . . . . . . . . 48

3.2. Unterschiedliche Gas-Plasma erzeugt an der Anlage (Tectra Gen II). Rot für Luft, Violett für Argon und Hellblau für Helium [5] . . . . . . . . . . . . 48

3.3. Schematische Darstellung des Ionenbeschleunigers IOSCHKA. a) Strah\begin{tabular}{|c|}
\hline lungskammer mit Ionenquelle, b) Implantationskammer und c) Vergröße- \\
\hline
\end{tabular} rung der Implantationskammer [60] . . . . . . . . . . . . . . . . . . . . . . 49

3.4. Schema des Ionenbeschleunigers ADONIS [57]. . . . . . . . . . . . . . 50

3.5. Funktionsprinzip eines Rasterkraftmikroskop [4]. . . . . . . . . . . . . . . 51

3.6. Verlauf des Lennard-Jones-Potentials für $c_{1} / c_{2}=10^{-3}$. Die Abbildung zeigt die Summe aus abstoßenden und anziehenden Kräften. [25]. . . . . . . . . . 52

3.7. Schematische Darstellung der Rückstreuung eines Ion in der Tiefe $x$. . . . . 53

3.8. Abhähngigkeit des kinematischen Faktors vom Massenverhältnis $M_{P} / M_{T}$

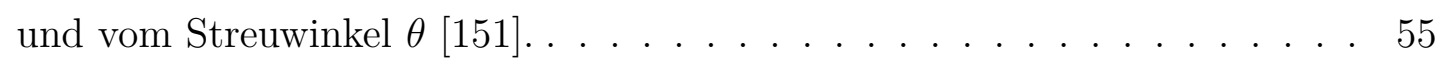

3.9. RBS-Spektrum von $200 \mathrm{~nm}$ dicken Germanium auf Si-Substrat. Die Kreise stellen die experimentellen Daten dar. Die rote Linie ist das simulierte

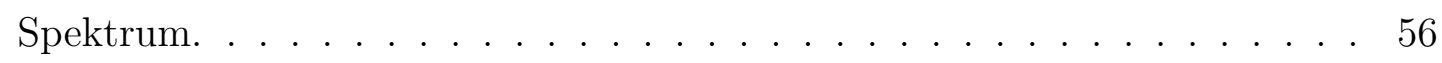

4.1. AFM-Aufnahmen von ta-C Schichten nach der Bestrahlung von $\mathrm{Ne}^{+}$-Ionen. \begin{tabular}{|c|}
\hline Jedes Bild zeigt einen $500 \times 500 \mathrm{~nm}^{2}$ großen Ausschnitt, außer bei 5 und \\
\hline
\end{tabular} $10 \mathrm{keV} 1 \times 1 \mu \mathrm{m}^{2} . \ldots \ldots \ldots \ldots \ldots \ldots$

4.2. Diagramm der gemessenen Wellenlängen für die $\mathrm{Ne}^{+}$-Bestrahlung mit Energien zwischen $250 \mathrm{eV}$ und $10 \mathrm{keV}$ als Funktion der Ionenenergie und des Ioneneinfallswinkels. Der gestrichelte rechteckige Bereich markiert den Bereich, in dem Rippelmuster beobachtet wurden. . . . . . . . . . . . . . . . 59 
4.3. Berechnete Krümmungskoeffizienten $C_{11}^{\text {total }}$ für $250 \mathrm{eV}$ und $500 \mathrm{eV} \mathrm{Ne}{ }^{+}$ Ionenbestrahlung von ta-C. Der Erosionskoeffizient $C_{11}^{\text {eros. }}$ beinhaltet die Krümmungsabhängigkeit der Erosionskraterfunktion $T_{11}$. Im oberen Diagramm beinhaltet $C_{11}^{\text {total }}$ den Effekt der Ionenimplantation $C_{11}^{\text {implant. }}$, aber \begin{tabular}{|l}
\hline die Dickenanhängigkeit der bestrahlten Schicht $D_{11}$ ist vernachlässigt. Im \\
\hline mittleren Diagramm beinhaltet $C_{11}^{\text {total }}$ die Dickenabhängigkeit $D_{11}$, aber
\end{tabular}

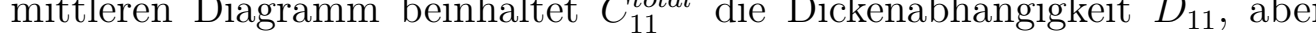
der Effekt der Ionenimplantation $C_{11}^{\text {implant. }}$ ist nicht berücksichtigt. Im unteren Diagramm sind die beiden Koeffizienten $D_{11}$ und $C_{11}^{\text {implant. }}$ berücksichtigt. Der grau schattierte Bereich zeigt die Winkelbereiche an, in denen $C_{11}^{\text {total }}<0$ ist. . . . . . . . . . . . . . . . . . 61

4.4. Berechnete Krümmungskoeffizienten $C_{11}^{\text {total }}$ für $850 \mathrm{eV}$ und $1.5 \mathrm{keV} \mathrm{Ne}{ }^{+}$ Ionenbestrahlung von ta-C. Der Erosionskoeffizient $C_{11}^{\text {eros. }}$ beinhaltet die Krümmungsabhängigkeit der Erosionskraterfunktion $T_{11}$. Im oberen Diagramm beinhaltet $C_{11}^{\text {total }}$ den Effekt der Ionenimplantation $C_{11}^{\text {implant. }}$, aber die Dickenanhängigkeit der bestrahlten Schicht $D_{11}$ ist vernachlässigt. Im mittleren Diagramm beinhaltet $C_{11}^{\text {total }}$ die Dickenabhängigkeit $D_{11}$, aber der Effekt der Ionenimplantation $C_{11}^{\text {implant. }}$ ist nicht berücksichtigt. Im unteren Diagramm sind die beiden Koeffizienten $D_{11}$ und $C_{11}^{\text {implant. }}$ berücksichtigt. Der grau schattierte Bereich zeigt die Winkelbereiche an, in denen $C_{11}^{\text {total }}<0$ ist. . . . . . . . . . . . . . . . 62

4.5. Berechnete Krümmungskoeffizienten $C_{11}^{\text {total }}$ für $5 \mathrm{keV}$ und $10 \mathrm{keV} \mathrm{Ne}{ }^{+}$ Ionenbestrahlung von ta-C. Der Erosionskoeffizient $C_{11}^{\text {eros. }}$ beinhaltet die Krümmungsabhängigkeit der Erosionskraterfunktion $T_{11}$. Im oberen Diagramm beinhaltet $C_{11}^{\text {total }}$ den Effekt der Ionenimplantation $C_{11}^{\text {implant. }}$, aber \begin{tabular}{|l}
\hline die Dickenanhängigkeit der bestrahlten Schicht $D_{11}$ ist vernachlässigt. Im \\
\hline \hline mittleren Diagramm beinhaltet $C_{11}^{\text {total }}$ die Dickenabhängigkeit $D_{11}$, aber
\end{tabular} der Effekt der Ionenimplantation $C_{11}^{\text {implant. }}$ ist nicht berücksichtigt. Im unteren Diagramm sind die beiden Koeffizienten $D_{11}$ und $C_{11}^{\text {implant. }}$ berücksichtigt. Der grau schattierte Bereich zeigt die Winkelbereiche an, in denen

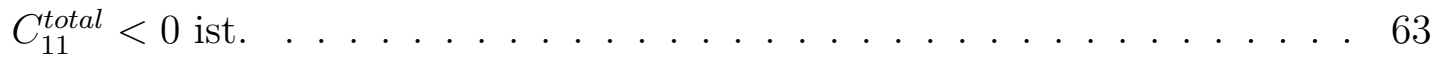

4.6. Berechnete Krümmungskoeffizienten $C_{22}^{\text {total }}$ für $250 \mathrm{eV}$ und $500 \mathrm{eV} \mathrm{Ne}{ }^{+}$ Ionenbestrahlung von ta-C. Der Erosionskoeffizient $C_{22}^{\text {eros. }}$ beinhaltet die Krümmungsabhängigkeit der Erosionskraterfunktion $T_{22}$. Im oberen Diagramm beinhaltet $C_{22}^{\text {total }}$ den Effekt der Ionenimplantation $C_{22}^{\text {implant. }}$. Im unteren Diagramm wird der Effekt der Ionenimplantation nicht berücksichtigt. 64 
4.7. Berechnete Krümmungskoeffizienten $C_{22}^{\text {total }}$ für $850 \mathrm{eV}$ und $1.5 \mathrm{keV} \mathrm{Ne}{ }^{+}$ Ionenbestrahlung von ta-C. Der Erosionskoeffizient $C_{22}^{\text {eros. }}$ beinhaltet die Krümmungsabhängigkeit der Erosionskraterfunktion $T_{22}$. Im oberen Diagramm beinhaltet $C_{22}^{\text {total }}$ den Effekt der Ionenimplantation $C_{22}^{\text {implant. }}$. Im unteren Diagramm wird der Effekt der Ionenimplantation nicht berücksichtigt. 65

4.8. Berechnete Krümmungskoeffizienten $C_{22}^{\text {total }}$ für $5 \mathrm{keV}$ und $10 \mathrm{keV} \mathrm{Ne}{ }^{+}$ Ionenbestrahlung von ta-C. Der Erosionskoeffizient $C_{22}^{\text {eros. }}$ beinhaltet die Krümmungsabhängigkeit der Erosionskraterfunktion $T_{22}$. Im oberen Diagramm beinhaltet $C_{22}^{\text {total }}$ den Effekt der Ionenimplantation $C_{22}^{\text {implant. }}$ Im unteren Diagramm wird der Effekt der Ionenimplantation nicht berücksichtigt. 66

4.9. Wellenlänge der in Abbildung (4.10) gezeigten Rippelmuster als Funktion der Ionenenergie für einen festen Ioneneinfallswinkel von $60^{\circ}$. . . . . . . . 67

4.10. AFM-Bilder von Rippelmustern auf ta-C-Schichten, die mit $\mathrm{Ar}^{+}$-Ionen unter einem Einfallswinkel von $60^{\circ}$ und unterschiedlichen Ionenenergien zwischen $500 \mathrm{eV}$ und $60 \mathrm{keV}$ erodiert wurden. Die Rippel sind mit einem Wellenvektor parallel zur projizierten Ionenstrahlrichtung orientiert. Jedes Bild zeigt einen $1 \times 1 \mu \mathrm{m}^{2}$ großen Ausschnitt. . . . . . . . . . . . . . . . . . . . 68

4.11. AFM-Aufnahmen von den ta-C Schichten nach der Bestrahlung von $\mathrm{Ar}^{+}$-

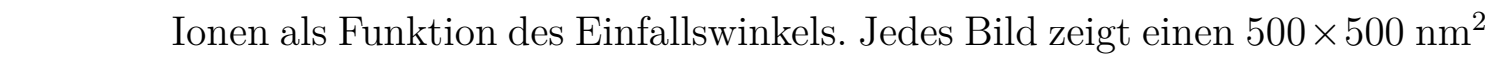
großen Ausschnitt außer bei $10 \mathrm{keV} 1 \times 1 \mu \mathrm{m}^{2}$. . . . . . . . . . . . . . . . . 69

4.12. Berechnete Krümmungskoeffizienten $C_{11}^{\text {total }}$ für $500 \mathrm{eV}$ und $1 \mathrm{keV} \mathrm{Ar}^{+}$Ionenbestrahlung von ta-C. Der Koeffizient $C_{11}^{\text {eros. }}$ beinhaltet die Krümmungsabhängigkeit der Erosionskraterfunktion $T_{11}$. Im oberen Diagramm beinhaltet $C_{11}^{\text {total }}$ den Effekt der Ionenimplantation $C_{11}^{\text {implant. }}$, aber die Dickenabhängigkeit der bestrahlten Schicht $D_{11}$ ist vernachlässigt. Im mittleren Diagramm beinhaltet $C_{11}^{\text {total }}$ die Dickenabhängigkeit $D_{11}$ aber der Effekt der Ionenimplantation $C_{11}^{\text {implant. }}$ ist nicht berücksichtigt. Im unteren Diagramm sind die beiden Koeffizienten $D_{11}$ und $C_{11}^{\text {implant. }}$ berücksichtigt. Der grau schattierte Bereich zeigt die Winkelbereiche an, in denen $C_{11}^{\text {total }}<0$ ist. 70 
4.13. Berechnete Krümmungskoeffizienten $C_{11}^{\text {total }}$ für $10 \mathrm{keV} \mathrm{Ar}^{+}$-Ionenbestrahlung

\begin{tabular}{|c|}
\hline von ta-C. Der Erosionskoeffizient $C_{11}^{\text {eros. }}$ beinhaltet die Krümmungsabhän- \\
\hline gigkeit der Erosionskraterfunktion $T_{11}$. Im oberen Diagramm beinhaltet \\
\hline$C_{11}^{\text {total }}$ den Effekt der Ionenimplantation $C_{11}^{\text {implant. }}$, aber die Dickenabhängig- \\
\hline keit der bestrahlten Schicht $D_{11}$ ist vernachlässigt. Im mittleren Diagramm \\
\hline beinhaltet $C_{11}^{\text {total }}$ die Dickenabhängigkeit $D_{11}$, aber der Effekt der Ionenim- \\
\hline plantation $C_{11}^{\text {implant. }}$ ist nicht berücksichtigt. Im unteren Diagramm sind die \\
\hline beiden Koeffizienten $D_{11}$ und $C_{11}^{\text {implant. }}$ berücksichtigt. Der grau schattierte \\
\hline Bereich zeigt die Winkelbereiche an, in denen $C_{11}^{t o t a l}<0$ ist. . . . . . . . \\
\hline
\end{tabular}

4.14. Berechnete Krümmungskoeffizienten $C_{22}^{\text {total }}$ für $5 \mathrm{keV}$ und $10 \mathrm{keV} \mathrm{Ne}{ }^{+}$\begin{tabular}{|l|}
\hline Ionenbestrahlung von ta-C. Der Erosionskoeffizient $C_{22}^{\text {eros. }}$ beinhaltet die \\
\hline \hline Krümmungsabhängigkeit der Erosionskraterfunktion $T_{22}$. Im oberen Dia- \\
\hline gramm beinhaltet $C_{22}^{\text {total }}$ den Effekt der Ionenimplantation $C_{22}^{\text {implant. }}$. Im un- \\
\hline teren Diagramm wird der Effekt der Ionenimplantation nicht berücksichtigt. 72
\end{tabular}

4.15. Berechnete Krümmungskoeffizienten $C_{22}^{\text {total }}$ für $1 \mathrm{keV}$ und $10 \mathrm{keV} \mathrm{Ar}^{+}$Ionenbestrahlung von ta-C. Der Erosionskoeffizient $C_{22}^{\text {eros. }}$ beinhaltet die Krümmungsabhängigkeit der Erosionskraterfunktion $T_{22}$. Im oberen Diagramm beinhaltet $C_{22}^{\text {total }}$ den Effekt der Ionenimplantation $C_{22}^{\text {implant. }}$. Im unteren Diagramm wird der Effekt der Ionenimplantation nicht berücksichtigt. 73

4.16. AFM-Aufnahmen von den Si-Schichten nach der Bestrahlung von $500 \mathrm{eV}$,

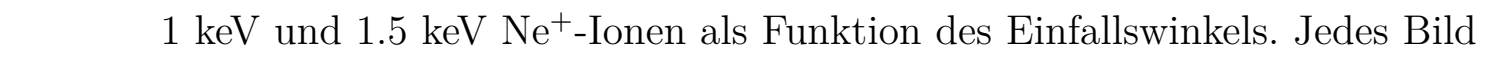
zeigt einen $1 \times 1 \mu \mathrm{m}^{2}$ großen Ausschnitt. . . . . . . . . . . . . . . 76

4.17. Berechnete Krümmungskoeffizienten $C_{11}^{\text {total }}$ für $500 \mathrm{eV}$ und $1 \mathrm{keV} \mathrm{Ne}^{+}$-

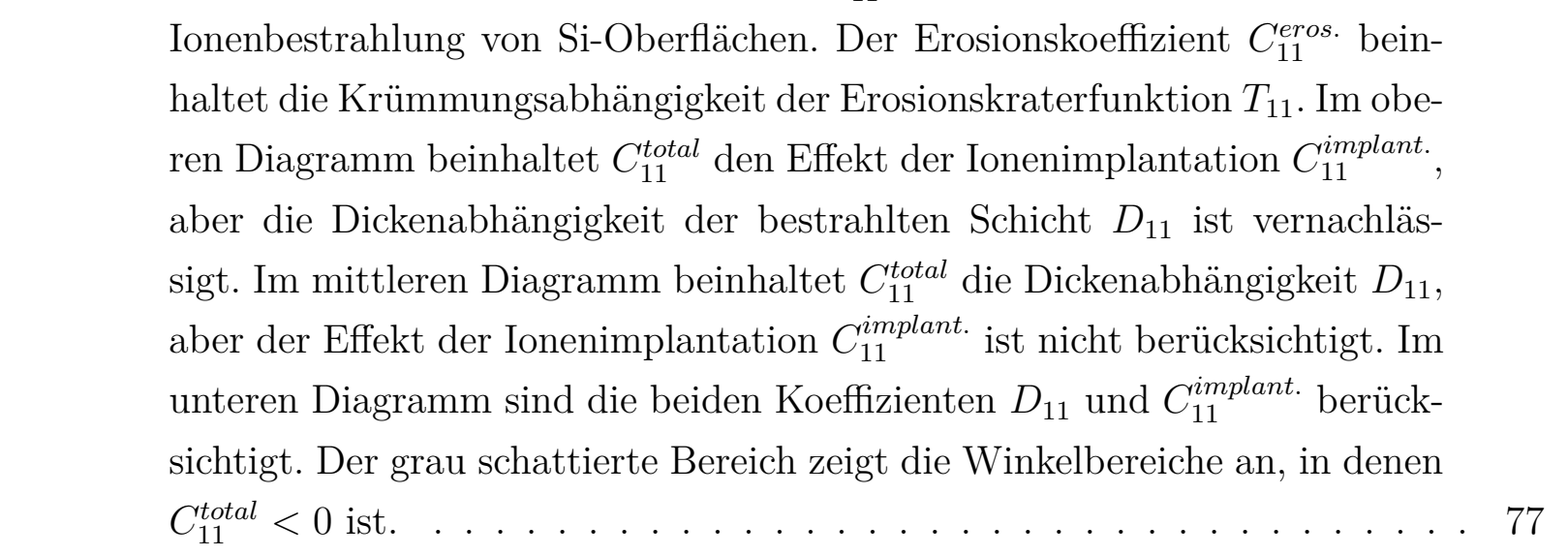


4.18. Berechnete Krümmungskoeffizienten $C_{11}^{\text {total }}$ für $1.5 \mathrm{keV} \mathrm{Ne}{ }^{+}$-Ionenbestrahlung

\begin{tabular}{|c|}
\hline von Si-Oberflächen. Der Erosionskoeffizient $C_{11}^{\text {eros. }}$ beinhaltet die Krüm- \\
\hline mungsabhängigkeit der Erosionskraterfunktion $T_{11}$. Im oberen Diagramm \\
\hline \hline beinhaltet $C_{11}^{\text {total }}$ den Effekt der Ionenimplantation $C_{11}^{\text {implant. }}$, aber die Di- \\
\hline ckenabhängigkeit der bestrahlten Schicht $D_{11}$ ist vernachlässigt. Im mittle- \\
\hline ren Diagramm beinhaltet $C_{11}^{\text {total }}$ die Dickenabhängigkeit $D_{11}$, aber der Effekt \\
\hline \hline der Ionenimplantation $C_{11}^{\text {implant. } \text { ist nicht berücksichtigt. Im unteren Dia- }}$ \\
\hline \hline gramm sind die beiden Koeffizienten $D_{11}$ und $C_{11}^{\text {implant. } \text { berücksichtigt. Der }}$ \\
\hline \hline grau schattierte Bereich zeigt die Winkelbereiche an, in denen $C_{11}^{\text {total }<0 \text { ist. }} 78$
\end{tabular}

4.19. Berechnete Krümmungskoeffizienten $C_{22}^{\text {total }}$ für $500 \mathrm{eV}$ und $1 \mathrm{keV} \mathrm{Ne}{ }_{-}$ Ionenbestrahlung von Si-Oberflächen. Der Erosionskoeffizient $C_{22}^{\text {eros. }}$ beinhaltet die Krümmungsabhängigkeit der Erosionskraterfunktion $T_{22}$. Im obe\begin{tabular}{|c|}
\hline \hline ren Diagramm beinhaltet $C_{22}^{\text {total }}$ den Effekt der Ionenimplantation $C_{22}^{\text {implant. }}$. \\
\hline
\end{tabular} Im unteren Diagramm wird der Effekt der Ionenimplantation nicht berück-

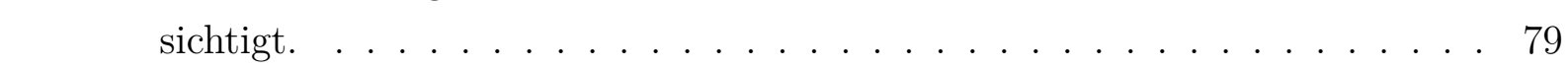

4.20. Berechnete Krümmungskoeffizienten $C_{22}^{\text {total }}$ für $1.5 \mathrm{keV} \mathrm{Ne}{ }^{+}$-Ionenbestrahlung \begin{tabular}{|c|}
\hline von Si-Oberflächen. Der Erosionskoeffizient $C_{22}^{\text {eros. }}$ beinhaltet die Krüm- \\
\hline \hline mungsabhängigkeit der Erosionskraterfunktion $T_{22}$. Im oberen Diagramm \\
\hline beinhaltet $C_{22}^{\text {total }}$ den Effekt der Ionenimplantation $C_{22}^{\text {implant. }}$ Im unteren \\
\hline Diagramm wird der Effekt der Ionenimplantation nicht berücksichtigt. . . . 80
\end{tabular}

4.21. Simulationsergebnisse für $500 \mathrm{eV}$ und $1 \mathrm{keV} \mathrm{Ne} \mathrm{N}^{+}$auf Si. Gezeigt sind die

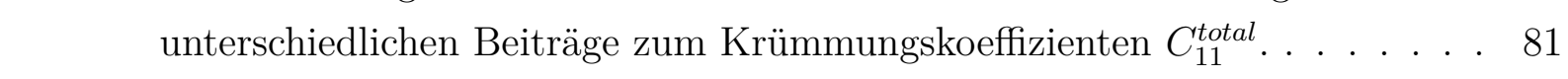

5.1. Phasendiagramme der Systeme C-Ti und C-Mo (Quelle: [2]) . . . . . . . . 84

5.2. $\quad$ Phasendiagramme der Systeme W-Ti und Pt-Mo (Quelle: [2]) . . . . . . . 85

5.3. Schematische Darstellung des Versuchsaufbaus. ta-C-Schicht wird mit 1 $\mathrm{keV} \mathrm{Xe}^{+}$- oder $\mathrm{Ar}^{+}$-Ionen bei normalem Ioneneinfall bestrahlt. MetallKodeposition stammt von benachbarten Metallfolien-Targets, die um $60^{\circ}$ \begin{tabular}{|c|}
\hline geneigt sind. Die Positionen $x_{i}$ zeigen unterschiedliche Analysepositionen \\
\hline
\end{tabular} für AFM- und RBS-Messungen an. . . . . . . . . . . . . . . . 86

5.4. Zusammenstellung von AFM-Bildern von Surfactant-induzierten Mustern \begin{tabular}{|c|}
\hline auf ta-C für verschiedene Metall-Surfactant-Atome (Ti, Mo, W, Pt) und \\
\hline unterschiedliche Metallabdeckung. Die Bedeckung in Atomeinheiten wurde \\
\hline mit RBS gemessen. Die Proben wurden mit $1 \mathrm{keV} \mathrm{Xe}{ }^{+}$-Ionen bestrahlt, mit \\
\hline Ausnahme von Ti-Surfactant, die Probe wurde mit $\mathrm{Ar}^{+}$-Ionen bestrahlt. \\
\hline Die Metall-Kodepostion erfolgte von der linken Seite der gezeigten Bilder. . 88
\end{tabular} 
5.5. Hochauflösende TEM-Analyse des Rippel-Profils. Vor der TEM-Analyse wurde eine amorphe Kohlenstoffschicht auf der Oberfläche deponiert um den Kontrast zwischen der Metall-taC-Schicht und dem Pt-C-Kleber sicherzustellen. Die Ionenstrahlrichtungen sind als Pfeile angezeigt. a) QuerschnittsTEM-Bild einer ta-C-Probe, bestrahlt mit 1 keV Xe ${ }^{+}$-Ionen und Mo-Surfactant. b) Querschnitts-TEM-Bild einer ta-C-Probe, bestrahlt mit $1 \mathrm{keV} \mathrm{Xe}^{+}$Ionen und W-Surfactant. c) Querschnitts-TEM-Bild einer ta-C-Probe, bestrahlt mit $1 \mathrm{keV} \mathrm{Ar}^{+}$-Ionen und Ti-Surfactant. . . . . . . . . . . . . . . . 89

5.6. AFM-topographische Bilder von ta-C-Oberflächen, die mit $1 \mathrm{keV} \mathrm{Ar}^{+}$- und $\mathrm{Xe}^{+}$-Ionen bei normalem Ioneneinfall und einer Fluenz von $10^{18} / \mathrm{cm}^{2}$ erodiert wurden. Die Oberflächen sind extrem glatt mit einer rms Rauigkeit von $0.3 \mathrm{~nm} . \ldots \ldots \ldots$ 


\section{Tabellenverzeichnis}

4.1. Konzentration von implantierten Ne-Ionen und des restlichen Ne-Gehalts, berechnet mit SDTrimSP. Die fünfte Spalte zeigt den Anteil der durch die Ionenbestrahlung wieder gesputterten Ne-Atomen. Die letzte Spalte zeigt den gemessenen restlichen Ne-Gehalt unter Verwendung der RutherfordRückstreu-Spektroskopie. Der Ne-Gehalt ist bezogen auf die Fluenz in der dritten Spalte. . . . . . . . . . . . . . . . . . . . . . . . . . . . . . . . . . . 60 



\section{A. Publikationen}

Teile der in dieser Arbeit vorgestellten Ergebnisse wurden in den folgenden Artikeln veröffentlicht:

- Omar Bobes, Hans Hofsäss, and Kun Zhang. Neon ion beam induced pattern formation on amorphous carbon surfaces. AIP Advances 8, 025205 (2018).

- Omar Bobes, Ronja Langendorf, and Hans Hofsäss. Noble gas ion-induced pattern formation on indium-tin oxide thin films. Thin solid films (submitted).

- Hans Hofsäss, Omar Bobes. Simulation of ion-induced nanopattern formation using Mote Carlo simulations and comparison to experiments (submitted).

- Hans Hofsäss, Kun Zhang, and Omar Bobes. Self-organized surface ripple pattern formation by ion implantation. Journal of Applied Physics, 120(13):135308, 2016.

- Hans Hofsäss, Omar Bobes, and Kun Zhang. Argon ion beam induced surface pattern formation on Si. Journal of Applied Physics, 119(3):035302, 2016.

Weitere wissenschaftliche Veröffentlichungen:

- Kun Zhang, Omar Bobes, and Hans Hofsäss.Designing self-organized nanopatterns on Si by ion irradiation and metal co-deposition. Nanotechnology, 25(8):085301, 2014.

- Hans Hofsäss, Omar Bobes, and Kun Zhang. Is Sputtering Relevant For Ion-Induced Self-Organized Pattern Formation?. AIP Conference Proceedings 1525, 386 (2013).

- Omar Bobes, Kun Zhang, and Hans Hofsäss. Ion beam induced surface patterns due to mass redistribution and curvature-dependent sputtering. Physical Review B, 86(23):235414, 2012.

- H Hofsäss, K Zhang, A Pape, O Bobes, and M Brötzmann. The role of phase separation for self-organized surface pattern formation by ion beam erosion and metal atom co-deposition. Applied Physics A, 111(2):653â664, 2013. 



\section{B. Danksagung}

Hiermit möchte ich mich bei allen bedanken, die mir direkt oder indirekt bei der Erstellung dieser Doktorarbeit geholfen haben. An erste Stelle möchte ich mich herzlich bei Herrn Prof. Dr. Hans Hofsäss für die Vergabe des interessanten Themas meiner Doktorarbeit und seine sehr gute wissenschaftliche Betreuung bedanken. Besonders bedanken möchte ich mich für seine großartige Hilfe bei den Monte-Carlo Simulationen mit SDTrimSPProgramm und die Finanzierung meiner Promotion und meiner Teilnahme am Workshop nano pattern formation at surfaces in Dänemark, am Ionenstrahlworkshop in Heidelberg sowie an der DPG-Tagungen in Berlin, Dresden und Regensburg. Ohne die Unterschtützung von Herrn Prof. Hofsäss wäre ich nie so weit gekommen.

Dr. Kun Zhang danke ich vielmals für seine tolle Betreuung, seine Geduld mit mir und die schönen Zeiten in Dänemark, Berlin, Dresden und Augsburg.

Mein Dank gilt allen Mitarbeitern der Feinwerkstatt, namentlich Klaus Arndt, Peter Arnsberger und Andreas König, für die schnelle Ausführung einer unzählbaren Menge von Aufträgen. Andreas, vor drei Monaten hast Du uns plötzlich und unerwartet verlassen. Leider konnten wir uns nicht richtig verabschieden. Ich wünschte, Du wärst hier, um mit uns meine Promotion zu feiern. Ich danke Dir für deine Hilfe und Unterstützung während meiner Promotion und für die interessanten Diskussionen über Religion und Geschichte. Weiter möchte ich mich bei allen Mitarbeitern des II. physikalischen Institut in Göttingen bedanken. Vor allem bedanke ich mich bei Alexander Rahn für seine Hilfsbereitschaft. Ein weiterer großer Dank gilt Dr. Ulrich Vetter für seine Korrekturlesen und Interesse an meiner Arbeit.

Schließlich möchte ich erwähnen, dass dieser lange harte Weg niemals ohne die Hilfe meiner Eltern zum guten Ende hätte kommen können. Ganz besonders möchte ich mich daher bei meiner Eltern bedanken, die mich in allen Jahren meiner Ausbildung, Studium und Doktorandenzeit immer wieder durch ihre Motivation, finanzielle Unterstützung und Gebet unterstützt haben.

Ohne die Hilfe meiner lieben Frau Marwa Alrefaei wäre dieser Arbeit aber auch nicht zustande gekommen. Ich danke Dir für deine seelische, moralische und geistliche Unterstützung sowie für deine Geduld. 


\section{B. Danksagung}

Ich danke dem lieben Gott von ganzem Herzen für die Bewahrung in allen diesen Jahren und für sein Lenken auf seine Art und Weise, wie es eben kein Mensch tun kann. 